\title{
An Overview of Complex Fractal Dimensions: From Fractal Strings to Fractal Drums, and Back
}

\author{
Michel L. Lapidus
}

\begin{abstract}
Our main goal in this long survey article is to provide an overview of the theory of complex fractal dimensions and of the associated geometric or fractal zeta functions, first in the case of fractal strings (one-dimensional drums with fractal boundary), in $\$ 2$ and then in the higher-dimensional case of relative fractal drums and, in particular, of arbitrary bounded subsets of Euclidean space of $\mathbb{R}^{N}$, for any integer $N \geq 1$, in $₫ 3$

Special attention is paid to discussing a variety of examples illustrating the general theory rather than to providing complete statements of the results and their proofs, for which we refer to the author's previous (joint) books mentioned in the paper.

Finally, in an epilogue (44), entitled "From quantized number theory to fractal cohomology", we briefly survey aspects of related work (motivated in part by the theory of complex fractal dimensions) of the author with H. Herichi (in the real case) HerLap1, along with Lap8, and with T. Cobler (in the complex case) CobLap1, respectively, as well as in the latter part of a book in preparation by the author, Lap10.
\end{abstract}

To my daughter, Julie Anne Myriam Lapidus, who has recently embarked on a journey fraught with perilous and frightening dangers, the ultimate struggle for the continuation of her own beautiful and young life.

You are my scintillating treasure and will always remain my untarnished jewel.

With all my love.

2010 AMS Subject Classification:

Primary - 11M06, 11M41, 28A12, 28A80, 30D10, 30B50, 35P20, 42B20, 45Q05, 81Q20.

Secondary - 30D30, 37C30, 37C45, 40A10, 42B35, 42B37, 44A10, 45Q05, 81R40.

Key words and phrases:

Fractal strings and drums, relative fractal drums (RFDs), complex dimensions, fractal zeta functions, distance and tube zeta functions, relative fractal zeta functions, fractal tube formulas, Minkowski dimension and content, Minkowski measurability criteria, fractality, unreality, Riemann zeta function, Riemann hypothesis, direct and inverse problems for

Work partially supported by the US National Science Foundation under the research grants DMS-0707524 and DMS-1107750, as well as by the Burton Jones Endowed Chair Fund while he has been the holder of the F. Burton Jones Endowed Chair in Pure Mathematics at the University of California, Riverside, since July 2017. 
fractal strings, quantized number theory, infinitesimal shift, spectral operator, regularized determinants, fractal cohomology.

\section{Contents}

1. Introduction

2. Fractal Strings and Their Complex Dimensions

2.1. Fractal tube formulas

2.2. Other examples of fractal explicit formulas

2.3. Analogy with Riemann's explicit formula

2.4. The meaning of complex dimensions

2.5. Fractality, complex dimensions and irreality

2.6. Inverse spectral problems and the Riemann hypothesis $\quad 22$

2.6.1. Direct spectral problems for fractal strings $\quad 23$

2.6.2. Inverse spectral problems for fractal strings 24

3. A Taste of the Higher-Dimensional Theory: Complex Dimensions and Relative Fractal Drums (RFDs)

3.1. Brief history

3.2. Fractal zeta functions and relative fractal drums (FZFs and RFDs)

3.2.1. The special case of bounded sets in $\mathbb{R}^{N}$

3.2.2. The special case of fractal strings

3.3. A few key properties of fractal zeta functions

3.3.1. Abscissa of convergence and holomorphicity

3.3.2. Meromorphic continuation and Minkowski content

3.3.3. Scaling property and invariance under isometries

3.3.4. Invariance of the complex dimensions under embedding into higherdimensional spaces

3.4. Examples of fractal zeta functions and complex dimensions 43

3.4.1. The Sierpinski gasket 43

3.4.2. The Sierpinski carpet 44

3.4.3. The 3- $d$ Sierpinski carpet 45

3.4.4. The $N$-dimensional relative Sierpinski gasket $\quad 46$

3.4.5. The $\frac{1}{2}$-square and $\frac{1}{3}$-square fractals 49

3.4.6. The $(N-1)$-sphere and its associated RFD 52

3.4.7. The Cantor grill 54

3.4.8. The Cantor dust 5.5

3.4.9. The devil's staircase (or Cantor graph) RFD $\quad 59$

3.4.10. Self-similar sprays 61

3.5. Fractal tube formulas and Minkowski measurability criteria: Theory and examples

3.5.1. Fractal tube formulas for RFDs in $\mathbb{R}^{N}$, via distance and tube zeta functions

\begin{tabular}{lll} 
3.5.2. Minkowski measurability criteria for RFDs in $\mathbb{R}^{N}$ & 69 \\
\hline
\end{tabular}

3.5.3. Examples $\quad 70$

3.6. Fractality, hyperfractality and unreality, revisited 80

3.6.1. The $\frac{1}{2}$-square fractal, the $\frac{1}{3}$-square fractal and the relative Sierpinski $N$-gasket

3.6.2. The devil's staircase and fractality 
3.6.3. Extended notion of complex dimensions, scaling laws and Riemann surfaces

3.6.4. Maximal hyperfractals and meromorphic extensions of spectral zeta functions of fractal drums

4. Epilogue: From Complex Fractal Dimensions to Quantized Number Theory and Fractal Cohomology

4.1. Analogy between self-similar geometries and varieties over finite fields 89

4.2. Quantized number theory: The real case 91

4.3. Quantized number theory: The complex case 94

4.4. Towards a fractal cohomology 97

4.4.1. Grading of the fractal cohomology by the real parts 99

4.4.2. Open problems and perspectives: Geometric interpretation and beyond 102

Acknowledgements 104

Glossary 105

References 108

\section{Introduction}

Our main goal in this research expository article is to provide an overview of the theory of complex dimensions and of the associated geometric or fractal zeta functions, first in the case of fractal strings (one-dimensional drums with fractal boundary) or essentially equivalently, for compact subsets of the real line (see $\$ 2$ ), and then (see 93 ), in the higher-dimensional case of relative fractal drums and, in particular, of bounded subsets in Euclidean space $\mathbb{R}^{N}$, for any integer $N \geq 1$.

Special attention is paid to providing a variety of examples (especially, in higher dimensions) illustrating the general theory rather than to stating precise theorems in their greatest generality or providing their proofs (or even a sketch thereof). For a rigorous and quite detailed account of the theory, we refer instead the interested reader to the books by the author and Machiel van Frankenhuijsen, Lap-vF4 for the case of fractal strings (i.e., $N=1$ ) and the recent book by the author, Goran Radunović and Darko Žubrinić, LapRaZu1, when $N \geq 1$ is arbitrary.

In the epilogue (see \$4), we survey related work, motivated in part by the theory of complex dimensions of fractal strings, on quantized number theory (in the real setting) and the Riemann hypothesis (joint with Hafedh Herichi; see the papers HerLap2-5 and the forthcoming book HerLap1, along with Lap8), as well as on quantized number theory (in the complex setting), regularized determinants and "fractal cohomology" (see CobLap1-2, joint with Tim Cobler, along with the author's book in preparation, Lap10, of which the present paper is both the seed and a significantly condensed version).

Except at the very end of this introduction, we now focus on $\$ 2$ and $\$ 3$ in the rest of $\$ 1$ For a brief overview of the contents of 4 , we refer the interested reader to the overall introduction of 4 and to the beginning of each of the subsections of \$4 (namely, $\S \$ 4.14 .4$ ), along with part (iii) towards the end of this section.

Complex dimensions provide a natural way to extract the information about the oscillatory nature of fractal objects. This is done via generalized explicit formulas 
(extending to this setting Riemann's original explicit formula for the prime number counting function, see 2.31 .

In this paper, we have chosen to emphasize a type of explicit formulas called fractal tube formulas and which enable us to express the volume, $V(\varepsilon)$, of the $\varepsilon$-neighborhoods of the given fractal set $A$ or, more generally, of the given relative fractal drum $(A, \Omega)\left(\mathrm{RFD}\right.$, in short) in $\mathbb{R}^{N}[1$ as an extended power series in $\varepsilon$ with exponents the underlying complex codimensions and (normalized) coefficients (in the case of simple poles) the residues of the associated geometric or fractal zeta function.

For example, under suitable assumptions (and still in the case of simple poles), we have the following (pointwise or distributional) exact fractal tube formula for a given bounded set $A$ (or, more generally, $\operatorname{RFD}(A, \Omega)$ ) in $\mathbb{R}^{N}$ (up to a possible error term, which can be estimated explicitly):

$$
V(\varepsilon)=\sum_{\omega \in \mathcal{D}} c_{\omega} \frac{\varepsilon^{N-\omega}}{N-\omega}
$$

where $c_{\omega}:=\operatorname{res}\left(\zeta_{A}, \omega\right)$ (or, more generally, $c_{\omega}:=\operatorname{res}\left(\zeta_{A, \Omega}, \omega\right)$ ) for each $\omega \in \mathcal{D}$, and where $\mathcal{D}=\mathcal{D}_{A}$ (respectively, $\mathcal{D}=\mathcal{D}_{A, \Omega}$ ) denotes the set of complex dimensions of $A$ (respectively, of the $\operatorname{RFD}(A, \Omega)$ ), viewed as a multiset (i.e., a set with finite integer multiplicities). Here, $\zeta_{A}$ (respectively, $\left.\zeta_{A, \Omega}\right)$ is the distance zeta function of $A$ (respectively, of $(A, \Omega)$ ) defined (in the case of the bounded set $A$, for example) for all $s \in \mathbb{C}$ with $\operatorname{Re}(s)$ sufficiently large by the Lebesgue (and hence, absolutely convergent) integral

$$
\zeta_{A}(s)=\int_{A_{\delta}} d(x, A)^{s-N} d x
$$

for some fixed $\delta>0$ whose specific value is unimportant from the point of view of the theory of complex dimensions.

More specifically, (1.3) holds for all $s \in \mathbb{C}$ with $\operatorname{Re}(s)>\bar{D}$, where $\bar{D}$ is the (upper) Minkowski dimension of $A$, and this lower bound is optimal. In other words, the abscissa of convergence of $\zeta_{A}$ coincides with $\bar{D}$; this is one of the first basic results of the theory (see part $(a)$ of $\$ 3.3 .1$ ).

The (visible) complex dimensions of $A$ are defined as the poles of the meromorphic continuation (if it exists) of $\zeta_{A}$ to some given connected open neighborhood of the vertical line $\{\operatorname{Re}(s)=\bar{D}\}$ (or, equivalently, of the closed right half-plane $\{\operatorname{Re}(s) \geq \bar{D}\}$, since $\zeta_{A}$ is holomorphic on the open right half-plane $\{\operatorname{Re}(s)>\bar{D}\}$; see part $(b)$ of 33.3 .1$)$

\footnotetext{
${ }^{1}$ An $\operatorname{RFD}$ in $\mathbb{R}^{N}$ is a pair $(A, \Omega)$, with $A \subseteq \mathbb{R}^{N}, \Omega$ open in $\mathbb{R}^{N}$ and $\Omega \subseteq A_{\delta_{1}}$, for some $\delta_{1}>0$, where for any $\varepsilon>0$,

$$
A_{\varepsilon}:=\left\{x \in \mathbb{R}^{N}: d(x, A)<\varepsilon\right\}
$$

is the $\varepsilon$-neighborhood of $A$ and $d(x, A)$ denotes the Euclidean distance from $x \in \mathbb{R}^{N}$ to $A$. Also, we let $V(\varepsilon)=V_{A}(\varepsilon):=\left|A_{\varepsilon}\right|$ (respectively, $V(\varepsilon)=V_{A, \Omega}(\varepsilon):=\left|A_{\varepsilon} \cap \Omega\right|$ ) in the case of a bounded set $A$ (respectively, $\operatorname{RFD}(A, \Omega))$ in $\mathbb{R}^{N}$. Here and thereafter, $|\cdot|=|\cdot|_{N}$ denotes the $N$-dimensional volume (or Lebesgue measure in $\mathbb{R}^{N}$ ).

${ }^{2}$ Throughout this paper, we use the following short-hand notation: given $\alpha \in \mathbb{R}$, we let

$$
\{\operatorname{Re}(s) \geq \alpha\}:=\{s \in \mathbb{C}: \operatorname{Re}(s) \geq \alpha\}
$$

denote the closed right half-plane with abscissa $\alpha$; and analogously for the vertical line $\{\operatorname{Re}(s)=\alpha\}$ or the open right half-plane $\{\operatorname{Re}(s)>\alpha\}$ with abscissa $\alpha$, say. (If $\alpha= \pm \infty$, we adopt the obvious conventions $\{\operatorname{Re}(s) \geq+\infty\}=\emptyset$ and $\{\operatorname{Re}(s) \geq-\infty\}=\mathbb{C}$, for example.)
} 
In particular, if $\bar{D}$ itself is a pole of $\zeta_{A}$ (under mild conditions, it is always a nonremovable singularity of $\zeta_{A}$ ), then it is a complex dimension having the largest possible real part.

Provided $\bar{D}<N$ (i.e., if $\bar{D} \neq N$ since we always have $\bar{D} \leq N$ ), all of the above results and definitions extend to another useful fractal zeta function, called the tube zeta function of $A$ and denoted by $\widetilde{\zeta}_{A}{ }^{3}$ The fractal zeta functions $\zeta_{A}$ and $\widetilde{\zeta}_{A}$ are connected via a functional equation (see (3.15)), which implies that the (visible) complex dimensions of $A$ can be defined indifferently via either $\zeta_{A}$ or $\widetilde{\zeta}_{A}$. Furthermore, the fractal tube formula (1.2) has a simple counterpart expressed in terms of the residues of $\widetilde{\zeta}_{A}$ (instead of those of $\zeta_{A}$ ) evaluated at the complex dimensions of $A$. Namely, up to a possible error term which can be estimated explicitly,

$$
V(\varepsilon)=\sum_{\omega \in \mathcal{D}} d_{\omega} \varepsilon^{N-\omega},
$$

where $d_{\omega}:=\operatorname{res}\left(\widetilde{\zeta}_{A}, \omega\right)$ for each $\omega \in \mathcal{D}$.

All of the above results (including the fractal tube formulas (1.2) and (1.6)) extend to relative fractal drums (RFDs) in $\mathbb{R}^{N}$ (with $\zeta_{A}, \widetilde{\zeta}_{A}$ and $\mathcal{D}=\mathcal{D}_{A}$ replaced by $\zeta_{A, \Omega}, \widetilde{\zeta}_{A, \Omega}$ and $\mathcal{D}=\mathcal{D}_{A, \Omega}$, respectively), which are very useful tools in their own right and enable us, in particular, to compute (by using appropriate decompositions and symmetry considerations) the fractal zeta functions and complex dimensions of many fractal compact subsets of $\mathbb{R}^{N}$.

At this stage, it is helpful to point out that many key results of the theory of complex dimensions of fractal strings Lap-vF4 (briefly discussed in \$2), including the fractal tube formulas (of which (2.10) is a typical example), can be recovered by specializing the higher-dimensional theory of complex dimensions of RFDs in $\mathbb{R}^{N}$ to the $N=1$ case and by viewing fractal strings as RFDs in $\mathbb{R}$. In the process, a simple functional equation connecting the so-called geometric zeta function of a fractal string (described in the beginning of 92 ) and the distance zeta function of the associated RFD plays a key role; see $\$ 3.2 .2$ and $\$ 3.5 .1$

Intuitively, a fractal, viewed as a geometric object, is like a musical instrument tuned to play certain notes with frequencies (respectively, amplitudes) essentially equal to the real parts (respectively, the imaginary parts) of the underlying complex dimensions. Alternatively, one can think of a "geometric wave" propagating through the fractal and with the aforementioned frequencies and amplitudes. This "physical" intuition is corroborated, for example, by the fractal tube formulas expressed via the distance (respectively, tube) zeta function, as in (1.2) (respectively, (1.6) ).

As was mentioned just above, the theory of complex dimensions of fractal strings can be viewed essentially as the one-dimensional special case of the general theory of complex dimensions (of RFDs in $\mathbb{R}^{N}$ ) developed in LapRaZu1. Conversely, fractal string theory has provided the author and his collaborators with a

\footnotetext{
${ }^{3}$ For $\operatorname{Re}(s)$ sufficiently large (in fact, precisely for $\operatorname{Re}(s)>\bar{D}$, provided $\bar{D}<N$ ), $\widetilde{\zeta}_{A}$ is given by the Lebesgue (and hence, absolutely convergent) integral

$$
\widetilde{\zeta}_{A}(s):=\int_{0}^{\delta} V(\varepsilon) \varepsilon^{s-N} \frac{d \varepsilon}{\varepsilon},
$$

for some arbitrary but fixed $\delta>0$, the value of which is unimportant from the point of view of the definition (and the values) of the complex dimensions.
} 
broad and rich collection of examples with which to test various conjectures and formulate various definitions as well as elaborate tools that could eventually be used in more complicated higher-dimensional situations. Also, several of the key steps towards the proof of the higher-dimensional fractal tube formulas (such as in (1.2) and (1.6)) rely, in part, on techniques developed for dealing with the case of (generalized) fractal strings Lap-vF2, Lap-vF3, Lap-vF4.

In addition, "fractality" is characterized (or rather, defined) in our general theory by the presence of nonreal complex dimensions 4 This extends to any dimension $N \geq 1$ the definition of fractality given earlier in [Lap-vF2-4, thanks to the fact that we now have to our disposal a general definition of fractal zeta functions valid for arbitrary bounded (or, equivalently, compact) subsets of $\mathbb{R}^{N}$ (as well as, more generally, for all RFDs in $\left.\mathbb{R}^{N}\right)$.

We will also discuss (in 3.5 .2 when $N \geq 1$ is arbitrary, and in Theorem 2.2 when $N=1$ ) a general Minkowski measurability criterion expressed in terms of complex dimensions. Namely, under certain mild conditions (which imply that the Minkowski dimension $D$ exists and is a complex dimension), a bounded set $A$ (or, more generally, an $\operatorname{RFD}(A, \Omega)$ ) in $\mathbb{R}^{N}$ is Minkowski measurable 5 if and only if its only complex dimension with real part $D$ (i.e., its only principal complex dimension) is $D$ itself and $D$ is simple. In other words, the existence of nonreal complex dimensions (i.e., the "critical fractality" of $A$ or of $(A, \Omega)$; see $\$ 3.6)$, along with the simplicity of $D$ (as a pole of $\zeta_{A}$ or equivalently, of $\zeta_{A, \Omega}$ ), characterizes the Minkowski nonmeasurability of $A$ (or of $(A, \Omega)$ ). As a simple illustration, the Cantor set, the Cantor string, the Sierpinski gasket and the Sierpinski carpet, along with lattice self-similar strings (and more generally, in higher dimensions, lattice self-similar sprays with "sufficiently nice" generators), are all Minkowski nonmeasurable but are Minkowski nondegenerate; see 3.5 .3 On the other hand, a "generic" (i.e., nonlattice) self-similar Cantor-type set (or string) or a "generic" self-similar carpet is Minkowski measurable (because it does not have any nonreal complex dimensions other than $D$, which is simple).

Beside this introduction (i.e., 乌1), this paper is divided into three parts:

( $i)$ 92 a brief account of the theory of complex dimensions for fractal strings $(N=1)$ Lap-vF4 and its prehistory, including a discussion (in 2.6 ) of natural direct and inverse spectral problems for fractal strings along with their intimate connections with the Riemann zeta function LapPo2 and the Riemann hypothesis LapMa2.

(ii) 33, an introduction to the higher-dimensional theory of complex dimensions and the associated fractal zeta functions (namely, the distance and tube zeta functions), based on LapRaZu1 (and aspects of [LapRaZu2-9]), with emphasis on several key examples of bounded sets and relative fractal drums in $\mathbb{R}^{N}$ (with $N=2, N=3$ or $N \geq 1$ arbitrary) illustrating the key concepts of fractal zeta

\footnotetext{
${ }^{4}$ It is also very useful to extend the notion of "complex dimensions" by allowing more general (nonremovable) singularities than poles of the associated fractal zeta functions; see LapRaZu1 and LapRaZu6-7, 10, along with 2.5 3.5.2 3.6 and 4.4

"Intuitively, Minkowski measurability is some kind of "fractal regularity" of the underlying geometry; for a precise definition, see 3.2 when $N \geq 1$ is arbitrary (or 2.1 when $N=1$ ).
} 
functions and their poles or, more generally, nonremovable singularities (i.e., the complex dimensions), as well as the associated fractal tube formulas. As was alluded to earlier, the latter explicit formulas provide a concrete justification of the use of the phrase "complex fractal dimensions" and help explain why both intuitively and in actuality, the theory of complex dimensions is a theory of oscillations that are intrinsic to fractal geometries.

It is noteworthy that even though we will mostly stress the aforementioned geometric oscillations in 93 (and in much of 92 ), the broad definition of "fractality" proposed in $\$ 3.6$ and expressed in terms of the presence of nonreal complex dimensions encompasses oscillations that are intrinsic to number theories (via Riemanntype explicit formulas expressed in terms of the poles and the zeros of attached $L$-functions, or equivalently, in terms of the poles of the logarithmic derivatives of those $L$-functions; see 2.3 for the original example), or to dynamical systems (e.g., via explicit formulas for the counting functions of primitive periodic orbits; see [Lap-vF4, Ch. 7] for a class of examples), as well as to the spectra of fractal drums [both "drums with fractal boundary" (as, e.g., in Lap1-3 and parts of [Lap-vF4) and "drums with fractal membrane" (as, e.g., in Lap3, KiLap1 and Lap7 ) and other classical or quantum physical systems (via detailed spectral asymptotics or, essentially equivalently, via explicit formulas for the associated frequency or eigenvalue counting functions).

Much remains to be done in all of these directions for a variety of specific classes of dynamical systems and of fractal drums, for example. We point out, however, that the deep analogy between many aspects of fractal geometry and number theory (see, e.g., Lap-vF1-5, Lap7, HerLap1 and LapRaZu1) was a key motivation for the author to want to develop (since the mid-1990s) a theory of "fractal cohomology", itself an important motivation for many aspects of the work described in 4 .

(iii) \$4 the epilogue, a very brief account (compared to the size of the corresponding material to be described) of "quantized number theory", both in the "real case" (\$4.2, based on HerLap1, [HerLap2-5 and [Lap8]) and in the "complex case" (\$4.3 based principally on CobLap1-2 and on aspects of Lap10) and the associated fractal cohomology (4.1 and, especially, 4.4. as expanded upon in Lap10 ), with applications to several reformulations of the Riemann hypothesis (4.2) expressed in terms of the "quasi-invertibility" [HerLap1] or the invertibility Lap8 of so-called "spectral operators", in particular, as well as to the representations (\$4.3) of various arithmetic (or number-theoretic) $L$-functions and other meromorphic functions (including the completed Riemann zeta function and the Weil zeta functions attached to varieties over finite fields Wei1-6, Gro1-4, Den1-6, and, e.g., Mani], Kah, Tha1-2] via (graded or supersymmetric) regularized (typically infinite dimensional) determinants of suitable unbounded linear operators (the so-called "generalized Polya-Hilbert operators") restricted to their eigenspaces (which are the proposed "fractal cohomology spaces").

These developments open-up a vast and very rich new domain of research, extending in a variety of directions and located at the intersection of many fields of mathematics, including fractal geometry, number theory and arithmetic geometry, mathematical physics, dynamical systems, harmonic analysis and spectral theory, 
complex analysis and geometry, geometric measure theory, as well as algebraic geometry and topology, to name a few.

We hope that the reader will be stimulated by the reading of this expository article (and eventually, of its much expanded sequel, the author's book in preparation [Lap10]) to explore the various ramifications and consequences of the theory, many of which are yet to be discovered. In other words, instead of offering here a complete and closed theory, we prefer to (and, in fact, must) offer here (especially, in (44) only glimpses of a possible future unifying and "universal" theory, resting on the contributions and conjectures or dreams of many past and contemporary mathematicians and physicists.

\section{Fractal Strings and Their Complex Dimensions}

A (bounded) fractal string can be viewed either as a bounded open set $\Omega \subseteq \mathbb{R}$ or else as a nonincreasing sequence of lengths (or positive numbers) $\mathcal{L}=\left(\ell_{j}\right)_{j=1}^{\infty}$ such that $\ell_{j} \downarrow 0$. (The latter condition is not needed if the sequence $\left(\ell_{j}\right)$ is finite.)

Let us briefly explain the connection between these two points of view. If $\Omega$ is a bounded open subset of $\mathbb{R}$, we can write $\Omega=\cup_{j \geq 1} I_{j}$ as an at most countable disjoint union of bounded open intervals $I_{j}$, of length $\ell_{j}>0$. These intervals are nothing but the connected components of $\Omega$. Since $|\Omega|_{1}=\sum_{j \geq 1} \ell_{j}<\infty$ (i.e., $\Omega$ has finite total length), without loss of generality, we may assume (possibly after having reshuffled the intervals $I_{j}$, that $\ell_{1} \geq \ell_{2} \geq \cdots$ (counting multiplicities) and (provided the sequence $\left(\ell_{j}\right)_{j \geq 1}$ is infinite) $\ell_{j} \downarrow 0$.

Slightly more generally, in the definition of a bounded fractal string, one can assume that instead of being bounded, the open set $\Omega \subseteq \mathbb{R}$ has finite volume (i.e., length): $|\Omega|_{1}<\infty$.

Unbounded fractal strings (i.e., strings $\mathcal{L}=\left(\ell_{j}\right)_{j \geq 1}$ such that $\sum_{j \geq 1} \ell_{j}=+\infty$ ) also play an important role in the theory (see, e.g., [Lap-vF4, Ch. 3 and parts of Chs. 9-11 along with §13.1]) but from now on, unless explicitly mentioned otherwise, we will assume that all of the (geometric) fractal strings under consideration are bounded. As a result, we will often drop the adjective "bounded" when referring to fractal strings.

From a physical point of view, the 'lengths' $\ell_{j}$ can also be thought of as being the underlying scales of the system. This is especially useful in the case of unbounded fractal strings but should also be kept in mind in the geometric situation of fractal strings.

A geometric realization of a (bounded) fractal string $\mathcal{L}=\left(\ell_{j}\right)_{j \geq 1}$ is any bounded open set $\Omega$ in $\mathbb{R}$ (or, more generally, any open set $\Omega$ in $\mathbb{R}$ of finite length) with associated length sequence $\mathcal{L}$.

The geometric zeta function $\zeta_{\mathcal{L}}$ of a fractal string $\mathcal{L}=\left(\ell_{j}\right)_{j \geq 1}$ is defined by

$$
\zeta_{\mathcal{L}}(s)=\sum_{j \geq 1} \ell_{j}^{s}
$$

for all $s \in \mathbb{C}$ with $\operatorname{Re}(s)$ sufficiently large. (Here and henceforth, we let $\ell_{j}^{s}:=\left(\ell_{j}\right)^{s}$, for each $j \geq 1$.)

A simple example of a fractal string is the Cantor string, denoted by $\Omega_{C S}$ (or $\mathcal{L}_{C S}$ ) and defined by $\Omega_{C S}=[0,1] \backslash C$, the complement of the (classic ternary) Cantor set $C$ in the unit interval. (Observe that the boundary of the Cantor string is the Cantor set itself: $\partial \mathcal{L}_{C S}:=\partial \Omega_{C S}=C$.) Then, $\Omega_{C S}$ consists of the disjoint 
union of the deleted (open) intervals, in the usual construction of the Cantor set:

$$
\Omega_{C S}=(0,1 / 3) \cup(1 / 9,2 / 9) \cup(7 / 9,8 / 9) \cup \cdots .
$$

Hence, the associated sequence of lengths $\mathcal{L}_{C S}$ is given by

$$
1 / 3,1 / 9,1 / 9,1 / 27,1 / 27,1 / 27,1 / 27, \cdots ;
$$

equivalently, $\mathcal{L}_{C S}$ consists of the lengths $1 / 3^{n}$ counted with multiplicity $2^{n-1}$, for $n=1,2,3, \cdots$. It follows that $\zeta_{C S}$ can be computed by simply evaluating the following geometric series:

$$
\begin{aligned}
\zeta_{C S}(s) & =\sum_{n=1}^{\infty} 2^{n-1}\left(3^{-n}\right)^{s}=3^{-s} \sum_{n=0}^{\infty}\left(2 \cdot 3^{-s}\right)^{n} \\
& =\frac{3^{-s}}{1-2 \cdot 3^{-s}}=\frac{1}{3^{s}-2}
\end{aligned}
$$

This calculation is valid for $R e(s)>\log _{3} 2$ (i.e., $\left|2 \cdot 3^{-s}\right|<1$ ) but upon analytic continuation, we see that $\zeta_{C S}$ admits a (necessarily unique) meromorphic continuation to all of $\mathbb{C}$ (still denoted by $\zeta_{C S}$, as usual) and that

$$
\zeta_{C S}(s)=\frac{1}{3^{s}-2}, \text { for all } s \in \mathbb{C} \text {. }
$$

The complex dimensions of the Cantor string are the poles of $\zeta_{C S}$; that is, here, the complex solutions of the equation $3^{s}-2=0$. Thus, the set $\mathcal{D}_{C S}$ of complex dimensions of $\mathcal{L}_{C S}$ is given by a single (discrete) vertical line,

$$
\mathcal{D}_{C S}=\{D+i n \mathbf{p}: n \in \mathbb{Z}\}
$$

where $D:=D_{C S}=\log _{3} 2$ is the Minkowski dimension of the Cantor string (or of the Cantor set) and $\mathbf{p}:=2 \pi / \log 3$ is its oscillatory period. (The definition of $D$ is recalled in (2.7). 6

For an arbitrary fractal string $\mathcal{L}$, the complex dimensions of $\mathcal{L}$ (relative to a given domain $U \subseteq \mathbb{C}$ to which $\zeta_{\mathcal{L}}$ admits a meromorphic continuation), also called the visible complex dimensions of $\mathcal{L}$, are simply the poles of $\zeta_{\mathcal{L}}$ which lie in $U$. Thus, for the Cantor string, $\mathcal{D}_{C S}=\mathcal{D}_{C S}(\mathbb{C})$ is given by $(2.5)$.

Recall that the abscissa of convergence $\alpha=\alpha_{\mathcal{L}}$ of the Dirichlet series defining $\zeta_{\mathcal{L}}$ in (2.1) is given by

$$
\alpha:=\inf \left\{\beta \in \mathbb{R}: \sum_{j \geq 1} \ell_{j}^{\beta}<\infty\right\}
$$

so that $\alpha$ is the unique real number such that $\sum_{j \geq 1} \ell_{j}^{s}$ converges absolutely for $\operatorname{Re}(s)>\alpha$ but diverges for $\operatorname{Re}(s)<\alpha$.

TheOREm 2.1 (Abscissa of convergence and Minkowski dimension; Lap2, Lap3, [Lap-vF4, Thm. 1.10]). Let $\mathcal{L}$ be an arbitrary bounded fractal string $\mathcal{L}$ having infinitely many lengths. (When $\mathcal{L}$ has finitely many lengths, it is immediate to check that $\zeta_{\mathcal{L}}$ is entire and hence, $\alpha=-\infty$ while $D=0$.) Then $\alpha=D$, the (upper) Minkowski dimension of $\mathcal{L}$ (i.e., of $\partial \Omega$, where the bounded open set $\Omega$ is any geometric realization of $\mathcal{L})$; see, respectively, (2.6) and (2.7) for the definition

\footnotetext{
${ }^{6}$ In the case of the Cantor string (or set), the Minkowski dimension exists and hence, there is not need to talk about (upper) Minkowski dimension; see 3.2 for the precise definitions.
} 
of $\alpha$ and D. In other words, the abscissa of convergence of $\zeta_{\mathcal{L}}$ and the Minkowski dimension of $\mathcal{L}$ coincide

More precisely, here, the (upper) Minkowski dimension $D=D_{\mathcal{L}}$ of $\mathcal{L}$ is the nonnegative real number given by

$$
D:=\inf \left\{\beta \geq 0: V(\varepsilon)=O\left(\varepsilon^{1-\beta}\right) \text { as } \varepsilon \rightarrow 0^{+}\right\},
$$

where

$$
V(\varepsilon)=V_{\mathcal{L}}(x):=|\{x \in \Omega: d(x, \partial \Omega)<\varepsilon\}|_{1}
$$

is the volume (or length) of the $\varepsilon$-neighborhood of the boundary $\partial \Omega$ (relative to $\Omega$ ) and $d(x, \partial \Omega)$ denotes the distance (in $\mathbb{R}$ ) from $x$ to $\partial \Omega$ Q

It follows at once from Theorem 2.1, along with the definition of $\alpha$ and $D$ respectively given in (2.6) and (2.7), that for a fractal string, we have $0 \leq D \leq 1$.

For example, for the Cantor string, the computation leading to (2.4) shows that $\alpha=\log _{3} 2$ and it is well known that $D=\log _{3} 2$, in agreement with Theorem 2.1

It is clear that the set $\mathcal{D}_{\mathcal{L}}$ of complex dimensions of a fractal string forms a discrete (and hence, at most countable) subset of $\mathbb{C}$ and (in light of Theorem 2.1. since $\zeta_{\mathcal{L}}$ is holomorphic for $\operatorname{Re}(s)>D$ )

$$
\mathcal{D}_{\mathcal{L}} \subseteq\{\operatorname{Re}(s) \leq D\},
$$

where we use the short-hand notation

$$
\{\operatorname{Re}(s) \leq D\}:=\{s \in \mathbb{C}: \operatorname{Re}(s) \leq D\},
$$

here and henceforth. (Similarly, for example, the notation $\{\operatorname{Re}(s)=D\}$ stands for the vertical line $\{s \in \mathbb{C}: \operatorname{Re}(s)=D\}$.)

The set of principal complex dimensions of $\mathcal{L}$, denoted by $\operatorname{dim}_{P C} \mathcal{L}$, is the set of complex dimensions with maximal real part:

$$
\operatorname{dim}_{P C} \mathcal{L}=\left\{\omega \in \mathcal{D}_{\mathcal{L}}: \operatorname{Re}(\omega)=D\right\} .
$$

This set (or rather, multiset) plays an important role in the general theory of complex fractal dimensions. The same is true for its counterpart in the higherdimensional theory, to be discussed in $\$ 3$,

For the Cantor string, in light of (2.5), we clearly have $\mathcal{D}_{\mathcal{L}}=\operatorname{dim}_{P C} \mathcal{L}$ but in general, $\operatorname{dim}_{P C} \mathcal{L}$ is often a strict subset of $\mathcal{D}_{\mathcal{L}}=\mathcal{D}_{\mathcal{L}}(U)$. (We implicitly assume here and in (2.9) that the connected open set $U$ is a neighborhood of the vertical line $\{\operatorname{Re}(s)=D\}$, or equivalently, of the closed half-plane $\{\operatorname{Re}(s) \geq D\}$; observe, however, that the set $\operatorname{dim}_{P C} \mathcal{L}$ itself is independent of such a choice of $U$.)

${ }^{7}$ In Lap2, Lap3, the proof of this equality relied on a result obtained in BesTa. Then, several direct proofs were given in Lap-vF2-4. See, especially, Lap-vF4 Thm. 1.10 and Thm. 13.111]; see also LapLu-vF2 ) and most recently, in LapRaZu1, §2.1.4, esp. Prop. 2.1.59 and Cor. 2.1.61], via the higher-dimensional theory of complex dimensions (to be discussed in 3 ).

${ }^{8}$ The Minkowski dimension is also called the Minkowski-Bouligand dimension [Bou], the box dimension or the capacity dimension in the literature on fractal geometry; see, e.g., Man, Fa1, MartVuo, Mat, Tri1-3], Lap1-3], Lap-vF4, LapRaZu1 and LapRaRo.

${ }^{9}$ In the present section (i.e., $\sqrt{2}$ ), for the simplicity of exposition, we will mostly ignore the distinction between upper Minkowski dimension and Minkowski dimension of $\mathcal{L}$. By contrast, in 33 we will denote, respectively, by $\bar{D}$ and $D$ these two dimensions (when the latter exists); see 3.2 for the precise definitions. Note that in the terminology of 33 the notion introduced in (2.7) is that of upper Minkowski dimension of the bounded fractal string $\mathcal{L}=\left(\ell_{j}\right)_{j \geq 1}$, viewed as the relative fractal drum (or RFD) $(\partial \Omega, \Omega)$ in $\mathbb{R}$, where $\Omega$ is any geometric realization of $\mathcal{L}$. 
We note for later use that since $\zeta_{\mathcal{L}}$ is a Dirichlet series with positive coefficients, $\zeta_{\mathcal{L}}(s) \rightarrow+\infty$ as $s \rightarrow D^{+}, s \in \mathbb{R}$ (or, more generally, as $s \in \mathbb{C}$ tends to $D$ from the right within a sector of half-angle $<\pi / 2$ and symmetric with respect to the real axis); see, e.g., Ser or Lap-vF4, §1.2]. It follows that for a fractal string (with infinitely many lengths), the half-plane $\{\operatorname{Re}(s)>D\}$ of absolute convergence of $\zeta_{\mathcal{L}}$ always coincides with the half-plane of holomorphic continuation of $\zeta_{\mathcal{L}}$, i.e., the maximal open right half-plane to which $\zeta_{\mathcal{L}}$ can be holomorphically continued. (See Lap-vF4.) Hence, in the terminology and with the notation of [LapRaZu1] (to be introduced in 33.3 , we have that $D=D_{\text {hol }}\left(\zeta_{\mathcal{L}}\right)$, the abscissa of holomorphic continuation of $\zeta_{\mathcal{L}}$.

Observe that since $D$ is always a singularity of $\zeta_{\mathcal{L}}$, then, provided $\zeta_{\mathcal{L}}$ can be meromorphically continued to a neighborhood of $D, D$ must necessarily be a pole of $\zeta_{\mathcal{L}}$ (i.e., a complex dimension of $\mathcal{L}$ ).

2.1. Fractal tube formulas. Given a fractal string $\mathcal{L}$, under suitable hypotheses 10 we can express its tube function $V(\varepsilon)=V_{\mathcal{L}}(\varepsilon)$ (or rather $\varepsilon \mapsto V(\varepsilon)$ ), as given by (2.8), in terms of its complex dimensions and the associated residues, as follows:

$$
V(\varepsilon)=\sum_{\omega \in \mathcal{D}_{\mathcal{L}}} c_{\omega} \frac{(2 \varepsilon)^{1-\omega}}{\omega(1-\omega)}+R(\varepsilon)
$$

where $c_{\omega}:=\operatorname{res}\left(\zeta_{\mathcal{L}}, \omega\right)$ is the residue of $\zeta_{\mathcal{L}}$ at $\omega \in \mathcal{D}_{\mathcal{L}}$ and $R(\varepsilon)$ is an error term which can be explicitly estimated 11 If $R(\varepsilon) \equiv 0$ (which occurs, for example, for any self-similar string if we choose $U:=\mathbb{C}$ ), the corresponding fractal tube formula (2.10) is said to be exact 12

In Lap-vF4, Ch. 8], the interested reader can find the precise statement and hypotheses of the fractal tube formula. In fact, depending, in particular, on the growth assumptions made on the geometric zeta function $\zeta_{\mathcal{L}}$, there are a variety of fractal tube formulas, with or without error term (the latter ones being called exact), as well as interpreted pointwise or distributionally; see [Lap-vF4, §8.1].

Furthermore, in the important special case of self-similar strings (of which the Cantor string is an example), even more precise (pointwise) fractal tube formulas (exact or else with an error term, depending on the goal being pursued) are obtained in Lap-vF4, §8.4].

For the example of the Cantor string (which is a self-similar string because its boundary, the ternary Cantor set, is itself a self-similar set in $\mathbb{R}$ ), we have the following exact fractal tube formula, valid pointwise for all $\varepsilon \in(0,1 / 2)$ :

$$
V_{C S}(\varepsilon)=\frac{1}{2 \log 3} \sum_{n \in \mathbb{Z}} \frac{(2 \varepsilon)^{1-D-i n \mathbf{p}}}{(D+i n \mathbf{p})(1-D-i n \mathbf{p})}-2 \varepsilon,
$$

\footnotetext{
${ }^{10}$ Namely, we assume that $\mathcal{L}$ is languid in a suitable connected open neighborhood $U$ of $\{\operatorname{Re}(s) \geq D\}$; i.e., roughly speaking, $\zeta_{\mathcal{L}}$ can be meromorphically continued to $U$ and satisfies a suitable polynomial growth condition for a screen $S$ bounding $U$ (in the sense of [Lap-vF4 §5.3]).

${ }^{11}$ In this discussion, for clarity, we assume implicitly that all of the complex dimensions are simple (i.e., are simple poles of $\zeta_{\mathcal{L}}$ ). In the general case, (2.10) should be replaced by

$$
V(\varepsilon)=\sum_{\omega \in \mathcal{D}_{\mathcal{L}}} \operatorname{res}\left(\frac{(2 \varepsilon)^{1-s}}{s(1-s)} \zeta_{\mathcal{L}}(s), \omega\right)+R(\varepsilon) .
$$

${ }^{12}$ More generally, we obtain an exact tube formula whenever $\mathcal{L}$ (i.e., $\zeta_{\mathcal{L}}$ ) is strongly languid (which implies that $U:=\mathbb{C}$ ), in the sense of Lap-vF4 §5.3].
} 
with $D:=\log _{3} 2$ and $\mathbf{p}:=2 \pi / \log 3$.

Observe that we can rewrite (2.12) in the following form:

$$
V_{C S}(\varepsilon)=\varepsilon^{1-D} G\left(\log _{3} \varepsilon^{-1}\right)-2 \varepsilon
$$

where $G$ is a nonconstant, positive 1-periodic function on $\mathbb{R}$ which is bounded away from zero and infinity. In fact,

$$
0<\mathcal{M}_{*}=\min _{u \in \mathbb{R}} G(u) \text { and } \mathcal{M}^{*}=\max _{u \in \mathbb{R}} G(u)<\infty,
$$

where $\mathcal{M}_{*}$ and $\mathcal{M}^{*}$ denote, respectively, the lower and upper Minkowski content of $\mathcal{L}$, defined by 13

$$
\mathcal{M}_{*}:=\liminf _{\varepsilon \rightarrow 0^{+}} \varepsilon^{-(1-D)} V_{C S}(\varepsilon)
$$

and

$$
\mathcal{M}^{*}:=\limsup _{\varepsilon \rightarrow 0^{+}} \varepsilon^{-(1-D)} V_{C S}(\varepsilon) .
$$

(Clearly, we have that $0 \leq \mathcal{M}_{*} \leq \mathcal{M}^{*} \leq \infty$.) For the Cantor string,

$$
\mathcal{M}_{*}=2^{1-D} D^{-D} \approx 2.4950 \text { and } \mathcal{M}^{*}=2^{2-D} \approx 2.5830 \text {. }
$$

Hence, $\mathcal{M}_{*}<\mathcal{M}^{*}$ and thus, the limit of $V(\varepsilon) / \varepsilon^{1-D}$ as $\varepsilon \rightarrow 0^{+}$does not exist; i.e., the Cantor string (and hence, also the Cantor set) is not Minkowski measurable 14

Recall that a fractal string $\mathcal{L}$ (or its boundary $\partial \Omega$ ) is said to be Minkowski measurable if the above limit exists in $(0,+\infty)$ and then,

$$
\mathcal{M}:=\lim _{\varepsilon \rightarrow 0^{+}} \varepsilon^{-(1-D)} V(\varepsilon)
$$

is called the Minkowski content of $\mathcal{L}$ (or of $\partial \Omega$ ). In other words, $\mathcal{L}$ is Minkowski measurable if $\mathcal{M}_{*}=\mathcal{M}^{*}$, and this common value, denoted by $\mathcal{M}$, lies in $(0,+\infty)$.

There is another way to show that $\mathcal{L}$ (in the present case, $\mathcal{L}=\mathcal{L}_{C S}$, the Cantor string) is not Minkowski measurable. This can be seen by using the principal complex dimensions of $\mathcal{L}$, as defined by (2.9); in other words, the complex dimensions with maximal real part $D$. Indeed, the following useful Minkowski measurability criterion was obtained in Lap-vF1-4.

Theorem 2.2 (Minkowski measurability and complex dimensions; Lap-vF4, Thm. 8.15]). Under suitable hypotheses 15 the following statements are equivalent:

(i) $\mathcal{L}$ is Minkowski measurable (with Minkowski dimension $D \in(0,1)$ ).

(ii) The only principal complex dimension of $\mathcal{L}$ is $D$ itself, and it is simple.

Observe that for the Cantor string $\mathcal{L}_{C S}$, there are infinitely many complex conjugate nonreal complex dimensions with real part $D$. Furthermore, $D=\log _{3} 2$ (like each of the complex dimensions of $\mathcal{L}_{C S}$ in (2.5)) is simple; i.e., it is a simple pole of $\zeta_{C S}$. Therefore, this yields another proof of the fact that $\mathcal{L}_{C S}$ (or, equivalently, the Cantor set $C$ ) is not Minkowski measurable.

\footnotetext{
${ }^{13}$ An entirely analogous definition of $\mathcal{M}_{*}$ and $\mathcal{M}^{*}$ can be given for any fractal string $\mathcal{L}$; simply replace $V_{C S}(\varepsilon)$ by $V(\varepsilon)=V_{\mathcal{L}}(\varepsilon)$ in (2.14) and 2.15), respectively.

${ }^{14}$ This fact was first established in LapPo1-2, by using a direct computation and Theorem 2.3 and then extended in Lap-vF2-4 to a whole class of examples (including lattice self-similar strings and generalized Cantor strings; see Lap-vF4, §8.4.2 and §10.1]). Another, more conceptual, proof was given in Lap-vF4 Ch. 8] by using the existence of nonreal principal complex dimensions of the Cantor string; see Theorem 2.2 and the comments following it.

${ }^{15}$ In essence, we assume that $\mathcal{L}$ is languid (in the sense of footnote 10) for a screen $S$ passing between the vertical line $\{\operatorname{Re}(s)=D\}$ and all of the complex dimensions of $\mathcal{L}$ with real part $<D$.
} 
There is another, very useful, characterization of Minkowski measurability, obtained in LapPo2 and announced in LapPo1.

Theorem 2.3 (Minkowski measurability and fractal strings; [LapPo2]). Let $\mathcal{L}=\left(\ell_{j}\right)_{j=1}^{\infty}$ be an arbitrary fractal string (of Minkowski dimension $D \in(0,1)$ ). Then, the following statements are equivalent:

(i) $\mathcal{L}$ is Minkowski measurable.

(ii) $\ell_{j} \sim L j^{-1 / D}$ as $j \rightarrow \infty$, for some constant $L \in(0,+\infty) 16$

In this case, the Minkowski content $\mathcal{M}$ of $\mathcal{L}$ is given by

$$
\mathcal{M}=\frac{2^{1-D}}{1-D} L^{D}
$$

REMARK 2.4. (a) The proof of Theorem 2.3 given in LapPo2 is analytical. Later, a different approach to a part of that proof was taken by Kenneth Falconer in [Fa2], based on a suitable dynamical system, and more recently, by Jan Rataj and Steffen Winter in RatWi], based on aspects of geometric measure theory.

(b) If $\zeta_{\mathcal{L}}$ has a meromorphic continuation to a neighborhood of $D$ and either condition (i) or (ii) of Theorem 2.3 is satisfied (or certainly, if the hypotheses and either condition $(i)$ or $(i i)$ of Theorem 2.2 hold), then

$$
\mathcal{M}=\frac{2^{1-D}}{D(1-D)} \operatorname{res}\left(\zeta_{\mathcal{L}}, D\right)
$$

(c) Even though the Cantor string $\mathcal{L}_{C S}$ is not Minkowski measurable, it is the case that its average Minkowski content, $\mathcal{M}_{a v}$, defined as a suitable Cesaro average of $V_{C S}(\varepsilon) \varepsilon^{-(1-D)}$ (see the $N=1$ case of footnote 65), exists and can be explicitly computed in terms of $\operatorname{res}\left(\zeta_{\mathcal{L}}, D\right)$; see Lap-vF4, §8.4.3]. The same is true for any lattice self-similar string; see $[$ Lap-vF4, Thm. 8.23] 17 More specifically, a lattice self-similar string is not Minkowski measurable but its average Minkowski content, $\mathcal{M}_{a v}$, exists in $(0,+\infty)$ and is also given by the right-hand side of (2.18); see Lap-vF4, Thm. 8.30].

(d) More generally, a self-similar string is Minkowski measurable if and only if it is nonlattice. In this case, its Minkowski content, $\mathcal{M}$, is given by either (2.17) or (2.18); see Lap-vF4, Thms. 8.23 and 8.36]. Further, we have $\mathcal{M}_{a v}=\mathcal{M}$, since there is no need to take any averaging anymore.

2.2. Other examples of fractal explicit formulas. Let $\mathcal{L}=\left(\ell_{j}\right)_{j \geq 1}$ be a fractal string. Then, it is a vibrating object and its (normalized frequency) spectrum consists of the numbers $f_{j, n}=n \cdot \ell_{j}^{-1}$, where $n, j \in \mathbb{N}=\{1,2, \cdots\}$. One can think of $\mathcal{L}$ as being composed of infinitely many ordinary Sturm-Liouville strings, with lengths $\ell_{j}$, vibrating independently of one another and with their endpoints fixed (i.e., corresponding to homogeneous Dirichlet boundary conditions for the one-dimensional Laplacian $-d^{2} / d x^{2}$ on the open set $\Omega \subseteq \mathbb{R}$ ).

One of the major themes of fractal string theory is the study of the interplay between the geometry and the spectra of fractal strings.

\footnotetext{
${ }^{16}$ Here, $\ell_{j} \sim m_{j}$ as $j \rightarrow \infty$ means that $\ell_{j} / m_{j} \rightarrow 1$ as $j \rightarrow \infty$.

${ }^{17}$ The precise definition of (bounded) self-similar strings is given in Lap-vF4. Ch. 2]. Here, we simply recall that a self-similar string is said to be lattice if its distinct scaling ratios generate a (multiplicative) group of rank 1. It is said to be nonlattice, otherwise. The detailed structure of the complex dimensions of self-similar strings is discussed in [Lap-vF4. Chs. 2 and 3].
} 
Let $N_{\mathcal{L}}$ be the geometric counting function of $\mathcal{L}$, given by (here, \#A denotes the cardinality of a finite set $A$ )

$$
N_{\mathcal{L}}(x)=\#\left\{j \geq 1: \ell_{j}^{-1} \leq x\right\}, \text { for } x>0 .
$$

Similarly, let $N_{\nu}$ denote the (frequency or) spectral counting function of $\mathcal{L}$ :

$$
N_{\nu}(x)=\#\{f: f \text { is a frequency of } \mathcal{L} \text {, with } f \leq x\} \text {, for } x>0 .
$$

Then, $N_{\mathcal{L}}$ and $N_{\nu}$ are connected via the following identity, for all $x>0.18$

$$
N_{\nu}(x)=\sum_{j=1}^{\infty} N_{\mathcal{L}}\left(\frac{x}{j}\right)
$$

Essentially equivalently, the geometric and spectral zeta functions $\zeta_{\mathcal{L}}$ and $\zeta_{\nu}$ of $\mathcal{L}$ are connected by the following key identity (first observed in Lap2, Lap3).19

$$
\zeta_{\nu}(s)=\zeta(s) \cdot \zeta_{\mathcal{L}}(s)
$$

where $\zeta$ denotes the classic Riemann zeta function, initially defined by $\zeta(s):=$ $\sum_{n=1}^{\infty} n^{-s}$ for $\operatorname{Re}(s)>1$ and then meromorphically continued to all of $\mathbb{C}$ (see, e.g., Edw, Pat, Tit]).

Note that in order to apprehend the principal complex dimensions of $\mathcal{L}$ and their effect on the spectrum of $\mathcal{L}$, one must work in the closed critical strip $\{0 \leq$ $\operatorname{Re}(s) \leq 1\}$ of $\zeta$ or, if one excludes the extreme cases when $D=0$ or $D=1$, in its open counterpart, $\{0<\operatorname{Re}(s)<1\}$, henceforth referred to as the critical strip.

Now, let us give a few examples of fractal explicit formulas, analogous to the fractal tube formulas discussed in 2.1 above. In the spirit of this overview, we will not strive here for either mathematical precision or for the most general statements but instead refer to [ap-vF4, Chs. 5 and 6] for all of the details and a much broader perspective.

Assume, for clarity, that all of the complex dimensions of $\mathcal{L}=\left(\ell_{j}\right)_{j=1}^{\infty}$ are simple. Then, under appropriate hypotheses, we obtain the following pointwise or distributional explicit formulas with error terms 20

$$
N_{\mathcal{L}}(x)=\sum_{\omega \in \mathcal{D}_{\mathcal{L}}} c_{w} \frac{x^{\omega}}{\omega}+R_{\mathcal{L}}(x)
$$

and

$$
N_{\nu}(x)=\zeta_{\mathcal{L}}(1) x+\sum_{\omega \in \mathcal{D}_{\mathcal{L}}} c_{\omega} \zeta(\omega) \frac{x^{\omega}}{\omega}+R_{\nu}(x)
$$

where, as before, $c_{\omega}:=\operatorname{res}\left(\zeta_{\mathcal{L}}, \omega\right)$ for every $\omega \in \mathcal{D}_{\mathcal{L}}$ and $R_{\mathcal{L}}$ and $R_{\nu}$ are error terms which can be suitably estimated (either pointwise or distributionally); see Lap-vF4, §6.2]. (Note that $\zeta_{\mathcal{L}}(1)=|\Omega|_{1}=\sum_{j=1}^{\infty} \ell_{j}$, the total length of the fractal string $\mathcal{L}$.)

Analogous formulas, now necessarily interpreted distributionally rather than pointwise, can be obtained for the positive measures $\eta$ and $\nu$, respectively defined

\footnotetext{
${ }^{18}$ Note that for each fixed $x>0$, the sum in (2.21) contains only finitely many nonzero terms. However, as $x \rightarrow+\infty$, the number of these terms tends to $+\infty$.

${ }^{19}$ Here, $\zeta_{\nu}(s)$ is given for $\operatorname{Re}(s)>1$ by $\zeta_{\nu}(s):=\sum_{f} f-s$, where $f$ ranges through all the (normalized) frequencies of $\mathcal{L}$, and is then meromorphically continued wherever possible.

${ }^{20}$ Under somewhat stronger assumptions, we obtain exact formulas; namely, either $R_{\mathcal{L}}(x) \equiv 0$ or (more rarely) $R_{\nu}(x) \equiv 0$.
} 
as $d N_{\mathcal{L}} / d x$ and $d N_{\nu} / d x$ (the distributional derivatives of $N_{\mathcal{L}}$ and $N_{\nu}$ ) and referred to as the geometric and spectral densities of states; see [Lap-vF4, §6.3.1]. Alternatively,

$$
\eta([0, x]):=\frac{N_{\mathcal{L}}([0, x])+N_{\mathcal{L}}([0, x))}{2}, \text { for all } x>0,
$$

and similarly for $\nu$ and $N_{\nu}$.

REMARK 2.5. (Fractal string theory and its ramifications.) Fractal string theory and the associated theory of complex dimensions has been developed in many directions and applied to a variety of fields, including harmonic analysis, fractal geometry, number theory and arithmetic geometry, complex analysis, spectral geometry, geometric measure theory, probability theory, nonarchimedean analysis, operator algebras and noncommutative geometry, as well as dynamical systems and mathematical physics.

In particular, in Lap-vF4, Ch. 13], are discussed a variety of extensions or applications of fractal string theory in diverse settings (prior to the development of the higher-dimensional theory of complex dimensions and of fractal zeta functions in LapRaZu1-10, to be partly surveyed in $\oint_{3}$ ), including fractal tube formulas for fractal sprays (especially, self-similar sprays and tilings), in Lap-vF4, §12.1] (based on LapPe2-3, LapPeWi1-2, $\mathbf{P e},[\mathbf{P e W} \mathbf{i})$, complex dimensions and fractal tube formulas for $p$-adic fractal (and self-similar) strings, in Lap-vF4, §12.2] (based on LapLu1-3, LapLu-vF1-2 ), multifractal zeta functions and strings, in Lap-vF4, \$12.3] (based on LapRo, LapLevyRo, ElLapMcRo), random fractal strings and zeta functions, in [Lap-vF4 $\S 12.4]$ (based on HamLap]), as well as fractal membranes (or 'quantized fractal strings') and their associated moduli space, in Lap-vF4, §12.5] (based on Lap7, LapNes). See also Lap-vF4, §12.2.1] for a brief description of a first direct attempt at a higher-dimensional theory, in LapPe1, where a fractal tube formula was obtained for the Koch snowflake curve via a direct computation.

In addition to the aforementioned articles, we refer the interested reader to the research books [Lap-vF2-4, Lap7, LapRaZu1, HerLap1, Lap10, as well as Lap-vF6, CaLapPe-vF and LapRaRo, along with the research papers and survey articles Cae, CobLap1-2, CranMH, DemDenKoü, DemKoÖÜ, DenKoÖÜ], DenKoÖRய̈], deSLapRRo, DubSep, Es1-2, [EsLi1-2, [Fa2, Fr, FreiKom, Gat, Ger], GerScm1-2, HeLap, HerLap2-5, KeKom, Kom, KomPeWi], KoRati], LalLap1-2, Lap1-6, Lap8-9, LapMa1-2, LapPo1-3, LapRaZu2-10, LapRoZu, Lap-vF1, Lap-vF5, Lap-vF7, LapWat, LevyMen, LiRadz, MorSep, MorSepVi1-2], Ol1-2], Ra1-2], RatWi], Tep1-2, [vF1-2], Wat and [Zu1-2], for various aspects of fractal string theory and its applications.

2.3. Analogy with Riemann's explicit formula. One of the most beautiful formulas in mathematics, in the author's opinion, is Riemann's explicit formula. The latter connects the prime number counting function

$$
\Pi_{\mathcal{P}}(x):=\#\{p \in \mathcal{P}: p \leq x\}, \text { for } x>0
$$

(where $\mathcal{P}$ denotes the set of prime numbers) and the zeros of the Riemann zeta function. Because it is simpler to state (as well as to justify), although a precise proof was provided only about forty years after the publication of Riemann's celebrated 1858 paper Rie, we will state a modern form of this formula. Namely, 
consider the weighted prime powers counting function

$$
\varphi(x):=\sum_{p^{n} \leq x} \frac{1}{n}, \text { for } x>0,
$$

where the sum ranges over all prime powers $p^{n}$ (counted with a weight $1 / n$, for every $n \in \mathbb{N}$ ). Then

$$
\varphi(x)=L i(x)-\sum_{\rho} L i\left(x^{\rho}\right)+\int_{x}^{+\infty} \frac{1}{t^{2}-1} \frac{d t}{t \log t}-\log 2,
$$

where $\operatorname{Li}(x):=\int_{0}^{x} \frac{d t}{\log t}$ is the integral logarithm and the infinite sum in (2.27) is taken over all of the critical zeros $\rho$ of $\zeta$ (in $\{0<\operatorname{Re}(s)<1\}$ ), while the negative of the integral in (2.27) corresponds to the same sum but now taken over the trivial zeros $-2,-4,-6, \cdots$ of $\zeta$. Furthermore, the leading term, $L i(x)$, corresponds to the (simple) pole of $\zeta=\zeta(s)$ at $s=1$.

It follows from this formula (combined with an appropriate analysis, for instance based on a Tauberian theorem) that 21

$$
\Pi_{\mathcal{P}}(x) \sim \operatorname{Li}(x) \quad \text { as } x \rightarrow+\infty,
$$

or equivalently, that

$$
\Pi_{\mathcal{P}}(x) \sim \frac{x}{\log x} \quad \text { as } x \rightarrow+\infty,
$$

which is the statement of the famous Prime Number Theorem (PNT), as conjectured independently by Gauss (1792) and Legendre (1797) and proved indepen-

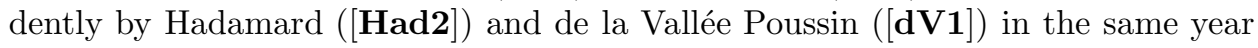
(1896), but a century later. Note, however, that it also took about forty years after the publication of [Rie in 1858 in order to prove PNT, in the form of (2.28), about the same amount of time it took to rigorously justify Riemann's original explicit formula; see van Mangoldt's work vM1-2, along with Ingham's book Ing 22 The latter formula is obtained by first proving (2.27) and then by using Möbius inversion Edw, Ove as follows (with $\varphi$ now given by the explicit formula (2.27)):

$$
\Pi_{\mathcal{P}}(x)=\sum_{n=1}^{\infty} \frac{\mu(n)}{n} \varphi\left(x^{1 / n}\right)
$$

where $\mu$ denotes the Möbius function defined on $\mathbb{N}$ by $\mu(n)=(-1)^{k}$ if $n \geq 2$ is a product of $k$ distinct primes, $\mu(1)=1$, and $\mu(n)=0$ otherwise. Riemann's original explicit formula is then deduced by substituting into (2.30) the expression of $\varphi$ given by (2.27).

The analogy between Riemann's explicit formula (in any of its various disguises) and the fractal explicit formulas discussed in 22.1 and $\$ 2.2$ is now apparent. The (critical) zeros of $\zeta$ correspond to the (nonreal) complex dimensions of $\mathcal{L}$, while the prime counting function $\Pi_{\mathcal{P}}$ in $(2.25)$ (or the weighted prime powers counting

\footnotetext{
${ }^{21}$ The expression $f(x) \sim g(x)$ as $x \rightarrow+\infty$ means that $f(x) / g(x) \rightarrow 1$ as $x \rightarrow+\infty$.

${ }^{22}$ There are more direct (but less insightful) ways to prove PNT. They also typically require to know that $\zeta(s)$ does not have any zero on the vertical line $\{\operatorname{Re}(s)=1\}$ (Hadamard, Had1, 1893). However, in order to obtain a version of 2.28) with error term (PNT with error term), the Riemann-von Mangoldt explicit formula 2.27) (or one of its counterparts) is the most reliable tool (combined, for example, with an appropriate Tauberian theorem); see dV2 along, e.g., with [Edw (for a detailed history and analysis of Riemann's paper, Rie]) and, especially, Ing, Ivi, KarVo, Pat, Tit].
} 
function $\varphi$ in (2.26) ) corresponds to the geometric or spectral counting function $N_{\mathcal{L}}$ or $N_{\nu}$ (e.g., in (2.23) or (2.24), respectively), or else (in a more sophisticated but also more geometric form) to the tube function (or distribution) $V(\varepsilon)$ in (2.10).

In particular, the oscillations (in the counting function of the primes) associated with the (critical) zeros of $\zeta$ in the infinite sum appearing in (2.27) or in (2.30) correspond to the geometric oscillations (in the geometric counting function $N_{\mathcal{L}}$ in (2.23) or in the tube function $V(\varepsilon)$ in (2.10) ) or to the spectral oscillations (in the frequency counting function $N_{\nu}$ in (2.24)). We will further discuss these oscillations in $\$ 2.4$

At this stage, it is natural for the reader to be troubled by the presence of zeros in (2.27) or (2.30), as opposed to just poles (or "complex dimensions") in (2.23), (2.24) and (2.10). However, this apparent discrepancy is quickly resolved by noting that the (necessarily simple) poles of (minus) the logarithmic derivative $-\zeta^{\prime}(s) / \zeta(s)$ of $\zeta(s)$ correspond precisely to the zeros of $\zeta(s)$ and to its only pole (at $s=1$, which accounts for the leading term $\operatorname{Li}(x)$ in (2.27) $)$. In addition, the residue of $-\zeta^{\prime}(s) / \zeta(s)$ at a pole $s=\omega$ is a nonzero integer whose sign tells us whether it corresponds to a zero or a pole (here, $s=1)$ of $\zeta(s)$, and whose absolute value is the multiplicity of the zero or pole. As a simple exercise, the reader may wish to verify this statement and determine which sign of the residue corresponds to a zero or a pole.

In closing this subsection, we point out that the (pointwise or distributional) explicit formulas obtained in Lap-vF4, Ch. 5], with or without error term, and used throughout Lap-vF4, esp., in Chs. 6-11], extend Riemann's explicit formula (and its known number-theoretic counterparts) to a fractal, geometric, spectral, or dynamical setting in which the corresponding zeta functions do not necessarily have an Euler product or satisfy a functional equation. Furthermore, the general framework within which these explicit formulas are developed is sufficiently broad and flexible in order for the resulting formulas to be applied to a variety of situations (including arithmetic ones) and to help unify, in the process, aspects of fractal geometry and number theory, both technically and conceptually 23

2.4. The meaning of complex dimensions. In light of the fractal tube formulas and the other fractal explicit formulas discussed in $\$ 2.2$ and $\$ 2.3$, the following intuition of the notion of complex dimensions can easily be justified, mathematically. The real parts of the complex dimensions correspond to the amplitudes of 'geometric waves' (propagating through the 'space of scales'), while the imaginary parts of the complex dimensions correspond to the frequencies of those waves.

An analogous interpretation can be given in the spectral setting and in the dynamical setting. A common thread to these interpretations is provided by the (generalized) explicit formulas of Lap-vF4 mentioned at the end of $\$ 2.3$ Associated key words are oscillations, vibrations, and wave-like phenomena, which could also be applied to the number-theoretic setting corresponding to Riemann's explicit formula for the prime number counting function and discussed in $\$ 2.3$.

The author has conjectured since the early 1990s that (possibly generalized or even virtual) fractal geometries and arithmetic geometries pertained to the same mathematical realm. Consequently, there should exist a fractal-like geometry whose

${ }^{23}$ The interested reader can find in Lap-vF4 $\$ 5.1 .1$ and $\left.\S 5.6\right]$ many references about numbertheoretic and analytic explicit formulas in a variety of contexts, including Wei4-5, Den1-3. DenSchr, Har1-3. 
complex dimensions are the Riemann zeros (the critical zeros of $\zeta=\zeta(s)$ ); see, especially, the author's book Lap7. There, in particular, an extension (and 'quantization') of the notion of fractal string is introduced and coined 'fractal membrane'. It turns out to be a noncommutative space, in the sense of [Con1. The associated moduli space of fractal membranes (which can be thought of physically as a quantization of the moduli space of fractal strings) plays a fundamental role in Lap7 in order to provide a conjectural explanation of why the Riemann hypothesis should be true, both for the classic Riemann zeta function and for all number-theoretic zeta functions (or $L$-functions, ParsSh1-2, Sarn, Lap-vF4, App. A], Lap7, Apps. B, C \& E]) occurring in arithmetic geometry. It is expressed in terms of a (still conjectural) noncommutative dynamical system on the moduli space of fractal membranes, as well as of its counterparts on the associated moduli spaces of zeta functions (or 'partition functions') and of divisors (i.e., zeros and poles) on the Riemann sphere (which is the natural realm of the Riemann zeros and more generally, of the complex fractal dimensions). See, especially, Lap7, Ch. 5].

2.5. Fractality, complex dimensions and irreality. Since, as we have seen, the imaginary parts of the complex dimensions give rise to oscillations in the intrinsic geometry (or in the spectra) of fractal strings, it is natural to wonder whether one could not define the elusive notion of fractality in terms of complex dimensions.

In Lap-vF1-4 (as well as later, in higher dimensions, in LapRaZu1 to be discussed in $\$ 3$ below), an object is said to be 'fractal' if it has at least one nonreal complex dimension 24 and hence, in other words, according to the explicit formulas discussed in $\S \$ 2.12 .4$ (when $N=1$ ) and in $\$ 3.5$ (when $N \geq 1$ is arbitrary, where $N$ is the dimension of the embedding space), if it has intrinsic geometric, spectral, dynamical or arithmetic oscillations 25

In the case of a fractal string, the complex dimensions are the poles of the associated geometric zeta functions, whereas (anticipating on the discussion of LapRaZu1 given in \$3), in higher dimensions (i.e., for bounded subsets of $\mathbb{R}^{N}$ or, more generally, for relative fractal drums in $\mathbb{R}^{N}$, for any $N \geq 1$ ), the complex dimensions are the poles of the associated fractal zeta functions. Furthermore, as was alluded to near the end of $\$ 2.3$, in the arithmetic setting, the role played by the complex dimensions in fractal geometry is now essentially played by the Riemann zeros, or by their more general number-theoretic analogs (e.g., the critical zeros of automorphic $L$-functions or of zeta functions of varieties over finite fields).

REMARK 2.6. (Reality principle.) Geometrically, the fact that the nonreal complex dimensions come in complex conjugate pairs is significant. This is what enables us, for instance, to obtain a real-valued (and even positive) expression for the tube function $V(\varepsilon)$ in the fractal tube formulas of $\$ 2.126$ For example, the fractal tube

\footnotetext{
${ }^{24}$ Since nonreal complex dimensions come in complex conjugate pairs, a fractal-like object must have at least two complex conjugate nonreal complex dimensions. In fact, in the geometric setting, it typically has infinitely many nonreal complex conjugate pairs of them.

${ }^{25}$ For various examples for which the source of the oscillations is of a dynamical (respectively, spectral) nature, see Lap-vF4. Ch. 7 and §12.5.3] (respectively, Lap-vF4. Chs. 6 and 9-11]), while for the case when it is of a geometric (respectively, arithmetic) nature, see Lap-vF4 Chs. 6 and 9-13] (respectively, Lap-vF4, Chs. 9 and 11]).

${ }^{26}$ An analogous comment can be made about the geometric and spectral counting functions of $\$ 2.2$ or even the prime numbers counting functions of $\$ 2.3$ (provided the "complex dimensions"
} 
formula for the Cantor string in (2.12) can be written as follows (with $D:=\log _{3} 2$ and $\mathbf{p}:=2 \pi / \log 3)$ :

$$
V_{C S}(\varepsilon)=\frac{2^{-D} \varepsilon^{1-D}}{D(1-D) \log 3}+(2 \varepsilon)^{1-D}\left(\sum_{n=1}^{\infty} \operatorname{Re}\left(\frac{(2 \varepsilon)^{-i n \mathbf{p}}}{(D+i n \mathbf{p})(D-i n \mathbf{p})}\right)\right)-2 \varepsilon,
$$

which in turn can be further expressed in terms of real-valued functions involving sine and cosine, by using Euler's identity

$$
(2 \varepsilon)^{-i n \mathbf{p}}=\cos (n \mathbf{p} \log 2 \varepsilon)-i \sin (n \mathbf{p} \log 2 \varepsilon), \text { for all } n \in \mathbb{Z} .
$$

It is obvious that the Cantor string $\mathcal{L}_{C S}$ (and hence, also the Cantor set) is fractal according to the above definition. Indeed, in light of (2.5), it has infinitely many complex conjugate pairs of nonreal (principal) complex dimensions, $D \pm i n \mathbf{p}$, with $n \in \mathbb{N}$ (as well as with $D$ and $\mathbf{p}$ as in Remark 2.6). This is also apparent in the fractal tube formula (2.12) (or its "real" form (2.31) in Remark 2.6), as well as in the following pointwise explicit formulas for $N_{C S}$ and $N_{\nu, C S}$, the geometric and spectral counting functions of $\mathcal{L}_{C S}$, respectively 27

$$
N_{C S}(x)=\frac{1}{2 \log 3} \sum_{n \in \mathbb{Z}} \frac{x^{D+i n \mathbf{p}}}{D+i n \mathbf{p}}-1
$$

and

$$
N_{\nu, C S}(x)=x+\frac{1}{2 \log 3} \sum_{n \in \mathbb{Z}} \zeta(D+i n \mathbf{p}) \frac{x^{D+i n \mathbf{p}}}{D+i n \mathbf{p}}+O(1) .
$$

It is shown in [Lap-vF4, Ch. 11] that the Riemann zeta function $\zeta=\zeta(s)$ and many other arithmetic zeta functions (as well as other Dirichlet series) cannot have infinite vertical arithmetic progressions of critical zeros 28 It follows that the spectral oscillations in (2.34), just like the geometric oscillations in (2.33), subsist. In fact, this result is established by reasoning by contradiction and using the counterparts of the explicit formulas (2.33) and (2.34), as well as by proving that (virtual) generalized Cantor strings always have both geometric and spectral oscillations of a suitable kind (namely, of leading order $x^{D}$ as $x \rightarrow+\infty$ ); see Lap-vF4, Ch. 10]. The sought for contradiction is then reached by making the nonreal complex dimensions $D+i n \mathbf{p}$ (with $n \in \mathbb{Z} \backslash\{0\}$ ) of the (virtual, generalized) Cantor string coincide with the presumed zeros of $\zeta$ in infinite arithmetic progression along the vertical line $\{\operatorname{Re}(s)=D\}$. (Here, $D \in(0,1)$ and the period $\mathbf{p}>0$ can be chosen to be arbitrary. 29 Then, in light of the counterpart of (2.34) in this context, we deduce that $N_{\nu}$ does not have any oscillations of leading order $x^{D}$, in contradiction

are interpreted there as the zeros and the pole(s) of $\zeta=\zeta(s)$ or of its counterpart; that is, as the poles of (minus) the logarithmic derivative of the (arithmetic) zeta function under consideration.

${ }^{27}$ See Lap-vF4 Eqns. (1.31) and (6.57)], where in the latter equations, we have slightly adapted the formula because our Cantor string (unlike in Lap-vF4 Ch. 2]) has total length 1 (rather than 3). Note that (2.33) is an exact explicit formula whereas (2.34) has an error term.

${ }^{28}$ Unknown to the authors of [Lap-vF1-2] at the time, C. R. Putnam [Put1-2] had established a similar result in the case of $\zeta=\zeta(s)$, by completely different methods which only extended to a few arithmetic zeta functions.

${ }^{29}$ This is why the corresponding Cantor strings are not geometric, in general, but instead generalized (and virtual) fractal strings, in the sense of [Lap-vF4 Chs. 4 and 10-11]. 
to what is claimed just above. Observe the analogy with the method of proof (outlined in the latter part of 2.6 .2 below) of a key result of LapMa2 connecting the Riemann hypothesis and inverse spectral problems for fractal strings.

REMARK 2.7. (Multiplicative oscillations.) In order to better understand the nature of the (multiplicative) oscillations intrinsic to 'fractality', as expressed by fractal explicit formulas and the presence of nonreal complex dimensions (necessarily in complex conjugate pairs), it is helpful to first consider the following simple situation. Think, for instance, that each term of the form $x^{z}$ (with $x>0$ and $z \in \mathbb{C}$ such that $z=d+i \tau$, where $d \in \mathbb{R}$ and $\tau>0$, say) arising in a given fractal (or arithmetic) explicit formula can be written as follows:

$$
x^{z}=x^{d} x^{i \tau}=x^{d}(\cos (\tau \log x)+i \sin (\tau \log x)) .
$$

Now, clearly, the real part $d$ of $z$ governs the amplitude of the oscillations while the imaginary $\tau$ of $z$ governs the frequency of the oscillations. Observe that physically, the term $x^{z}$ can be viewed as a multiplicative analog of a plane wave (or a standing wave). The different terms of the form $x^{z}$ (or $\varepsilon^{-z}$ ) occurring in the infinite sum ranging over all of the visible complex dimensions and appearing in a given fractal explicit formula (or fractal tube formula), such as (2.23), (2.24), (2.33), (2.34) (or (2.10), (2.12), (2.31)) provide a whole spectrum of amplitudes and frequencies associated with the corresponding superposition of 'standing waves'. The fact that these waves arise in scale space (rather than in ordinary frequency or momentum space, in physicists' terminology), explains why the corresponding oscillations are viewed multiplicatively (rather than additively) here.

This is very analogous to what happens for Fourier series. Observe, however, that unlike for Fourier series, the frequencies are no longer, in general, multiple integers of a given fundamental frequency 30 In addition, the varying amplitudes of the oscillations do not have a counterpart for ordinary Fourier series. If one replaces the classic theory of Fourier series by Harald Bohr's less familiar but more general theory of almost periodic functions Boh (usually associated with purely imaginary rather than with arbitrary complex numbers $z$ ), one is getting closer to improving one's understanding of the situation. Nevertheless, the fact that typically, the complex dimensions form a countably infinite and discrete subset of $\mathbb{C}$ (rather than of $\mathbb{R}$ ) adds a lot of complexity and richness to the corresponding generalized 'almost periodic' functions (or distributions, [Katzn]).

EXAMPLE 2.8. (The a-string.) For a simple example of a string that is not fractal, in the above sense, consider the $a$-string, where $a>0$ is arbitrary 31 Thus,

$$
\Omega=\Omega_{a}:=[0,1] \backslash\left\{j^{-a}: j \in \mathbb{N}\right\}=\bigcup_{j=1}^{\infty}\left((j+1)^{-a}, j^{-a}\right)
$$

and hence,

$$
\partial \Omega=\partial \Omega_{a}=\left\{j^{-a}: j \in \mathbb{N}\right\} \cup\{0\},
$$

\footnotetext{
${ }^{30}$ In that sense, the Cantor string and more generally, all lattice self-similar strings, are rather exceptional. In contrast, the complex dimensions of (bounded or unbounded) nonlattice self-similar strings have a much richer quasiperiodic structure; see Lap-vF4 Ch. 3].

${ }^{31}$ The $a$-string, then viewed in Lap1 as a one-dimensional fractal drum, was the first example of fractal string (before that notion was formalized in $[\mathbf{L a p P o 2}$ ) and was used in Lap1. Exple. 5.1 and Exple. 5.1'] to show that the remainder estimates obtained in Lap1 for the spectral asymptotics of fractal drums are sharp, in general (and in every possible dimension).
} 
while

$$
\mathcal{L}=\mathcal{L}_{a}=\left(j^{-a}-(j+1)^{-a}\right)_{j=1}^{\infty} .
$$

It is shown in Lap-vF4 Thm. 6.21] that the geometric zeta function $\zeta_{\mathcal{L}_{a}}$ of $\mathcal{L}_{a}$ admits a meromorphic continuation to all of $\mathbb{C}$ and that the complex dimensions of $\mathcal{L}_{a}$ are all simple with

$$
\mathcal{D}_{\mathcal{L}_{a}}=\{D,-D,-2 D,-3 D, \cdots\}
$$

where $D=1 /(a+1)$ is the Minkowski dimension of $\mathcal{L}_{a}$ (or equivalently, of $\partial \Omega_{a}$ ) 32, Note that $D \in(0,1)$ whereas $H=0$ for any $a>0$, where $H$ is the Hausdorff dimension of the compact set $\partial \Omega_{a} \subseteq \mathbb{R}$. This illustrates the fact that the Minkowski dimension, and not the Hausdorff dimension, is the proper notion of real dimension pertaining to the theory of complex fractal dimensions.

Since the $a$-string $\mathcal{L}_{a}$ (or $\partial \Omega_{a}$ ) does not have any nonreal complex dimension, it is not fractal, in the above sense. This conclusion is entirely compatible with our intuition according to which $\mathcal{L}_{a}$ (or the associated compact set $\partial \Omega_{a} \subseteq \mathbb{R}$ in (2.37)) does not have much complexity.

EXAmPle 2.9. (Self-similar strings.) Next, we discuss a more geometrically interesting family of fractal strings, namely, self-similar fractal strings (as introduced and studied in detail in Lap-vF1-4, see Lap-vF4, Chs. 2 and 3]). It is shown in the just mentioned references that self-similar strings have infinitely many nonreal complex dimensions. In fact, their only real dimension is the Minkowski dimension $D$ (and it is simple); as a result, $D$ is also the maximal real part of the complex dimensions of such strings. Thus, as expected (since their boundaries are nontrival self-similar sets in $\mathbb{R}$ ), self-similar strings are always fractal.

Now, as we may recall from our earlier discussion, there are two kinds of selfsimilar strings, lattice strings and nonlattice strings. For both types of (i.e., for all) self-similar strings, the geometric zeta function admits a meromorphic continuation to all of $\mathbb{C}$. In the lattice case (i.e., when $G=r^{\mathbb{Z}}$, for some $r \in(0,1)$, where $G$ is the multiplicative group generated by the distinct scaling ratios and the 'gaps' of the self-similar string), the complex dimensions are periodically distributed (with the same vertical period $\mathbf{p}:=2 \pi / \log \left(r^{-1}\right)>0$, called the oscillatory period of the given lattice string) along finitely many vertical lines 33 Furthermore, on each of these vertical lines, the multiplicity of the complex dimensions is the same, while the right most vertical line is $\{\operatorname{Re}(s)=D\}$, on which lie the principal complex dimensions (which are necessarily all simple).

By contrast, in the nonlattice case (i.e., when the rank of the group $G$ is strictly greater than 1), the complex dimensions are no longer periodically distributed. In fact, typically, on a given vertical line $\{\operatorname{Re}(s)=\alpha\}$, with $\alpha \in \mathbb{R}$, there is either zero, or one complex dimension, necessarily $D$ itself (only if $\alpha=D$ ), or else two complex conjugate nonreal complex dimensions $\omega, \bar{\omega}$ (with $\operatorname{Re}(\omega)=\alpha<D)$. (Note that there can be at most countably vertical lines containing at least one

\footnotetext{
${ }^{32}$ It is possible that some of the numbers $-n D$, with $n \in \mathbb{N}$, do not truly appear in 2.39), depending on the value of $a$. However, $D$ is always a complex dimension and "typically", we have an equality (rather than an inclusion) in (2.39).

${ }^{33}$ The number of vertical lines (counted according to multiplicity) is equal to the degree of the polynomial obtained after making the change of variable $w:=r^{s}$ in the denominator of the expression for $\zeta_{\mathcal{L}}(s)=\left(\sum_{k=1}^{K} g_{k}^{s}\right) /\left(1-\sum_{j=1}^{J} r_{j}^{s}\right)$, where $\left(r_{j}\right)_{j=1}^{J}$ are the (not necessarily distinct) scaling ratios and $\left(g_{k}\right)_{k=1}^{K}$ are the gaps of $\mathcal{L}$, with $J \geq 2$.
} 
complex dimension.) Furthermore, on the right most vertical line, the only complex dimension is $D$ itself, and it is simple. (Hence, according to a version of Theorem 2.2 obtained in Lap-vF4 §8.4], a nonlattice string is always Minkowski measurable, in contrast to a lattice string, which is never so.)

Moreover, it is shown in Lap-vF4, §3.4] by using Diophantine approximation that any nonlattice string $\mathcal{L}$ can be approximated by a sequence of lattice strings $\left\{\mathcal{L}_{n}\right\}_{n=1}^{\infty}$ with oscillatory periods $\mathbf{p}_{n}$ increasing exponentially fast to $+\infty$, as $n \rightarrow \infty$. Hence, the complex dimensions of $\mathcal{L}$ are themselves approximated by the complex dimensions of $\mathcal{L}_{n}$. As a result, the complex dimensions of a nonlattice string exhibit a quasiperiodic structure. (See ibid for more precision and for many examples of quasiperiodic patterns of complex dimensions of nonlattice strings.)

Finally, we refine (as in LapRaZu1) the notion of fractality by saying that a geometric object is 'fractal in dimension $d$ ', where $d \in \mathbb{R}$, if it has a nonreal complex dimension with real part $d$. Hence, 'fractality' in our sense is equivalent to fractality in dimension $d$, for some $d \in \mathbb{R}$. (Clearly, $d \leq D$.) Also, fractality in dimension $D$ amounts to the existence of nonreal principal complex dimensions. According to Lap-vF4, Thms. 2.16 and 3.6], this is always the case for lattice strings (such as the Cantor string) but is never the case for nonlattice strings.

In light of the above discussion, a lattice string is fractal in dimension $d$ for at least one value but at most finitely many values of $d$, whereas by contrast, nonlattice strings are fractal in dimension $d$ for infinitely (but countably) many values of $d$. In fact, in the generic nonlattice case, a significantly stronger statement is true 34 namely, the countable set of such $d$ 's is dense in a single compact interval of the form $\left[d_{\min }, D\right]$, with $-\infty<d_{\min }<D$. This latter density result was obtained by the authors of MorSepVi1 who thereby proved a conjecture made in the generic nonlattice case in Lap-vF5 (see also Lap-vF3-4) 35

In $\$ 3.6$, we will further discuss and broaden the notion of fractality (and the related notion of hyperfractality), but now by focusing on higher-dimensional examples, such as the devil's staircase (i.e., the Cantor graph), rather than on fractal strings.

REMARK 2.10. The lattice/nonlattice dichotomy arose in probabilistic renewal theory [Fel], where it is usually referred to as the arithmetic/nonarithmetic dichotomy. In fractal geometry, it was used in Lall1-3 in connection with self-similar sets and generalizations thereof, and then, e.g., in Lap2-7, KiLap1, Gat, LeviVa, Lap-vF1-7, [Fr, Sab1-3], HamLap, LapPe2-3, LapPeWi1-2, LapLa-vF2-3, LapLu-vF1-2, KeKom, Kom, MorSep, MorSepVi1-2, DubSep, LapRaZu1-9 and Lap10. The notion of generic nonlattice selfsimilar string (or, more generally, spray or even set) was introduced in Lap-vF3-5.

2.6. Inverse spectral problems and the Riemann hypothesis. In this subsection, we briefly discuss the intimate connection between direct (respectively, inverse) spectral problems for fractal strings and the Riemann zeta function (respectively, the Riemann hypothesis). For a recent survey of this subject, we refer

\footnotetext{
${ }^{34} \mathrm{~A}$ nonlattice string is said to be generic if the group $G$ generated by its $M$ distinct scaling ratios is of rank $M$ and $M \geq 2$. It is nongeneric, otherwise.

${ }^{35}$ In general, in the nongeneric nonlattice case, the set of such $d$ 's should be dense in at most finitely (but at least one, ending at $D$ ) compact and pairwise disjoint intervals; this is noted, by means of examples, independently in Lap-vF4 and in DubSep.
} 
the interested reader to Lap9.

2.6.1. Direct spectral problems for fractal strings. Let $\mathcal{L}=\left(\ell_{j}\right)_{j=1}^{\infty}$ (or any of its geometric realizations $\Omega \subseteq \mathbb{R})$ be a fractal string of dimension $D \in(0,1) 36$ and let $N_{\nu}$ denote the associated spectral counting function of $\mathcal{L}$, as defined in $\$ 2.2$ It turns out that the leading spectral asymptotics of $\mathcal{L}$ are given by the so-called Weyl term, $W$ (named after the well-known $N$-dimensional result in Wey1-2 ${ }^{37}$ Namely,

$$
N_{\nu}(x) \sim W(x) \text { as } x \rightarrow+\infty,
$$

where $W=W(x)$ is the Weyl term given by

$$
W(x):=|\Omega|_{1} x, \text { for } x>0,
$$

with $|\Omega|_{1}=\sum_{j=1}^{\infty} \ell_{j}$ being the total length of the string $\mathcal{L}$ (or the 'volume' of $\Omega$ ).

This is a very special (one-dimensional) case of the spectral asymptotics with error term obtained in Lap1 for fractal drums in $\mathbb{R}^{N}$, with $N \geq 1$ arbitrary. (In fact, $N_{\nu}(x)=W(x)+R(x)$, with $R(x)=O\left(x^{D}\right)$ if $\mathcal{M}^{*}<\infty$ and $R(x)=O\left(x^{D+\varepsilon}\right)$ for any $\varepsilon>0$, otherwise, where $D \in(N-1, N)$ is the (upper) Minkowski dimension of $\Omega$, relative to $\partial \Omega$ or in the terminology of $₫ 3.2$, of the RFD $(\partial \Omega, \Omega)$. Here, the Weyl term $W=W(x)$ is proportional to $|\Omega|_{N} x^{N}$, where $|\Omega|_{N}$ is the $N$-dimensional volume of $\Omega$.) It also follows, for example, from a result of [LapPo2.

The following theorem (joint with C. Pomerance), obtained in LapPo2 (and first announced in LapPo1 ), resolved in the affirmative the one-dimensional case of the modified Weyl-Berry (MWB) conjecture stated in Lap1 for fractal drums 38

We refer to Lap1, Lap3, LapPo2, Lap9 and Lap-vF4, §12.5] for physical and mathematical motivations, as well as for many further references (including [BirSol, BroCar, CouHil, FlLap, Ger, GerScm1-2, Gi], Ho1-3, Ivr1-3, LeviVa, Mel1-2], Met1-2, [Ph], ReSi1-3, See1-3, Lap2-3 and Lap-vF4, App. B], along with those cited in footnote [38), concerning the original conjectures of Weyl Wey1-2 for 'smooth drums', Berry [Berr1-2] for 'fractal drums' and their later modifications and extensions in Lap1-3, in particular.

Theorem 2.11 ([LapPo2] $)$. Let $\mathcal{L}$ be a fractal string which is Minkowski measurable and of Minkowski dimension $D \in(0,1)$. Then, $N_{\nu}$, the spectral counting function of $\mathcal{L}$, admits a monotonic asymptotic second term, proportional to $x^{D}$. More specifically,

$$
N_{\nu}(x)=W(x)-c_{D} \mathcal{M} x^{D}+o\left(x^{D}\right) \quad \text { as } \rightarrow+\infty,
$$

where $W(x)=|\Omega|_{1} x$ is the Weyl term given by (2.41) and $\mathcal{M}$ is the Minkowski content of $\mathcal{L}$. Furthermore, the constant $c_{D}$ is positive, depends only on $D$ and is given explicitly by

$$
c_{D}:=(1-D) 2^{-(1-D)}(-\zeta(D)),
$$

where $\zeta=\zeta(s)$ denotes the Riemann zeta function.

\footnotetext{
${ }^{36}$ Recall that for a fractal string, we always have that $D \in[0,1]$.

${ }^{37}$ Here, $\mathcal{L}$ is viewed as the $\operatorname{RFD}(\partial \Omega, \Omega)$, in the terminology of 3.2 with (upper) Minkowski dimension $D \in(0,1)$.

${ }^{38}$ In higher dimensions, the situation is not as clear cut and the MWB conjecture itself needs to be further modified; see, e.g., [FIVa, LapPo3, MolVai].
} 
Theorem 2.11 relies, in particular, on (the easier direction of) the characterization of Minkowski measurability, given in LapPo2 and recalled in Theorem 2.3. (Namely, $\mathcal{L}$ is Minkowski measurable iff $N_{\mathcal{L}}(x) \sim Q x^{D}$, for some constant $Q \in(0,+\infty)$, where $N_{\mathcal{L}}$ is the geometric counting function of $\mathcal{L}$.) It also makes use of the identity (2.21) connecting $N_{\nu}(x)$ and $N_{\mathcal{L}}(x)$ for any $x>0$, as well as of a direct computation involving the analytic continuation of $\zeta(s)$ to the open right half-plane $\{\operatorname{Re}(s)>0\}$ and hence, to the (open) critical strip $\{0<\operatorname{Re}(s)<1\}$ since $D \in(0,1))$.

REMARK 2.12. (a) (Drums with fractal boundary.) The aforementioned spectral error estimates obtained in Lap1 are valid for Laplacians (or, suitably adapted, for more general elliptic operators with variable, nonsmooth coefficients and of order $2 m$, with $m \in \mathbb{N}$ ) on bounded open sets $\Omega \subseteq \mathbb{R}^{N}$ (where $N \geq 1$ is arbitrary) with (possibly) fractal boundary ("drums with fractal boundary", in the sense of Lap3) and with Dirichlet or Neumann boundary conditions. For the Dirichlet problem, $\Omega$ is allowed to have finite volume (i.e., $|\Omega|_{N}<\infty$ ) rather than to be bounded. Furthermore, for the Neumann problem, one must assume, for example, that $\Omega$ satisfies the extension property for the Sobolev space $H^{m}=W^{m, 2}$ Br, Maz, where $m=1$ in the present case of the Laplacian; see, e.g., Maz, Jon, HajKosTu1-2, Lap1, Vel-San]. For instance, for a simply connected planar domain, $\Omega$ is an extension domain if and only if it is a quasidisk (i.e., $\partial \Omega$ is a quasicircle, $\mathbf{P o m m}$ ); that is, $\Omega$ is the homeomorphic quasiconformal image of the open unit disk in $\mathbb{C} 39$ Quasidisk and quasicircles (as well as John domains) are of frequent use in harmonic analysis, partial differential equations, complex dynamics

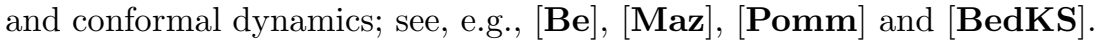

(b) (Drums with fractal membrane.) An analog of the leading term (Weyl's asymptotic formula) and the associated error term in Lap1 discussed in part (a) was obtained in KiLap1 for Laplacians on fractals, rather than on bounded open sets of $\mathbb{R}^{N}$ with fractal boundary; that is, for "drums with fractal membrane" (in the sense of Lap3 ) rather than for "drums with fractal boundary" (as, e.g., in BirSol, BroCar, Lap1-4, LapPo1-3, LapMa1-2, Ger, GerScm1-2, FlLap, FlVa, EdmEv, Dav, LeviVa, HeLap, MolVai, vB-Gi, HamLap, Lap9, LapPa and LapNRG). Examples of physics and mathematics references on Laplacians on fractals include the books $\mathbf{K i}$ and $\mathbf{S t r}$ along with the papers [Ram, RamTo, [Shi], [FukShi], KiLap1-2, Barl], [Ham1-2, Sab1-3], Tep1-2, DerGrVo, Lap5-6], ChrIvLap, CipGIS, LapSar and LalLap1-2, as well as the many relevant references therein.

2.6.2. Inverse spectral problems for fractal strings. Now that we have explicitly and fully solved the above direct spectral problem for fractal strings, it is natural to consider its converse, which is called an inverse spectral problem, (ISP). In fact, we have a one-parameter family of such problems, $(\mathrm{ISP})_{D}$, parametrized by the (Minkowski) dimension $D$. Recall that $D \in(0,1)$ is arbitrary, so that the parameter $D$ sweeps out the entire 'critical interval' $(0,1)$ for the Riemann zeta function $\zeta=\zeta(s)$

${ }^{39}$ Another characterization of a quasidisk is that it is a planar domain which is both a John domain (see John and, e.g., CarlJonYoc, McMul, DieRuzSchu, Dur-LopGar, AcoDur-LopGar or LopGar1-2) and linearly connected; see, e.g., ChuOsgPomm. 
$(\mathrm{ISP})_{D}$ Let $\mathcal{L}$ be a fractal string of Minkowski dimension $D \in(0,1)$ such that its associated spectral counting function $N_{\nu}$ admits a monotonic asymptotic second term, proportional to $x^{D}$. Namely, with the Weyl term $W$ given by (2.41), assume that

$$
N_{\nu}(x)=W(x)-\mathcal{C} x^{D}+o\left(x^{D}\right) \quad \text { as } x \rightarrow+\infty,
$$

for some nonzero constant $\mathcal{C}$ (depending only on $\mathcal{L}$ ). Does it then follow that $\mathcal{L}$ is Minkowski measurable?

The above question à la Marc Kac, $\mathbf{K a c}$, could be coined Can one hear the shape of a fractal string (of dimension $D \in(0,1)$ )? Note, however, that this question (or equivalently, the corresponding inverse spectral problem $\left.(I S P)_{D}\right)$, is of a very different nature from the original one, raised in $\mathbf{K a c}$.

Remark 2.13. Equation (2.44) alone with $\mathcal{C} \neq 0$ and $D \in(0,1)$ implies that $\mathcal{L}$ has Minkowski dimension $D$. Furthermore, if (ISP) $)_{D}$ has an affirmative answer, then it follows from Theorem 2.11 that $\mathcal{C}>0$ and $\mathcal{C}=c_{D} \mathcal{M}$, where $c_{D}>0$ is the constant (depending only on $D$ ) given by (2.43) and $\mathcal{M}$ is the Minkowski content of $\mathcal{L}$.

We can finally give the precise statement of the main result (Theorem 2.14 and Corollary 2.15) connecting the family of inverse spectral problems (ISP) ${ }_{D}$, with $D \in(0,1)$, and the Riemann hypothesis $(\mathrm{RH})$; see LapMa1-2, joint with $\mathrm{H}$. Maier.

Theorem 2.14 (Critical zeros of $\zeta$ and inverse spectral problems; LapMa2 ). Fix $D \in(0,1)$. Then, the inverse spectral problem $(I S P)_{D}$ has an affirmative answer if and only if $\zeta=\zeta(s)$ does not have any zeros on the vertical line $\{\operatorname{Re}(s)=D\}$; i.e., if and only if the 'partial RH' (abbreviated $\left.(R H)_{D}\right)$ holds for this value of $D$. In short (and with the obvious notation), we have that

$$
(R H)_{D} \Leftrightarrow(I S P)_{D}, \text { for any } D \in(0,1) .
$$

COROLlary 2.15 (Spectral reformulation of RH; LapMa2 ). The Riemann hypothesis is true if and only the inverse spectral problem $(I S P)_{D}$ has an affirmative answer for all $D \in(0,1)$, except in the 'midfractal case' when $D=1 / 2$.

Proof. (Proof of Corollary 2.15.) Since it is known that $\zeta$ has a nonreal zero (and even infinitely many zeros, by a theorem of G. H. Hardy; see, e.g., Edw, Tit]) on the critical line $\{\operatorname{Re}(s)=1 / 2\}$, this is a consequence of Theorem 2.14.

REMARK 2.16. Observe that we could reformulate Corollary 2.15 by stating that $\mathrm{RH}$ is equivalent to (ISP) ${ }_{D}$ having an affirmative answer for all $D \in(0,1 / 2)$ (or equivalently, for all $D \in(1 / 2,1))$. This follows from the well-known functional equation for $\zeta$ connecting $\zeta(s)$ and $\zeta(1-s)$, for all $s \in \mathbb{C}$; see, e.g., Edw or Tit.

Proof. (Sketch of the proof of Theorem 2.14.) The proof of one implication in Theorem 2.14 relies on the Wiener-Ikehara Tauberian theorem (see, e.g., $\mathbf{P o s}$ ) or one of its later improvements (see, e.g., PitWie, Pit and Kor).

The converse (i.e., the reverse implication $(I S P)_{D} \Rightarrow(R H)_{D}$ in (2.45) is proved by contraposition. That is, we assume that $(R H)_{D}$ fails and we therefore want to show that the inverse spectral problem $(I S P)_{D}$ does not have an affirmative answer. Hence, suppose that there exists $\omega \in \mathbb{C}$, with $\omega=D+i \tau(D \in(0,1), \tau>0)$, such that $\zeta(\omega)=0$. Then, clearly, $\bar{\omega}=D-i \tau$ also satisfies $\zeta(\bar{\omega})=0$. 
Next, we use the intuition of the notion of complex dimension 40 in order to construct a fractal string $\mathcal{L}=\left(\ell_{j}\right)_{j=1}^{\infty}$ which has oscillations of leading order $x^{D}$ in its geometry but which (because $0=\zeta(\omega)=\overline{\zeta(\omega)}=\zeta(\bar{\omega})$ ) does not have oscillations of order $D$ in its spectrum (so that $N_{\nu}(x)$ has a monotonic asymptotic second term proportional to $x^{D}$ ).

More specifically, we have that (for some positive constant $\beta$ sufficiently small and with $[y]$ denoting the integer part of $y \in \mathbb{R}$ )

$$
N_{\mathcal{L}}(x)=[V(x)] \text {, for any } x>0,
$$

where

$$
\begin{aligned}
V(x) & :=x^{D}+\beta\left(x^{\omega}+x^{\bar{\omega}}\right) \\
& =x^{D}(1+2 \beta \cos (\tau \log x)),
\end{aligned}
$$

for any $x>0$. Note that it suffices to choose a positive number $\beta<1 / 2$ so that $V(x)>0$ for all $x>0$ and $\beta<D / 2(D+\tau)$ so that $V^{\prime}(x)>0$ for all $x>041$ Hence, we can simply choose $\beta \in(0, D / 2(D+\tau))$. Since $V$ is now strictly increasing from $(0,+\infty)$ to itself, we can uniquely define $\ell_{j}>0$ so that $V\left(\ell_{j}^{-1}\right)=j$; i.e., in light of (2.46), $N_{\mathcal{L}}\left(\ell_{j}^{-1}\right)=j$, for each integer $j \geq 1$. This defines the sought for (bounded) fractal string $\mathcal{L}=\left(\ell_{j}\right)_{j=1}^{\infty} \sqrt{42}$ one can then choose any geometric realization $\Omega$ of $\mathcal{L}$ as a subset of $\mathbb{R}$ with finite length.

Since by construction,

$$
N_{\mathcal{L}}(x) \sim x^{D}(1+2 \beta \cos (\tau \log x)) \quad \text { as } x \rightarrow+\infty,
$$

we deduce from (the difficult part of) Theorem 2.3 (the characterization of Minkowski measurability) that the fractal string $\mathcal{L}$ is not Minkowski measurable.

Indeed, because $x^{-D} N_{\mathcal{L}}(x)$ oscillates asymptotically between the positive constants $1-2 \beta$ and $1+2 \beta$ (in light of (2.48) $), x^{-D} N_{\mathcal{L}}(x)$ cannot have a limit as $x \rightarrow+\infty$; equivalently, $j^{1 / D} \ell_{j}$ cannot have a limit as $j \rightarrow \infty$, which violates condition (ii) of Theorem 2.3 and therefore shows that $\mathcal{L}$ is not Minkowski measurable, as desired 43

Next, a direct (but relatively involved) computation in LapMa2 (based, in particular, on the identity (2.22) and several key properties of $\zeta=\zeta(s)$ and its meromorphic continuation to the critical strip $\{0<\operatorname{Re}(s)<1\})$ shows that there exist nonzero constants $E_{D}$ and $E_{\omega}$ such that as $x \rightarrow+\infty$,

$$
N_{\nu}(x)=W(x)+E_{D} \zeta(D) x^{D}+E_{\omega} \zeta(\omega) x^{\omega}+\bar{E}_{\omega} \zeta(\bar{\omega})+o\left(x^{D}\right),
$$

where $W(x)=|\Omega|_{1} x$ is the Weyl term (as given by (2.41)).

\footnotetext{
${ }^{40}$ At the time, in the early 1990s, the rigorous definition of complex dimension did not yet exist, even for fractal strings. This only came later, in the mid-1990s; see Lap-vF1, Lap-vF2.

${ }^{41}$ Indeed, an elementary computation shows that$$
V^{\prime}(x)=x^{D-1}(D+2 \beta D \cos (\omega \log x)-2 \beta \tau \sin (\omega \log x)) \text {, for all } x>0 .
$$

${ }^{42}$ Note that $\mathcal{L}$ is bounded because, in light of (2.48), $N_{\mathcal{L}}(x)$ is of the order of $x^{D}$ as $x \rightarrow+\infty$ and hence, $\ell_{j}$ is of the order of $j^{-1 / D}$ as $j \rightarrow \infty$. Since $D \in(0,1)$, it follows that $\sum_{j \geq 1} \ell_{j}<\infty$.

${ }^{43}$ It follows from another theorem in LapPo2 that $\mathcal{L}$ is Minkowski nondegenerate (i.e., $\left.0<\mathcal{M}_{*}(\leq) \mathcal{M}^{*}<\infty\right)$ and has Minkowski dimension $D$; so that $0<\mathcal{M}_{*}<\mathcal{M}^{*}<\infty$, by combining these two results.
} 
Now, since $\zeta(\omega)=\zeta(\bar{\omega})=0$, we see that $N_{\nu}(x)$ has a monotonic asymptotic second term, proportional to $x^{D}$, as claimed:

$$
N_{\nu}(x)=W(x)+E_{D} \zeta(D) x^{D}+o\left(x^{D}\right) \quad \text { as } x \rightarrow+\infty .
$$

This shows that the fractal string $\mathcal{L}$ which we have constructed is not Minkowski measurable but that its spectral counting function has a monotonic asymptotic second term, of the order of $x^{D}$. Therefore, the inverse spectral problem (ISP) ${ }_{D}$ does not have an affirmative answer for this value of $D \in(0,1)$. Thus the implication $(I S P)_{D} \Rightarrow(R H)_{D}$ is now established. This concludes the sketch of the proof of Theorem 2.14 (and hence also of Corollary 2.15).

Remark 2.17. (a) The proof of Theorem 2.14 given in LapMa2 actually shows that one can replace $o\left(x^{D}\right)$ by $O(1)$ in (2.49), and hence also in (2.50).

(b) In the language of the mathematical theory of complex dimensions, the fractal string $\mathcal{L}$ constructed just above has three (simple) complex dimensions; namely, the Minkowski dimension $D$ and the complex conjugate pair of nonreal (principal) complex dimensions $(\omega, \bar{\omega})=(D+i \tau, D-i \tau)$. Hence,

$$
\mathcal{D}_{\mathcal{L}}=\{D, \omega, \bar{\omega}\} .
$$

Accordingly, $\mathcal{L}$ (or, equivalently, any of its geometric realizations $\Omega$ as an open subset of $\mathbb{R}$ with finite length) is fractal (in the sense of $\$ 2.5$ ) and even 'critically fractal' (since it is fractal in the maximal possible dimension, $D$ ).

(c) Theorem 2.14 (suitably interpreted), along with Corollary 2.15, has been extended to a large class of arithmetic zeta functions (and other Dirichlet series) in Lap-vF2, Lap-vF3, Lap-vF4; see Lap-vF4, Ch. 9]. This broad generalization relies on the mathematical theory of complex dimensions developed in those references, and especially, on the fractal explicit formulas obtained therein (see [Lap-vF4, Chs. 5-6]), of which a few examples were provided in 92.2 .

(d) As was alluded to earlier, the second part of the proof of Theorem 2.14 (namely, the proof of the implication $\left.(I S P)_{D} \Rightarrow(R H)_{D}\right)$ was motivated by the intuition of the notion of complex dimensions. At the same time, it served as a powerful incentive for developing the mathematical theory of complex dimensions. Along with the statements of Theorem 2.14 and Corollary 2.15, it also provided a natural geometric interpretation of the (closed) critical strip $\{0 \leq \operatorname{Re}(s) \leq 1\}$. In particular, the midfractal case when $D=1 / 2$ corresponds to the critical line $\{\operatorname{Re}(s)=1 / 2\}$, while the least (respectively, most) fractal case when $D=0$ (respectively, $D=1$ ) corresponds to the left (respectively, right) most vertical line in the closed critical strip, $\{\operatorname{Re}(s)=0\}$ (respectively, $\{\operatorname{Re}(s)=1\}$ ). This observation has played a key role in later work, including [Lap-vF4, Lap7-9 and HerLap1.

It is also in agreement with the author's conjecture according to which there should exist a natural fractal-like geometry whose complex dimensions coincide with the union of $\{1 / 2\}$ and the critical zeros of $\zeta$. Consequently, it would have Minkowski dimension 1/2 and apart from 1/2, the critical zeros $\rho$ of $\zeta$ would be its principal complex dimensions. It is possible that instead, this "geometry" would only be critically subfractal (in dimension $\frac{1}{2}$ ), with the critical zeros $\rho$ of $\zeta$ being precisely its complex dimensions with real part $1 / 2$ (assuming $\mathrm{RH}$, for clarity). Namely, in that case, it would have Minkowski dimension 1 (corresponding to the only pole of $\zeta$, which is simple), midfractal complex dimensions the critical zeros of $\zeta$, and possibly one other (simple) complex dimension at 0 (provided it has the 
perfect symmetry of the completed Riemann zeta function $\xi$, as described in the next paragraph). Accordingly (and still assuming $\mathrm{RH}$, for clarity), in either the former case or the latter case, this geometry would be fractal in dimension $1 / 2$, but not fractal in any other dimension $d \in \mathbb{R}$, with $d \neq 1 / 2$ (since both 0 and 1 are real).

Hence (in the latter case), in algebraic geometric terms, the set $\mathcal{D}_{\zeta}$ of complex dimensions of this elusive fractal geometry would coincide with the divisor of the completed (or global) Riemann zeta function $\xi(s):=\pi^{-s / 2} \Gamma(s / 2) \zeta(s)$ (or, equivalently, with the poles of minus the logarithmic derivative, $-\xi^{\prime}(s) / \xi(s)$ ), leaving apart the multiplicities, which can be explicitly recovered by considering the corresponding residues. Namely, since the zeros of $\xi$ coincide with the critical zeros of $\zeta$ and $\xi$ satisfies the celebrated functional equation $\xi(s)=\xi(1-s)$ for all $s \in \mathbb{C}$, from which it follows that $\xi$ has a (simple) pole at $s=0$ and $s=1$, we would have

$$
\mathcal{D}_{\zeta}=\{0,1\} \cup\{\text { critical zeros of } \zeta\},
$$

where each complex dimension is counted with multiplicity one. Accordingly, the sought for fractal-like geometry would be "self-dual' (in the sense of [Lap7]), reflecting the perfect symmetry of the functional equation with respect to the critical line $\{\operatorname{Re}(s)=1 / 2\}$. (See also 4.4 and Lap10.)

\section{A Taste of the Higher-Dimensional Theory: Complex Dimensions and Relative Fractal Drums (RFDs)}

In this section, which by necessity of concision, will be significantly shorter than would be warranted, we limit ourselves to giving a brief overview of some of the key definitions and results of the higher-dimensional theory of complex dimensions, with emphasis on several key examples illustrating them. [We refer to Lap10, Ch. 3] for a more extensive overview, and to the book LapRaZu1 along with the accompanying papers LapRaZu2-10 (including the two survey articles LapRaZu8-9]) for a much more detailed account of the theory, with complete proofs.] First, however, we begin by providing a short history of one aspect of the subject.

3.1. Brief history. For a long time, the theory of complex dimensions was restricted to the one-dimensional case (corresponding to fractal strings and arbitrary compact subsets of $\mathbb{R}$ ) or in the higher-dimensional case, to very special (although useful) geometries; namely, to fractal sprays Lap3, LapPo3, obtained as countable disjoint unions of scaled copies of one or finitely many generators, and particularly, to self-similar sprays.

An approximate tube formula was first obtained in Lap-vF2-4 for the devil's staircase (i.e., the graph of the well-known Cantor function), from which the complex dimensions of the Cantor graph could be deduced, by analogy with the fractal tube formula for fractal strings (discussed in \$2.1). Then, for the important example of the snowflake curve (or, equivalently, of the Koch curve), an exact fractal tube formula was obtained by the author and Erin Pearse in LapPe1 via a direct computation, based in part on symmetry considerations 44 (See Lap-vF4, \$12.2.1] for an exposition.) Again, the complex dimensions of the Koch curve could be deduced by analogy with the case of fractal strings. However, no analog of the

\footnotetext{
${ }^{44}$ In that context, an interesting open problem remains to explicitly determine the Fourier coefficients of a nonlinear (and periodic) analog of the Cantor function arising naturally in the computation leading to the corresponding fractal tube formula.
} 
geometric zeta functions of fractal strings was used in the process, and therefore, the complex dimensions of the Koch curve (like those of the Cantor graph at that stage) could not yet be precisely defined.

Another important step was carried out by the author and Erin Pearse in LapPe2-3 and significantly more generally, in joint work of those two authors and Steffen Winter in LapPeWi1, where fractal tube formulas were obtained for a large class of fractal sprays LapPo3, and especially, of self-similar sprays or self-similar tilings (as in [Lap2-3], [Pe, LapPe1-2], PeWi] and LapPeWi1-2]) 45 This time, the resulting fractal tube formulas made use of certain "tubular zeta functions", but those zeta functions were of a rather ad hoc nature and could not be extended to more general types of fractal geometries. Also, of course, fractal sprays are rather special cases of fractals 46

Therefore, there still remained to find appropriate fractal-type zeta functions which enabled one, in particular, to both define the complex dimensions of arbitrary bounded (or, equivalently, compact) subsets of $\mathbb{R}^{N}$ (for any $N \geq 1$ ) and to obtain fractal tube formulas valid in this general higher-dimensional setting.

Finally, in 2009, this significant hurdle was overcome when the author introduced a fractal zeta function, now called the distance zeta function, which could be used to achieve the aforementioned goals. This was only the beginning of what turned out to be a very fruitful and creative period, extending from 2009 through 2017, during which large parts of the higher-dimensional theory of complex dimensions and associated fractal tube formulas were developed by the author, Goran Radunović and Darko Žubrinić in the series of papers LapRaZu2-9 as well as in the nearly 700-page research book LapRaZu1.

Our goal here is not to give a detailed account of the theory. Instead, we simply wish to highlight in the rest of this section a few definitions, results and useful examples. We refer to LapRaZu2-10, Lap10, Ch. 3] and, especially, to the research monograph [LapRaZu1, for a more detailed account, as well as for precise statements (and complete proofs) of the main results.

3.2. Fractal zeta functions and relative fractal drums (FZFs and RFDs). The theory of complex dimensions of LapRaZu1-10 is developed for arbitrary bounded subsets $A$ of $\mathbb{R}^{N}$ and, more generally, for arbitrary relative fractal drums (RFDs) $(A, \Omega)$ in $\mathbb{R}^{N}$, for any integer $N \geq 1$, as we now briefly explain.

Given $A$ a (nonempty) subset of $\mathbb{R}^{N}$ and $\Omega$ a possibly unbounded open subset of $\mathbb{R}^{N}$ with finite volume and such that $\Omega \subseteq A_{\delta_{1}}$, for some $\delta_{1}>0$, the pair $(A, \Omega)$ is called a relative fractal drum (or $R F D$, in short) in $\mathbb{R}^{N}$.

Two important special cases of RFDs are $(i)$ when $N=1, \Omega$ is a bounded open subset of $\mathbb{R}^{N}$, and $A:=\partial \Omega$, and (ii) when $N \geq 1$ is arbitrary, $A$ is any bounded subset of $\mathbb{R}^{N}$, and $\Omega:=A_{\delta_{1}}$, for some fixed (but arbitrary) $\delta_{1}>0$. Case $(i)$ just above corresponds to the (ordinary or bounded) fractal strings discussed in $\$ 2$ (but now viewed as the RFDs $(\partial \Omega, \Omega)$ ), while case (ii) corresponds to arbitrary bounded subsets $A$ of $\mathbb{R}^{N}$ (for any integer $N \geq 1$ ); see also 33.2 .2 or 33.2 .1 , respectively.

It is a simple matter to extend the definition of the Minkowski dimension and of the Minkowski content to RFDs, as we now explain. (In the sequel, given a

\footnotetext{
${ }^{45}$ See [Lap-vF4 \$13.1] for an exposition of part of these results.

${ }^{46}$ In one dimension, however, fractal sprays reduce to fractal strings, while the latter enables us to deal with the general case of arbitrary bounded (or, equivalently, compact) subsets of the real line $\mathbb{R}$.
} 
measurable subset $B$ of $\mathbb{R}^{N}$, we let $|B|_{N}=|B|$ denote the $N$-dimensional volume or Lebesgue measure of $B$.)

Given an $\operatorname{RFD}(A, \Omega)$ in $\mathbb{R}^{N}$, we define its tube function

$$
\varepsilon \mapsto V(\varepsilon)=V_{A, \Omega}(\varepsilon):=\left|A_{\varepsilon} \cap \Omega\right|, \text { for } \varepsilon>0,
$$

and then 47 its upper Minkowski dimension

$$
\begin{aligned}
\bar{D} & =\overline{\operatorname{dim}}_{B}(A, \Omega) \\
& =\inf \left\{\beta \in \mathbb{R}: V(\varepsilon)=O\left(\varepsilon^{N-\beta}\right) \quad \text { as } \varepsilon \rightarrow 0^{+}\right\} \\
& =\inf \left\{\beta \in \mathbb{R}: \limsup _{\varepsilon \rightarrow 0^{+}} \frac{V(\varepsilon)}{\varepsilon^{N-\beta}}<\infty\right\} .
\end{aligned}
$$

We define similarly $\underline{D}=\underline{\operatorname{dim}}_{B}(A, \Omega)$, the lower Minkowski dimension of $(A, \Omega)$, by simply replacing the upper limit by a lower limit on the right-hand side of the last equality of (3.2). In general, we have $\underline{D} \leq \bar{D}$ but if $\underline{D}=\bar{D}$ (which is the case for most classic fractals), we denote by $D$ this common value and call it the Minkowski dimension of $(A, \Omega)$; the latter is then said to exist.

The upper (respectively, lower) Minkowski content of $(A, \Omega)$ is then defined (still with $V(\varepsilon)=V_{A, \Omega}(\varepsilon)$ ) by

$$
\mathcal{M}^{*}=\limsup _{\varepsilon \rightarrow 0^{+}} \frac{V_{A, \Omega}(\varepsilon)}{\varepsilon^{N-D}}
$$

and

$$
\mathcal{M}_{*}=\liminf _{\varepsilon \rightarrow 0^{+}} \frac{V_{A, \Omega}(\varepsilon)}{\varepsilon^{N-D}} .
$$

We clearly have $0 \leq \mathcal{M}_{*} \leq \mathcal{M}^{*} \leq \infty$. If $\mathcal{M}_{*}>0$ and $\mathcal{M}^{*}<\infty$, we say that $(A, \Omega)$ is Minkowski nondegenerate. If, in addition, $\mathcal{M}^{*}=\mathcal{M}_{*}$ (i.e., if the limit in (3.3) and (3.4) exists and is in $(0,+\infty))$, then we denote by $\mathcal{M}$, and call the Minkowski content of $(A, \Omega)$, this common value and say that the $\operatorname{RFD}(A, \Omega)$ is Minkowski measurable. It is easy to check that if $(A, \Omega)$ is Minkowski nondegenerate (and, in particular, if it is Minkowski measurable), then its Minkowski dimension $D$ exists.

We note that the upper Minkowski dimension of an RFD can be negative or even take the value $-\infty$. For instance, let $A:=\{(0,0)\}$ and for $\alpha>1$, let

$$
\Omega:=\left\{(x, y) \in \mathbb{R}^{2}: 0<y<x^{\alpha}, x \in(0,1)\right\} .
$$

Then the $\operatorname{RFD}(A, \Omega)$ in $\mathbb{R}^{2}$ has (relative) Minkowski dimension $D=1-\alpha<0$, which takes arbitrary large negative values as $\alpha \rightarrow+\infty$. Furthermore, $(A, \Omega)$ is Minkowski measurable with (relative) Minkowski content $\mathcal{M}=1 /(1+\alpha)$. (See LapRaZu1, Prop. 4.1.35].)

Further, another RFD $(A, \Omega)$ in $\mathbb{R}^{2}$, defined by $A:=\{(0,0)\}$ and

$$
\Omega:=\left\{(x, y) \in \mathbb{R}^{2}: 0<y<e^{-1 / x}, 0<x<1\right\},
$$

is such that its Minkowski dimension $D$ exists (as in the previous example) but is no longer finite; namely, $D=-\infty$. (See LapRaZu1, Cor. 4.1.38].)

\footnotetext{
${ }^{47}$ If $A$ is a bounded subset of $\mathbb{R}^{N}$ (viewed as an RFD, see 3.2.1), we then simply write $V(\varepsilon)=V_{A}(\varepsilon)$. Note that we then have $V_{A}(\varepsilon)=\left|A_{\varepsilon}\right|$.
} 
For a fractal string or for an arbitrary bounded subset $A$ of $\mathbb{R}^{N}$, however, we always have that $\underline{D} \geq 0$ and hence also, that $\bar{D} \geq 0$ (so that when the Minkowski dimension $D$ exists, then $D \geq 0) 48$

RFDs extend the notion of a bounded set in $\mathbb{R}^{N}$ (see 3.2 .1 below), of a fractal string (when $N=1$, see 3.2 .2 ) and more generally, of a fractal drum $(\partial \Omega, \Omega$ ), with $\Omega$ of finite volume in $\mathbb{R}^{N}$ (used, e.g., in Lap1-3 in the case of Dirichlet boundary conditions). They are also very useful tools in the computation of the fractal zeta functions and complex dimensions of fractals (especially when some kind of self-similarity is present), by means of scaling and symmetry considerations; see LapRaZu1 and LapRaZu4.

REMARK 3.1. (Relative box dimension.) It is natural to wonder if there exists a notion of box dimension which is also valid for RFDs and thus, for which the corresponding values can be negative. It turns out to be the case, as is explained in a work in preparation by the authors of LapRaZu1. This (upper) "relative box dimension" not only exists but also coincides with the (upper) relative Minkowski dimension of the given $\operatorname{RFD}(A, \Omega)$ in $\mathbb{R}^{N}$, as in the usual case of bounded subsets $A$ of $\mathbb{R}^{N}$ (see, e.g., [Fa1]) 49 However, its definition now requires a fractional counting of the boxes involved (corresponding essentially to the relative volume of those boxes).

Furthermore, for RFDs of the form $(\partial \Omega, \Omega)$, with $\Omega$ a John domain in $\mathbb{R}^{N}$ $(N \geq 1) 50$ one can show that as in the usual case of bounded subsets of $\mathbb{R}^{N}$, the relative box dimension of the RFD exists and not only coincides with the relative Minkowski dimension, $\operatorname{dim}_{B}(\partial \Omega, \Omega)$, but is also nonnegative.

We note that this notion of (possibly negative) relative box dimension (and that of relative Minkowski dimension, with which it coincides) could perhaps be used to make sense of the heuristic and elusive notion of "negative dimension" and "degree of emptiness" used (or sought for) in ManFra] see also Tri4], in the context of the Hausdorff (rather than of the Minkowski) dimension.

We now define as follows the distance zeta function of an $\operatorname{RFD}(A, \Omega)$ in $\mathbb{R}^{N}$ :

$$
\zeta_{A, \Omega}(s)=\int_{\Omega} d(x, A)^{s-N} d x
$$

for all $s \in \mathbb{C}$ with $R e(s)$ sufficiently large 51

Another very useful fractal zeta function is the closely related tube zeta function of the $\operatorname{RFD}(A, \Omega)$,

$$
\widetilde{\zeta}_{A, \Omega}(s)=\int_{0}^{\delta} V_{A, \Omega}(\varepsilon) \varepsilon^{s-N} \frac{d \varepsilon}{\varepsilon}
$$

\footnotetext{
${ }^{48}$ To see why this is true in the latter case, simply note that $A \cap A_{\delta}=A$ for every $\delta>0$.

${ }^{49}$ It then follows that in the case of a bounded open set with an external cusp $p$ (such as in (3.5) and (3.6), respectively, and the text surrounding them), the relative box dimension of the $\operatorname{RFD}(\{p\}, \Omega)$ is negative or even equal to $-\infty$.

${ }^{50}$ See footnote 39 and the references therein, including John, CarlJonYoc, McMul and ChuOsgPomm, for example.

${ }^{51}$ More specifically, according to property $(b)$ of 3.3 this means that (3.7) holds for all $s \in \mathbb{C}$ with $\operatorname{Re}(s)>\bar{D}$, where $\bar{D}:=\overline{\operatorname{dim}}_{B}(A, \Omega)$, and that $\bar{D}$ is best possible.
} 
for all $s \in \mathbb{C}$ with $\operatorname{Re}(s)$ sufficiently larg 55 and for $\delta>0$ fixed but arbitrary 53

One can show that we have the following functional equation:

$$
\zeta_{A, A_{\delta} \cap \Omega}(s)=\delta^{s-N}\left|A_{\delta} \cap \Omega\right|+(N-s) \widetilde{\zeta}_{A, \Omega}(s),
$$

from which one deduces that $\zeta_{A, \Omega}$ and $\widetilde{\zeta}_{A, \Omega}$ contain essentially the same information, provided $\bar{D}<N$ and $\delta>055$ Indeed, under this very mild condition, $\zeta_{A, \Omega}$ has a meromorphic extension in a given domain $U \subseteq \mathbb{C}$ if and only if $\widetilde{\zeta}_{A, \Omega}$ does, and in this case, $\zeta_{A, \Omega}$ and $\widetilde{\zeta}_{A, \Omega}$ have the same poles (i.e., the same visible complex dimensions) in $U$, with the same multiplicities. Furthermore, still in this case, if $\omega \in U$ is a simple pole of $\widetilde{\zeta}_{A, \Omega}$, then it is also a simple pole of $\zeta_{A, A_{\delta} \cap \Omega}$ and

$$
\operatorname{res}\left(\widetilde{\zeta}_{A, \Omega}, \omega\right)=\frac{1}{N-\omega} \operatorname{res}\left(\zeta_{A, A_{\delta} \cap \Omega}, \omega\right) .
$$

We stress that since according to the definition of an RFD, $\Omega \subseteq A_{\delta_{1}}$ for some $\delta_{1}>0$, we have $A_{\delta} \cap \Omega=\Omega$ for any $\delta \geq \delta_{1}$. Hence, under that condition, we can replace $\zeta_{A, A_{\delta} \cap \Omega}$ by $\zeta_{A, \Omega}$ in both (3.9) and (3.10), as well as in the above statements. The latter equations (3.9) and (3.10) then become (for every $\delta \geq \delta_{1}$ ), respectively,

$$
\zeta_{A, \Omega}(s)=\delta^{s-N}|\Omega|+(N-s) \widetilde{\zeta}_{A, \Omega}(s)
$$

and

$$
\operatorname{res}\left(\widetilde{\zeta}_{A, \Omega}, \omega\right)=\frac{1}{N-\omega} \operatorname{res}\left(\zeta_{A, \Omega}, \omega\right)
$$

In the sequel, we will assume, most of the time implicitly, that $\delta \geq \delta_{1}$ (and $\bar{D}<N$ ); so that (3.9) can be written in the simpler form of the functional equation (3.11).

Given a domain $U \subseteq \mathbb{C}$ containing the "critical line" $\{\operatorname{Re}(s)=\bar{D}\}$ and assuming that $\zeta_{A, \Omega}$ has a (necessarily unique) meromorphic continuation to $U$, the poles of $\zeta_{A, \Omega}$ are called the visible complex dimensions of the $\operatorname{RFD}(A, \Omega)$. If $U=\mathbb{C}$ (or if there is no ambiguity as to the choice of $U$ ), we simply call them the complex dimensions of $(A, \Omega)$.

The set (really, multiset) of (visible) complex dimensions of $(A, \Omega)$ is denoted by $\mathcal{D}\left(\zeta_{A, \Omega}\right)$ (or $\mathcal{D}\left(\zeta_{A, \Omega} ; U\right)$ if we want to specify $U$ ) and when $U=\mathbb{C}$, we also use the notation $\operatorname{dim}_{\mathbb{C}}(A, \Omega):=\mathcal{D}\left(\zeta_{A, \Omega} ; \mathbb{C}\right)$.

We adopt a similar notation when $\zeta_{A, \Omega}$ is replaced by $\widetilde{\zeta}_{A, \Omega}$. In fact, as we alluded to earlier, we will always assume that $\bar{D}<N$; so that $\mathcal{D}\left(\widetilde{\zeta}_{A, \Omega} ; U\right)=$ $\mathcal{D}\left(\zeta_{A, \Omega} ; U\right)$, in which case we also denote by $\mathcal{D}_{A, \Omega}\left(=\mathcal{D}_{A, \Omega}(U)\right)$ the set (or really, multiset) of (visible) complex dimensions of $(A, \Omega)$.

3.2.1. The special case of bounded sets in $\mathbb{R}^{N}$. If $A$ is a bounded subset of $\mathbb{R}^{N}$, then for any fixed $\delta>0$ and for all $s \in \mathbb{C}$ with $\operatorname{Re}(s)$ large enough (really, for all $s \in \mathbb{C}$ with $\operatorname{Re}(s)>\bar{D})$,

$$
\zeta_{A}(s):=\zeta_{A, A_{\delta}}(s)=\int_{A_{\delta}} d(x, A)^{s-N} d x
$$

\footnotetext{
${ }^{52}$ This can be interpreted exactly as in footnote 51 just above, provided $\bar{D}<N$.

${ }^{53}$ It turns out that the poles of $\widetilde{\zeta}_{A, \Omega}$ (i.e., the complex dimensions of $(A, \Omega)$ ) are independent of the choice of $\delta>0$ since changing the value of $\delta$ in (3.8) amounts to adding an entire function to $\widetilde{\zeta}_{A, \Omega}$.

${ }^{54}$ Note that it follows at once from (3.2) that we always have that $\bar{D} \leq N$, since $|\Omega|<\infty$ according to the definition of an RFD.
} 
and

$$
\widetilde{\zeta}_{A}(s):=\zeta_{A, A_{\delta}}(s)=\int_{0}^{\delta} V_{A}(\varepsilon) \varepsilon^{s-N} \frac{d \varepsilon}{\varepsilon},
$$

where $V_{A}(\varepsilon):=\left|A_{\varepsilon}\right|$ (as mentioned earlier). Then, (3.11) reduces to the following simpler functional equation, valid for any $\delta>0$ :

$$
\zeta_{A}(s)=\delta^{s-N}\left|A_{\delta}\right|+(N-s) \widetilde{\zeta}_{A}(s) .
$$

Furthermore, (3.12) becomes (provided $\bar{D}<N$ and assuming that $\omega \in U$ is a simple pole of $\widetilde{\zeta}_{A}$ and hence also, of $\zeta_{A}$ ):

$$
\operatorname{res}\left(\widetilde{\zeta}_{A}, \omega\right)=\frac{1}{N-\omega} \operatorname{res}\left(\zeta_{A}, \omega\right)
$$

Moreover, still provided $\bar{D}<N, \zeta_{A}$ has a meromorphic continuation to a given domain $U \subseteq \mathbb{C}$ if and only if $\widetilde{\zeta}_{A}$ does. In this case, $\zeta_{A}$ and $\widetilde{\zeta}_{A}$ have the same poles in $U$, called the visible complex dimensions of $A$ and denoted by $\mathcal{D}_{A}(U)=\mathcal{D}\left(\zeta_{A} ; U\right)=$ $\mathcal{D}\left(\widetilde{\zeta}_{A} ; U\right)$ or, when $U=\mathbb{C}$ or when no ambiguity may arise, by $\mathcal{D}_{A}=\operatorname{dim}_{\mathbb{C}} A=$ $\mathcal{D}\left(\zeta_{A}\right)=\mathcal{D}\left(\widetilde{\zeta}_{A}\right)$. Indeed, the complex dimensions of the RFD $\left(A, A_{\delta}\right)$ (i.e., the complex dimensions of $A$ ) can be defined indifferently as the poles of $\zeta_{A}$ or of $\widetilde{\zeta}_{A}$. In addition, they are independent of the choice of $\delta>0$ because changing the value of $\delta$ in (3.13) or (3.14) amounts to adding an entire function to the original distance zeta function $\zeta_{A}$ or tube zeta function $\widetilde{\zeta}_{A}$, respectively.

Finally, we note that since $d(\cdot, A)=d(\cdot, \bar{A})$, we have that $A_{\delta}=(\bar{A})_{\delta}$ and hence, $V_{A}=V_{\bar{A}}$; so that $\zeta_{A}=\zeta_{\bar{A}}$ and $\widetilde{\zeta}_{A}=\widetilde{\zeta}_{\bar{A}}$. As a result, by simply replacing $A$ by its closure $\bar{A}$ in $\mathbb{R}^{N}$, we may as well assume without loss of generality that $A \subseteq \mathbb{R}^{N}$ is compact instead of just being bounded.

3.2.2. The special case of fractal strings. Let $N=1$ and $\Omega$ be a bounded open subset of $\mathbb{R}$ (or more generally, an open subset of finite length in $\mathbb{R}$ ). Then, we view the $\operatorname{RFD}(\partial \Omega, \Omega)$ in $\mathbb{R}$ as a geometric realization of the fractal string $\mathcal{L}=\left(\ell_{j}\right)_{j=1}^{\infty}$, the sequence of lengths of the connected components (i.e., open intervals) of $\Omega$, as in 92 ,

Remarkably, the distance zeta function $\zeta_{\partial \Omega, \Omega}$ of the $\operatorname{RFD}(\partial \Omega, \Omega)$ and the geometric zeta function $\zeta_{\mathcal{L}}$ of the fractal string $\mathcal{L}=\left(\ell_{j}\right)_{j \geq 1}$ (see $₫ 2$, Equation (2.10) are related via the following very simple functional equation:

$$
\zeta_{\partial \Omega, \Omega}(s)=2^{1-s} \frac{\zeta_{\mathcal{L}}(s)}{s},
$$

valid initially within the open half-plane $\{\operatorname{Re}(s)>\bar{D}\}$, where

$$
\bar{D}=\overline{\operatorname{dim}}_{B}(\partial \Omega, \Omega)=\overline{\operatorname{dim}}_{B} \mathcal{L}=D\left(\zeta_{\partial \Omega, \Omega}\right)=D\left(\zeta_{\mathcal{L}}\right)
$$

and then (upon analytic continuation), within any domain $U \subseteq \mathbb{C}$ containing the closed half-plane $\{\operatorname{Re}(s) \geq \bar{D}\}$ to which $\zeta_{\mathcal{L}}$ (and hence also, $\zeta_{\partial \Omega, \Omega}$ ) can be meromorphically continued.

One therefore deduces from (3.17) that the visible poles of $\zeta_{\partial \Omega, \Omega}$ in $U$ (i.e., the visible complex dimensions of the $\operatorname{RFD}(\partial \Omega, \Omega))$ are the same as those of $\zeta_{\mathcal{L}}$, except for the fact that (provided $0 \in U$ ) 0 is always a visible pole of $\zeta_{\partial \Omega, \Omega}$ (or more precisely, has multiplicity $m+1$, where $m$ is possibly equal to zero and is defined as the multiplicity of 0 as a pole of $\zeta_{\mathcal{L}}$ ). Furthermore, $\zeta_{\mathcal{L}}$ has a meromorphic continuation to $U$ if and only if $\zeta_{\partial \Omega, \Omega}$ does, and in this case, the corresponding 
residues (at a simple pole $\omega \in U, \omega \neq 0$, of either $\zeta_{\mathcal{L}}$ or $\zeta_{\partial \Omega, \Omega}$ ) are related by the following identity 55

$$
\operatorname{res}\left(\zeta_{\partial \Omega, \Omega}, \omega\right)=\frac{2^{1-\omega}}{\omega} \operatorname{res}\left(\zeta_{\mathcal{L}}, \omega\right)
$$

If one starts instead with a given (bounded) fractal string $\mathcal{L}=\left(\ell_{j}\right)_{j \geq 1}$ such that $\sum_{j>1} \ell_{j}<\infty$, then all the above facts and identities are independent of the choice of the geometric realization $\Omega$ of $\mathcal{L}$ as a bounded open subset of $\mathbb{R}$ and hence, of the choice of the RFD $(\partial \Omega, \Omega)$ associated with $\mathcal{L}$.

The above simple observations enable one, in particular, to view the theory of complex dimensions and the associated fractal tube formulas developed in LapRaZu1 and LapRaZu4,6 as containing (as a very special case) the corresponding theory of fractal strings (as developed in Lap-vF4). It also enables us to significantly simplify the statement of the fractal tube formulas for fractal string 56 in the case when 0 happens to be a visible pole of $\zeta_{\mathcal{L}}$. Suffices to say here that, as a result, $\zeta_{\partial \Omega, \Omega}$ and therefore $\mathcal{D}_{\partial \Omega, \Omega}=\mathcal{D}\left(\zeta_{\partial \Omega, \Omega}\right)$ should be considered as the fractal zeta function and the complex dimensions of a fractal string $\mathcal{L}$ or $\Omega$ (rather than $\zeta_{\mathcal{L}}$ and $\mathcal{D}_{\mathcal{L}}=\mathcal{D}\left(\zeta_{\mathcal{L}}\right)$, respectively).

It follows from the functional equation (3.17) and the above discussion that $\mathcal{D}_{\mathcal{L}}=\mathcal{D}\left(\zeta_{\mathcal{L}}\right)$ and $\mathcal{D}_{\partial \Omega, \Omega}=\mathcal{D}\left(\zeta_{\partial \Omega, \Omega}\right)$, respectively the set of complex dimensions of the fractal string $\mathcal{L}$ (in the sense of [Lap-vF4 and $\oint_{2}$ above) and the set of complex dimensions of the associated RFD $(\partial \Omega, \Omega)$ (in the sense of LapRaZu1 and of the present $(3.2)$, for any geometric realization $\Omega \subseteq \mathbb{R}$ of $\mathcal{L}$, are connected via the following relation (between multisets):

$$
\mathcal{D}_{\partial \Omega, \Omega}=\mathcal{D}_{\mathcal{L}} \cup\{0\},
$$

in the sense that if 0 is a pole of $\zeta_{\mathcal{L}}$ (i.e., a complex dimension of $\mathcal{L}$, in the sense of 92) of multiplicity $m \geq 0$, then it is also a pole of $\zeta_{\partial \Omega, \Omega}$ (i.e., a complex dimension of the $\operatorname{RFD}(\partial \Omega, \Omega)$, in the sense of 33.2$)$ of multiplicity $m+1$. All the other (i.e., nonzero) complex dimensions of the fractal string $\mathcal{L}$ and of the RFD $(\partial \Omega, \Omega)$ coincide, and have the same multiplicities.

3.3. A few key properties of fractal zeta functions. In this section, we discuss several important properties of the fractal zeta functions (that is, of the distance and tube zeta functions) of relative fractal drums (RFDs) and, in particular, of bounded sets in $\mathbb{R}^{N}$. Throughout, $N \geq 1$ is a fixed (but arbitrary) positive integer. We will stress the case of distance zeta functions since (except for a few small differences) fractal tube zeta functions have entirely analogous properties.

3.3.1. Abscissa of convergence and holomorphicity. Let $(A, \Omega)$ be an RFD in $\mathbb{R}^{N}$ and consider its distance and tube zeta functions, $\zeta_{A, \Omega}$ and $\widetilde{\zeta}_{A, \Omega}$. In order to simplify the exposition, we still assume that $\bar{D}<N 5$

\footnotetext{
${ }^{55}$ For the simplicity of exposition, we assume throughout this discussion in 3.2 .1 that 0 is not a zero of $\zeta_{\mathcal{L}}: \zeta_{\mathcal{L}}(0) \neq 0$. Otherwise, we would have to make use of the notion of a divisor (in essence, the multiset of zeros and poles) of a meromorphic function, to be discussed and used later in 93 and 4

${ }^{56}$ These formulas were briefly discussed in 2.1 and will be revisited in the relevant part of 3.5 .3

${ }^{57}$ We also implicitly assume throughout $\$ 3.3$ (and beyond) that $\delta \geq \delta_{1}$, where $\Omega \subseteq A_{\delta_{1}}$.
} 
(a) (Abscissa of convergence). It is shown in LapRaZu1 (see also LapRaZu2,4) that the abscissa of convergence $\sigma=D\left(\zeta_{A, \Omega}\right)=D\left(\widetilde{\zeta}_{A, \Omega}\right)$ of $\zeta_{A, \Omega}$ and $\widetilde{\zeta}_{A, \Omega}$ is well defined and coincides with the upper Minkowski dimension $\bar{D}=\overline{\operatorname{dim}}_{B}(A, \Omega)$ of $(A, \Omega)$. Namely, the largest right half-plane $\{\operatorname{Re}(s)>\alpha\}$ (with $\alpha \in(-\infty, N])$ in which the Lebesgue integral defining $\zeta_{A, \Omega}(s)$ (respectively, $\widetilde{\zeta}_{A, \Omega}(s)$ ) in (3.7) (respectively, in (3.8)) converges is $\{\operatorname{Re}(s)>\bar{D}\}$, called the half-plane of (absolute) convergence of $\zeta_{A, \Omega}$ (respectively, of $\widetilde{\zeta}_{A, \Omega}$ ); for example,

$$
\begin{aligned}
\sigma: & =\inf \left\{\alpha \in \mathbb{R}: \int_{\Omega} d(x, A)^{\alpha-N} d x<\infty\right\} \\
& =\bar{D}:=\overline{\operatorname{dim}}_{B}(A, \Omega) .
\end{aligned}
$$

A part of the proof of this key fact relies on a suitable extension of a result obtained in [HarvPol] for a completely different purpose (the study of the singularities of the solutions of certain linear partial differential equations).

(b) (Holomorphicity). It is also shown in the aforementioned references that $\zeta_{A, \Omega}$ (and hence, in light of the functional equation (3.11), also $\widetilde{\zeta}_{A, \Omega}$ ) is holomorphic in the open right half-plane $\{\operatorname{Re}(s)>\bar{D}\}$, where $\bar{D}:=\overline{\operatorname{dim}}_{B}(A, \Omega)$, as before.

In addition, under mild assumptions (namely, if $D$, the Minkowski dimension of $(A, \Omega)$, exists and $\left.\mathcal{M}_{*}>0\right)$, then this right half-plane $\{\operatorname{Re}(s)>\bar{D}\}$ is also optimal from the point of view of holomorphicity because one can show that $\zeta_{A, \Omega}(s) \rightarrow$ $+\infty$, as $s \rightarrow \bar{D}$, with $s \in \mathbb{R}$ and $s>\bar{D}$. Therefore, the half-plane of (absolute) convergence and the half-plane of holomorphic continuation coincide and are equal to $\{\operatorname{Re}(s)>D\}$, in this case. One then says that $D$, the (relative) Minkowski dimension of $(A, \Omega)$, also coincides with $D_{h o l}\left(\zeta_{A, \Omega}\right)=D_{h o l}\left(\widetilde{\zeta}_{A, \Omega}\right)$, the common abscissa of holomorphic continuation of $\zeta_{A, \Omega}$ and of $\widetilde{\zeta}_{A, \Omega} 58$

Finally, one can always (complex) differentiate $\zeta_{A, \Omega}$ or $\widetilde{\zeta}_{A, \Omega}$ under the integral sign and as many times as one wants. For example, provided $\operatorname{Re}(s)>\bar{D}$,

$$
\zeta_{A, \Omega}^{\prime}(s)=\int_{\Omega} d(x, A)^{s-N} \log (d(x, A)) d x .
$$

Naturally, all of the above properties (in part $(a)$ or $(b)$ ) hold without change for $\zeta_{A}$ and $\widetilde{\zeta}_{A}$, the distance and tube zeta functions of bounded sets $A$ in $\mathbb{R}^{N}$ (initially defined for $\operatorname{Re}(s)>\bar{D}$ by (3.13) and (3.14), respectively) by considering the associated RFDs $\left(A, A_{\delta}\right)$, for a fixed but arbitrary $\delta>0$.

It is not known whether we can always have an equality in (3.22) or, equivalently, if $D_{h o l}\left(\zeta_{A, \Omega}\right)=\bar{D}$. By contrast, for fractal strings, the analog of this property always holds 59 see Lap-vF4 or LapRaZu1, §2.1.4]. Note that the property stated in part $(a)$ above is the exact counterpart of Theorem 2.1

Since $\zeta_{A, \Omega}$ and $\widetilde{\zeta}_{A, \Omega}$ admit a holomorphic continuation (necessarily unique) to $\{\operatorname{Re}(s)>\bar{D}\}$ ), they cannot have any pole there. Consequently, the set of complex

\footnotetext{
${ }^{58}$ In general, however, we only have that

$$
D_{h o l}\left(\zeta_{A, \Omega}\right)=D_{h o l}\left(\widetilde{\zeta}_{A, \Omega}\right) \leq \bar{D}
$$

${ }^{59}$ More specifically, provided the (bounded) fractal string $\mathcal{L}=\left(\ell_{j}\right)_{j \geq 1}$ is infinite (i.e., has an infinite number of lengths), then $D_{\text {hol }}\left(\zeta_{\mathcal{L}}\right)=D\left(\zeta_{\mathcal{L}}\right)=\bar{D}$, the (upper) Minkowski dimension of $\mathcal{L}$.
} 
dimensions of $(A, \Omega)$ must be contained in $\{\operatorname{Re}(s) \leq \bar{D}\}$. More specifically, if the domain $U \subseteq \mathbb{C}$ contains the vertical line $\{\operatorname{Re}(s)=\bar{D}\}$ (or, equivalently, contains the closed half-plane $\{\operatorname{Re}(s) \geq \bar{D}\}) 60$ then $\zeta_{A, \Omega}$ (and hence also, $\widetilde{\zeta}_{A, \Omega}$ ) can be meromorphically continued to a domain $U \subseteq \mathbb{C}$, and

$$
\mathcal{D}\left(\zeta_{A, \Omega} ; U\right)=\mathcal{D}\left(\widetilde{\zeta}_{A, \Omega} ; U\right) \subseteq\{\operatorname{Re}(s) \leq \bar{D}\}
$$

In addition, if $\bar{D} \in U$, which is certainly the case if $U$ also contains the vertical line $\{\operatorname{Re}(s)=\bar{D}\}$, then

$$
\bar{D}:=\overline{\operatorname{dim}}_{B}(A, \Omega)=\max \left\{\operatorname{Re}(\omega): \omega \in \mathcal{D}\left(\zeta_{A, \Omega} ; U\right\} .\right.
$$

In other words, the (upper) Minkowski dimension of $(A, \Omega)$ is equal to the maximal real part of the (visible) complex dimensions of $(A, \Omega)$.

In the literature on this topic (see LapRaZu1-10]), the vertical line $\{\operatorname{Re}(s)=$ $\bar{D}\}$ is often called the "critical line" (by analogy with the terminology adopted for the Riemann zeta function, but clearly, with a different meaning). Assume that $\zeta_{A, \Omega}$ (or, equivalently, $\widetilde{\zeta}_{A, \Omega}$ ) can be meromorphically extended (necessarily uniquely) to a domain $U$ containing the critical line $\{\operatorname{Re}(s)=\bar{D}\}$, then

$$
\begin{aligned}
\operatorname{dim}_{P C}(A, \Omega)=\mathcal{D}_{P C}(A, \Omega) & =\mathcal{D}_{P C}\left(\zeta_{A, \Omega}\right)\left(=\mathcal{D}_{P C}\left(\widetilde{\zeta}_{A, \Omega}\right)\right) \\
& :=\left\{\omega \in \mathcal{D}\left(\zeta_{A, \Omega} ; U\right): \operatorname{Re}(\omega)=\bar{D}\right\}
\end{aligned}
$$

is called the set (really, multiset) of principal complex dimensions of the $\operatorname{RFD}(A, \Omega)$. Clearly, $\mathcal{D}_{P C}(A, \Omega)=\mathcal{D}_{P C}\left(\zeta_{A, \Omega}\right)=\mathcal{D}_{P C}\left(\widetilde{\zeta}_{A, \Omega}\right)$ is independent of the choice of the domain $U$ satisfying the above assumption. (An entirely analogous notation and terminology is used for a bounded set $A$ in $\mathbb{R}^{N}$; namely, $\operatorname{dim}_{P C}(A)=\mathcal{D}_{P C}(A)=$ $\mathcal{D}_{P C}\left(\zeta_{A}\right)=\mathcal{D}_{P C}\left(\widetilde{\zeta}_{A}\right)$ denotes the set of principal complex dimensions of $A$.)

3.3.2. Meromorphic continuation and Minkowski content. In this subsection, we state a few results concerning the existence of a meromorphic continuation in a suitable region of the distance or tube zeta function of an $\operatorname{RFD}(A, \Omega)$, along with related results concerning the (upper, lower) Minkowski content of $(A, \Omega)$. We will consider both the Minkowski measurable case and the (log-periodic) Minkowski nonmeasurable case, for which the residue evaluated at $D$ (the Minkowski dimension of $(A, \Omega))$ of the given fractal zeta function is directly connected to the Minkowski content or, respectively, the average Minkowski content of $(A, \Omega)$. For complete proofs of those results and of related results about the existence of a meromorphic extension for various classes of RFDs (or of bounded sets) in $\mathbb{R}^{N}$, we refer to $\$ 2.2$ \$2.3, §3.5, \$3.6 and $\S 4.5$ of LapRaZu1, as well as to LapRaZu3-4.

We shall state the results for RFDs in $\mathbb{R}^{N}$; they, of course, specialize to the case of bounded sets in $\mathbb{R}^{N}$, as explained in $₫ 3.2 .1$. (The proofs are the same in the special case of bounded sets as in the general situation of RFDs.) Throughout, as in 3.2. $(A, \Omega)$ is an RFD such that $\bar{D}<N$, even though this is only needed to easily

\footnotetext{
${ }^{60}$ Caution: There exist RFDs for which such domains $U$ do not exist; in fact, in LapRaZu1. $\S 4.6]$, one constructs RFDs in $\mathbb{R}^{N}$ (and also compact sets in $\mathbb{R}^{N}$, along with fractal strings) such that every point of the vertical line $\{\operatorname{Re}(s)=\bar{D}\}$ is a (nonremovable) singularity of $\zeta_{A, \Omega}$ and of $\widetilde{\zeta}_{A, \Omega}$.
} 
state simultaneously the results both for the distance and the tube zeta functions 61

(a) (Minkowski content and residue). We begin by stating a simple result according to which, if $\zeta_{A, \Omega}$ (and hence also, $\widetilde{\zeta}_{A, \Omega}$, in light of (3.11) ) has a meromorphic extension to a connected open neighborhood of $D$ (where the Minkowski dimension $D=D_{(A, \Omega)}$ of $(A, \Omega)$ is assumed to exist), and if $\mathcal{M}^{*}<\infty$, then 62

$$
(N-D) \mathcal{M}_{*} \leq \operatorname{res}\left(\zeta_{A, \Omega}, D\right) \leq(N-D) \mathcal{M}^{*},
$$

$s=D$ is a simple pole of $\zeta_{A, \Omega}$ (and thus also of $\widetilde{\zeta}_{A, \Omega}$ ) and 63

$$
\mathcal{M}_{*} \leq \operatorname{res}\left(\widetilde{\zeta}_{A, \Omega}, D\right) \leq \mathcal{M}^{*} .
$$

In particular, if $(A, \Omega)$ is assumed to be Minkowski measurable, then

$$
\operatorname{res}\left(\zeta_{A, \Omega}, D\right)=(N-D) \mathcal{M}
$$

and

the Minkowski content of $(A, \Omega)$.

$$
\operatorname{res}\left(\widetilde{\zeta}_{A, \Omega}, D\right)=\mathcal{M}
$$

EXAMPLE 3.2. (Cantor sets.) As an illustration of the above result (as well as of the result stated in part $(c)$ below), we consider the generalized Cantor set $A=C^{(a)} \subseteq[0,1]$, defined much like the usual ternary Cantor $C=C^{(1 / 3)}$, where the parameter $a$ lies in $(0,1 / 2)$. Then, $D$ exists and $A$ is Minkowski nondegenerate but is not Minkowski measurable. More precisely, $D=\operatorname{dim}_{B} A=\log _{a^{-1}} 2$. Furthermore,

$$
\mathcal{M}_{*}=\frac{1}{D}\left(\frac{2 D}{1-D}\right)^{1-D}, \quad \mathcal{M}^{*}=2(1-a)\left(\frac{1}{2}-a\right)^{D-1}
$$

and

$$
\operatorname{res}\left(\widetilde{\zeta}_{A}, D\right)=\frac{2}{\log 2}\left(\frac{1}{2}-a\right)^{D} .
$$

Moreover, we have strict inequalities in (3.28) and (3.27) for this example:

$$
0<\mathcal{M}_{*}<\operatorname{res}\left(\widetilde{\zeta}_{A, D}\right)<\mathcal{M}^{*}<\infty
$$

and analogously for $\operatorname{res}\left(\zeta_{A}, D\right)$. Also, since $D<1$, we have that

$$
\operatorname{res}\left(\zeta_{A}, D\right)=(1-D) \operatorname{res}\left(\widetilde{\zeta}_{A}, D\right),
$$

with $\operatorname{res}\left(\widetilde{\zeta}_{A}, D\right)$ given by (3.32).

We could discuss analogously the examples of the Sierpinski gasket and the Sierpinski carpet. In particular, we would find that the inequalities in (3.27) and (3.28) are also strict in those two cases, because the classic Sierpinski gasket and carpet are both Minkowski nondegenerate and not Minkowski measurable, as can be checked via a direct computation.

\footnotetext{
${ }^{61}$ We also suppose, as before, that $\delta>0$ is such that $\delta \geq \delta_{1}$, where $\Omega \subseteq A_{\delta_{1}}$, which can always be assumed without loss of generality.

${ }^{62}$ Clearly, if $\mathcal{M}_{*}=0$ or $\mathcal{M}^{*}=+\infty$, then the corresponding inequality in (3.27) or in (3.28) is trivial. Hence, we may as well assume that $(A, \Omega)$ is Minkowski nondegenerate in order to obtain the full strength of the result.

${ }^{63}$ The value of the residue $\operatorname{res}\left(\widetilde{\zeta}_{A, \Omega}, D\right)$ is independent of the choice of $\delta>0$ in the definition (3.8) of $\widetilde{\zeta}_{A, \Omega}$, as well as of the choice of $\delta_{1}$ implicit in (3.7) and 3.8. And likewise for the values of the residues $\operatorname{res}\left(\zeta_{A}, D\right)$ and $\operatorname{res}\left(\tilde{\zeta}_{A}, D\right)$ in the definition of $\zeta_{A}$ and $\tilde{\zeta}_{A}$ in (3.13) and (3.14), in the counterpart of this result for a bounded subset $A$ of $\mathbb{R}^{N}$; recall from (3.13) that $\zeta_{A}:=\zeta_{A}, A_{\delta}$.
} 
EXAMPLE 3.3. (a-string.) Another simple illustration of the above result stated in part $(a)$ (as well as of the result stated in part (b) below) is the $a$-string discussed in $\$ 2$ (see Example 2.8 in $\$ 2.5$ ). Recall that for any $a>0$,

$$
\Omega=\Omega_{a}=\bigcup_{j=1}^{\infty}\left((j+1)^{-a}, j^{-a}\right)
$$

and so (with $\left.\partial \Omega_{a}:=\partial\left(\Omega_{a}\right)\right)$

$$
\partial \Omega_{a}=\left\{j^{-a}: j \geq 1\right\} \cup\{0\}
$$

and $\mathcal{L}_{a}=\left(\ell_{j}\right)_{j \geq 1}$, with $\ell_{j}:=j^{-a}-(j+1)^{-a}$ for each $j \geq 1$. Furthermore, the $\operatorname{RFD}\left(\partial \Omega_{a}, \Omega_{a}\right)$ (or, equivalently, the fractal string $\left.\mathcal{L}_{a}\right)$ is Minkowski measurable with Minkowski content

where

$$
\mathcal{M}=\frac{2^{1-D} a^{D}}{1-D}
$$

$$
D=\operatorname{dim}_{B}\left(\partial \Omega_{a}, \Omega_{a}\right)=D_{\mathcal{L}_{a}}=\frac{1}{a+1} .
$$

Moreover, it follows from a direct computation, left as an exercise for the interested reader, that

$$
\operatorname{res}\left(\zeta_{\mathcal{L}_{a}}, D\right)=D a^{D}
$$

which since (in light of (3.19)

$$
\operatorname{res}\left(\zeta_{\partial \Omega_{a}, \Omega_{a}}, D\right)=\frac{2^{1-D}}{D} \operatorname{res}\left(\zeta_{\mathcal{L}_{a}}, D\right)
$$

is in agreement with the exact counterpart for RFDs of (3.34); namely,

$$
\operatorname{res}\left(\zeta_{\partial \Omega_{a}, \Omega_{a}}\right)=(1-D) \operatorname{res}\left(\widetilde{\zeta}_{\partial \Omega_{a}, \Omega_{a}}, D\right) .
$$

Indeed, by combining (3.37)-(3.40), we obtain that

$$
\begin{aligned}
(1-D) \mathcal{M} & =2^{1-D} a^{D}=\frac{2^{1-D}}{D}\left(D a^{D}\right) \\
& =\frac{2^{1-D}}{D} \operatorname{res}\left(\zeta_{\mathcal{L}_{a}}, D\right)=\operatorname{res}\left(\zeta_{\partial \Omega_{a}, \Omega_{a}}, D\right),
\end{aligned}
$$

as desired.

(b) (Existence of a meromorphic extension: Minkowski measurable case). Let $(A, \Omega)$ be an RFD in $\mathbb{R}^{N}$ such that there exists $\alpha>0, \mathcal{M} \in(0,+\infty)$ and $D \geq 0$ such that

$$
V_{A, \Omega}(\varepsilon):=\left|A_{\varepsilon} \cap \Omega\right|=\varepsilon^{N-D}\left(\mathcal{M}+O\left(\varepsilon^{\alpha}\right)\right) \quad \text { as } \varepsilon \rightarrow 0^{+} .
$$

Then, $\operatorname{dim}_{B}(A, \Omega)$ exists and $\operatorname{dim}_{B}(A, \Omega)=D$. Furthermore, $(A, \Omega)$ is Minkowski measurable with Minkowski content equal to $\mathcal{M}$. Moreover, the distance zeta function $\zeta_{A, \Omega}$ has for abscissa of convergence $D$ and possesses a (necessarily unique) meromorphic continuation (still denoted by $\zeta_{A, \Omega}$, as usual) to (at least) the open right half-plane $\{\operatorname{Re}(s)>D-\alpha\}$; that is, $D_{\text {mer }}\left(\zeta_{A, \Omega}\right) \leq D-\alpha$, where $D_{\text {mer }}\left(\zeta_{A, \Omega}\right)$ is the abscissa of meromorphic continuation of $\zeta_{A, \Omega}$ (defined much as the abscissa of holomorphic continuation, except for the adjective "holomorphic" replaced by "meromorphic"). The only pole of $\zeta_{A, \Omega}$ (i.e., the only visible complex dimension of $(A, \Omega))$ in this half-plane is $s=D$; it is simple and $\operatorname{res}\left(\zeta_{A, \Omega}, D\right)=(N-D) \mathcal{M}$. 
Clearly, the same result holds for $\widetilde{\zeta}_{A, \Omega}$, the tube (instead of the distance) zeta function of $(A, \Omega)$, except for the fact that in that case, $\operatorname{res}\left(\widetilde{\zeta}_{A, \Omega}, D\right)=\mathcal{M}$.

EXERCiSE 3.4. (i) Show that the hypotheses of part $(b)$ are satisfied for the $a$-string of Example 3.3 , viewed as the RFD $\left(\partial \Omega_{a}, \Omega_{a}\right)$.

(ii) Prove via a direct computation that $\zeta_{\mathcal{L}_{a}}$ has a meromorphic continuation to all of $\mathbb{C}$ with (simple) poles at $s=D=1 /(a+1)$ and (at possibly a subset of) $\{-D,-2 D, \cdots,-n D, \cdots: n \geq 1\}$. (For a complete answer, see Lap-vF4. Thm. 6.21 and its proof].) Deduce that

$$
\mathcal{D}_{\mathcal{L}_{a}}=\mathcal{D}\left(\zeta_{\partial \Omega_{a}, \Omega_{a}}\right) \subseteq\{D, 0,-D,-2 D, \cdots,-n D, \cdots: n \geq 1\},
$$

where all the complex dimensions are simple and $D$ and 0 are always complex dimensions of the fractal string $\mathcal{L}_{a}$ or equivalently, of the $\operatorname{RFD}\left(\partial \Omega_{a}, \Omega_{a}\right)$.

(c) (Existence of a meromorphic extension: Minkowski nonmeasurable case). Let $(A, \Omega)$ be an RFD in $\mathbb{R}^{N}$ such that there exists $\alpha>0, D \in \mathbb{R}$ and a nonconstant periodic function $G$ with minimal period $T>0$, satisfying

$$
V_{A, \Omega}(\varepsilon):=\left|A_{\varepsilon} \cap \Omega\right|=\varepsilon^{N-D}\left(G\left(\log \varepsilon^{-1}\right)+O\left(\varepsilon^{\alpha}\right)\right) \quad \text { as } \varepsilon \rightarrow 0^{+} .
$$

Then, $\operatorname{dim}_{B}(A, \Omega)$ exists and $\operatorname{dim}_{B}(A, \Omega)=D$. Furthermore, $G$ is continuous and $A$ is Minkowski nondegenerate with lower and upper Minkowski contents respectively given by

$$
\mathcal{M}_{*}=\min G \text { and } \mathcal{M}^{*}=\max G .
$$

Moreover, the tube zeta function $\widetilde{\zeta}_{A, \Omega}$ also has for abscissa of convergence $D\left(\widetilde{\zeta}_{A, \Omega}\right)=$ $D$ and possesses a (necessarily unique) meromorphic extension (still denoted by $\left.\widetilde{\zeta}_{A, \Omega}\right)$ to (at least) the open right half-plane $\{\operatorname{Re}(s)>D-\alpha\}$; that is, $D_{\text {mer }}\left(\widetilde{\zeta}_{A, \Omega}\right) \leq$ $D-\alpha 64$

In addition, the set of all the poles of $\widetilde{\zeta}_{A, \Omega}$ (i.e., the set of visible complex dimensions of the RFD $(A, \Omega))$ in this half-plane is given by

$$
\mathcal{D}_{P C}(A, \Omega)=\operatorname{dim}_{P C}\left(\widetilde{\zeta}_{A, \Omega}\right)=\mathcal{D}\left(\widetilde{\zeta}_{A, \Omega}\right)=\left\{s_{k}:=D+i \frac{2 \pi}{T} k: \widehat{G}\left(\frac{k}{T}\right) \neq 0, k \in \mathbb{Z}\right\},
$$

where

$$
\widehat{G}_{0}(t):=\int_{0}^{T} e^{-2 \pi i t \tau} G(\tau) d \tau, \text { for all } t \in \mathbb{R} .
$$

We have, for every $k \in \mathbb{Z}$,

$$
\operatorname{res}\left(\widetilde{\zeta}_{A, \Omega}, s_{k}\right)=\frac{1}{T} \widehat{G}_{0}\left(\frac{k}{T}\right)
$$

and hence,

$$
\left|\operatorname{res}\left(\widetilde{\zeta}_{A, \Omega}, s_{k}\right)\right| \leq \frac{1}{T} \int_{0}^{T} G(\tau) d \tau
$$

Also,

$$
\lim _{k \rightarrow \pm \infty} \operatorname{res}\left(\widetilde{\zeta}_{A, \Omega}, s_{k}\right)=0
$$

\footnotetext{
${ }^{64}$ We first state the results for $\widetilde{\zeta}_{A, \Omega}$ because they are more elegantly written in this situation; we will then mention the few changes needed for the corresponding statements about $\zeta_{A, \Omega}$.
} 
Finally, the residue of $\widetilde{\zeta}_{A, \Omega}$ at $s=D$ coincides both with the mean value of $G$ and with $\widetilde{\mathcal{M}}$, the average Minkowski content of $(A, \Omega) 65$

$$
\operatorname{res}\left(\widetilde{\zeta}_{A, \Omega}, D\right)=\frac{1}{T} \int_{0}^{T} G(\tau) d \tau=\widetilde{\mathcal{M}}
$$

In particular, the $\operatorname{RFD}(A, \Omega)$ is Minkowski nondegenerate but is not Minkowski measurable and, in fact, we have that

$$
\mathcal{M}_{*}<\operatorname{res}\left(\widetilde{\zeta}_{A, \Omega}, D\right)<\mathcal{M}^{*}<\infty
$$

For the distance (instead of the tube) zeta function $\zeta_{A, \Omega}$, entirely analogous results hold, except for the fact that in light of (3.16),

$$
\operatorname{res}\left(\zeta_{A, \Omega}, s_{k}\right)=\left(N-s_{k}\right) \frac{1}{T} \widehat{G}_{0}\left(\frac{k}{T}\right) \text {, for all } k \in \mathbb{Z},
$$

and hence,

$$
\operatorname{res}\left(\zeta_{A, \Omega}, s_{k}\right)=o(|k|) \quad \text { as }|k| \rightarrow \infty .
$$

ExERCISE 3.5. For the generalized Cantor sets $A=C^{(a)}$ of Example 3.2 above, verify that both the hypotheses and the conclusions (of the counterpart for bounded sets) of part $(c)$ just above are satisfied for an arbitrary $\alpha>0$. Deduce that for any $a \in(0,1 / 2)$, both $\zeta_{A}$ and $\widetilde{\zeta}_{A}$ have a meromorphic extension to all of $\mathbb{C}$ and

$$
\mathcal{D}_{A}=\mathcal{D}\left(\zeta_{A}\right)=\mathcal{D}\left(\widetilde{\zeta}_{A}\right)=\{D+i k \mathbf{p}: k \in \mathbb{Z}\},
$$

where $D:=\log 2 / \log (1 / a)$ and $\mathbf{p}:=2 \pi / \log (1 / a)$ are respectively the Minkowski dimension and the oscillatory period of $A=C^{(a)}$. Also, calculate the average Minkowski content $\widetilde{\mathcal{M}}$ of $A$ both via a direct computation and by using one of the main results of part $(c)$.

The next "exercise" is significantly more difficult than the previous one 66

ExERCISE 3.6. Let $A$ be a self-similar set satisfying the open set condition. Find geometric conditions on $A$ so that in the lattice case (respectively, in the nonlattice case), the hypotheses and hence also the conclusions of the main result (of the counterpart for bounded sets) of part $(c)$ (respectively, part $(b)$ ) of this subsection (i.e., \$3.3.2) are satisfied 67

A variety of significantly more complicated behaviors for the asymptotics (as $\left.\varepsilon \rightarrow 0^{+}\right)$of the tube function $\varepsilon \mapsto\left|A_{\varepsilon} \cap \Omega\right|=V_{A, \Omega}(\varepsilon)$ of an RFD are considered in LapRaZu1. These include, most notably, transcendentally $n$-quasiperiodic behavior, for any $n \in \mathbb{N} \cup\{\infty\}$; see LapRaZu1, §3.1 and §4.6]. Instead of giving the precise (but somewhat involved) definitions and results here, we limit ourselves for now to the following simple example. (Further information will be provided later in the paper, especially in 3.6 ,

${ }^{65}$ Here, $\widetilde{\mathcal{M}}$ is defined as the limit of a suitable Cesaro logarithmic average of $V_{A, \Omega}(t) / t^{N-D}$, much as in Lap-vF4. Thm. 8.30] where $N=1$. More specifically,

$$
\widetilde{\mathcal{M}}:=\lim _{\tau \rightarrow+\infty} \frac{1}{\log \tau} \int_{1 / \tau}^{1} \frac{V_{A, \Omega}(t)}{t^{N-D}} \frac{d t}{t},
$$

where the indicated limit is assumed to exist in $(0,+\infty)$.

${ }^{66}$ In fact, it essentially corresponds to Problem 6.2.35 in LapRaZu1 and would also help solve one part of the much broader Problem 6.2.36 in LapRaZu1.

${ }^{67}$ Recall that a self-similar set is said to be lattice if the multiplicative group generated by its distinct scaling ratios is of rank 1 , and is called nonlattice otherwise. 
Example 3.7. Suppose that the tube function of the bounded set $A$ satisfies (3.43), where the function $G$ is no longer assumed to be periodic (of period $T$ ) but is given instead by $G=G_{1}+G_{2}$, where the nonconstant functions $G_{1}$ and $G_{2}$ are periodic of (minimal) periods $T_{1}$ and $T_{2}$, respectively, with $T_{1} / T_{2}$ irrational. Then, $\zeta_{A}$ has a meromorphic continuation to (at least) $\{\operatorname{Re}(s)>D-\alpha\}$ and the set of principal complex dimensions of $A$ consists of simple (nonremovable) singularities of $\zeta_{A}$ (and of $\widetilde{\zeta}_{A}$ ) and is given by

$$
\mathcal{D}_{P C}(A)=\operatorname{dim}_{P C} A=\bigcup_{j=1}^{2}\left(D+i \frac{2 \pi}{T_{j}} \mathbb{Z}\right)=D+i\left(\bigcup_{j=1}^{2} \frac{2 \pi}{T_{j}} \mathbb{Z}\right),
$$

where $D=D\left(\zeta_{A}\right)=D\left(\widetilde{\zeta}_{A}\right)=\operatorname{dim}_{B} A$. We note that since $T_{1}$ and $T_{2}$ are incommensurable, the imaginary parts of the principal complex dimensions of $A$ have a rather different structure than in part $(c)$ above. In particular, they are no longer in arithmetic progression.

Finally, we point out that the assumptions of this example are realized by the compact subset of $\mathbb{R}$ obtained by taking the disjoint union of two distinct and suitably chosen (two-parameter) generalized Cantor sets; see LapRaZu1, Thm. 3.1.12] for the details and LapRaZu1, Thm. 3.1.15] for the generalization to $n$ such Cantor sets, corresponding to the case when $A$ is (transcendentally) 2quasiperiodic or more generally, $n$-quasiperiodic, respectively. The important (and highly nontrivial) extension to the case when $n=\infty$ is dealt with in LapRaZu1, $\S 4.6]$.

3.3.3. Scaling property and invariance under isometries. We first state the scaling invariance property of the distance zeta function $\zeta_{A, \Omega}$ of an $\operatorname{RFD}(A, \Omega) 68$ (We leave it as an exercise for the interested reader to state its counterpart for the tube zeta function $\widetilde{\zeta}_{A, \Omega}$; alternatively, see [LapRaZu1, §4.1.3].)

For any $\lambda>0$, we have $D\left(\zeta_{\lambda A, \lambda \Omega}\right)=D\left(\zeta_{A, \Omega}\right)=\bar{D}:=\overline{\operatorname{dim}}_{B}(A, \Omega)$ and

$$
\zeta_{\lambda A, \lambda \Omega}(s)=\lambda^{s} \zeta_{A, \Omega}(s)
$$

for all $s \in \mathbb{C}$ with $\operatorname{Re}(s)>\overline{\operatorname{dim}}_{B} A$ or, more generally, for all $s \in U$, where $U$ is any domain containing the closed right half-plane $\{\operatorname{Re}(s) \geq \bar{D}\}$ to which one, and hence both, of these fractal zeta functions has a meromorphic continuation.

Furthermore, if $\omega \in \mathbb{C}$ is a simple pole of the meromorphic extension of $\zeta_{A, \Omega}$ to some open connected neighborhood of the critical line $\{\operatorname{Re}(s)=\bar{D}\}$ (or, equivalently, of $\{\operatorname{Re}(s) \geq \bar{D}\})$, then

$$
\operatorname{res}\left(\zeta_{\lambda A, \lambda \Omega}, \omega\right)=\lambda^{\omega} \operatorname{res}\left(\zeta_{A, \Omega}, \omega\right) .
$$

It is noteworthy that the scaling property of the residues of $\zeta_{A, \Omega}$, as stated in (3.57), is very analogous to the scaling property of Hausdorff measure; the latter, however, is restricted to a single exponent, namely, the Hausdorff dimension, whereas (3.57) is valid for any (visible) complex dimension of $(A, \Omega)$. We do not wish to elaborate on this point here but simply mention that under appropriate hypotheses, a suitable version of these residues should give rise to a family of complex measures, defined by the maps $\Omega \mapsto \operatorname{res}\left(\zeta_{A, \Omega}, \omega\right)$ and indexed by the (visible) complex dimensions $\omega$

\footnotetext{
${ }^{68}$ The properties stated for RFDs in 3.3 .3 and in fact, in all of $33.3 .1-33.3 .4$ have natural counterparts for bounded sets $A$ in $\mathbb{R}^{N}$. In particular, recall from (3.21) and the discussion surrounding it that $D\left(\zeta_{A, A_{\delta}}\right)$ denotes the abscissa of convergence of the RFD $\left(A, A_{\delta}\right)$.
} 
of the bounded set $A$ and where $\Omega$ is allowed to run through the Borel (and not necessarily open) subsets of $\mathbb{R}^{N}$. For more information, see [LapRaZu1, App. B].

Next, we simply mention that the distance and tube zeta functions of an RFD $(A, \Omega)$ are clearly invariant under the group of displacements of $\mathbb{R}^{N}$ (that is, under the group of isometries of the affine space $\mathbb{R}^{N}$, generated by the rotations and translations). More specifically, if $f$ is such a displacement of $\mathbb{R}^{N}$, then

$$
\zeta_{f(A), f(\Omega)}=\zeta_{A, \Omega}
$$

(and analogously for $\widetilde{\zeta}_{A, \Omega}$, as well as for the corresponding upper, lower Minkowski dimensions and contents, and the visible complex dimensions, in particular).

The scaling and invariance properties stated in the present subsection (i.e., \$3.3.3), along with other "covariance" properties of the fractal zeta functions, are very useful in the concrete computation of the distance and tube zeta functions of a variety of examples (including many of those discussed in $\$ 3.4$ ), as well as of the corresponding complex dimensions. They also play an important role in the direct computation of fractal tube formulas for many concrete examples (including several of those discussed in \$3.5.3). (See [LapRaZu1, esp., Chs. 3-5].)

3.3.4. Invariance of the complex dimensions under embedding into higherdimensional spaces. Let $(A, \Omega)$ be an RFD in $\mathbb{R}^{N}$ and let $M \geq 1$ be an arbitrary integer. Denote by $(A, \Omega)_{M}$ the natural embedding of $(A, \Omega)$ into $\mathbb{R}^{N+M}$, where $(A, \Omega)_{M}:=\left(A_{M}, \Omega_{M}\right)$, with

$$
A_{M}:=A \times\{0\} \times \cdots \times\{0\} \subseteq \mathbb{R}^{N+M}
$$

and 69

$$
\Omega_{M}:=\Omega \times(-1,1)^{M} \subseteq \mathbb{R}^{N+M} .
$$

Then, it is shown in LapRaZu1, §4.7.2] that given any connected open neighborhood $U$ of the critical line $\{\operatorname{Re}(s)=\bar{D}\}$, where (as usual)

$$
\bar{D}:=\overline{\operatorname{dim}}_{B} A=D\left(\widetilde{\zeta}_{A}\right)=D\left(\zeta_{A}\right),
$$

with $\bar{D}<N$, the tube zeta function $\widetilde{\zeta}_{A, \Omega}$ has a (necessarily unique) meromorphic extension to $U$ if and only if $\widetilde{\zeta}_{(A, \Omega)_{M}}$ does, and in that case, the visible complex dimensions of the $\operatorname{RFD}(A, \Omega)$ in $\mathbb{R}^{N}$ and of the $\operatorname{RFD}(A, \Omega)_{M}$ in $\mathbb{R}^{N+M}$ coincide (and similarly for the distance zeta functions $\zeta_{A, \Omega}$ and $\zeta_{(A, \Omega)_{M}}$ ):

$$
\begin{aligned}
\mathcal{D}_{A, \Omega}(U) & =\mathcal{D}\left(\widetilde{\zeta}_{A, \Omega} ; U\right)=\mathcal{D}\left(\widetilde{\zeta}_{(A, \Omega)_{M}} ; U\right) \\
& =\mathcal{D}\left(\zeta_{A, \Omega} ; U\right)=\mathcal{D}\left(\zeta_{(A, \Omega)_{M}} ; U\right)=\mathcal{D}_{(A, \Omega)_{M}}(U),
\end{aligned}
$$

as equalities between multisets. Moreover,

$$
\begin{aligned}
\mathcal{D}_{P C}(A, \Omega) & =\operatorname{dim}_{P C}(A, \Omega)=\mathcal{D}_{P C}\left(\widetilde{\zeta}_{A, \Omega}\right)=\mathcal{D}_{P C}\left(\widetilde{\zeta}_{(A, \Omega)_{M}}\right) \\
& =\mathcal{D}_{P C}\left(\zeta_{A, \Omega}\right)=\mathcal{D}_{P C}\left(\zeta_{(A, \Omega)_{M}}\right)=\operatorname{dim}_{P C}\left(\zeta_{(A, \Omega)_{M}}\right)=\mathcal{D}_{P C}(A, \Omega)_{M}
\end{aligned}
$$

and

$$
\begin{aligned}
\bar{D}: & =\overline{\operatorname{dim}}_{B}(A, \Omega)=D\left(\widetilde{\zeta}_{A, \Omega}\right)=D\left(\widetilde{\zeta}_{(A, \Omega)_{M}}\right) \\
& =D\left(\zeta_{A, \Omega}\right)=D\left(\zeta_{(A, \Omega)_{M}}\right)=\overline{\operatorname{dim}}_{B}(A, \Omega)_{M} .
\end{aligned}
$$
of $\mathbb{R}^{{ }^{M}}$.

${ }^{69}$ Here, $\{0\} \times \cdots \times\{0\}$ is the $M$-fold Cartesian product of $\{0\}$ by itself, viewed as a subset 
Consequently, neither the values nor the multiplicities of the (visible) complex dimensions (and, in particular, of the principal complex dimensions) of the RFD $(A, \Omega)$ depend on the dimension of the ambient space 70

Moreover, we point out that since $\overline{\operatorname{dim}}_{B}(A, \Omega)<N$ (and hence also, $\overline{\operatorname{dim}}_{B}(A, \Omega)_{M}$ $<N+M)$, the exact same results hold for the tube zeta functions $\widetilde{\zeta}_{A, \Omega}$ and $\widetilde{\zeta}_{(A, \Omega)_{M}}$ replaced, respectively, by the distance zeta functions $\zeta_{A, \Omega}$ and $\zeta_{(A, \Omega)_{M}}$.

Finally, we note that as usual, the exact analog of the results stated in this subsection (i.e., $\sqrt{3.3 .4}$ ) hold for the special case of bounded subsets $A$ (instead of $\operatorname{RFDs}(A, \Omega))$ in $\mathbb{R}^{N}$. It suffices to replace the RFDs $(A, \Omega)$ and $(A, \Omega)_{M}$ by the bounded sets $A$ and $A_{M}$ (as given by (3.59)) in $\mathbb{R}^{N}$ and $\mathbb{R}^{N+M}$, respectively, in all of the corresponding statements; see [LapRaZu1, §4.7.1] for the details.

3.4. Examples of fractal zeta functions and complex dimensions. In this subsection, we give a variety of examples of bounded sets and of RFDs for which the associated distance zeta function (or, equivalently, in light of the functional equation (3.11) or (3.15), the tube zeta function) can be calculated explicitly and the corresponding poles (i.e., the complex dimensions) can be determined. We will limit ourselves to the simplest examples and omit the computation involved, often based in part on symmetry and scaling considerations, but refer instead to LapRaZu1 or to LapRaZu2-9 for the details and many further examples.

3.4.1. The Sierpinski gasket. Let $A \subseteq \mathbb{R}^{2}$ be the classic Sierpinski gasket. It is a self-similar set in $\mathbb{R}^{2}$ with three equal scaling ratios $r_{1}=r_{2}=r_{3}=1 / 2$ and hence, with Minkowski dimension $D=\operatorname{dim}_{B} A=\log 3 / \log 2=\log _{2} 3$ (coinciding with the similarity dimension of $A$ ). As is well-known, $A$ is the unique (nonempty) compact subset of $\mathbb{R}^{2}$ satisfying the fixed point equation

$$
A=\bigcup_{j=1}^{3} S_{j}(A),
$$

where $S_{1}, S_{2}, S_{3}$ are contractive similarity transformations of $\mathbb{R}^{2}$ (with scaling ratios $r_{1}, r_{2}, r_{3}$, as above) defined in a simple way and with respective fixed points $v_{1}, v_{2}, v_{3}$, the vertices of the unit equilateral triangle, which is the generator of $A$.

Then, one can show (see LapRaZu1, §5.5.3]) that for $\delta>1 / 4 \sqrt{3}$ (so that $A_{\delta}$ be connected) ${ }^{71} \zeta_{A}$ has a meromorphic extension to all of $\mathbb{C}$ given by

$$
\zeta_{A}(s)=\frac{6(\sqrt{3})^{1-s} 2^{-s}}{s(s-1)\left(2^{s}-3\right)}+2 \pi \frac{\delta^{s}}{s}+3 \frac{\delta^{s-1}}{s-1}
$$

${ }^{70}$ It is significantly simpler to check that the values of $\operatorname{dim}_{B}(A, \Omega)$, and also the statements according to which $(A, \Omega)$ is Minkowski nondegenerate or is Minkowski measurable, are independent of the dimension of the ambient space; see LapRaZu1 \$4.7.2]. In the Minkowski measurable case, the corresponding Minkowski content can be suitably normalized (much as in Fed2 ) so as to also be independent of the embedding dimension; see Res. (And similarly for the normalized values of $\mathcal{M}_{*}$ and $\mathcal{M}^{*}$.)

${ }^{71}$ Recall from $\$ 3.2$ that the poles of $\zeta_{A, \Omega}$ (and of $\widetilde{\zeta}_{A, \Omega}$ ) are independent of the choice of $\delta>0$; i.e., the set of complex dimensions of an $\operatorname{RFD}(A, \Omega), \mathcal{D}_{A, \Omega}=\mathcal{D}\left(\zeta_{A, \Omega}\right)=\mathcal{D}\left(\widetilde{\zeta}_{A, \Omega}\right)$, is independent of the choice of $\delta>0$. It is also true, in particular, for a bounded set $A$ instead of an $\operatorname{RFD}(A, \Omega)$ (by considering the RFD $\left(A, A_{\delta_{2}}\right)$, for any fixed $\left.\delta_{2}>0\right)$. This comment, being valid for any RFD $(A, \Omega)$ (and, in particular, bounded set) in $\mathbb{R}^{N}$, will no longer be repeated in this section (i.e., 3.4. 
for every $s \in \mathbb{C}$. Consequently, the set of principal complex dimensions of the Sierpinski gasket $A$ is given by

$$
\begin{aligned}
\operatorname{dim}_{P C} A=\mathcal{D}_{P C}\left(\zeta_{A}\right) & =\left\{\log _{2} 3+i \frac{2 \pi}{\log 2} k: k \in \mathbb{Z}\right\} \\
& =\log _{2} 3+i \frac{2 \pi}{\log 2} \mathbb{Z}
\end{aligned}
$$

and the set of all complex dimensions of $A$ is given by

$$
\begin{aligned}
\mathcal{D}_{A}:=\mathcal{D}\left(\zeta_{A}\right) & =\{0\} \cup\left(\log _{2} 3+i \frac{2 \pi}{\log 2} \mathbb{Z}\right)=\{0\} \cup \operatorname{dim}_{P C} A \\
& =\{0\} \cup\left\{s_{k}:=\log _{2} 3+i \frac{2 \pi}{\log 2} k: k \in \mathbb{Z}\right\} .
\end{aligned}
$$

Each complex dimension 0 or $s_{k}:=\log _{2} 3+i(2 \pi / \log 2) k(k \in \mathbb{Z})$ is simple (i.e., is a simple pole of $\zeta_{A}$ ) and the corresponding residue is given respectively by 72

$$
\operatorname{res}\left(\zeta_{A}, 0\right)=3 \sqrt{3}+2 \pi, \operatorname{res}\left(\zeta_{A}, 1\right)=0
$$

and for each $k \in \mathbb{Z}$,

$$
\operatorname{res}\left(\zeta_{A}, s_{k}\right)=\frac{6(\sqrt{3})^{1-s_{k}}}{(\log 2) 4^{s_{k}} s_{k}\left(s_{k}-1\right)} .
$$

Finally, note that in (3.67) and (3.68), $D:=\log _{2} 3$ is the Minkowski dimension of $A$ and $\mathbf{p}:=2 \pi / \log 2$ is the oscillatory period of $A$. Also, the expression obtained for $\operatorname{dim}_{P C} A$ in (3.67) is compatible with the results of part (c) of 3.3 .2 see (3.45).

3.4.2. The Sierpinski carpet. Let $A \subseteq \mathbb{R}^{2}$ be the classic Sierpinski carpet (with generator the unit square and 8 equal scaling ratios $\left.r_{1}=\cdots=r_{8}=1 / 3\right)$. As is well known, $A$ is the unique (nonempty) compact subset of $\mathbb{R}^{2}$ such that $A=\cup_{j=1}^{8} S_{j}(A)$, where $S_{1}, \cdots, S_{8}$ are suitable contractive similarities of $\mathbb{R}^{2}$. Clearly, $D:=\operatorname{dim}_{B} A$ exists and $D=\log _{3} 8$, the similarity dimension of the self-similar set $A$. Then, much as in the case of the Sierpinski gasket $A$ from 3.4 .1 just above, it can be shown (see LapRaZu1, Prop. 3.21]) that $\zeta_{A}$ has a meromorphic extension to all of $\mathbb{C}$, and that for every $\delta>1 / 6$ (so that the $\delta$-neighborhood $A_{\delta}$ of $A$ be connected) and every $s \in \mathbb{C}$,

$$
\zeta_{A}(s)=\frac{8}{2^{s} s(s-1)\left(3^{s}-8\right)}+2 \pi \frac{\delta^{s}}{s}+4 \frac{\delta^{s-1}}{s-1} .
$$

It follows that

$$
\operatorname{dim}_{P C} A=\mathcal{D}_{P C}\left(\zeta_{A}\right)=\left\{\log _{3} 8+i \frac{2 \pi}{\log 3} k: k \in \mathbb{Z}\right\}=\log _{3} 8+i \frac{2 \pi}{\log 3} \mathbb{Z}
$$

\footnotetext{
${ }^{72} \mathrm{~A}$ priori, in light of (3.66), $s=1$ should be a pole of $\zeta_{A}$. However, a direct computation shows that $\operatorname{res}\left(\zeta_{A}, 1\right)=-3+3=0$, as indicated in (3.69). Hence, 1 is not a complex dimension of the Sierpinski gasket. In fact, the corresponding fractal tube formula will not contain a term corresponding to $s=1$; see the relevant parts of 3.5.3 along with LapRaZu1 \$5.5.3], especially, the last equation before Example 5.5.13 in loc. cit.
} 
and the set of all complex dimensions of the Sierpinski carpet is given by

$$
\begin{aligned}
\mathcal{D}_{A}=\mathcal{D}\left(\zeta_{A}\right) & =\{0,1\} \cup\left(\log _{3} 8+i \frac{2 \pi}{\log 3} \mathbb{Z}\right) \\
& =\{0,1\} \cup \operatorname{dim}_{P C} A=\{0,1\} \cup\left\{s_{k}:=\log _{3} 8+i \frac{2 \pi}{\log 3} k: k \in \mathbb{Z}\right\} .
\end{aligned}
$$

Furthermore, the complex dimensions of $A$ are all simple and the residues at 0,1 and $s_{k}(k \in \mathbb{Z})$ are given, respectively, by

$$
\operatorname{res}\left(\zeta_{A}, 0\right)=2 \pi+\frac{8}{7}, \operatorname{res}\left(\zeta_{A}, 1\right)=\frac{16}{5}
$$

and

$$
\operatorname{res}\left(\zeta_{A}, s_{k}\right)=\frac{2^{-s_{k}}}{(\log 3) s_{k}\left(s_{k}-1\right)}, \text { for all } k \in \mathbb{Z} .
$$

Again, in (3.72) and (3.73), $D:=\log _{3} 8$ and $\mathbf{p}:=2 \pi / \log 3$ are, respectively, the Minkowski dimension and the oscillatory period of $A$, in agreement with the results stated in part $(c)$ of 3.3 .2

3.4.3. The 3-d Sierpinski carpet. We refer to [LapRaZu1, Exple. 5.5.13] for the precise definition of this version of the three-dimensional Sierpinski carpet $A$ and for the corresponding results. It is shown there that for any $\delta>1 / 6$ (so that $A_{\delta}$ be connected), $\zeta_{A}$ has a meromorphic extension to all of $\mathbb{C}$ given by

$$
\zeta_{A}(s)=\frac{48 \cdot 2^{-s}}{s(s-1)(s-2)\left(3^{s}-26\right)}+4 \pi \frac{\delta^{s}}{s}+6 \pi \frac{\delta^{s-1}}{s-1}+6 \frac{\delta^{s-2}}{s-2},
$$

for every $s \in \mathbb{C}$. Therefore,

$$
\operatorname{dim}_{P C}\left(\zeta_{A}\right)=\mathcal{D}_{P C}\left(\zeta_{A}\right)=\log _{3} 26+i \frac{2 \pi}{\log 3} \mathbb{Z}
$$

and

$$
\mathcal{D}\left(\zeta_{A}\right)=\{0,1,2\} \cup \operatorname{dim}_{P C} A=\{0,1,2\} \cup\left\{s_{k}:=D+i k \mathbf{p}: k \in \mathbb{Z}\right\},
$$

where $D:=D\left(\zeta_{A}\right)=\log _{3} 26$ and $\mathbf{p}:=2 \pi / \log 3$ are, respectively, the Minkowski dimension (as well as the similarity dimension) and the oscillatory period of $A$. Each complex dimension in (3.77) and (3.78) is simple and

$$
\operatorname{res}\left(\zeta_{A}, j\right)=4 \pi-\frac{24}{25}, 6 \pi+\frac{24}{23}, \frac{96}{17} \text { for } j=0,1,2,
$$

respectively; also, for every $k \in \mathbb{Z}$,

$$
\operatorname{res}\left(\zeta_{A}, s_{k}\right)=\frac{24}{13.2^{s_{k}} s_{k}\left(s_{k}-1\right)\left(s_{k}-2\right) \log 3} .
$$


3.4.4. The $N$-dimensional relative Sierpinski gasket. Let $\left(A_{N}, \Omega_{N}\right)$ denote the $N$-dimensional relative Sierpinski gasket, also called the (inhomogeneous) $N$-gasket RFD, in short, and as introduced in LapRaZu1, Exple. 4.2.26] (as well as in LapRaZu4). We refer the interested reader to loc. cit. for a detailed description of its geometric construction and for the corresponding figures. (See also Remark 3.8 below for a synopsis of the construction.) We simply mention here that for each fixed integer $N \geq 2,\left(A_{N}, \Omega_{N}\right)$ is an RFD in $\mathbb{R}^{N}$ which can also be viewed as a self-similar spray (or RFD) in $\mathbb{R}^{N}$ (in the refined sense of [LapRaZu1, §4.2.1 and §5.5.6] rather than in the original sense of Lap3, LapPo3 and [LapPe2 or [LapPeWi1]), with $N+1$ equal scaling ratios $r_{1}=\cdots=r_{N+1}=1 / 2$ and with a single generator RFD $\left(\partial \Omega_{N, 0}, \Omega_{N, 0}\right)$, where the bounded open set $\Omega_{N, 0}$ in $\mathbb{R}^{N}$ (called the $N$-plex) is described in Remark 3.8. Furthermore, unlike in our previous examples in $3.4 .13 .4 .3, A_{N}$ is not a self-similar set (in the usual sense of the term) but is instead an inhomogeneous self-similar set, in the sense of BarnDemk and (with a different terminology) of [Hat. More specifically, it is the unique (nonempty) compact subset $A$ of $\mathbb{R}^{N}$ satisfying the inhomogeneous fixed point equation

$$
A=\bigcup_{j=1}^{N+1} S_{j}(A) \cup B,
$$

where the maps $S_{j}$ (for $j=1, \cdots, N+1$ ) are $N+1$ contractive similarity transformation 73 of $\mathbb{R}^{N}$ (the same ones as those defining the $N$-dimensional analog of the usual self-similar gasket, which is an homogeneous or a classic self-similar set, satisfying the counterpart of (3.81) with $B:=\emptyset$, the empty set) and $B$ is a certain nonempty compact subset of $\mathbb{R}^{N}$; in fact, $B$ can be chosen to be equal to $\partial \Omega_{N, 0}$, the boundary of the $N$-plex $\Omega_{N, 0}$ described in Remark 3.8

REMARK 3.8. (Construction of the generator $\Omega_{N, 0}$ and of the inhomogeneous $N$-gasket RFD $\left(A_{N}, \Omega_{N}\right)$.) Here, the generator $\Omega_{N, 0}$ and the compact set $A_{N}$ can be constructed as follows. Let $V_{N}=\left\{P_{1}, \cdots, P_{N+1}\right\}$ be a set of $N+1$ points in $\mathbb{R}^{N}$ such that $\left|P_{j}-P_{k}\right|=1$, for any $j \neq k$. (Such a set can be constructed inductively.) Let $\Omega_{N}$ be the (necessarily closed) convex hull of $V_{N}$. Clearly, $\Omega_{N}$ is an $N$-simplex. Then, $\Omega_{N, 0}$, called the $N$-plex, is the bounded open subset of $\mathbb{R}^{N}$ obtained by taking the interior of the convex hull of the set of midpoints of all of the $\frac{(N+1) N}{2}=\left(\begin{array}{c}N \\ 2\end{array}\right)$ edges of the $N$-simplex $\Omega_{N}$. (Note that for $N=2, \Omega_{N, 0}$ is the first deleted open triangle in the construction of the Sierpinski gasket, while for $N=3$, it is an octahedron; see LapRaZu1, Fig. 4.7] for an illustration.)

Now, the set $\overline{\Omega_{N}} \backslash \Omega_{N, 0}$ is the union of $N+1$ congruent and compact $N$-simplices with disjoint interiors and having all their sides (edges) of length $1 / 2$. This is the first step in the construction of $A_{N}$. We proceed analogously with each of the aforementioned $N$-simplices. We then repeat the construction, ad infinitum. The compact subset $A_{N}$ of $\mathbb{R}^{N}$ obtained in this manner is called the inhomogeneous $N$-gasket. For $N=2$, it coincides with the classic Sierpinski gasket (studied in (3.4.1), but when $N \geq 3$, it does not coincide with the usual $N$-dimensional Sierpinski gasket (studied, e.g., in KiLap1). In fact, still for $N \geq 3$, it is no longer self-similar (in the classic sense) but is instead an inhomogeneous self-similar set

\footnotetext{
${ }^{73}$ These similitudes $\left(S_{j}\right)_{j=1}^{N+1}$ have for respective fixed points $\left(P_{j}\right)_{j=1}^{N+1}$, the points chosen at the beginning of Remark 3.8
} 
satisfying (3.81), with $B:=\partial \Omega_{N, 0}$, the boundary of the $N$-plex. (See LapRaZu1, Fig. 4.8] for an illustration of the case when $N=3$.)

Finally, the relative (or inhomogeneous) $N$-gasket $R F D$ is given by $\left(A_{N}, \Omega_{N}\right)$, where $A_{N}$ is the above inhomogeneous $N$-gasket and $\Omega_{N}$ is the above $N$-simplex.

Then (see LapRaZu1, Exple. 4.2.26]), for the inhomogeneous $N$-gasket RFD $\left(A_{N}, \Omega_{N}\right)$, the distance zeta function $\zeta_{A_{N}, \Omega_{N}}$ has a meromorphic extension to all of $\mathbb{C}$, given for every $s \in \mathbb{C}$ by

$$
\zeta_{A_{N}, \Omega_{N}}(s)=\frac{g_{N}(s)}{s(s-1) \cdots(s-(N-1))\left(1-(N+1) 2^{-s}\right)},
$$

for some nowhere vanishing entire function $g_{N}$. (For example, when $N=3$, we have $g_{3}(s):=8(\sqrt{3})^{3-s}(2 \sqrt{2})^{-s}$ and if $N=2, g_{2}(s):=6(\sqrt{3})^{1-s} 2^{-s}$, still for all $s \in \mathbb{C}$; see, respectively, LapRaZu1, Eq. (4.2.89) and Prop. 4.2.25].)

In order to explain the form of $\zeta_{A_{N}, \Omega_{N}}$ given in (3.82), we recall that $\left(A_{N}, \Omega_{N}\right)$ is a self-similar RFD with generator the RFD $\left(\partial \Omega_{N, 0}, \Omega_{N, 0}\right)$ and with equal scaling ratios $r_{j} \equiv 1 / 2$, for $j=1, \cdots, N+1$. Thus, according to the results of LapRaZu1, $\S 4.2 .1$ and $\S 5.5 .6]$ about self-similar sprays (and recalled in $\$ 3.4 .10$ below),

$$
\zeta_{A_{N}, \Omega_{N}}(s)=\zeta_{\mathfrak{s}}(s) \cdot \zeta_{\partial \Omega_{N, 0}, \Omega_{N, 0}}(s),
$$

where the scaling zeta function $\zeta_{\mathfrak{s}}$ (here, the geometric zeta function of the underlying unbounded self-similar string with equal scaling ratios $r_{j} \equiv 1 / 2$ for $j=$ $1, \cdots, N+1)$ is given by

$$
\zeta_{\mathfrak{s}}(s)=\frac{1}{1-(N+1) 2^{-s}}
$$

for all $s \in \mathbb{C}$, and where via a direct computation 74 one can show that $\zeta_{\partial \Omega_{N, 0}, \Omega_{N, 0}}$ is given for all $s \in \mathbb{C}$ by

$$
\zeta_{\partial \Omega_{N, 0}, \Omega_{N, 0}}(s)=\frac{g_{N}(s)}{s(s-1) \cdots(s-(N-1))},
$$

with $g_{N}$ as above. Now combining (3.83)-(3.85), we obtain (3.82), as desired.

Next, since $g_{N}$ is nowhere vanishing and is entire, we deduce from (3.82) that

$$
\mathcal{D}_{A, \Omega}:=\mathcal{D}\left(\zeta_{A, \Omega}\right)=\{0,1, \cdots, N-1\} \cup \mathcal{D}\left(\zeta_{\mathfrak{s}}\right),
$$

where (in light of (3.84)

$$
\mathcal{D}\left(\zeta_{\mathfrak{s}}\right)=\log _{2}(N+1)+i \frac{2 \pi}{\log 2} \mathbb{Z} .
$$

Note that, by (3.85),

$$
\mathcal{D}\left(\zeta_{\partial \Omega_{N, 0}, \Omega_{N, 0}}\right)=\{0,1, \cdots, N-1\} .
$$

Therefore, for every $N \geq 2$, the set of complex dimensions of the inhomogeneous $N$-gasket is given by

$$
\mathcal{D}\left(A_{N}, \Omega_{N}\right):=\mathcal{D}\left(\zeta_{A_{N}, \Omega_{N}}\right)=\{0,1, \cdots, N-1\} \cup\left(\log _{2}(N+1)+i \frac{2 \pi}{\log 2} \mathbb{Z}\right) .
$$

\footnotetext{
${ }^{74}$ See loc. cit. for the case when $N=2$ or when $N=3$.
} 
Except when $\log _{2}(N+1)=j$, for some $j \in\{0,1, \cdots, N-1\}$ (i.e., $N=2^{j}-1$, for some $j \in\{2, \cdots, N-1\}$, since $N \geq 2$ here), all of the complex dimensions of the $\operatorname{RFD}\left(A_{N}, \Omega_{N}\right)$ in (3.86) are simple 75

It is instructive (although easy) to determine $\operatorname{dim}_{B}\left(A_{N}, \Omega_{N}\right)$ and $\operatorname{dim}_{P C}\left(A_{N}, \Omega_{N}\right)$. In light of (3.83), we have that

$$
\overline{\operatorname{dim}_{B}}\left(A_{N}, \Omega_{N}\right)=\max \left(D\left(\zeta_{\partial \Omega_{N, 0}, \Omega_{N, 0}}\right), D\left(\zeta_{\mathfrak{s}}\right)\right)=\max \left(N-1, \log _{2}(N+1)\right) .
$$

Observe that in (3.90), $\operatorname{dim}_{B}\left(\partial \Omega_{N, 0}, \Omega_{N, 0}\right)=N-1$ is the Minkowski dimension (which exists) of the generating $\operatorname{RFD}\left(\partial \Omega_{N, 0}, \Omega_{N, 0}\right)$ and $\sigma_{N}:=N-1$ is the similarity dimension of the self-similar spray or $\operatorname{RFD}\left(A_{N}, \Omega_{N}\right)$. Also, since one can show that $\operatorname{dim}_{B}\left(A_{N}, \Omega_{N}\right)$ exists, we conclude that

$$
\begin{aligned}
D:=\operatorname{dim}_{B}\left(A_{N}, \Omega_{N}\right)\left(=D\left(\zeta_{A_{N}, \Omega_{N}}\right)\right) & =\max \left(N-1, \log _{2}(N+1)\right) \\
& = \begin{cases}\log _{2} 3, & \text { for } N=2, \\
N-1, & \text { for } N \geq 3 .\end{cases}
\end{aligned}
$$

Furthermore, we deduce from (3.89) and (3.91) that the set of principal complex dimensions of $\left(A_{N}, \Omega_{N}\right)$ is given by

$$
\operatorname{dim}_{P C}\left(A_{N}, \Omega_{N}\right)= \begin{cases}\log _{2} 3+i \frac{2 \pi}{\log 2} \mathbb{Z}, & \text { for } N=2, \\ 2+i \frac{2 \pi}{\log 2} \mathbb{Z}, & \text { for } N=3, \\ \{N-1\}, & \text { for } N \geq 4 .\end{cases}
$$

Observe that for $N=2$, we have that $\sigma_{2}=\log _{2} 3>\operatorname{dim}_{B}\left(\partial \Omega_{2,0}, \Omega_{2,0}\right)=1$, and hence, $\operatorname{dim}\left(A_{2}, \Omega_{2}\right)=\sigma_{2}$, the similarity dimension of the Sierpinski gasket, in agreement with a well-known result about classic or homogeneous self-similar sets (satisfying the open set condition); see, e.g., Hut or [Fa1, Ch. 9]. By contrast, when $N \geq 4$, we have the reverse inequality ${ }^{76}$ namely,

$$
\sigma_{N}=\log _{2}(N+1) \leq \operatorname{dim}_{B}\left(\partial \Omega_{N, 0}, \Omega_{N, 0}\right)=N-1 .
$$

Therefore, $\operatorname{dim}_{B}\left(A_{N}, \Omega_{N}\right)=\operatorname{dim}_{B}\left(\partial \Omega_{N, 0}, \Omega_{N, 0}\right)=N-1$, in this case 77

Finally, if $N=3$, we have $\sigma_{3}=\operatorname{dim}_{B}\left(\partial \Omega_{3,0}, \Omega_{3,0}\right)=2$. This coincidence between the geometry of the generator $\left(\partial \Omega_{3,0}, \Omega_{3,0}\right)$ and the scaling of the self-similar spray $\left(A_{3}, \Omega_{3}\right)$ explains why $D=2$ is a complex dimension of multiplicity two if $N=3$. In some sense, one can say that there is a resonance between the underlying geometry and the underlying scaling of the relative 3-gasket $\operatorname{RFD}\left(A_{3}, \Omega_{3}\right)$.

The above facts have interesting geometric consequences, as is explained in detail in LapRaZu1, §5.5.6], by using either the fractal tube formulas of LapRaZu1, $\S 5.1-\S 5.3]$ or the Minkowski measurability criteria of [LapRaZu1, §5.4] (both to be briefly discussed in 33.5$)$. Firstly, if $N=2$, the RFD $\left(A_{2}, \Omega_{2}\right)$ is not Minkowski measurable because in light of (3.92), it has nonreal principal complex dimensions; however, $\left(A_{2}, \Omega_{2}\right)$ is Minkowski nondegenerate. Secondly, if $N \geq 4$ (and since then,

\footnotetext{
${ }^{75}$ In view of the aforementioned results of loc. cit., the residues of $\zeta_{A_{N}, \Omega_{N}}$ at each complex dimension $\omega_{j}=j$ (for $j \in\{0,1, \cdots, N-1\}$ and $s_{k}:=\log _{2}(N+1)+i(2 \pi / \log 2) k(k \in \mathbb{Z})$ can be explicitly computed when $N=2$ and when $N=3$; see LapRaZu1, Exple. 4.2.24 and Eq. $(4.2 .88)]$.

${ }^{76}$ In fact, this inequality 3.93 is always strict; indeed, it is easy to check by induction on $N$ that we never have $N=2^{N-1}-1$, for some integer $N \geq 4$.

${ }^{77}$ There is no contradiction because, as we recall from our earlier discussion, $\left(A_{N}, \Omega_{N}\right)$ is an inhomogeneous (but unless $N=2$ ) is not a standard (or homogeneous) self-similar set.
} 
$2^{N-1} \neq N-1$, so that the dimension $D=N-1$ of $\left(A_{N}, \Omega_{N}\right)$ is simple), the RFD is not Minkowski measurable but is still Minkowski nondegenerate.

Lastly, if $N=3,\left(A_{3}, \Omega_{3}\right)$ is not Minkowski measurable (since its Minkowski dimension $D=2$ has multiplicity two); further, it is also Minkowski degenerate, which suggests that the usual power law is no longer appropriate to measure the "fractality" of $\left(A_{3}, \Omega_{3}\right) 78$ However, one can use a suitably generalized Minkowski content (as in HeLap and LapRaZu1, §6.1.1.2]), involving the choice of the gauge function $h(t):=\log \left(t^{-1}\right)$ for all $t \in(0,1)$, so that the RFD $\left(A_{3}, \Omega_{3}\right)$ be not only Minkowski nondegenerate but also Minkowski measurable, relative to $h$ (by contrast with the cases when $N=2$ and $N=3$ ); see [LapRaZu1, Thm. 5.4.27].

EXERCISE 3.9. Verify that when $N=3$, we have

$$
g_{3}(s)=8(\sqrt{3})^{3-s}(2 \sqrt{2})^{-s},
$$

for all $s \in \mathbb{C}$ in (3.82) and (3.85).

EXERCISE 3.10. Calculate the fractal zeta functions and the complex dimensions of the $N$-carpet $\operatorname{RFD}(A, \Omega)$ (the $N$-dimensional relative Sierpinski carpet), which extends to $\mathbb{R}^{N}$ both the Sierpinski carpet $(N=2$; see 33.4.2) and 3-carpet $(N=3$; see $\oint 3.4 .3)$.

Note that this example is significantly simpler than that of the $N$-gasket RFD studied in the present subsection (i.e., 33.4.4); indeed, unlike for the relative $N$ gasket, which is an inhomogeneous self-similar set, the compact set $A$ is an homogeneous (i.e., classical) self-similar set in $\mathbb{R}^{N}$. In fact, $A$ coincides with the standard $N$-Sierpinski carpet, while $\Omega=(0,1)^{N}$.

We refer the interested reader to LapRaZu1 Exple. 4.2.31] for the complete answers and the corresponding computation. We simply mention here that

$$
\operatorname{dim}_{P C}(A, \Omega)=\log _{3}\left(3^{N}-1\right)+i \frac{2 \pi}{\log 3} \mathbb{Z},
$$

where, as before, $D:=\log _{3}\left(3^{N}-1\right)$ is the Minkowski dimension of $(A, \Omega)$ and $\mathbf{p}:=2 \pi / \log 3$ is the oscillatory period of $(A, \Omega)$, while

$$
\mathcal{D}_{A, \Omega}=\mathcal{D}\left(\zeta_{A, \Omega}\right)=\{0,1, \cdots, N-1\} \cup \operatorname{dim}_{P C}(A, \Omega) .
$$

Furthermore, in either (3.95) or (3.96), each complex dimension is simple.

3.4.5. The $\frac{1}{2}$-square and $\frac{1}{3}$-square fractals. We discuss here in parallel two related relative fractal drums, namely, the $\frac{1}{2}$-square and $\frac{1}{3}$-square fractals, which exhibit somewhat different properties.

(a) (The $\frac{1}{2}$-square fractal). Starting with the closed unit square $[0,1]^{2} \subseteq \mathbb{R}^{2}$, we remove the two open squares of side length $\frac{1}{2}$, denoted by $G_{1}$ and $G_{2}$, along the main diagonal. Next, we repeat this step with the two remaining closed squares of side length $1 / 2$; and so on, ad infinitum. The $\frac{1}{2}$-square fractal $A$ is the compact set that is left at the end of the process. (See also [LapRaZu1, Fig. 4.10] for an illustration.)

The $\frac{1}{2}$-square fractal is a nonhomogeneous self-similar fractal (as was the case of the set $A$ in the construction of the relative Sierpinski $N$-gasket in $\$ 3.4 .4$; more

\footnotetext{
${ }^{78}$ All of these facts are established in LapRaZu1 \$5.5.6]; see, especially, part (c) of Remark 5.5.26 of loc. cit..
} 
specifically, it is the unique nonempty compact subset $A$ of $\mathbb{R}^{2}$ satisfying the following inhomogeneous fixed point equation:

$$
A=\bigcup_{j=1}^{2} S_{j}(A) \cup B,
$$

where the nonempty compact set $B \subseteq \mathbb{R}^{2}$ is the union of the left and upper sides of the closed square $\bar{G}_{1}$ and of the right and lower sides of the closed square $\bar{G}_{2}$. Here, the contractive similitudes of $\mathbb{R}^{2}$ involved, namely, the maps $S_{1}$ and $S_{2}$, have respective fixed points at the lower left vertex and the upper right vertex of the unit square, and have scaling ratios $r_{1}=r_{2}=1 / 279$ (See [LapRaZu1, Fig. 4.11] for an illustration.)

Let $\Omega:=(0,1)^{2}$ and consider the $\operatorname{RFD}(A, \Omega)$; by construction, it is a self-similar spray (or RFD) with generator $G=G_{1} \cup G_{2}$ and scaling ratios $r_{1}=r_{2}=1 / 2$.

It is shown in LapRaZu1, Exple. 4.2.33] that for every $\delta>1 / 4, \zeta_{A, \Omega}$ and hence also, $\zeta_{A}$, in light of (3.99) below, have a meromorphic continuation to all of $\mathbb{C}$ given for every $s \in \mathbb{C}$ respectively by

$$
\zeta_{A, \Omega}(s)=\frac{\zeta_{\partial G, G}(s)}{1-2 \cdot 2^{-s}}=\frac{2^{-(s+1)}}{s(s-1)\left(2^{s-1}-1\right)}
$$

and

$$
\begin{aligned}
\zeta_{A}(s) & =\zeta_{A, \Omega}(s)+\zeta_{[0,1]^{2}}(s) \\
& =\frac{2^{-(s+1)}}{s(s-1)\left(2^{s-1}-1\right)}+4 \frac{\delta^{s-1}}{s-1}+2 \pi \frac{\delta^{s}}{s} .
\end{aligned}
$$

It follows that

$$
D:=D\left(\zeta_{A}\right)=\operatorname{dim}_{B} A=D\left(\zeta_{A, \Omega}\right)=\operatorname{dim}_{B}(A, \Omega)=1
$$

and

as well as

$$
\operatorname{dim}_{P C} A=\operatorname{dim}_{P C}(A, \Omega)=1+\frac{2 \pi}{\log 2} \mathbb{Z}
$$

$$
\mathcal{D}_{A}=\mathcal{D}\left(\zeta_{A}\right)=\mathcal{D}_{A, \Omega}=\mathcal{D}\left(\zeta_{A, \Omega}\right)=\{0,1\} \cup\left(1+i \frac{2 \pi}{\log 2} \mathbb{Z}\right),
$$

where these are equalities between multisets.

All of the complex dimensions in (3.101) and (3.102) (namely, 0 and $s_{k}:=$ $1+i(2 \pi / \log 2) k$, for $k \in \mathbb{Z})$ are simple, except for the dimension $D=1$ which is double.

Furthermore, for all $k \in \mathbb{Z} \backslash\{0\}$, we have

$$
\operatorname{res}\left(\zeta_{A}, 0\right)=1+2 \pi \text { and } \operatorname{res}\left(\zeta_{A}, s_{k}\right)=\frac{4^{-i \mathbf{p} k}}{4 s_{k}\left(s_{k}-1\right)},
$$

where $\mathbf{p}:=2 \pi / \log 2$ is the oscillatory period of the self-similar spray $(A, \Omega)$.

(b) (The $\frac{1}{3}$-square fractal). As in part $(a)$, we begin with the unit square $[0,1]^{2}$; we then divide it into nine congruent smaller squares. We further delete seven of

\footnotetext{
${ }^{79}$ It is noteworthy that the homogeneous self-similar set $E$ which is the unique nonempty compact subset of $\mathbb{R}^{2}$ satisfying the homogeneous fixed point equation associated with (3.97) (namely, $E=\cup_{j=1}^{2} S_{j}(E)$ ), is the main diagonal of the unit square $[0,1]^{2}$.
} 
those smaller squares; that is, we only keep the lower and upper right squares. We then repeat the process, ad infinitum. What is left at the end of the process is denoted by $A$ and called the $\frac{1}{3}$-square fractal.

If $\Omega:=(0,1)^{2}$, then we consider the $\operatorname{RFD}(A, \Omega)$ in $\mathbb{R}^{2}$. Note that (as in part $(a)), A$ is an inhomogeneous self-similar fractal; more specifically, it is the unique (nonempty) compact subset of $\mathbb{R}^{2}$ satisfying the following inhomogeneous fixed point equation:

$$
A=\bigcup_{j=1}^{2} S_{j}(A) \cup B,
$$

where $B \subseteq \mathbb{R}^{2}$ is the nonempty compact set defined by $B:=\partial G$ and $G$ (called the generator of the self-similar spray $(A, \Omega)$ ) is a suitable open convex polygon. Furthermore, $S_{1}$ and $S_{2}$ are contractive similitudes of $\mathbb{R}^{2}$, with respective fixed points located at the lower left vertex and the upper right vertex of the unit square.

The $\operatorname{RFD}(A, \Omega)$ is a self-similar spray (or RFD) with generator $G$ and scaling ratios $r_{1}=r_{2}=r_{3}=1 / 3$.

Much as in part (a), it is shown in [LapRaZu1, Exple. 4.2.34] that $\zeta_{A, \Omega}$ (and thus also $\zeta_{A}$ ) admits a meromorphic continuation to all of $\mathbb{C}$ given for every $s \in \mathbb{C}$ (and for all sufficiently large positive $\delta$ ) by

$$
\zeta_{A, \Omega}(s)=\frac{\zeta_{\partial G, G}(s)}{1-2 \cdot 3^{-s}}=\frac{2}{s\left(3^{s}-2\right)}\left(\frac{6}{s-1}+\Psi(s)\right),
$$

where $\Psi$ is a suitable entire function (which is explicitly known), and

$$
\begin{aligned}
\zeta_{A}(s) & =\zeta_{A, \Omega}(s)+\zeta_{[0,1]^{2}}(s) \\
& =\frac{2}{s\left(3^{s}-2\right)}\left(\frac{6}{s-1}+\Psi(s)\right)+4 \frac{\delta^{s-1}}{s-1}+2 \pi \frac{\delta^{s}}{s} .
\end{aligned}
$$

As a result,

$$
D=D_{A}=D\left(\zeta_{A}\right)=D_{A, \Omega}=D\left(\zeta_{A, \Omega}\right)=1
$$

and (see (3.112) below for a more precise statement)

$$
\operatorname{dim}_{P C} A=\operatorname{dim}_{P C}(A, \Omega) \subseteq\{1\} \cup\left(\log _{3} 2+i \frac{2 \pi}{\log 3} \mathbb{Z}\right),
$$

as well as

$$
\{0,1\} \cup F \subseteq \mathcal{D}_{A}=\mathcal{D}\left(\zeta_{A}\right)=\mathcal{D}_{A, \Omega}=\mathcal{D}\left(\zeta_{A, \Omega}\right) \subseteq\{0,1\} \cup\left(\log _{3} 2+i \frac{2 \pi}{\log 3} \mathbb{Z}\right),
$$

each complex dimension in (3.108) and (3.109) being simple. Here, $F$ is a subset of $\log _{3} 2+i(2 \pi / \log 3) \mathbb{Z}$ containing $\log _{3} 2$ and at least finitely many (but more than two) nonreal principal complex dimensions 80 We conjecture that the set $F$ is in fact (countably) infinite and furthermore, that the inclusions in (3.109) should actually be equalities and $\operatorname{dim}_{P C} A=\operatorname{dim}_{P C}(A, \Omega)=\{1\}$.

It is noteworthy that $\log _{3} 2$ is the dimension of the homogeneous self-similar set $E$ associated with (3.104) (i.e., $E=\cup_{j=1}^{2} S_{j}(E)$, with $E \subseteq \mathbb{R}^{2}$ nonempty and compact); indeed, $E$ is just the ternary Cantor set located along the main diagonal of the unit square $[0,1]^{2}$.

${ }^{80}$ At this stage, the inclusion appearing on the left of 3.109 is only verified numerically. The difficulty here is due to the presence of the entire function $\Psi$. 
Finally, a simple computation yields that

$$
\operatorname{res}\left(\zeta_{A}, 0\right)=12+\pi, \operatorname{res}\left(\zeta_{A, 1}\right)=16
$$

and (with $\mathbf{p}:=2 \pi / \log 3$ and $s_{k}:=\log _{3} 2+i(2 \pi / \log 3) k$, for each $\left.k \in \mathbb{Z}\right)$

$$
\operatorname{res}\left(\zeta_{A}, s_{k}\right)=\frac{3^{-i \mathbf{p} k}}{(\log 3) s_{k}}\left(\frac{6}{s_{k}-1}+\Psi\left(s_{k}\right)\right) \text {. }
$$

Therefore, in light of (3.110), we can now specify the statement made in (3.108) by affirming that

$$
\operatorname{dim}_{P C} A=\operatorname{dim}_{P C}(A, \Omega)=\{1\} .
$$

3.4.6. The $(N-1)$-sphere and its associated RFD. In this subsection, we study the complex dimensions of the $(N-1)$-sphere

$$
A:=S^{N-1}=\left\{x \in \mathbb{R}^{N}:|x|=1\right\},
$$

where $|\cdot|$ denotes the Euclidean norm in $\mathbb{R}^{N}$, and of the associated $\operatorname{RFD}(A, \Omega)$, relative to the open unit ball $\Omega$ of $\mathbb{R}^{N}$, called the $(N-1)$-sphere $R F D$. We shall see that the answer obtained in the latter case is very natural. The difference between the answers in the former case (the $(N-1)$-sphere) and the latter case (the $(N-1)$-sphere RFD) is simply due to the fact that in the former case, we consider two-sided $\varepsilon$-neighborhoods of $A=S^{N-1}$ whereas in the latter case, we deal with one-sided (or "inner") $\varepsilon$-neighborhoods of $A=S^{N-1}$.

(a) (The $(N-1)$-sphere). Let $A$ be the $(N-1)$-dimensional sphere, as given by (3.113). Then, in LapRaZu1, Exple. 2.2.21], the tube zeta function $\zeta_{A}$ of $A$ is shown to have a meromorphic extension to all of $\mathbb{C}$ given for every $s \in \mathbb{C}$ and for any fixed $\delta \in(0,1)$ by

$$
\widetilde{\zeta}_{A}(s)=\Theta_{N} \sum_{k=0}^{N}\left(1-(-1)^{k}\right)\left(\begin{array}{l}
N \\
k
\end{array}\right) \frac{\delta^{s-N+k}}{s-(N-k)},
$$

where $\Theta_{N}$ denotes the volume of the unit ball in $\mathbb{R}^{N}$ and the numbers $\left(\begin{array}{c}N \\ k\end{array}\right)$ are the usual binomial coefficients 81 Therefore, independently of the value of $\delta>0$,

$$
\begin{gathered}
D:=D\left(\widetilde{\zeta}_{A}\right)=D\left(\zeta_{A}\right)=\operatorname{dim}_{B} A=N-1, \\
\operatorname{dim}_{P C} A=\{N-1\}
\end{gathered}
$$

and

$$
\mathcal{D}_{A}=\mathcal{D}\left(\widetilde{\zeta}_{A}\right)=\mathcal{D}\left(\zeta_{A}\right)=\left\{N-1, N-3, \cdots, N-\left(2\left[\frac{N-1}{2}\right]+1\right)\right\},
$$

each complex dimension in (3.116) and (3.117) being simple. Note that for $N \geq 1$ odd (respectively, even), the last number in this set is equal to 0 (respectively, 1 ).

Finally, for every $d \in \mathcal{D}_{A}$,

$$
\operatorname{res}\left(\widetilde{\zeta}_{A}, d\right)=2 \Theta_{N}\left(\begin{array}{l}
N \\
d
\end{array}\right) .
$$

\footnotetext{
${ }^{81}$ In light of 3.15), we deduce at once the value of the distance zeta function $\zeta_{A}(s)$ for $s \in \mathbb{C}$.
} 
In particular, for $d:=D=N-1$, a direct computation (based on the definition of the Minkowski content given in $\$ 3.2$ above) yields

$$
\mathcal{M}=\mathcal{M}(A)=2 N \Theta_{N}=\operatorname{res}\left(\widetilde{\zeta}_{A}, D\right)
$$

in agreement with a result stated in part $(a)$ of $\$ 3 . 3 . 2 \longdiv { 8 2 }$ Note that, clearly, $A$ is Minkowski measurable with Minkowski content $\mathcal{M}$.

EXERCiSe 3.11. Show directly that $A=S^{N-1}$ is Minkowski measurable, with Minkowski content $\mathcal{M}$ given by the second equality of (3.119).

(b) (The $(N-1)$-sphere RFD). Consider the $(N-1)$-sphere $\operatorname{RFD}(A, \Omega)$, where $A:=S^{N-1}$ is the unit sphere of $\mathbb{R}^{N}$ (as in part (a) just above) and $\Omega$ is the open unit ball in $\mathbb{R}^{N}$; so that $A=\partial \Omega$ and hence, $(A, \Omega)=(\partial \Omega, \Omega)$. Clearly, for any $N \geq 1$, the $(N-1)$-sphere RFD (or relative $(N-1)$-sphere) is an RFD in $\mathbb{R}^{N}$.

It is shown in [LapRaZu1, Exple. 4.1.19] that the distance zeta function $\zeta_{A, \Omega}$ of $(A, \Omega)$ admits a (necessarily unique) meromorphic extension to all of $\mathbb{C}$ given for every $s \in \mathbb{C}$ and for any fixed $\delta \in(0,1)$ by the following expression 83

$$
\zeta_{A, \Omega}(s)=N \Theta_{N} \sum_{j=0}^{N-1}\left(\begin{array}{c}
N-1 \\
j
\end{array}\right) \frac{(-1)^{N-j-1}}{s-j} .
$$

Therefore, one deduces at once that

$$
\begin{aligned}
D=D\left(\zeta_{A, \Omega}\right)= & D\left(\widetilde{\zeta}_{A, \Omega}\right)=\operatorname{dim}_{B}(A, \Omega)=N-1, \\
& \operatorname{dim}_{P C}=\{N-1\}
\end{aligned}
$$

and

$$
\mathcal{D}_{A, \Omega}=\mathcal{D}\left(\zeta_{A, \Omega}\right)=\mathcal{D}\left(\widetilde{\zeta}_{A, \Omega}\right)=\{0,1, \cdots N-1\} .
$$

Observe the contrast between the result obtained for $\mathcal{D}_{A}$ and $\mathcal{D}_{A, \Omega}$ in (3.117) and (3.123), respectively, as was alluded to in the introduction to this subsection (i.e., 3.4.6). In particular, the set of complex dimensions $\mathcal{D}_{A, \Omega}=\{0,1, \cdots, N-1\}$ of the relative $(N-1)$-sphere obtained in (3.123) is exactly the one we would have expected, a priori.

Finally, for every $j \in\{0,1, \cdots, N-1\}$,

$$
\operatorname{res}\left(\zeta_{A, \Omega}, j\right)=(-1)^{N-j-1} N \Theta_{N}\left(\begin{array}{c}
N-1 \\
j
\end{array}\right) .
$$

In particular, for $j:=D=N-1$, we see that the $\operatorname{RFD}(A, \Omega)$ is Minkowski measurable with (relative) Minkowski content given by

$$
\mathcal{M}=\mathcal{M}(A, \Omega)=(N-D) \operatorname{res}\left(\zeta_{A, \Omega}, D\right)=N \Theta_{N}
$$

note that here, $N-D=1$. (Compare with (3.29).)

\footnotetext{
${ }^{82}$ If the (absolute) $(N-1)$-sphere $A$ had radius $R$ instead of radius 1 , then for any $\delta \in(0, R]$, one should simply substitute $\Theta_{N} R^{d}$ and $\Theta_{N} R^{N-1}$ for $\Theta_{N}$ in 3.118) and (3.119), respectively. (See also part (iii) of Exercise 3.12)

${ }^{83}$ Unlike in part $(a)$ of the present subsection, it is easier to compute directly $\zeta_{A, \Omega}$ rather than $\widetilde{\zeta}_{A, \Omega}$. Of course, in light of (3.11), one can then deduce $\widetilde{\zeta}_{A, \Omega}$ from 3.120 .
} 
EXERCiSE 3.12. (i) Show via a direct computation that $\zeta_{A, \Omega}$ is given by (3.120).

(ii) Address the same question as in $(i)$ for $\widetilde{\zeta}_{A}$ (in part (a) of this subsection) in order to recover the expression stated in (3.114).

(iii) How are the expressions of $\widetilde{\zeta}_{A}$ in (3.114) and of $\zeta_{A, \Omega}$ in (3.120) modified if $A$ and $\Omega$ are, respectively, the $(N-1)$-sphere and the (open) $N$-ball of radius $R$ (instead of radius 1$)$ ?

3.4.7. The Cantor grill. Let $A:=C \times[0,1]$ be the Cartesian product of the ternary Cantor set by the unit interval. Henceforth, $A \subseteq \mathbb{R}^{2}$ is referred to as the Cantor grill. (See LapRaZu1, Fig. 2.15] for an illustration.) Then, according to LapRaZu1, Exple. 2.2.34 in $\S 2.2 .3$,

$$
D=D_{C}=\operatorname{dim}_{B} A=1+\log _{3} 2,
$$

the set of principal complex dimensions of $A$ is given by

$$
\operatorname{dim}_{P C} A=\left(1+\log _{3} 2\right)+i \frac{2 \pi}{\log 3} \mathbb{Z},
$$

while the set of all complex dimensions (in $\mathbb{C}$ ) of $A$ is given by

$$
\begin{aligned}
\mathcal{D}_{A} & =\mathcal{D}\left(\zeta_{A}\right)=\{0,1\} \cup \bigcup_{m=0}^{1}\left(\left(m+\log _{3} 2\right)+i \frac{2 \pi}{\log 3} \mathbb{Z}\right) \\
& =\{0,1\} \cup\left(D_{C}+i \mathbf{p} \mathbb{Z}\right) \cup\left(\left(1+D_{C}\right)+i \mathbf{p} \mathbb{Z}\right),
\end{aligned}
$$

where $D_{C}=\log _{3} 2=\operatorname{dim}_{B} C$ is the Minkowski dimension of the Cantor set $C$ and $\mathbf{p}:=2 \pi / \log 3$ is the oscillatory period of $C$. Each complex dimension in (3.127) and (3.128) is simple.

EXERCISE 3.13. (i) (Higher-dimensional Cantor grills). Generalize the results of the present subsection to the higher-dimensional Cantor grill $A:=C \times[0,1]^{d}$, where $d$ is an arbitrary positive integer. In particular, show that (with $\mathbf{p}:=2 \pi / \log 3$ and $D_{C}=\log _{3} 2$, as above)

$$
\begin{gathered}
D_{A}=\operatorname{dim}_{B} A=d+\operatorname{dim}_{B} C=d+D_{C} \\
\operatorname{dim}_{P C} A=D_{A}+i \mathbf{p} \mathbb{Z}
\end{gathered}
$$

and

$$
\mathcal{D}_{A}=\mathcal{D}\left(\zeta_{A}\right)=\{0,1, \cdots, d\} \cup \bigcup_{m=0}^{d}\left(\left(m+D_{C}\right)+i \mathbf{p} \mathbb{Z}\right) .
$$

[Hint: In order to establish (3.129) and (3.130), compare $\zeta_{A}(s)$ and $\zeta_{A}(s-d)$ and show that the difference of these two functions is holomorphic in a suitable halfplane, namely,

$$
\left\{\operatorname{Re}(s)>D_{C}+(d-1)\right\} \supseteq\left\{\operatorname{Re}(s) \geq D_{A}\right\} .
$$

The proof of (3.131) is significantly more complicated; if needed, see LapRaZu1, Exple. 2.2.34 and §4.7.1].]

(ii) (Fractal combs). Let $K$ be any compact subset of $\mathbb{R}$ and let $A:=K \times[0,1]^{d}$, with $d \in \mathbb{N}$. Extend the results of part (i) to this more general situation. 
EXERCise 3.14. (Two different Cantor grill RFDs.) Let $A:=C$, the ternary Cantor set, and $\Omega_{1}:=(0,1)^{2}$ while $\Omega_{2}:=(-1,0) \times(0,1)$. Then, show that the complex dimensions of the RFDs $\left(A, \Omega_{1}\right)$ and $\left(A, \Omega_{2}\right)$ are very different. More specifically, as is observed in LapRaZu1, §1.1], it turns out that

$$
\mathcal{D}_{A, \Omega_{1}}=\mathcal{D}_{C \times[0,1]}=\{0,1\} \cup\left(D_{C}+i \mathbf{p} \mathbb{Z}\right) \cup\left(\left(1+D_{C}\right)+i \mathbf{p} \mathbb{Z}\right),
$$

as in (3.128), where $D_{C}=\log _{3} 2$ and $\mathbf{p}=2 \pi / \log 3$ are, respectively, the Minkowski dimension and the oscillatory period of $C$. By contrast,

$$
\mathcal{D}_{A, \Omega_{2}}=\{0,1\} \cup \mathcal{D}_{C}=\{0,1\} \cup\left(D_{C}+i \mathbf{p} \mathbb{Z}\right) .
$$

(Further, all the complex dimensions in either (3.132) or (3.133) are simple.) Thus, the RFD $\left(A, \Omega_{2}\right)$ no longer "sees" the principal complex dimensions of the Cantor grill $C \times[0,1]$ in (3.127) (or of the RFD $\left(A, \Omega_{1}\right)$, according to (3.132)).

In addition to establishing (3.132) and (3.133), provide an intuitive explanation for the striking difference between these two results.

3.4.8. The Cantor dust. Let $A:=C \times C$, where as before, $C$ is the ternary Cantor set. Henceforth, $A$, the Cartesian product of $C$ by itself, is referred to as the Cantor dust. (See LapRaZu1, Fig. 1.2] for a depiction of $A$.) The associated Cantor dust $\operatorname{RFD}(A, \Omega)$ is defined by $\Omega:=(0,1)^{2}$ and $A:=C \times C$, as above.

Then, it is shown in LapRaZu1, Exple. 4.7.15] that $\zeta_{A, \Omega}$ has (for all $\delta>0$ large enough) a meromorphic continuation to all of $\mathbb{C}$ given for every $s \in \mathbb{C}$ by

$$
\zeta_{A, \Omega}(s)=\frac{8}{s\left(3^{s}-4\right)}\left(\frac{J(s)}{6^{s}}+\frac{\Gamma\left(\frac{1-s}{2}\right)}{\Gamma\left(\frac{2-s}{2}\right)} \frac{\sqrt{\pi}}{6^{s} s\left(3^{s}-2\right)}+K(s)\right),
$$

where $\Gamma=\Gamma(s)$ denotes the classic gamma function (which, as we recall, does not have any zeros anywhere in $\mathbb{C}$ but has simple poles at $0,-1,-2,-3, \cdots$. Here, $J(s):=\int_{0}^{\pi / 4}(\cos \theta)^{-s} d \theta$ is an entire function and $K=K(s)$ is a meromorphic function in all of $\mathbb{C}$ with (simple) poles at $1,3,5, \cdots$.

It follows from (3.134) and the aforementioned properties of $\Gamma, J$ and $K$ that

$$
D_{A, \Omega}=D\left(\zeta_{A, \Omega}\right)=\operatorname{dim}_{B}(A, \Omega)=\log _{3} 4=D_{A}=\operatorname{dim}_{B} A,
$$

as expected since $\log _{3} 4=\log _{3} 2+\log _{3} 2=2 \operatorname{dim}_{B} C$, and that the set of complex dimensions (in $\mathbb{C}$ ) of the Cantor dust RFD consists of simple poles of $\zeta_{A, \Omega}$ and is given by

$$
\mathcal{D}_{A, \Omega}=\mathcal{D}\left(\zeta_{A, \Omega}\right)=\{0\} \cup\left(\log _{3} 2+i \frac{2 \pi}{\log 3} \mathbb{Z}\right) \cup\left(\log _{3} 4+i \frac{2 \pi}{\log 3} \mathbb{Z}\right) .
$$

More specifically, due to possible zero-pole cancellations, $\mathcal{D}_{A, \Omega}$ is a subset of the set given on the right-hand side of (3.136) and contains $D_{C}=\log _{3} 2, D_{A}=D_{C \times C}=$ $\log _{3} 4$, as well as at least two nonreal complex conjugate principal complex dimensions. It is conjectured in loc. cit. that, in fact, we have a true equality in (3.136) and hence, in particular, that the set of principal complex dimensions of the RFD $(A, \Omega)$ (as well as of the compact set $A \subseteq \mathbb{R}^{2}$ ) is given by

$$
\operatorname{dim}_{P C}(A, \Omega)=\operatorname{dim}_{P C} A=\log _{3} 4+i \frac{2 \pi}{\log 3} \mathbb{Z}
$$

or is, at least, an infinite subset of the 'periodic set' $\log _{3} 4+i(2 \pi / \log 3) \mathbb{Z}$. Furthermore, the author conjectures entirely similar statements about the set of subcritical complex dimensions; namely, the set of complex dimensions of $(A, \Omega)$ (and of $A$ ) 
should be equal to (or, at least, coincide with an infinite subset of) the periodic set $\log _{3} 2+i(2 \pi / \log 3) \mathbb{Z}$.

We refer to Conjecture 3.16 below for a much more general and precise statement about the complex dimensions (interpreted there in an extended sense) of Cartesian products.

EXERCISE 3.15. (i) Deduce from the above results about the Cantor dust RFD $(A, \Omega)$ analogous results concerning the Cantor dust itself, $A:=C \times C$.

(ii) Generalize the above results about the Cantor dust RFD and the Cantor dust to $A=C^{d}:=C \times \cdots \times C$, the $d$-fold Cartesian product of $C$ with itself (where $d \geq 1$ is arbitrary) and to the associated $\operatorname{RFD}(A, \Omega)$, with $A$ as just above and with $\Omega:=(0,1)^{d}$. We expect that $\operatorname{dim}_{P C} A=\operatorname{dim}_{P C}(A, \Omega)$ and, similarly, $\mathcal{D}_{A}=\mathcal{D}_{A, \Omega}$.

(iii) Finally, replace the ternary Cantor set $C$ by other Cantor-type sets and by more general compact subsets of $\mathbb{R}$ (including lattice and nonlattice self-similar sets).

The following conjecture of the author was motivated, in part, by the results from LapRaZu1 (and LapRaZu2-6]) stated about the complex dimensions of the Cantor grill (in \$3.4.7) and of the Cantor dust (in $\$ 3.4 .8$ ), along with the results of loc. cit. briefly discussed in $\$ 3.3 .4$ about the invariance of the complex dimensions under embeddings into higher-dimensional Euclidean spaces. More specifically, the author was first led to stating this conjecture (in December 2016), on the basis of his joint work on quantized number theory and fractal cohomology (CobLap1-2, Lap10), and especially, due to the requirement that 'fractal cohomology' should satisfy an appropriate analog of the Künneth formula for the cohomology of Cartesian products. (See, especially, Lap10 esp., Chs. 4-6] for more details and motivations; see also 4.1 and 4.4 in the epilogue for a brief discussion of the general context.)

For simplicity, we state the conjecture for compact sets rather than for general RFDs, but clearly, an analogous conjecture can be made about RFDs. We also implicitly assume that the corresponding fractal zeta functions are meromorphic in all of $\mathbb{C} 84$ but we can also state the conjecture relative to a common window (in the sense of Lap-vF4, LapRaZu1]) or more generally, a domain of $\mathbb{C}$ to which the fractal zeta functions can be meromorphically continued 85 Finally, the mathematical notion of divisor (denoted by $\mathfrak{D}(f)$ ) of a meromorphic function $f$ is well known and will be recalled in Definition 3.18 below (following [Lap-vF4, $\S 3.4]$ ). For now, we simply mention that $\mathfrak{D}(f)$ is the multiset of zeros and poles of $f$; that is, the graded set of zeros and poles of $f$, counted according to their multiplicities (with the zeros counted positively and the poles counted negatively).

Conjecture 3.16 (Complex dimensions of Cartesian products, Lap10). Let $A_{1}$ and $A_{2}$ be two bounded (or, equivalently, compact) subsets of $\mathbb{R}^{N_{1}}$ and $\mathbb{R}^{N_{2}}$, respectively. For $j=1,2$, let $\mathfrak{D}\left(A_{j}\right)$ denote the divisor of $\zeta_{A_{j}}, \mathfrak{D}\left(A_{j}\right):=\mathfrak{D}\left(\zeta_{A_{j}}\right)$, and $\mathcal{D}\left(A_{j}\right)=\mathcal{D}\left(\zeta_{A_{j}}\right)$ denote the (multi) set of complex dimensions of $A_{j} 86$

\footnotetext{
${ }^{84} \mathrm{See}$, in particular, Remark 3.17 for a more general situation.

${ }^{85}$ If $f$ and $g$ are two meromorphic functions defined on the same domain $U$ of $\mathbb{C}$, we simply let $\mathfrak{D}(f)=\mathfrak{D}(f ; U)$ and $\mathfrak{D}(g)=\mathfrak{D}(g ; U)$ in the statement of Conjecture 3.16 and with the notation of Definition 3.18

${ }^{86}$ For simplicity, we assume implicitly that $\overline{\operatorname{dim}}_{B} A_{j}<N_{j}$, for $j=1,2$. We also use $\zeta_{A_{j}}$ in order to define both $\mathfrak{D}_{A_{j}}$ and $\mathcal{D}_{A_{j}}$ for $j=1,2$; namely, for $j=1,2, \mathfrak{D}_{A_{j}}:=\mathfrak{D}\left(\zeta_{A_{j}}\right)$ and
} 
Then, we have the identity

$$
\mathfrak{D}\left(A_{1} \times A_{2}\right)=\mathfrak{D}\left(A_{1}\right)+\mathfrak{D}\left(A_{2}\right), 87
$$

Also, we have the inclusion

$$
\mathcal{D}\left(A_{1} \times A_{2}\right) \subseteq \mathcal{D}\left(A_{1}\right)+\mathcal{D}\left(A_{2}\right),
$$

the Minkowski sum of $\mathcal{D}\left(A_{1}\right)$ and $\mathcal{D}\left(A_{2}\right)$. Furthermore, typically (or "generically", in a vague sense), we have an equality in (3.140), because we do not have zero-pole cancellations in such cases:

$$
\mathcal{D}\left(A_{1} \times A_{2}\right)=\mathcal{D}\left(A_{1}\right)+\mathcal{D}\left(A_{2}\right) .
$$

REMARK 3.17. If $\zeta_{A_{1} \times A_{2}}$ is not necessarily meromorphic in all of $\mathbb{C}$ (or in the given domain $U \subseteq \mathbb{C}$ under consideration), but $\zeta_{A_{1}}$ and $\zeta_{A_{2}}$ still are, then, under appropriate hypotheses, we expect (based in part on cohomological and spectral considerations; see 4.3 and 4.4 along with Lap10) that Conjecture 3.16 can be suitably modified and extended by substituting for the ordinary divisor $\zeta_{A_{1} \times A_{2}}$ a "generalized divisor" (still denoted by $\mathcal{D}\left(\zeta_{A_{1} \times A_{2}}\right)$ or, in short, $\left.\mathcal{D}\left(A_{1} \times A_{2}\right)\right)$ which takes into account the (nonremovable) singularities (and not just the poles) of $\zeta_{A_{1} \times A_{2}}$; in that case, we must also replace the Minkowski sum by its closure in the right-hand side of the counterpart of (3.138):

$$
\mathcal{D}\left(A_{1} \times A_{2}\right)=c \ell\left(\mathcal{D}\left(A_{1}\right)+\mathcal{D}\left(A_{2}\right)\right) .
$$

In particular, the counterpart of (3.140) becomes

$$
\mathcal{D}\left(A_{1} \times A_{2}\right) \subseteq c \ell\left(\mathcal{D}\left(A_{1}\right)+\mathcal{D}\left(A_{2}\right)\right) .
$$

Next, as promised, we recall the definition of the divisor of a meromorphic function (see, e.g., Lap-vF4, Def. 3.11]). It goes back at least to Riemann in the related context of Riemann surfaces and has counterparts and various generalizations in many fields, including arithmetic and algebraic geometry, as well as in algebraic combinatorics. The notion of divisor is ideally suited to making precise sense of the possible cancellations between the zeros and poles of a meromorphic function. Hence, its key use in the statement of Conjecture 3.16 above.

Definition 3.18. (Divisor of a meromorphic function). Let $f$ be a meromorphic function on a given domain $U \subset \mathbb{C}$. Then, the divisor of $f$, denoted by $\mathfrak{D}=\mathfrak{D}(f)$, is defined as the formal sum 88

$$
\mathfrak{D}(f)=\mathfrak{D}(f ; U):=\sum_{\omega \in U} \operatorname{ord}(f ; \omega) \cdot \omega,
$$

$\mathcal{D}_{A_{j}}:=\mathcal{D}\left(\zeta_{A_{j}}\right)\left(=\mathcal{D}\left(\widetilde{\zeta}_{A_{j}}\right)\right.$, in this case). [Note that in spite of the functional equation (3.15) (or, more generally, (3.11), in the case of RFDs), $\zeta_{A_{j}}$ and $\widetilde{\zeta}_{A_{j}}$ have the same poles but not necessarily the same zeros.] One could make a similar conjecture without assuming that $\overline{\operatorname{dim}}_{B} A_{j}<N_{j}$ for $j=1,2$ and by using $\widetilde{\zeta}_{A_{j}}$ instead of $\zeta_{A_{j}}$ in order to define both $\mathfrak{D}_{A_{j}}$ and $\mathcal{D}_{A_{j}}$, for $j=1,2$.

${ }^{87}$ Given two subsets (or submultisets) $E_{1}$ and $E_{2}$ of the same additive group, their Minkowski sum $E_{1}+E_{2}$ is defined by

$$
E_{1}+E_{2}=\left\{e_{1}+e_{2}: e_{1} \in E_{1}, e_{2} \in E_{2}\right\}
$$

viewed as a subset (or submultiset) of this same group.

${ }^{88}$ This is an at most countable sum since clearly, $\operatorname{ord}(f, \omega)=0$ whenever $\omega \in U$ is neither a pole nor a zero of $f$. Also, $\mathfrak{D}(f)$ can be viewing as lying in the free ablian group generated by the distinct zeros and poles of $f$. 
where the order of $f$ at $\omega \in U$ is defined as the integer $m \in \mathbb{Z}$ such that the function $\left|f(s)(s-\omega)^{-m}\right|$ is bounded away from 0 and $\infty$ in a neighborhood of $\omega$. Therefore, if $\omega$ is a zero of (positive) multiplicity $m$, then $\operatorname{order}(f, \omega)=m$, a positive integer, whereas if $\omega$ is a pole of $f$ of (positive) multiplicity $n$, then $\operatorname{ord}(f, \omega)=-n=: m$, a negative integer. Furthermore, $\operatorname{ord}(f, \omega)=0$ if $\omega$ is neither a zero nor a pole of $f$ (in $U$ ).

Formally, the Minkowski sum of the divisors $\mathfrak{D}(f)$ and $\mathfrak{D}(g)$ of two meromorphic functions on the same domain $U$ of $\mathbb{C}$ is denoted by $\mathfrak{D}(f)+\mathfrak{D}(g)$ and is given by

$$
\mathfrak{D}(f)+\mathfrak{D}(g)=\sum_{\omega \in U}(\operatorname{ord}(f, \omega)+\operatorname{ord}(g, w)) \cdot \omega .
$$

Note that on the right-hand side of (3.145), some of the coefficients of $\operatorname{ord}(f, \omega)+$ $\operatorname{ord}(g, \omega)$ may vanish, corresponding precisely to the aforementioned (exact) zeropole cancellations.

Remark 3.19. (a) Conjecture 3.16 above is consistent with all the known examples and results, including those described in 3.3 .4 , 3.4.7 and 3.4 .8 (See, in particular, parts $(b)$ and $(c)$ of the present remark for the examples of the Cantor grill and the Cantor dust, respectively.) We warn the interested reader, however, that in the general case, this conjecture is likely to be quite difficult to prove although the coherence and consistency of the fractal cohomology theory partly developed in Lap10 (and briefly discussed in $\$ 4.1$ and $\$ 4.4$ clearly requires that Conjecture 3.16 must be true.

(b) (Cantor grill, revisited) As a simple verification, for the example of the Cantor grill $C \times[0,1]$ discussed in the main text of 33.4 .7 (and corresponding to the case when $d=1$ ) or, more generally, of its higher-dimensional counterpart $A:=C \times[0,1]^{d}$ considered in Exercise $3.13(i)$, we have

$$
\mathcal{D}_{[0,1]^{d}}:=\mathcal{D}\left(\widetilde{\zeta}_{[0,1]^{d}}\right)=\{0,1, \cdots, d\},
$$

as can be easily verified 89 and

$$
\mathcal{D}_{C}=\{0\} \cup \mathcal{D}_{C S}=\{0\} \cup\left(D_{C}+i \mathbf{p} \mathbb{Z}\right),
$$

where $D_{C}=\log _{3} 2$ and $\mathbf{p}=2 \pi / \log 3$. (See (3.17) and (3.20) for a closely related fact.) Therefore, the Minkowski sum $\mathcal{D}_{C}+\mathcal{D}_{[0,1]^{d}}$ is given by

$$
\{0,1, \cdots, d\} \cup\left(\bigcup_{m=0}^{d}\left(\left(m+D_{C}\right)+i \mathbf{p} \mathbb{Z}\right)\right),
$$

which is precisely $\mathcal{D}_{A}=\mathcal{D}_{C \times[0,1]^{d}}$, as given by (3.131) (and, in particular, for the usual Cantor grill corresponding to the choice $d=1$, as given by (3.128)). Of course, this is also in agreement with the result predicted by Conjecture 3.16 in (3.140), and even in (3.141) since we have

$$
\mathcal{D}_{C}+\mathcal{D}_{[0,1]^{d}}=\mathcal{D}_{C \times[0,1]^{d}}
$$

in the present situation, as expected in the "generic" case.

Observe that it is absolutely crucial here that $\mathcal{D}_{C}$ be given by (3.147), as obtained above via the higher-dimensional theory of fractal zeta functions, and not just by $\mathcal{D}\left(\zeta_{C S}\right)=D_{C}+i \mathbf{p} \mathbb{Z}$, as given by the theory of fractal strings and their associated geometric zeta functions. Recall that $\mathcal{D}_{C}=\mathcal{D}\left(\zeta_{C}\right)=\mathcal{D}\left(\widetilde{\zeta}_{C}\right)=$

\footnotetext{
${ }^{89}$ Note that we only use $\widetilde{\zeta}_{[0,1]^{d}}$ here because $D_{[0,1]^{d}}=\operatorname{dim}_{B}[0,1]^{d}=d$.
} 
$\{0\} \cup \mathcal{D}\left(\zeta_{C S}\right)$, where $\mathcal{D}\left(\zeta_{C S}\right)$ is given just above. (An entirely analogous comment can be made about the example of the Cantor dust dealt with in part (c).)

(c) (The Cantor dust, revisited). Let $A=C \times C$ be the Cantor dust, as in 33.4.8. Then, in light of (3.147) in part (b) just above, it is easy to check that $\mathcal{D}_{C}+\mathcal{D}_{C}$ is given by (with the same notation as in part (b) of this remark)

$$
\mathcal{D}_{C}+\mathcal{D}_{C}=\{0\} \cup\left(D_{C}+i \mathbf{p} \mathbb{Z}\right) \cup\left(2 D_{C}+i \mathbf{p} \mathbb{Z}\right),
$$

with $2 D_{C}=\log _{3} 4=D_{A}$, in accord with the result stated in (3.136) and more precisely, immediately after (3.136), and also in agreement with the containment (3.140) of Conjecture 3.16, even though it has not yet been fully proved for this example. In fact, we expect once again that we have an equality in the present situation, as predicted for the "generic" case in (3.141) of Conjecture 3.16 namely, we expect that

$$
\mathcal{D}_{C \times C}=\mathcal{D}_{C}+\mathcal{D}_{C} .
$$

We leave it to the interested reader to calculate the $d$-fold Minkowski sum of $\mathcal{D}_{C}$ and to deduce from it (assuming that we are in the generic case of Conjecture 3.16) the expression for $\mathcal{D}_{C^{d}}$, where $C^{d}$ is the $d$-fold Cartesian product of $C$ by itself, as sought for in part (ii) of Exercise 3.15.

The following example (the Cantor graph RFD) will play an important role in \$3.6 in order to illustrate the definition of fractality in terms of the existence of nonreal complex dimensions.

3.4.9. The devil's staircase (or Cantor graph) RFD. Let $A$ denote the graph of the classic Cantor function, also called from now on the devil's staircase or the Cantor graph. It is well known that $A$ is a self-affine (rather than a self-similar) set in $\mathbb{R}^{2}$; more specifically, it scales distances by the factors $1 / 2$ and $1 / 3$ along the horizontal and vertical directions, respectively. (See, for example, LapRaZu1, Remark 1.2.1] for a more detailed description, along with [Man Plate 83, p. 83] and LapRaZu1, Figs. 1.5-1.7] for an illustration.)

Next, let $\Omega$ be the union of the triangles $\Delta_{k}$ and $\widetilde{\Delta}_{k}$ above and below the horizontal parts of the Cantor graph $A$, for $k=1,2, \cdots$. (For each $k \geq 1$, at the $k$-th stage of the construction of $A$ and of the $\operatorname{RFD}(A, \Omega)$ below, there are $2^{k-1}$ pairs of congruent triangles $\Delta_{k}$ and $\widetilde{\Delta}_{k}$.) Then, $(A, \Omega)$ is called the Cantor graph $R F D$; clearly, it is an RFD in $\mathbb{R}^{2}$. Intuitively, it can be thought of as the Cantor graph viewed from the perspective of the (non-Euclidean) $\ell^{\infty}$ metric of $\mathbb{R}^{2}$, given by $\|(x, y)\|_{\infty}=\max (|x|,|y|)$, for $x, y \in \mathbb{R}^{2}$. In spite of that, $(A, \Omega)$ captures the essence of the Cantor graph.

It is shown in LapRaZu1, Exple. 5.5.14] that $\zeta_{A, \Omega}$ admits a (necessarily unique) meromorphic continuation to all of $\mathbb{C}$, given for every $s \in \mathbb{C}$ by

$$
\zeta_{A, \Omega}(s)=\frac{2}{s\left(3^{s}-2\right)(s-1)} .
$$

It follows that the set of principal complex dimensions of the Cantor graph RFD $(A, \Omega)$ is given by

$$
\operatorname{dim}_{P C}(A, \Omega)=\{1\}
$$

where

$$
D_{A, \Omega}=\operatorname{dim}_{B}(A, \Omega)=1
$$


and that the set of all complex dimensions of $(A, \Omega)$ is

$$
\mathcal{D}_{A, \Omega}=\mathcal{D}\left(\zeta_{A, \Omega}\right)=\{0,1\} \cup\left(\log _{3} 2+i \frac{2 \pi}{\log 3} \mathbb{Z}\right),
$$

with each complex dimension being simple. Apart from the complex dimension at $s=0$, this is in complete agreement with the values of the complex dimensions of the Cantor graph $A$ predicted in Lap-vF2-4 (see, especially, Lap-vF4, §12.1.2]), on the basis of an approximate tube formula for $A$ (but, at the time, without an appropriate definition of higher-dimensional fractal zeta functions).

Furthermore, with $s_{k}:=\log _{3} 2+i(2 \pi / \log 3)$ for each $k \in \mathbb{Z}$, we have that

$$
\operatorname{res}\left(\zeta_{A, \Omega}, s_{k}\right)=\frac{1}{(\log 3)\left(s_{k}-1\right) s_{k}} \text {. }
$$

Moreover,

$$
\operatorname{res}\left(\zeta_{A, \Omega}, 0\right)=\operatorname{res}\left(\zeta_{A, \Omega}, 1\right)=2 .
$$

Let us next briefly consider the Euclidean Cantor graph $R F D\left(A, A_{1 / 3}\right)$, where $A_{1 / 3}$ is the (1/3)-neighborhood (with respect to the Euclidean metric of $\mathbb{R}^{2}$ ) of the Cantor graph $\mathbb{R}^{2}$. Then, it is also shown in LapRaZu1, §5.5.4] that

$$
\mathcal{D}_{A, A_{1 / 3}}=\mathcal{D}\left(\zeta_{A, A_{1 / 3}}\right) \subseteq \mathcal{D}_{(A, \Omega)}=\{0,1\} \cup\left(\log _{3} 2+i \frac{2 \pi}{\log 3} \mathbb{Z}\right)
$$

and that 90

$$
\operatorname{dim}_{P C}\left(A, A_{1 / 3}\right)=\operatorname{dim}_{P C}(A, \Omega)=\{1\}
$$

where

$$
\operatorname{dim}_{B}\left(A, A_{1 / 3}\right)=\operatorname{dim}_{B}(A, \Omega)=D\left(\zeta_{A, \Omega}\right)=D\left(\zeta_{A, A_{1 / 3}}\right)=1 .
$$

It can be checked numerically (see loc. cit.) that $\mathcal{D}_{A, A_{1 / 3}}$ contains several pairs of nonreal complex conjugate complex dimensions with real part $D_{C S}=D_{C}=$ $\log _{3} 2$. In fact, we expect that a lot more is true, as is stated in the following conjecture. (Compare with the inclusion obtained in (3.158).)

Conjecture 3.20. ([LapRaZu4, 7, [LapRaZu1, §5.5.4]). We not only have that $\mathcal{D}_{A, A_{1 / 3}} \subseteq \mathcal{D}_{A, \Omega}$ (as stated in (3.158)) but also that

$$
\mathcal{D}_{A}=\mathcal{D}_{A, A_{1 / 3}}=\mathcal{D}_{A, \Omega},
$$

where (as in (3.155) and with the notation of footnote 90)

$$
\mathcal{D}_{A, \Omega}=\{0,1\} \cup\left(D_{C S}+i \mathbf{p} \mathbb{Z}\right),
$$

with $D_{C S}=\log _{3} 2$ and $\mathbf{p}=(2 \pi / \log 3)$.

\footnotetext{
${ }^{90}$ Observe that we can rewrite (3.158) as follows:

$$
\mathcal{D}_{A, \Omega}=\mathcal{D}_{C S} \cup\{1\},
$$
}

where the set of complex dimensions of the Cantor string $C S$ (and of the set $C$ ) is given by

$$
\mathcal{D}_{C S}=\mathcal{D}_{C}=\{0\} \cup\left(D_{C S}+i \mathbf{p} \mathbb{Z}\right) .
$$

Here, $D_{C S}=D_{C}=\log _{3} 2$ is the Minkowski dimension of the Cantor string (and set) and $\mathbf{p}=$ $(2 \pi / \log 3)$ is its oscillatory period. 
3.4.10. Self-similar sprays. The notion of a self-similar (or, more generally, fractal) spray was introduced in LapPo3, formalizing a number of examples discussed in Lap1, Lap3. It was then used extensively, in particular, in LapPe1-2, LapPeWi1-2 where were established fractal tube formulas extending (and using) those obtained for fractal strings in Lap-vF1-4. (See also, e.g., DemDenKö̈, DemKoÖÜ], DenKoÖÜ] and [KoRati.) The notion of a self-similar spray or RFD introduced in LapRaZu1 (and in LapRaZu4,6) is slightly different from the one used in those references; in particular, its greater flexibility is well suited to a variety of examples discussed in loc. cit. as well as in the present section (e.g., the relative $N$-gasket in 33.4 .4$)$. Since the precise definition is a bit technical, we simply mention that, roughly speaking, a self-similar spray (or RFD) is an $\operatorname{RFD}(A, \Omega)$ in $\mathbb{R}^{N}$ which, up to displacements, is obtained from a single generator (also called the base $R F D)(\partial G, G)$ ) 91 itself an RFD in $\mathbb{R}^{N}$, via a scaling sequence $\mathcal{L}=\left(\ell_{j}\right)$, which is a possibly unbounded self-similar fractal string (in the sense of Lap-vF4, Ch. 3]) with (not necessarily distinct) scaling ratios $r_{1}, \cdots, r_{J}$. Here, $J \geq 2$ and $0<r_{1}, \cdots, r_{J}<1$; furthermore, $\mathcal{L}$ is comprised of all the finite products of elements of the ratio list $\left\{r_{1} \cdots, r_{J}\right\}$.

It follows that $(A, \Omega)$ is the disjoint union of scaled copies of the generating $\operatorname{RFD}(\partial \Omega, \Omega)$, scaled by the self-similar string $\mathcal{L}$. In order for $\Omega$ to have finite total volume, we assume that $G$ is open, $|G|<\infty, \operatorname{dim}_{B}(\partial G, G)<N$ and $\sum_{j=1}^{J} r_{j}^{N}<1$.

Let $(A, \Omega)$ be an arbitrary self-similar spray (or RFD) in $\mathbb{R}^{N}$. It is shown in LapRaZu1, Thm. 4.2.17] that $\zeta_{A, \Omega}$ admits a meromorphic continuation to all of $\mathbb{C}$ given for every $s \in \mathbb{C}$ by

$$
\zeta_{A, \Omega}(s)=\frac{\zeta_{\partial G, G}(s)}{1-\sum_{j=1}^{J} r_{j}^{s}}
$$

or equivalently, by the following factorization formula 92

$$
\zeta_{A, \Omega}(s)=\zeta_{\mathfrak{s}}(s) \cdot \zeta_{(\partial G, G)}(s),
$$

where $\zeta_{\mathbf{s}}(s):=\zeta_{\mathcal{L}}(s)$, the geometric zeta function of the possibly unbounded selfsimilar string $\mathcal{L}$, is called the scaling zeta function of the fractal spray $(A, \Omega)$ and is given (also for every $s \in \mathbb{C}$ ) by

$$
\zeta_{\mathfrak{s}}(s)=\frac{1}{1-\sum_{j=1}^{J} r_{j}^{s}} .
$$

It then follows from (3.165) -(3.167) that (with $\mathcal{D}_{A, \Omega}=\mathcal{D}\left(\zeta_{A, \Omega}\right), \mathcal{D}_{(\partial G, G)}=\mathcal{D}\left(\zeta_{(\partial G, G)}\right)$ and $\left.\mathcal{D}_{\mathfrak{s}}=\mathcal{D}\left(\zeta_{\mathfrak{s}}\right)=\mathcal{D}\left(\zeta_{\mathcal{L}}\right)\right)$

$$
\mathcal{D}\left(\zeta_{A, \Omega}\right) \subseteq \mathcal{D}\left(\zeta_{\mathfrak{s}}\right) \cup \mathcal{D}\left(\zeta_{\partial G, G}\right),
$$

where the containment comes from the fact that there could, in general, be zero-pole cancellations in the expression on the right-hand side of (3.166) (or, equivalently,

\footnotetext{
${ }^{91}$ For the simplicity of exposition, we consider here the case of a single generator. The case of multiple generators (i.e., finitely many generators) is an immediate consequence of the present case of a single generator; see part $(a)$ of Remark 3.21

${ }^{92} \mathrm{~A}$ priori, the equivalent identities (3.165) and (3.166) are valid for $\operatorname{Re}(s)>\bar{D}$, with $\bar{D}=$ $D_{A, \Omega}=D\left(\zeta_{A, \Omega}\right)$, as given by 3.170. However, upon meromorphic continuation, it remains valid in any domain $U$ to which $\zeta_{\partial G, G}$ can be meromorphically extended. For example, we can take $U=\mathbb{C}$ if $G$ is sufficiently "nice" (e.g., monophase or even pluriphase, in the sense of LapPe2, LapPeWi1, and in particular, a nontrivial polytope KoRati.
} 
of (3.165) ). Note that we also have the following equality:

$$
\mathfrak{D}\left(\zeta_{A, \Omega}\right)=\mathfrak{D}\left(\zeta_{\mathfrak{s}}\right) \cup \mathfrak{D}\left(\zeta_{\partial G, G}\right),
$$

where (as in (3.144) $) \mathfrak{D}(f)$ denotes the divisor of the meromorphic function $f$.

We also deduce from (3.166) ) that (with $D_{A, \Omega}=D\left(\zeta_{A, \Omega}\right)$ and $D_{\partial G, G}=D\left(\zeta_{\partial G, G}\right)$ )

$$
D_{A, \Omega}=\max \left(\sigma_{0}, D_{\partial G, G}\right)
$$

or, equivalently,

$$
\overline{\operatorname{dim}}_{B}(A, \Omega)=\max \left(\sigma_{0}, \overline{\operatorname{dim}}_{B}(\partial G, G)\right),
$$

where $\sigma_{0}=D\left(\zeta_{\mathfrak{s}}\right)$ is the similarity dimension of the self-similar spray $(A, \Omega)$ defined as the unique real solution of the Moran equation [Mora]

$$
\sum_{j=1}^{J} r_{j}^{\sigma_{0}}=1
$$

In addition, in light of (3.167), $\mathcal{D}_{\mathfrak{s}}=\mathcal{D}\left(\zeta_{\mathfrak{s}}\right)$ is given as the set of complex solutions of the complexified Moran equation, also called the set of scaling complex dimensions of $(A, \Omega)$ :

$$
\mathcal{D}_{\mathfrak{s}}=\left\{s \in \mathbb{C}: \sum_{j=1}^{J} r_{j}^{s}=1\right\},
$$

so that this latter expression for $\mathcal{D}_{\mathfrak{s}}$ can be substituted in (3.168):

$$
\mathcal{D}_{A, \Omega} \subseteq \mathcal{D}_{\partial G, G} \cup\left\{s \in \mathbb{C}: \sum_{j=1}^{J} r_{j}^{s}=1\right\} .
$$

If there are no zero-pole cancellations in (3.165) (or, equivalently, in (3.166)), which (for "nice" generators) is the case "generically" (in a vague sense), then we have an actual equality in (3.174). (See [LapRaZu1, Thm. 4.2.19].)

If the generator $G$ (a bounded open subset of $\mathbb{R}^{N}$ ) is sufficiently nice (e.g., according to the main result of [KoRati] proving a conjecture in LapPe1-2], if $G$ is a nontrivial polytope, a very frequent situation for the classic self-similar fractals), then it is shown in LapRaZu1, §5.5.6] that

$$
\mathcal{D}_{\partial G, G} \subseteq\{0,1, \cdots, N-1\} .
$$

It therefore follows from (3.169) and (3.174) that

$$
\mathcal{D}_{A, \Omega} \subseteq \mathcal{D}_{\partial G, G} \cup \mathcal{D}_{\mathfrak{s}} \subseteq\{0,1, \cdots N-1\} \cup\left\{s \in \mathbb{C}: \sum_{j=1}^{J} r_{j}^{s}=1\right\},
$$

with equalities in the "generic" case. In such a situation (much as in LapPe1-2), LapPeWi1-2 $), \mathcal{D}_{\partial G, G} \subseteq\{0,1, \cdots, N-1\}$ and $\mathcal{D}_{\mathfrak{s}} \subseteq\left\{s \in \mathbb{C}: \sum_{j=1}^{J} r_{j}^{s}=1\right\}$ are respectively called the integer dimensions and the scaling dimensions of the selfsimilar spray $(A, \Omega)$.

\footnotetext{
${ }^{93}$ Clearly, in light of the definition (3.172) of $\sigma_{0}$, we have $\sigma_{0} \in(0, N)$; indeed, by assumption, $J \geq 2>1$ (hence, $\sigma_{0}>0$ ) and $\overline{\operatorname{dim}}_{B}(\partial G, G)<N$ (hence, $\left.\sigma_{0}<N\right)$. Observe that as a result, and in light of 3.170 and (3.171), we have that $\operatorname{dim}_{B}(A, \Omega)<N$. Thus the complex dimensions of $(A, \Omega)$ can be defined indifferently via $\zeta_{A, \Omega}$ or $\widetilde{\zeta}_{A, \Omega}$.
} 
Sketch of the proof of the factorization formulas (3.165-3.166). It is instructive to provide the proof of formula (3.165) (and hence, equivalently, of the factorization formula (3.166) ), as given in the proof of LapRaZu1, Thms. 4.2.17 and 4.2.19, p. 289].

First, we note that since the (possibly unbounded) self-similar string $\mathcal{L}=$ $\left(\ell_{j}\right)_{j \geq 1}$ has for 'scales' $\left\{\ell_{j}: j \geq 1\right\}$, the free monoid with generators $r_{1}, \cdots, r_{J}$, $\mathcal{L}$ satisfies the following self-similar identity (much as in Lap-vF4, §4.4.1]) 94

$$
\mathcal{L}=\mathcal{L}_{0} \sqcup \bigsqcup_{j=1}^{J}\left(r_{j} \mathcal{L}\right), \text { where } \mathcal{L}_{0}:=\{1\} .
$$

Further, a moment's reflection shows that we can deduce from (3.177) the following self-similar identity satisfied by the self-similar spray $(A, \Omega)$ :

$$
(A, \Omega)=(\partial G, G) \sqcup \bigsqcup_{j=1}^{J}\left(r_{j}(A, \Omega)\right),
$$

where much as in (3.177), the symbol $\sqcup$ denotes the disjoint union of RFDs in $\mathbb{R}^{N}$. (See LapRaZu1, Def. 4.1.43] for the precise definition of such a disjoint union of RFDs in $\mathbb{R}^{N}$, which is itself an RFD in $\mathbb{R}^{N}$.)

Now, by combining the scaling (and the invariance) property of the distance zeta function (see $\$ 3.3 .3$ and $\$ 3.3 .4$ according to which (for all $s \in \mathbb{C}$ )

$$
\zeta_{r_{j}(A, \Omega)}(s)=r_{j}^{s} \zeta_{A, \Omega}(s) \text {, for each } j=1, \cdots, J,
$$

where $r_{j}(A, \Omega):=\left(r_{j} A, r_{j} \Omega\right)$, along with the (finite) additivity of the distance zeta function under disjoint unions (see a special case of [LapRaZu1, Prop. 4.1.17]) 95 we obtain the following functional equation,

$$
\zeta_{A, \Omega}(s)=\zeta_{\partial G, G}(s)+\sum_{j=1}^{J} r_{j}^{s} \zeta_{A, \Omega}(s),
$$

which is equivalent to (3.165) (and to (3.166)), after an elementary factorization.

REMARK 3.21. (a) (Multiple generators). In the case of a self-similar spray $(A, \Omega)$ with multiple generators, $G_{1}, \cdots, G_{Q}$, we simply add up the results obtained for each of the generators. More specifically, in light of (3.165)-(3.167) applied to each of the generators, we then have that

$$
\zeta_{A, \Omega}(s)=\frac{\sum_{q=1}^{Q} \zeta_{\partial G_{q}, G_{q}}(s)}{1-\sum_{j=1}^{J} r_{j}^{s}} .
$$

(b) (Fractal sprays). For (not necessarily self-similar) fractal sprays, several (but not all) of the above results are still valid. More specifically, if $(A, \Omega)$ is a fractal spray RFD in $\mathbb{R}^{N}$, with a single generator $(\partial G, G)$ scaled by the (not necessarily bounded or self-similar) fractal string $\mathcal{L}=\left(\ell_{j}\right)_{j \geq 1}$ and such that $|\Omega|<\infty$ and $\overline{\operatorname{dim}}_{B}(\partial G, G)<N$, then

$$
\bar{D}=\overline{\operatorname{dim}}_{B}(A, \Omega)=\max \left(\bar{D}_{\mathfrak{s}}, \overline{\operatorname{dim}}_{B}(\partial G, G)\right),
$$

\footnotetext{
${ }^{94}$ Here, in (3.177), the symbol $\sqcup$ denotes the disjoint union of fractal strings.

${ }^{95}$ This finite additivity property can be easily established. The significantly more delicate countable additivity property (precisely stated and established in LapRaZu1, Prop. 4.1.17]) is not needed here but is frequently used throughout LapRaZu1.
} 
where $D_{\mathfrak{s}}:=D\left(\zeta_{\mathcal{L}}\right)=D\left(\zeta_{\mathfrak{s}}\right), \bar{D}:=D\left(\zeta_{A, \Omega}\right)$ and $\bar{D}_{\partial G, G}:=D\left(\zeta_{\partial G, G}\right)=\overline{\operatorname{dim}}_{B}(\partial G, G)$.

Then, for all $s \in \mathbb{C}$ such that $\operatorname{Re}(s)>\bar{D}$ (and, hence, upon meromorphic continuation and for $G$ nice enough 96 for all $s \in \mathbb{C}$ ),

$$
\zeta_{A, \Omega}(s)=\zeta_{\mathfrak{s}}(s) \cdot \zeta_{\partial G, G}(s),
$$

where $\zeta_{\mathfrak{s}}:=\zeta_{\mathcal{L}}$ denotes the meromorphic continuation of $\zeta_{\mathcal{L}}$ :

$$
\zeta_{\mathfrak{s}}(s):=\zeta_{\mathcal{L}}(s)\left(=\sum_{j \geq 1} \ell_{j}^{s}, \text { for } \operatorname{Re}(s)>\bar{D}_{\mathfrak{s}}\right) .
$$

It then follows from (3.183) that (with $\mathcal{D}_{\mathfrak{s}}:=\mathcal{D}\left(\zeta_{\mathcal{L}}\right)=\mathcal{D}\left(\zeta_{\mathfrak{s}}\right)$ )

$$
\mathcal{D}_{A, \Omega} \subseteq \mathcal{D}_{\partial G, G} \cup \mathcal{D}_{\mathfrak{s}},
$$

with equality instead of an inclusion, unless there are zero-poles cancellations. Furthermore, we always have the following identity between divisors:

$$
\mathfrak{D}_{A, \Omega}=\mathfrak{D}_{\partial G, G} \cup \mathfrak{D}_{\mathfrak{s}} .
$$

Naturally, a comment entirely similar to the one made in part (a) of this remark applies here if the fractal spray has multiple (i.e., finitely many) generators instead of a single generator.

In the next exercise, the interested reader is asked implicitly to use, in particular, the results of the present subsection in order to answer the various questions.

EXERCise 3.22. (a) (Sierpinski gasket). Let $A$ be the classic Sierpinski gasket. Then, calculate $\zeta_{A}, \zeta_{A, T}$ (where $T$ is the equilateral triangle with side lengths 1 ), and deduce from this computation the two sets of complex dimensions $\mathcal{D}_{A}$ and $\mathcal{D}_{A, T}$, respectively. Compare your results with those stated in 3.4 .1 (or with the $N=2$ case of 3.4.4).

(b) (Sierpinski carpet). Let $A$ be the classic Sierpinski carpet. Then, answer the same questions as in part (a) just above, except with the unit triangle $T$ replaced by the open unit square $S:=(0,1)^{2}$, and with 33.4 .1 and 33.4 .4 replaced by 93.4 .2 and 3.4 .3 respectively.

(c) Answer analogous questions for the relative (or inhomogeneous) $\mathrm{N}$-gasket, as discussed in 3.4.4 as well as for the $\frac{1}{2}$-square and $\frac{1}{3}$-square fractals, as discussed in 3.4 .5 .

We refer the interested reader to LapRaZu1-10 for many other examples of computations of fractal zeta functions and complex dimensions (as well as of fractal tube formulas, in regard to 3.5 just below). These examples include non selfsimilar fractals or RFDs such as fractal nests (see LapRaZu1, §3.5 and Exples. 5.5.16 and 5.5.24 in \$5.5.5]) as well as bounded and unbounded geometric chirps (see LapRaZu1, §3.6, §4.4.1 and Exple. 5.5.19 in §5.5.5]). They also include examples of fractal strings and RFDs, as well as compact sets, with principal complex dimensions having arbitrarily prescribed (finite or infinite) multiplicities (see LapRaZu1, Thms. 3.3.6 and 4.2.19]).

\footnotetext{
${ }^{96}$ This is the case, for example, if $G$ is a nontrivial polytope KoRati or more generally, if $G$ is either monophase or pluriphase (in the sense of [LapPe2, LapPeWi1]).
} 
3.5. Fractal tube formulas and Minkowski measurability criteria: Theory and examples. The goal of this subsection is to briefly state (in 3.5.1) the higher-dimensional analog (obtained in LapRaZu6 and LapRaZu1, §§5.1-5.3]) of the fractal tube formulas obtained for fractal strings in Lap-vF2-4 (see, especially, Lap-vF4, Ch. 8]). In fact, the latter tube formulas (briefly discussed in 92.1) are now but a very special case of their higher-dimensional counterparts 97

We will also state (in 3.5 .2 ) some of the Minkowski measurability criteria obtained in LapRaZu1, §5.4.3 and §5.4.4].

In fact, the main goal of this section is to illustrate (in 33.5.3) the aforementioned results concerning fractal tube formulas and Minkowski measurability criteria (and established, in particular, in LapRaZu7 and LapRaZu1, §5.4]), in 3.5 .1 and 3.5 .2 , respectively) via a variety of examples, many of which have been discussed from other points of view earlier in the paper, especially in 3.4

3.5.1. Fractal tube formulas for RFDs in $\mathbb{R}^{N}$, via distance and tube zeta functions. Recall from 2.1, adapted to the present much more general situation of RFDs in $\mathbb{R}^{N}$, that a fractal tube formula enables us to express the tube function

$$
\varepsilon \mapsto V(\varepsilon)=V_{A, \Omega}(\varepsilon):=\left|A_{\varepsilon} \cap \Omega\right|_{N}
$$

of a $\operatorname{RFD}(A, \Omega)$ in $\mathbb{R}^{N}$ in terms of the complex dimensions of $(A, \Omega)$ and the associated residues of the corresponding fractal zeta function (here, either the distance or the tube zeta function of $(A, \Omega)$ ).

An important feature of the higher-dimensional theory of complex dimensions is that such fractal tube formulas can be established under great generality for RFDs in $\mathbb{R}^{N}$, via either distance zeta functions or tube zeta functions, as well as via other fractal zeta functions, such as the so-called shell zeta functions and the Mellin zeta functions, which are of interest in their own right but are also used in LapRaZu1, Ch. 5] in key steps towards the proof of the fractal tube formulas and of related Minkowski measurability criteria. We will limit ourselves here to the case of distance and tube zeta functions.

Depending on the growth assumptions made about the fractal zeta functions under consideration 98 we obtain fractal tube formulas with error term or without error term (i.e., exact), as well as interpreted either pointwise or distributionally. Therefore, just as in the one-dimensional case of fractal strings, but now in the much more general case of RFDs (and, in particular, of bounded sets) in $\mathbb{R}^{N}$, there is a lot of flexibility for obtaining and applying such tube formulas.

Let us now be a little bit more specific, while avoiding technicalities and cumbersome (although useful) definitions. We state all of the results for RFDs $(A, \Omega)$ in $\mathbb{R}^{N}$ but, as usual, the special case of bounded subsets $A$ of $\mathbb{R}^{N}$ is obtained by

${ }^{97}$ However, as is often the case in such situations, the results and techniques developed in Lap-vF4, Chs. 5 and 8] in order to prove fractal tube formulas and other explicit formulas for (generalized) fractal strings are key to establishing the higher-dimensional (pointwise and distributional, exact or with error term) fractal tube formulas. Nevertheless, a significant amount of additonal work is required in order to prove those tube formulas for RFDs (or, in particular, bounded sets) in $\mathbb{R}^{N}$; see, especially, LapRaZu1, $\left.\S \S 5.1-5.3\right]$.

${ }^{98}$ These polynomial-type growth conditions are referred to (much as in Lap-vF4 Chs. 5 and 8]) as languidity conditions in the case of tube formulas with error term and as strong languidity conditions in the case of exact tube formulas. These conditions are slightly different for distance and tube zeta functions; see LapRaZu1 Ch. 5] for more details. 
simply considering the associated $\operatorname{RFD}\left(A, A_{\delta_{1}}\right)$ for some fixed $\delta_{1}>0$; as before, which $\delta_{1}>0$ is chosen turns out to be unimportant.

We begin by (loosely) stating the fractal tube formula via distance zeta functions. Let $(A, \Omega)$ be a RFD in $\mathbb{R}^{N}$ such that $\bar{D}=\overline{\operatorname{dim}}_{B}(A, \Omega)<N$. Then, if $(A, \Omega)$ is $d$-languid (which roughly means that the distance zeta function satisfies some mild polynomial growth conditions), we have the following fractal formula, expressed via $\zeta_{A, \Omega} 99$

$$
V_{A, \Omega}(\varepsilon)=\sum_{\omega \in \mathcal{D}_{A, \Omega}} c_{\omega} \frac{\varepsilon^{N-\omega}}{N-\omega}+R(\varepsilon),
$$

where $\mathcal{D}_{A, \Omega}=\mathcal{D}_{A, \Omega}(U)$ is the set of visible complex dimensions of $(A, \Omega)$ (defined as the poles of $\zeta_{A, \Omega}$ belonging to $U$ ) and $U$ is a suitable domain of $\mathbb{C}$ (to which $\zeta_{A, \Omega}$ can be meromorphically extended to a $d$-languid function). Furthermore, for each $\omega \in \mathcal{D}_{A, \Omega}$,

$$
c_{\omega}:=\operatorname{res}\left(\zeta_{A}, \omega\right)
$$

so that (3.188) can be rewritten equivalently in the following form (still in the case of simple complex dimensions):

$$
V_{A, \Omega}(\varepsilon)=\sum_{\omega \in \mathcal{D}_{A, \Omega}} \operatorname{res}\left(\zeta_{A, \Omega}, \omega\right) \frac{\varepsilon^{N-\omega}}{N-\omega}+R(\varepsilon) .
$$

Moreover, in (3.188) and (3.190), $R(\varepsilon)$ is an error tem (of lower order than the sum over the complex dimensions) which can be estimated explicitly 100

If, in addition, $(A, \Omega)$ (i.e., $\left.\zeta_{A, \Omega}\right)$ is strongly $d$-languid (a growth condition which requires that $U:=\mathbb{C}$ and is stronger than $d$-languidity), then $R(\varepsilon) \equiv 0$ in (3.188) (and equivalently, in (3.190) or in (3.192)). Hence, we obtain an exact fractal tube formula in this case (i.e., a tube formula without error term):

$$
V_{A, \Omega}(\varepsilon)=\sum_{\omega \in \mathcal{D}_{A, \Omega}} \operatorname{res}\left(\zeta_{A, \Omega}, \omega\right) \frac{\varepsilon^{N-\omega}}{N-\omega} .
$$

Finally, we note that if the visible complex dimensions are not necessarily all simple, then (for example) the tube formula (3.190) takes the following, slightly more complicated, form (except for this modification, all the other statements and hypotheses are identical, otherwise) 101

$$
V_{A, \Omega}(\varepsilon)=\sum_{\omega \in \mathcal{D}_{A, \Omega}} \operatorname{res}\left(\frac{\varepsilon^{N-s}}{N-s} \zeta_{A, \Omega}(s), \omega\right)+R(\varepsilon),
$$

with $R(\varepsilon) \equiv 0$ in the case of an exact formula.

Entirely analogous fractal tube formulas are also obtained via the tube (instead of the distance) zeta function; that is, for $\widetilde{\zeta}_{A, \Omega}$ instead of $\zeta_{A, \Omega}$. The main difference is that in the counterparts of the fractal tube formulas (3.188) and (3.190)-(3.192), we must replace $\varepsilon^{N-\omega} /(N-\omega)$ by $\varepsilon^{N-\omega}$, while in the counterpart of (3.192), we

\footnotetext{
${ }^{99}$ For the simplicity of exposition, we assume at first that all of the (visible) complex dimensions (i.e., the visible poles of $\zeta_{A, \Omega}$ ) are simple; see (3.192) for the general case when some (or possibly all) of the complex dimensions are multiple.

${ }^{100}$ Naturally, in the case of a pointwise (respectively, distributional) tube formula, $R(\varepsilon)$ is a pointwise (respectively, distributional) error term.

${ }^{101}$ Here, for clarity, we write $\operatorname{res}(f(s), \omega)$ (instead of $\left.\operatorname{res}(f, \omega)\right)$ to denote the residue of a meromorphic function $f=f(s)$ at $s=\omega$.
} 
must replace $\frac{\varepsilon^{\omega-s}}{N-s} \zeta_{A, \Omega}(s)$ by $\varepsilon^{N-s} \zeta_{A, \Omega}(s)$. The only other difference, truly minor this time, is that the $d$-languidity (respectively, strong $d$-languidity) condition must be replaced by the slightly different languidity (respectively, strong languidity) condition, in the case of a (pointwise or distributional) fractal tube formula with (respectively, without) error term.

For example, the (pointwise or distributional) fractal tube formula, expressed via the tube zeta function $\widetilde{\zeta}_{A, \Omega}$, takes the following form (in the case of simple complex dimensions) 102

$$
V_{A, \Omega}(\varepsilon)=\sum_{\omega \in \mathcal{D}_{A, \Omega}} \operatorname{res}\left(\widetilde{\zeta}_{A, \Omega}, \omega\right) \varepsilon^{N-\omega}+R(\varepsilon) .
$$

In addition, if $(A, \Omega)$ (i.e., $\left.\widetilde{\zeta}_{A, \Omega}\right)$ is strongly languid (which requires that $U:=\mathbb{C}$ ), then we can let $R(\varepsilon) \equiv 0$ in (3.193); that is, we obtain an exact fractal tube formula in this case.

REMARK 3.23. (a) For the precise statements and the full proofs of the above (as well as of other pointwise and distributional) fractal tube formulas, we refer the interested reader to LapRaZu6 or LapRaZu1, §§5.1-5.3].

(b) Much as was discussed in 2.5 (see, especially, Remark 2.7), and as is apparent in (3.188), (3.190), (3.191) and (3.193), each (visible) simple complex dimension $\omega \in \mathcal{D}_{A, \Omega}$ gives rise to an oscillatory term, proportional to $\varepsilon^{N-\omega}$, with exponent the fractal complex co-dimension $N-\omega$ of $\omega 103$ (Naturally, for each individual complex dimension, these oscillations are multiplicatively periodic. In order to obtain the associated additive or ordinary oscillations, it suffices to let $x:=$ $\log \left(\varepsilon^{-1}\right)$.) Furthermore, as was mentioned before in 2 in the case of fractal strings, as $\omega$ varies in $\mathcal{D}_{A, \Omega}$, the amplitudes (respectively, frequencies) of these oscillations are governed by the real (respectively, imaginary) parts of the complex dimensions. Observe that in the case of simple poles (as in (3.188), (3.190)-(3.191) and (3.193) in 3.5.1), the (typically) infinite sum over the complex dimensions of the RFD $(A, \Omega)$ can be thought of as (pointwise or distributional) natural generalizations of Fourier series or of almost periodic functions (or distributions). (Compare with Schw, §VII, I] and [Boh], respectively.)

We close this subsection by placing the above results in a broader context and providing related references in geometric measure theory and convex geometry. Prior to that, let us recall that the first fractal tube formulas (expressed in terms of complex dimensions and geometric zeta functions) were obtained in Lap-vF1-4 (see, especially, Lap-vF4, Ch. 8]), in the case of fractal strings. In the case of fractal sprays (roughly, higher-dimensional analogs of fractal strings), fractal tube formulas were obtained in LapPe2-3] and, in greater generality, in LapPeWi1, but without a natural notion of associated fractal zeta function. Finally, the fractal tube formulas described in the present subsection (i.e., 3.5.1) were obtained in LapRaZu6 and LapRaZu1, Ch. 5]. They are expressed in terms of complex dimensions and natural fractal zeta functions and include the earlier fractal tube

\footnotetext{
${ }^{102}$ Compare with its counterpart, 3.190, expressed via the distance zeta function $\zeta_{A, \Omega}$.

${ }^{103}$ In the case when $\omega$ is a multiple complex dimension, this oscillatory term is modulated by the multiplication by a suitable polynomial in the variable $x:=\log \left(\varepsilon^{-1}\right)$ and of degree equal to the multiplicity minus one.
} 
formulas for fractal strings from Lap-vF1-4 and for fractal sprays LapPe2-3, LapPeWi1; see, in particular, Example 3.26 and Example 3.36.

REMARK 3.24. (Tube formulas and curvatures: Brief history, references, and beyond.) It is well known that for a (nonempty) compact convex set $A$ of $\mathbb{R}^{N}$, the tube function $V_{A}(\varepsilon):=\left|A_{\varepsilon}\right|_{N}$ is a polynomial of degree (at most) $N$ in $\varepsilon$, the coefficients of which can be interpreted geometrically. This is known as Steiner's formula (Stein, 1840). More specifically,

$$
V_{A}(\varepsilon)=\sum_{\alpha=0}^{N} \gamma_{\alpha} \mu_{\alpha}(A) t^{N-\alpha},
$$

where for each $\alpha \in\{0,1, \cdots, N\}, \gamma_{\alpha}$ is the volume of the unit ball in $\mathbb{R}^{N-\alpha}$ and the (normalized) coefficients $\mu_{\alpha}(A)$ have either a geometric, combinatorial or algebraic interpretation. (See, e.g., $\mathbf{S c h m}$ and [KlRot.) For example, $\mu_{N}(A)=|A|_{N}$ is the volume of $A, \mu_{N-1}(A)$ is the surface area of $A, \cdots, \mu_{1}(A)$ is its mean width, while $\mu_{0}(A)$ is its Euler characteristic (equal to 1 in the present case, but equal to any integer in more general situations).

Furthermore, H. Weyl (Wey3 , 1939) has obtained an analog of Steiner's formula when $A$ is a smooth compact submanifold of Euclidean space $\mathbb{R}^{N}$, thereby interpreting the coefficients $\mu_{\alpha}(A)$ as suitable curvatures, now called the Weyl curvatures of $A$ (see, e.g., BergGos and Gra]).

Moreover, H. Federer ([Fed1], 1969) has unified and extended both Steiner's formula and Weyl's tube formula by establishing their counterpart for sets of positive reach 104 In the process, he introduced (real or signed) measures $\mu_{\alpha}$ (for $\alpha \in\{0,1, \cdots, N\}$ ), now called Federer's curvature measures, whose total mass $\mu_{\alpha}(A)$ coincide with the normalized coefficients of (3.194). He also obtained a localized version of the tube formula (3.194), expressed in terms of the values of the measures $\mu_{\alpha}$, for $\alpha \in\{0,1, \cdots, N\}$, at suitable Borel subsets of $\mathbb{R}^{N}$ (or of $\left.\mathbb{R}^{N} \times S^{N-1}\right)$.

Much later, 'fractal curvatures' were introduced by S. Winter in Wi , M. Zähle [Z̈̈3-4, and S. Winter and M. Zähle [WiZä], for certain classes of self-similar deterministic or random fractals, but still for integer values of the index. The author has long conjectured that there should exist suitable notions of complex

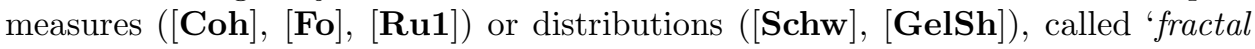
curvature measures', associated with each (visible) complex dimension $\omega \in \mathcal{D}_{A}$ and enabling us to interpret geometrically the (normalized) coefficients of the fractal tube formulas (see, for example, (3.188) and (3.1931). Also, a local fractal tube formula, yet to be precisely formulated and to be rigorously established, should extend Federer's local tube formula, and be expressed (in the case of simple poles) in terms of the residues of a suitably defined local fractal zeta function evaluated at each of the visible complex dimensions. (See LapRaZu1, Pb. 6.2.3.8 and App. B]. 105

${ }^{104} \mathrm{~A}$ (compact) subset $A$ of $\mathbb{R}^{N}$ is said to be of positive reach if there exists $\eta \in(0,+\infty]$ such that each point of $A_{\eta}$, the $\eta$-neighborhood of $A$, has a unique metric projection onto $A$. Clearly, a convex set has infinite reach.

${ }^{105}$ See also the earlier work in LapPe2-3 and LapPeWi1 for the very special case of fractal sprays, as well as LapPe1 which dealt with the example of the Koch snowflake curve (but without the use of any zeta functions). 
We close this discussion of tube formulas by mentioning several relevant references (beside [Stein], Mink], Wey3 and [Fed1]), including the books [Fed2], KlRot, BergGos, Gra, and [Schn, along with the papers HugLasWeil, KeKom, Kom, LapPe1-3, LapPeWi1, LapLu-vF1-2, LapRaZu6, Ol1-2], [Sta, Wi], WiZä], Za1-4, as well as [Lap-vF4, §13.1] and LapRaZu1, Ch. $5]$, and the many relevant references therein.

3.5.2. Minkowski measurability criteria for RFDs in $\mathbb{R}^{N}$. In this subsection, we briefly discuss, in particular, a few of the Minkowski measurability criteria obtained in LapRaZu5,7 or in LapRaZu1, §5.4], to which we refer for further information and for closely related necessary or sufficient conditions for Minkowski measurablity. The criteria will be stated for RFDs in $\mathbb{R}^{N}$, but as usual, can also be applied to bounded subsets (which, as we know by now, are special cases of RFDs).

The main criterion (see LapRaZu1, Thm. 5.4.20]) is expressed in terms of the distance zeta function:

Let $(A, \Omega)$ be a RFD in $\mathbb{R}^{N}$. Assume that $D:=\operatorname{dim}_{B} A$ exists and $D<N$. Then, under suitable hypotheses 106 the following statements are equivalent:

(i) The RFD $(A, \Omega)$ is Minkowski measurable.

(ii) The Minkowski dimension $D$ is the only principal complex dimension of $(A, \Omega)$ (i.e., the only pole of $\zeta_{A, \Omega}$ with real part equal to $\left.D\right)$ and it is simple 107

The above Minkowski measurability criterion is the higher-dimensional counterpart of Theorem 2.2 in 92.1 , the Minkowski measurability criterion for fractal strings obtained in Lap-vF4 §8.3]. Its proof involves several key ingredients including, especially, the Wiener-Pitt Tauberian theorem PitWie] (stated, e.g., in Pit, Kor and LapRaZu1, Thm. 5.4.1]), a version of the fractal tube formula (with error term) via $\zeta_{A, \Omega}$ discussed in $\$ 3.5 .1$ just above, as well as a uniqueness theorem for almost periodic functions (or rather, distributions [Schw, §VI.9.6]).

An analog for the tube (rather than the distance) zeta function $\widetilde{\zeta}_{A, \Omega}$ of the above Minkowski measurability criterion for RFDs is also obtained in LapRaZu1, Thm. 5.4.25].

Moreover, a necessary (respectively, sufficient) condition for the Minkowski measurability of RFDs is obtained in LapRaZu1, Thm. 5.4.15] (respectively, LapRaZu1, Thm. 5.4.2]).

In addition, the case of RFDs for which the underlying scaling law is no longer a power law but is governed instead by a nontrivial gauge function $h=h(t)$ (with $t \in(0,1)$, say) 108 is examined in LapRaZu6-7]; see also [LapRaZu4) and LapRaZu1, \$5.4.4] where both an $h$-Minkowski measurability criterion and an

${ }^{106}$ Namely, $(A, \Omega)$ (i.e., $\zeta_{A, \Omega}$ ) is assumed to be $d$-languid (as in 3.5 .1 for a screen $S$ passing between the critical line $\{\operatorname{Re}(s)=D\}$ and all of the complex dimensions of $(A, \Omega)$ with real part $<D$. (Roughly speaking, a screen $S$ is a suitable curve bounding the region $U$, with $\bar{U} \supseteq\{\operatorname{Re}(s)=$ $D\}$, to which $\zeta_{A, \Omega}$ is meromorphically continued, and extending to infinity in the vertical direction. Also, $S$ is required not to contain any pole of $\zeta_{A, \Omega}$; see [Lap-vF4, §5.3] or [LapRaZu1, §5.1.1].) simple.

${ }^{107}$ Equivalently, the $\operatorname{RFD}(A, \Omega)$ does not have any nonreal complex dimension, and $D$ is

${ }^{108}$ The standard case when the underlying scaling law is a power law corresponds to the trivial gauge function $h(t) \equiv 1$. 
optimal $h$-fractal tube expansion are obtained, especially for gauge functions of the form $h(t):=\left(\log t^{-1}\right)^{m-1}$ for some integer $m \geq 2$ (corresponding, e.g., under appropriate hypotheses, to $D=\operatorname{dim}_{B}(A, \Omega)$ being a multiple pole of order $m$.

In the definition of the (upper, lower) Minkowski content, relative to a given gauge function $h$, where for some $\varepsilon_{0}>0, h:\left(0, \varepsilon_{0}\right) \rightarrow(0,+\infty)$ is a function of slow growth satisfying suitable conditions near 0 (including the fact that $h(t) \rightarrow+\infty$ as $\left.t \rightarrow 0^{+}\right)$, one simply replaces $\varepsilon^{N-D}$ by $\varepsilon^{N-D} h(\varepsilon)$. For example, assuming that $D=\operatorname{dim}_{B}(A, \Omega)$ exists, the upper $h$-Minkowski content of $(A, \Omega)$ is given by

$$
\mathcal{M}^{*}((A, \Omega), h):=\varlimsup_{\varepsilon \rightarrow 0^{+}} \frac{V_{A, \Omega}(\varepsilon)}{\varepsilon^{N-D} h(\varepsilon)},
$$

and similarly for the lower $h$-Minkowski content, $\mathcal{M}_{*}((A, \Omega), h)$, and the $h$-Minkowski content, $\mathcal{M}(A, \Omega, h)$. (See HeLap and LapRaZu1, Eq. (4.5.10), p. 352, and $\S 6.1 .1 .2$, pp. 544-545].) Examples of allowable gauge functions considered in HeLap, LapRaZu1, LapRaZu7 and LapRaZu10 include $\log ^{k}\left(t^{-1}\right)$, for all $t \in(0,1)$, where $k \in \mathbb{N}$ is arbitrary and $\log ^{k}$ denotes the $k$-th iterated logarithm. The reciprocals $1 / h(t)$ of allowable gauge functions are also considered in the above definitions and references.

Although we do not wish to go into the technical details here, we mention that in LapRaZu10, connections between generalized Minkowski contents with logarithm-type gauge functions, Minkowski measurability criteria, and appropriate Riemann surfaces (see, e.g., [Ebe, [Schl]), are explored. These types of generalized Minkowski contents arise naturally in certain geometric situations and in the study of certain dynamical systems.

REMARK 3.25. (Extension to Ahlfors metric spaces.) It is noteworthy that essentially the entire (higher-dimensional) theory of complex dimensions, fractal zeta functions and fractal tube formulas (including the Minkowski measurability criteria) from LapRaZu1 and the accompanying series of papers can be extended without change to a large class of metric measure spaces (see, e.g., DaMcCS] called Ahlfors spaces, doubling spaces or else, spaces of homogeneous type, and of frequent use in harmonic analysis, nonsmooth analysis, fractal geometry and the theory of dynamical systems 109 This extension is carried out in Wat and LapWat and the corresponding theory is illustrated by several examples of computation of complex dimensions and concrete fractal tube formulas, both in the setting of Ahlfors spaces and a little beyond.

3.5.3. Examples. In the present subsection, we illustrate by means of a variety of examples some of the results about fractal tube formulas and Minkowski measurability criteria discussed in 33.5 .1 and 3.5 .2 respectively. In the process, in order to avoid unnecessary repetitions, we often refer to the corresponding examples in §3.4.

EXAMPLE 3.26. (Fractal strings). We briefly explain here how to recover the fractal tube formulas for fractal strings from Lap-vF4]) discussed in $\$ 2.1$ above.

${ }^{109} \mathrm{~A}$ metric space $(X, \mathbf{d})$ of finite diameter and equipped with a positive Borel measure $\mu$ (i.e., a metric measure space) is said to be an Ahlfors space of Ahlfors dimension $\alpha$ if $\mu\left(B_{r}(x)\right)$ is comparable to $r^{\alpha}$, where $B_{r}(x)$ is the closed ball (with respect to the metric $\mathbf{d}$ ) of center $x$ and radius $r$, and with $x \in X$ arbitrary. (The implicit constants must, of course, be independent of $x \in X$ and of $r>0$ sufficiently small.) In this case, $\alpha$ coincides with the Hausdorff and Minkowski dimensions of $X$. 
Let $\mathcal{L}=\left(\ell_{j}\right)_{j \geq 1}$ be a (bounded) fractal string and let $\Omega \subseteq \mathbb{R}$ be any geometric realization of $\mathcal{L}$ as an open set with finite length (i.e., $|\Omega|_{1}<\infty$ ). Furthermore, let $(\partial \Omega, \Omega)$ be the associated RFD in $\mathbb{R}$. Then, as we have seen in 3.2 .2 , the distance zeta function $\zeta_{\partial \Omega, \Omega}$ of the RFD $(\partial \Omega, \Omega)$ and the geometric zeta function $\zeta_{\mathcal{L}}$ of the fractal string $\mathcal{L}$ are connected via the following functional equation:

$$
\zeta_{\partial \Omega, \Omega}(s)=\frac{2^{1-s}}{s} \zeta_{\mathcal{L}}(s)
$$

for all $s \in U$, where $U$ is any domain of $\mathbb{C}$ to which $\zeta_{\mathcal{L}}$ (or, equivalently, $\zeta_{\partial \Omega, \Omega}$ ) can be meromorphically continued. As a result, provided $0 \in U$ (and assuming for simplicity that $\left.\zeta_{\mathcal{L}}(0) \neq 0\right)$, then

$$
\mathcal{D}_{\partial \Omega, \Omega}=\mathcal{D}_{\mathcal{L}} \cup\{0\}
$$

where the union is taken between multisets, as usual. More specifically, if 0 is a pole of $\zeta_{\mathcal{L}}$ of multiplicity $m \geq 0$ (the case when $m=0$ corresponding to 0 not being a pole of $\left.\zeta_{\mathcal{L}}\right)$, then it is a pole of $\zeta_{\partial \Omega, \Omega}$ of multiplicity $m+1$.

The identity (3.197) explains why the expressions for the fractal tube formulas in the case of simple poles to be discussed a little further on looked somewhat awkward in [Lap-vF4, §8.1] but are now significantly simplified, both conceptually and concretely. In particular, as is now clear, even in the present case when $N=$ 1 , the distance zeta function $\zeta_{\partial \Omega, \Omega}$ is the proper theoretical tool to define and understand the complex dimensions of fractal strings as well as to formulate the associated fractal tube formulas 110

In light of (3.196)-(3.197) and since $N=1$, the fractal tube formula (3.192) yields its counterpart for fractal strings (for every $\delta_{1} \geq \ell_{1} / 2$ ) 111

$$
\begin{aligned}
V_{\mathcal{L}}(\varepsilon) & =V_{\partial \Omega, \Omega}(\varepsilon) \\
& =\sum_{\omega \in \mathcal{D}_{\partial \Omega, \Omega}} \operatorname{res}\left(\frac{\varepsilon^{1-s}}{1-s} \zeta_{\partial \Omega, \Omega}(s), \omega\right)+R(\varepsilon) \\
& =\sum_{\omega \in \mathcal{D}_{\mathcal{L}} \cup\{0\}} \operatorname{res}\left(\frac{(2 \varepsilon)^{1-s}}{s(1-s)} \zeta_{\mathcal{L}}(s), \omega\right)+R(\varepsilon) \\
& =\sum_{\omega \in \mathcal{D}_{\mathcal{L}}} \operatorname{res}\left(\frac{(2 \varepsilon)^{1-s}}{s(1-s)} \zeta_{\mathcal{L}}(s), \omega\right)+\left\{2 \varepsilon \zeta_{\mathcal{L}}(0)\right\}_{0 \in U \backslash \mathcal{D}_{\mathcal{L}}}+R(\varepsilon),
\end{aligned}
$$

when $\mathcal{L}$ (i.e., $\zeta_{\mathcal{L}}$ ) is languid, and with $R(\varepsilon) \equiv 0$ when $\mathcal{L}$ (i.e., $\zeta_{\mathcal{L}}$ ) is strongly languid. Here, by definition, the term $\left\{2 \varepsilon \zeta_{\mathcal{L}}(0)\right\}_{0 \in U \backslash \mathcal{D}_{\mathcal{L}}}$ between braces in the last equality of (3.198) is included only if $0 \in U \backslash \mathcal{D}_{\mathcal{L}}$.

We note that the expression obtained in (3.198) is in complete agreement with the (pointwise or distributional) fractal tube formulas obtained for fractal strings in Lap-vF4, Thm. 8.1 or Thm. 8.7]) 112

${ }^{110}$ This is so even though the geometric zeta function has been (and will continue to be) a very useful tool as well. We note that an entirely analogous comment can be made about (not necessarily self-similar) fractal sprays; recall from 3.4 .10 that in the latter case, $\mathcal{L}$ may be unbounded and $\zeta_{\mathcal{L}}$ is then called the scaling zeta function of the fractal spray (see LapPe2, LapPeWi1 and Lap-vF4, §13.1]).

${ }^{111}$ Note that in light of 2.8) and (3.187), we have that $V_{\mathcal{L}}(\varepsilon)=V_{\partial \Omega, \Omega}(\varepsilon)$. Also, we let $\mathcal{D}_{\mathcal{L}}=\mathcal{D}_{\mathcal{L}}(U)$ and $\mathcal{D}_{\partial \Omega, \Omega}=\mathcal{D}_{\partial \Omega, \Omega}(U)$.

${ }^{112}$ In 2.1 for the simplicity of exposition, we gave a less precise statement of the formula; compare with formula 2.11). 
Since, in view of (3.196),

$$
\operatorname{res}\left(\zeta_{\partial \Omega, \Omega}, \omega\right)=\frac{2^{1-\omega}}{\omega} \operatorname{res}\left(\zeta_{\mathcal{L}}, \omega\right)
$$

for every simple complex dimension $\omega \in U \backslash\{0\}$, we deduce from (3.198) the following (pointwise or distributional) tube formula in the special case when all of the visible complex dimensions are simple (and $\zeta_{\mathcal{L}}$ is languid):

$$
\begin{aligned}
V_{\mathcal{L}}(\varepsilon) & =V_{\partial \Omega, \Omega}(\varepsilon) \\
& =\sum_{\omega \in \mathcal{D}_{\mathcal{L}}(W) \backslash\{0\}} \operatorname{res}\left(\zeta_{\mathcal{L}}, \omega\right) \frac{(2 \varepsilon)^{1-\omega}}{\omega(1-\omega)} \\
& +\left\{2 \varepsilon(1-\log (2 \varepsilon)) \operatorname{res}\left(\zeta_{\mathcal{L}}, 0\right)+2 \varepsilon \zeta_{\mathcal{L}}(0)\right\}_{0 \in U}+R(\varepsilon),
\end{aligned}
$$

where, by definition, the term between braces in the last equality of (3.200) is included only if $0 \in U$. Also, as before, if $\mathcal{L}$ (i.e., $\zeta_{\mathcal{L}}$ ) is strongly languid (which implies that $U=\mathbb{C}$ ), then we obtain an exact tube formula; that is, we can let $R(\varepsilon) \equiv 0$ in (3.200).

We point out that (3.200) is in agreement with the results stated in Lap-vF4. Cor. 8.3 or Cor. 8.10$]$ in the distributional or pointwise case, respectively

EXAMPLE 3.27. (The Sierpinski gasket). We briefly revisit the first example from $\$ 3.4$, studied in $\$ 3.4 .1$ in which $A \subseteq \mathbb{R}^{2}$ is the classic Sierpinski gasket and, by (3.68), all of the complex dimensions $s=0$ and $s_{k}:=\log _{2} 3+i(2 \pi / \log 2) k$ (for any $k \in \mathbb{Z}$ ) are simple with associated residues given by (3.69) and (3.70), respectively. Then, in light of (3.191) and since $N=2$ here, we obtain the following pointwise, exact fractal tube formula (the hypotheses of which are shown to be satisfied in LapRaZu1, Expl. 5.5.12]), valid for all $\varepsilon \in(0,1 / 2 \sqrt{3})$ :

$$
\begin{aligned}
V_{A}(\varepsilon) & =\sum_{k \in \mathbb{Z}} \operatorname{res}\left(\zeta_{A}, s_{k}\right) \frac{\varepsilon^{2-s_{k}}}{2-s_{k}}+\operatorname{res}\left(\zeta_{A}, 0\right) \frac{\varepsilon^{2-0}}{2} \\
& =\sum_{k \in \mathbb{Z}} \frac{6(\sqrt{3})^{1-s_{k}}}{4^{s_{k}}(\log 2) s_{k}\left(s_{k}-1\right)} \frac{\varepsilon^{2-s_{k}}}{2-s_{k}}+(3 \sqrt{3}+2 \pi) \frac{\varepsilon^{2}}{2} .
\end{aligned}
$$

Since $s_{k}=D+i k \mathbf{p}$, for all $k \in \mathbb{Z}$, where $D=\log _{2} 3$ and $\mathbf{p}=(2 \pi / \log 2)$ are, respectively, the Minkowski dimension and the oscillatory period of $A$, we can clearly rewrite (3.201) in the following form:

$$
V_{A}(\varepsilon)=\varepsilon^{2-D} G\left(\log _{2} \varepsilon^{-1}\right)+\left(\frac{3 \sqrt{3}+2 \pi}{2}\right) \varepsilon^{2},
$$

where $G$ is a continuous, nonconstant 1-periodic function, which is bounded away from zero and infinity, and is given by the following absolutely convergent (and hence, pointwise convergent) Fourier series expansion, for all $x \in \mathbb{R}$ :

$$
G(x):=\frac{6 \sqrt{3}}{\log 2} \sum_{k \in \mathbb{Z}} \frac{(4 \sqrt{3})^{-s_{k}}}{\left(2-s_{k}\right)\left(s_{k}-1\right) s_{k}} e^{i 2 \pi k x} .
$$

${ }^{113}$ Again, we note that in $\$ 2.1$ for the simplicity of exposition, the corresponding formula stated in 2.10) was not as precise as in 3.200. 
It is apparent from 3.203 that since $G$ is nonconstant, the function $\varepsilon^{-(2-D)} V_{A}(\varepsilon)$ is oscillatory, and hence does not have a limit as $\varepsilon \rightarrow 0^{+}$. Therefore, the Sierpinski gasket is not Minkowski measurable, in agreement with the Minkowski measurability criterion stated in $\$ 3.5 .2$ the hypotheses of which are easily verified since $D=\log _{2} 3$ is simple and $A$ has infinitely many nonreal principal complex dimensions (here, $s_{k}=D+i k \mathbf{p}$, with $k \in \mathbb{Z} \backslash\{0\}$ ).

EXAMPLE 3.28. (The Sierpinski carpet). Let $A \subseteq \mathbb{R}^{2}$ be the classic Sierpinski carpet studied in \$3.4.2. Then, in light of (3.71)-(3.73), (3.191) yields the following pointwise, exact fractal tube formula:

$$
V_{A}(\varepsilon)=\sum_{k \in \mathbb{Z}} \frac{2^{-s_{k}}}{(\log 3)\left(2-s_{k}\right)\left(s_{k}-1\right) s_{k}} \varepsilon^{2-s_{k}}+\frac{16}{5} \varepsilon^{2}+\frac{1}{2}\left(2 \pi+\frac{8}{7}\right) \varepsilon^{2},
$$

where $s_{k}:=D+i k \mathbf{p}$, for each $k \in \mathbb{Z}$; here, $D:=\log _{3} 8$ and $\mathbf{p}:=(2 \pi / \log 3)$ are, respectively, the Minkowski dimension and the oscillatory period of $A$.

ExERCISE 3.29. ( $i$ ) Much as in (3.202) and (3.203) above, rewrite the leading term (i.e., the sum over all $k \in \mathbb{Z}$ ) in (3.204) in the form $\varepsilon^{2-D} G\left(\log _{3} \varepsilon^{-1}\right)$, where $G$ is a continuous, nonconstant 1-periodic function, which is bounded away from zero and infinity.

(ii) Deduce from part $(i)$ via a direct computation that the Sierpinski carpet is not Minkowski measurable, also in agreement with the Minkowski measurability criterion stated in $\$ 3.5 .2$ (and of which you should verify the hypotheses).

(iii) Finally, by means of a direct computation (based, e.g., on part $(i)$ ), show that the Sierpinski carpet is Minkowski nondegenerate and calculate its average Minkowski content $\widetilde{\mathcal{M}}$. Furthermore, verify the latter results by using (3.51), connecting $\widetilde{\mathcal{M}}$ and the residue of $\zeta_{A}(s)$ at $s=D$ in the non-Minkowski measurable case (and of which the hypotheses are satisfied, in light of part $(i)$ ).

ExAmPLE 3.30. (The 3-d carpet). Let $A \subseteq \mathbb{R}^{2}$ be the 3 - $d$ carpet studied in 3.4.3. Then, in light of (3.76-3.80), (3.191) yields the following pointwise, exact fractal tube formula (valid for all $\varepsilon \in(0,1 / 2))$ :

$$
V_{A}(\varepsilon)=\frac{24}{13 \log 3} \varepsilon^{3-D} G\left(\log _{3} \varepsilon^{-1}\right)+\left(6-\frac{6}{17}\right) \varepsilon+\left(3 \pi+\frac{12}{23}\right) \varepsilon^{2}+\left(\frac{4 \pi}{3}-\frac{8}{25}\right) \varepsilon^{3},
$$

where $D=\log _{3} 26$ is the Minkowski dimension of $A$ and $G$ is a continuous, nonconstant 1-periodic function which is bounded away from zero and infinity, and is given by the following pointwise (absolutely convergent and hence) convergent Fourier series expansion:

$$
G(x)=\frac{24}{3 \log 3} \sum_{k \in \mathbb{Z}} \frac{2^{-s_{k}}}{\left(3-s_{k}\right)\left(s_{k}-1\right)\left(s_{k}-2\right) s_{k}} e^{i 2 \pi k x}, \text { for all } x \in \mathbb{R},
$$

with $\left(s_{k}:=D+i k \mathbf{p}\right)_{k \in \mathbb{Z}}$ denoting the sequence of principal complex dimensions of $A$ and $\mathbf{p}:=2 \pi / \log 3$ denoting the oscillatory period of $A$.

ExERCISE 3.31. (a) (3-d carpet, revisited). For the Sierpinski 3 - $d$ carpet $A$ in Example 3.30, answer the analog of questions $(i i)$ and $($ iii $)$ of Exercise 3.29.

(b) (3-gasket $R F D$ ). For the relative (or inhomogeneous) Sierpinski 3-gasket $\left(A_{3}, \Omega_{3}\right)$ studied in 3.4 .4 (specialized to $N=3$ ), use (3.191), along with the $N=3$ case of (3.82) and (3.89) (with $g_{3}=g_{3}(s)$ given by formula (3.94) of Exercise 3.9), in order to obtain a pointwise, exact fractal tube formula for $\left(A_{3}, \Omega_{3}\right)$. (Recall 
from the discussion following (3.92) that $D=\log _{2} 4=2$ is a complex dimension of $\left(A_{3}, \Omega_{3}\right)$ of multiplicity two, whereas the other complex dimensions are simple.)

Show via a direct computation that the $\operatorname{RFD}\left(A_{3}, \Omega_{3}\right)$ is Minkowski degenerate and therefore not Minkowski measurable but that with respect to the gauge function $h(t):=\log \left(t^{-1}\right)$, for all $t \in(0,1),\left(A_{3}, \Omega_{3}\right)$ is $h$-Minkowski measurable (and hence also $h$-Minkowski nondegenerate), as was stated towards the end of \$3.4.4 In order to establish the latter facts, you may use the results from LapRaZu6 and LapRaZu1, §5.4.4] briefly discussed towards the end of \$3.5.2.

EXAMPLE 3.32. (The $\frac{1}{2}$-square and $\frac{1}{3}$-square fractals). We revisit and complete here part $(a)$ (the $\frac{1}{2}$-square fractal) and part $(b)$ (the $\frac{1}{3}$-square fractal) of 33.4 .5

(a) (The $\frac{1}{2}$-square fractal). Recall from part $(a)$ of $\$ 3.4 .5$ that all of the fractal complex dimensions of the $\frac{1}{2}$-square fractal $A$ are simple, except for $s=1$, which is equal to $\operatorname{dim}_{B} A$. As a result, it follows from (3.191) in light of (3.98), (3.102) and (3.103) that the following pointwise, exact fractal tube formula holds (for all $\varepsilon \in(0,1 / 2))$ :

$$
V_{A}(\varepsilon)=\frac{1}{4 \log 2} \varepsilon \log \varepsilon^{-1}+\varepsilon G\left(\log _{2}(4 \varepsilon)^{-1}\right)+\frac{1+2 \pi}{2} \varepsilon^{2},
$$

where $G$ is a nonconstant, continuous 1-periodic function which is bounded away from zero and infinity and is given by the following convergent (because absolutely convergent) Fourier expansion:

$$
G(x):=\frac{29 \log 2-4}{8 \log 2}+\frac{1}{4} \sum_{k \in \mathbb{Z} \backslash\{0\}} \frac{e^{2 \pi i k x}}{\left(2-s_{k}\right)\left(s_{k}-1\right) s_{k}}, \text { for all } x \in \mathbb{R},
$$

with $s_{k}:=i k \mathbf{p}$, for all $k \in \mathbb{Z} \backslash\{0\}$, and $\mathbf{p}:=(2 \pi / \log 2)$, the oscillatory period of $A$.

Let us briefly explain how to obtain (3.207) and (3.208). In light of (3.192) (applied with $R(\varepsilon) \equiv 0$ because we are in the strongly $d$-languid case),

$$
\begin{aligned}
V_{A}(\varepsilon) & =\sum_{\omega \in \mathcal{D}\left(\zeta_{A}\right)} \operatorname{res}\left(\frac{\varepsilon^{2-s}}{2-s} \zeta_{A}(s), \omega\right) \\
& =\operatorname{res}\left(\frac{\varepsilon^{2-s}}{2-s} \zeta_{A}(s), 1\right)+\sum_{\omega \in \mathcal{D}\left(\zeta_{A}\right) \backslash\{1\}} \operatorname{res}\left(\zeta_{A}(s), \omega\right) \frac{\varepsilon^{2-\omega}}{2-\omega} .
\end{aligned}
$$

In order to calculate the above residue at $s=1$ in the last equality of (3.209), one computes the Laurent series expansion of $\zeta_{A}$ around $s=1$ (which is a double pole of $\zeta_{A}$ ), as follows:

$$
\zeta_{A}(s)=\frac{d_{-2}}{(s-1)^{2}}+\frac{d_{-1}}{s-1}+O(1) \quad \text { as } s \rightarrow 1,
$$

with

$$
d_{-2}:=\frac{1}{4 \log 2} \quad \text { and } \quad d_{-1}:=\frac{29 \log 2-2}{8 \log 2} .
$$

One then deduces from combining (3.210) and (3.211) that

$$
\begin{aligned}
\operatorname{res}\left(\frac{\varepsilon^{2-s}}{2-s} \zeta_{A}(s), 1\right) & =\varepsilon\left(d_{-1}-d_{-2}+d_{-2} \log \varepsilon^{-1}\right) \\
& =\frac{1}{4 \log 2} \varepsilon \log \varepsilon^{-1}+\frac{29 \log 2-4}{8 \log 2} .
\end{aligned}
$$


Finally, in light of (3.212) and the expression of $\operatorname{res}\left(\zeta_{A}(s), s_{k}\right)$ for any $k \in \mathbb{Z} \backslash\{0\}$ given in (3.103), as well as of the value of $\operatorname{res}\left(\zeta_{A}(s), 0\right)$ obtained in the just mentioned equation, we deduce the exact tube formula (3.207) from (3.209).

Next, recalling from (3.100) that $\operatorname{dim}_{B} A=1$, we can easily deduce from the fractal tube formula (3.207) that $A$ is Minkowski degenerate with Minkowski content $\mathcal{M}=+\infty$. In particular, $A$ is not Minkowski measurable, in the usual sense. Moreover, it follows from the $h$-Minkowski measurability criterion discussed at the end of 3.5.2, that for the choice of the gauge function $h(t):=\log t^{-1}$ (for all $t \in(0,1)), A$ is $h$-Minkowski measurable with $h$-Minkowski content $\mathcal{M}(A, h)$ given by

$$
\mathcal{M}(A, h)=\frac{1}{4 \log 2} .
$$

This concludes the discussion of the $\frac{1}{2}$-square fractal, for now.

(b) (The $\frac{1}{3}$-square fractal). Let us next briefly consider the $\frac{1}{3}$-square fractal $A \subseteq \mathbb{R}^{2}$ studied in part (b) of $\$ 3.4 .5$. Recall from that discussion (see, especially, (3.107), (3.109) and (3.112) that $\operatorname{dim}_{B} A=1, \operatorname{dim}_{P C} A=\{1\}$, and all of the complex dimensions of $A$ are simple with

$$
F \cup\{0,1\} \subseteq \mathcal{D}_{A} \subseteq\{0,1\} \cup\left\{s_{k}:=\log _{3} 2+i \mathbf{p} k: k \in \mathbb{Z}\right\},
$$

where $F$ is a nonempty finite subset of $\log _{3} 2+i \mathbf{p} \mathbb{Z}$ containing $\log _{3} 2$ as well as several nonreal complex dimensions (and conjectured to be infinite). Here and henceforth, $\mathbf{p}:=2 \pi / \log 3$.

Then, in light of the exact fractal tube formula (in the case of simple poles) stated in (3.191), combined with (3.214) and (3.109)-(3.112), we obtain the following pointwise, exact fractal tube formula for $A$ (valid for all $\varepsilon \in(0,1 / \sqrt{2})$ ):

$$
V_{A}(\varepsilon)=16 \varepsilon+\varepsilon^{2-\log _{3} 2} G\left(\log _{3}(3 \varepsilon)^{-1}\right)+\frac{12+\pi}{2} \varepsilon^{2},
$$

where $G$ is a nonconstant, continuous 1-periodic function which is bounded away from zero infinity and is given by the following absolutely convergent (and hence, pointwise convergent) Fourier series, for all $x \in \mathbb{R}$ :

$$
G(x):=\frac{1}{\log 3} \sum_{k \in \mathbb{Z}} \frac{e^{2 \pi i k x}}{\left(2-s_{k}\right) s_{k}}\left(\frac{6}{s_{k}-1}+\Psi\left(s_{k}\right)\right),
$$

where $\Psi=\Psi(s)$ is the entire function occurring in (3.105) and (3.111).

Finally, it follows from (3.215) that $A$ is Minkowski measurable with Minkowski content (in the usual sense) given by $\mathcal{M}=16$. This concludes for now our discussion of the $\frac{1}{3}$-square fractal.

EXERCISE 3.33. (a) (Cantor grill). Use the results of $\$ 3.4 .7$ to calculate the residues of $\zeta_{A}$ at the complex dimensions, and then to obtain a (pointwise, exact) fractal tube formula for the Cantor grill $A=C \times[0,1]$, where $C$ is the ternary Cantor set.

(b) (Cantor dust). Answer an analogous question for the Cantor dust $A=C \times C$ studied in 33.4.8. Then, extend your result to $A=C^{d}$, where $C^{d}$ is the Cartesian product of $d$ copies of the Cantor set $C$, with $d \geq 2$.

[Caution: Question (b) is more difficult than question (a).] 
Example 3.34. (The Cantor graph RFD). Let $(A, \Omega)$ denote the Cantor graph RFD (in $\mathbb{R}^{2}$ ) described and studied in $\$ 3.4 .9$. Recall that the compact set $A \subseteq \mathbb{R}^{2}$ is the graph of the Cantor function (also called the devil's staircase). Then, in light of (3.154)-(3.155), $D_{A, \Omega}=D_{A}=1$ and

$$
\mathcal{D}_{A, \Omega}=\{0,1\} \cup\left\{s_{k}:=\log _{3} 2+i k \mathbf{p}: k \in \mathbb{Z}\right\},
$$

with $\mathbf{p}:=2 \pi / \log 3$ and each complex dimension being simple. We therefore deduce from (3.191) the following pointwise, exact fractal tube formula, valid for every $\varepsilon \in(0,1)$ (where we use the values of the residues of $\zeta_{A}$ given in (3.156)-(3.157)):

$$
\begin{aligned}
V_{A, \Omega}(\varepsilon) & =2 \varepsilon+\varepsilon^{2-\log _{3} 2} G\left(\log _{3} \varepsilon^{-1}\right)+\varepsilon^{2} \\
& =2 \varepsilon^{2-D_{A, \Omega}}+\varepsilon^{2-D_{C S}} G\left(\log _{3} \varepsilon^{-1}\right),
\end{aligned}
$$

where (as above) $D_{A, \Omega}=D_{A}=1$, the dimension of the Cantor graph, and $D_{C S}=D_{C}=\log _{3} 2$, the dimension of the Cantor set (or of the Cantor string). Furthermore, in (3.218), $G$ is a nonconstant, continuous 1-periodic function which is bounded away from zero and infinity, and is given by the following absolutely convergent (and hence, pointwise convergent) Fourier series expansion, for all $x \in \mathbb{R}$ :

$$
G(x):=\frac{1}{\log 3} \sum_{k \in \mathbb{Z}} \frac{e^{2 \pi i k x}}{\left(2-s_{k}\right)\left(s_{k}-1\right) s_{k}} .
$$

Finally, it easily follows from (3.218)-(3.219) that $(A, \Omega)$ is Minkowski measurable, with Minkowski content given by

$$
\mathcal{M}_{A, \Omega}=\frac{\operatorname{res}\left(\zeta_{A, \Omega}, 1\right)}{2-1}=2,
$$

where we have used (3.157) in the second equality. Exactly the same property and identity as in (3.220) holds for the Cantor graph $A$ instead of for $(A, \Omega)$, as the interested reader can verify; in particular, $\mathcal{M}_{A}=2$. This concludes our discussion of the Cantor graph RFD for now. We will return to this example (and to the associated Cantor graph) in $\$ 3.6$, when discussing the notion of fractality; see the text surrounding (3.239) $-(3.245)$.

EXERCISE 3.35. Directly calculate the length of the Cantor graph (i.e., of the devil's staircase) $A$ and compare your result with the value of the Minkowski content of $A$ given above. Furthermore, give a heuristic, geometric argument that enables you to guess the length of $A$ without any computation.

EXAmPle 3.36. (Self-similar sprays). We briefly discuss the important class of self-similar sprays, studied in 33.4 .10 above. Since this discussion could be quite lengthy, otherwise, we refer to LapRaZu1, §5.5.6] for the details.

Let $(A, \Omega)$ be a self-similar spray with scaling ratios $r_{1}, \cdots, r_{J}$ (with $J \geq 2$ ) and (for simplicity, but without loss of generality) with a simple generator $G$ (or rather, generating or base $\operatorname{RFD}(\partial G, G))$, as in $\$ 3.4 .10$. Also as in $\$ 3.4 .10$, we assume that $G$ is a (nonempty) bounded open subset of $\mathbb{R}^{N}$, with $D_{\partial G, G}=\operatorname{dim}_{B}(\partial G, G)<N$, and that $\sum_{j=1}^{J} r_{j}^{N}<\infty$, so that the fractal spray has finite total volume. 
Recall that $\zeta_{A, \Omega}$ is then given by the key factorization formula 3.165) or (3.166), expressing $\zeta_{A, \Omega}$ in terms of the distance zeta function $\zeta_{\partial G, G}$ of the generating RFD and of the scaling zeta function $\zeta_{\mathfrak{s}}$ of the spray. Namely,

$$
\zeta_{A, \Omega}(s)=\zeta_{\mathfrak{s}}(s) \cdot \zeta_{\partial G, G}(s)=\frac{\zeta_{\partial G, G}(s)}{1-\sum_{j=1}^{J} r_{j}^{s}} .
$$

Furthermore, in light of (3.170), (3.173)-(3.174) and (3.176),

$$
D_{A, \Omega}=\max \left(\sigma_{0}, D_{\partial G, G}\right),
$$

where $\sigma_{0}=D\left(\zeta_{\mathfrak{s}}\right) \in(0, N)$ (the similarity dimension of the spray) is the unique real solution of the Moran equation Mora]; i.e., $\sigma_{0} \in \mathbb{R}$ and $\sum_{j=1}^{J} r_{j}^{\sigma_{0}}=1$. Moreover, in light of (3.173) - (3.174) and (3.176), if we assume that the generator $G$ is sufficiently "nice" [e.g., $G$ is monophase, in the sense of LapPe1-2, LapPeWi1-2 114 and, in particular, if $\partial G$ is a nontrivial polytope (by a result in [KoRati $)]$, we have that

$$
\mathcal{D}_{A, \Omega}=\mathcal{D}_{\partial G, G} \cup \mathcal{D}_{\mathfrak{s}} \subseteq\{0,1, \cdots, N-1\} \cup\left\{s \in \mathbb{C}: \sum_{j=1}^{J} r_{j}^{s}=1\right\},
$$

with frequent or "typical" equality in (3.223) and with $\mathcal{D}_{A, \Omega}$ always containing $D_{A, \Omega}$ and (by the results in Lap-vF4, Thm. 3]) also containing infinitely many (scaling) complex dimensions with real part $\sigma_{0}$.

We then deduce from (3.192) the following exact, pointwise fractal tube formula:

$$
V_{A, \Omega}(\varepsilon)=\sum_{\omega \in \mathcal{D}_{\mathfrak{s}} \cup\{0,1, \cdots, N-1\}} \operatorname{res}\left(\frac{\varepsilon^{N-s}\left(\sum_{\alpha=0}^{N} \kappa_{\alpha} \frac{g^{s-\alpha}}{s-\alpha}\right)}{(N-s)\left(1-\sum_{j=1}^{J} r_{j}^{s}\right)}, \omega\right) .
$$

Here, $g$ is the inner radius of the generator $G$ and the coefficients $\kappa_{\alpha}$ (some of which could vanish) are the coefficients of the polynomial expansion of $V_{\partial G, G}(\varepsilon) 115$

In the important special case when all of the scaling complex dimensions are simple and when $\sigma_{0}$ is not an integer, the fractal tube formula (3.224) takes the following simpler form:

$$
V_{A, \Omega}(\varepsilon)=\sum_{\omega \in \mathcal{D}_{\mathfrak{s}} \cup\{0,1, \cdots, N-1\}} d_{\omega} \varepsilon^{N-\omega},
$$

where

$$
d_{\omega}=\operatorname{res}\left(\zeta_{\mathfrak{s}}, \omega\right)\left(\sum_{\alpha=0}^{N} \frac{\kappa_{\alpha} g^{\omega-\alpha}}{\omega-\alpha}\right), \quad \text { if } \omega \in \mathcal{D}_{\mathfrak{s}},
$$

and

$$
d_{\omega}=\zeta_{\mathfrak{s}}(\omega) \kappa_{\omega}, \quad \text { if } \omega \in\{0,1, \cdots, N-1\}
$$

${ }^{114}$ Roughly, this means that $V_{\partial G, G}(\varepsilon)$ is polynomial for all $\varepsilon$ sufficiently small.

${ }^{115}$ More specifically,

$$
V_{\partial G, G}(\varepsilon)=\sum_{\alpha=0}^{N-1} \kappa_{\alpha} t^{N-\alpha}, \quad \text { for } 0<\varepsilon<g .
$$


Therefore,

$$
V_{A, \Omega}(\varepsilon)=\sum_{\omega \in \mathcal{D}_{\mathfrak{s}}} \operatorname{res}\left(\zeta_{\mathfrak{s}}, \omega\right)\left(\sum_{\alpha=0}^{N} \frac{\kappa_{\alpha} g^{\omega-\alpha}}{\omega-\alpha}\right)+\sum_{\alpha=0}^{N-1} \zeta_{\mathfrak{s}}(\alpha) \kappa_{\alpha} \varepsilon^{N-\alpha} .
$$

We note that if the generator $G$ is pluriphas 116 instead of monophase, then we can easily extend the above results and at the same time recover (as well as significantly extend) the results of [LapPe2 and, especially, of LapPeWi1]. Also, even if $G$ is not pluriphase (and thus certainly not monophase), it is clear from (3.221) that under suitable polynomial-type growth assumptions on $\zeta_{\partial G, G}$, one can use the tube formulas (from [LapRaZu6 and LapRaZu1, §§5.1-5.3]) recalled in 3.5.1 in order to obtain pointwise or distributional fractal tube formulas (with or without error term) for $(\partial G, G)$, expressed via $\zeta_{\partial G, G}$. This is so even if the fractal spray $\operatorname{RFD}(A, \Omega)$ is not necessarily self-similar. (Indeed, in that general case, the first equality in (3.221) still holds.) We let the interested reader elaborate on the latter comments.

As was already pointed out in $\$ 3.4 .10$ in the present case of self-similar sprays (and unlike for ordinary self-similar sets $A$ for which one always has $D_{A}=\sigma_{0}$ ), we must distinguish three cases here, all of which are realized (see, e.g., the example of the relative Sierpinski $N$-gasket discussed in 3.4 .4 and at the end of \$3.6.1) 117

Case $(i): D_{G}<\sigma_{0}$. Then, by (3.222), $D_{A, \Omega}=\sigma_{0}$ and is simple. Hence,

$$
\operatorname{dim}_{P C}(A, \Omega)=\mathcal{D}_{\mathfrak{s}}=\left\{s \in \mathbb{C}: \sum_{j=1}^{J} r_{j}^{s}=1\right\} .
$$

Consequently, in light of the structure of the scaling complex dimensions of selfsimilar sprays given in 3.4 .10 (and based on the results of Lap-vF4 Ch. 3]), we obtain the precise counterpart of the Minkowski measurablity criterion of selfsimilar strings from Lap-vF4 §8.4] (see part $(d)$ of Remark 2.4). Namely, the self-similar spray $\operatorname{RFD}(A, \Omega)$ is Minkowski measurable if and only if it is nonlattice; that is, if and only if it does not have any nonreal principal complex dimensions. Hence, if $(A, \Omega)$ is lattice, it is not Minkowski measurable, whereas if it is nonlattice, it is Minkowski measurable. This result follows from the refined version of the Minkowski measurability criterion stated in 3.5.2, and more precisely, from the necessary (respectively, sufficient) condition for Minkowski measurability obtained in LapRaZu1, §5.4] and in LapRaZu6 (and briefly alluded to in §3.5.2) 118

Case (ii): $D_{G}=\sigma_{0}$ (and hence, in light of (3.222), $D_{A, \Omega}=D_{G}=\sigma_{0}$ is an integer). Then, as was noted in $\$ 3.4 .10$, it follows from the factorization formula

\footnotetext{
${ }^{116}$ That is, roughly speaking, if $V_{\partial G, G}(\varepsilon)$ is a piecewise polynomial function; see LapPe2, LapPeWi1.

${ }^{117}$ Henceforth, we use the above notation and write $D_{G}:=D_{\partial G, G}=\operatorname{dim}_{B}(\partial G, G)$.

${ }^{118}$ Caution: The hypotheses of the Minkowski measurability criterion stated in 3.5 .2 are not always satisfied in the nonlattice case (see Lap-vF4 Exple. 5.32] for a counterexample). This is why we have to use a refined form (the aforementioned sufficient condition) in order to establish the Minkowski measurability of $(A, \Omega)$ in the nonlattice case. On the other hand, in the lattice case, the hypotheses of the above criterion are clearly satisfied and therefore we can conclude that $(A, \Omega)$ is not Minkowski measurable; alternatively, one can use the aforementioned sufficient condition (see LapRaZu1 Thm. 5.4.15]) in order to reach the same conclusion.
} 
(3.221) that $D_{A, \Omega}$ is a complex dimension of $(A, \Omega)$ of multiplicity two. As a result, due to the Minkowski measurability criterion discussed in $₫ 3.5 .2,(A, \Omega)$ cannot be Minkowski measurable (in the usual sense), irrespective of whether $(A, \Omega)$ is lattice or nonlattice. However, if we use the gauge function $h(t):=\log t^{-1}$, for all $t \in(0,1)$, then provided its hypotheses are satisfied, the $h$-Minkowski measurability criterion briefly discussed towards the end of $₫ 3.5 .2$ ([LapRaZu1, Thm. 5.4.32]), the RFD $(A, \Omega)$ is $h$-Minkowski measurable.

Case (iii): $D_{G}>\sigma_{0}$. Then, by (3.222), $D_{A, G}=D_{G}$. Since all of the scaling complex dimensions of $(A, \Omega)$ have real parts not exceeding $\sigma_{0}$ and hence, strictly less than $D_{A, \Omega}$, we deduce from (3.223) that $D_{A, \Omega}=D_{G}$ is the only principal complex dimension of $(A, \Omega)$ and (since $D_{G}$ is a simple pole of $\zeta_{\partial G, G}$ ) that it is simple. Consequently, in this case (i.e., in case $(i i i)),(A, \Omega)$ is always Minkowski measurable, whether or not the self-similar spray $(A, \Omega)$ is lattice or nonlattice.

However, this does not preclude $(A, \Omega)$ from having lower-order oscillations in its geometry (e.g., in its fractal tube formula (3.229) ). This is indeed what happens (generically) for the relative Sierpinski $N$-gasket when $N \geq 4$.

EXERCISE 3.37. (Relative $N$-gasket). By using the trichotomy outlined in cases (i)-( iii) just above, obtain (as explicitly as possible) fractal tube formulas for the relative (or inhomogeneous) Sierpinski $N$-gasket studied in 3.4.4. Also, determine the Minkowski measurability (or, more generally and when necessary, the $h$-Minkowski measurability) of the relative $N$-gasket, depending on the value of $N$. When appropriate, calculate the corresponding Minkowski content $\mathcal{M}$ or the average Minkowski content $\widetilde{\mathcal{M}}$.

[Hint: Distinguish the three cases when $N=2, N=3$ and $N \geq 4$, respectively.]

REMARK 3.38. (Ordinary self-similar sets.) (a) It has long been conjectured by the author (see Lap3. Conj. 3, p. 163]) that (classic or homogeneous) self-similar sets in $\mathbb{R}^{N}$ satisfying the open set condition (in the sense of Hut; see also Fa1] are Minkowski measurable if and only if they are nonlattice (and, equivalently, are not Minkowski measurable if and only if they are lattice). When $N=1$ (i.e., for selfsimilar strings), the fact that nonlattice self-similar sets (i.e., strings) are Minkowski measurable was first proved by the author in Lap3 and then, independently, by K. Falconer in [Fa2], in both cases by using the renewal theorem (first used in a related context by S. Lalley in Lall1-3] ). Then, this result was extended to higher dimensions (and to certain random fractals, as was also conjectured in Lap3) by D. Gatzouras in Gat. There remained to prove that the nonlattice condition was also necessary for obtaining the Minkowski measurability of a given self-similar set. This was first established when $N=1$ (i.e., for self-similar strings) by the author and M. van Frankenhuijsen in Lap-vF2 (see Lap-vF4, §8.4]), where both the necessary and sufficient conditions were proved by using the theory of complex dimensions of fractal strings (combined with a suitable Tauberian theorem) and the associated fractal tube formulas; see Lap-vF4, §8.4, Thms. 8.23 and 8.36]. Finally, in higher dimensions (i.e., when $N \geq 2$ ), the sufficient condition was recently established (independently of the above results from LapRaZu1 and the accompanying papers about self-similar sprays or RFDs) by S. Kombrink, E. Pearse and S. Winter in KomPeWi], also by using the renewal theorem but now combined with several nice new observations. 
(b) We conjecture that under suitable hypotheses and still for (classic or homogeneous) self-similar sets satisfying the open set condition, the above characterization expressed in terms of complex dimensions is still valid; that is, the presence of a nonreal principal complex dimension is equivalent to the self-similar set being not Minkowski measurable (and hence, Minkowski measurability is equivalent to the Minkowski dimension $D$ being the only principal complex dimension; see LapRaZu1, $\mathrm{Pb}$. 6.2.36] for more details. This problem still remains open, for now, and its resolution will require, in particular, suitably extending the factorization formula (3.221) to this setting or finding an appropriate substitute for it.

3.6. Fractality, hyperfractality and unreality, revisited. We pursue and complete here the discussion of fractality and unreality (as well as of the closely related topic of the meaning of complex dimensions) started in \$2.4 and \$2.5. In light of the higher-dimensional theory of complex dimensions developed in LapRaZu1 (and in the accompanying series of papers, LapRaZu2-10) and, in particular, of the fractal tube formulas obtained in LapRaZu1, Ch. 5] and LapRaZu6 (as well as discussed in 3.5 .1 and 3.5 .3 ), the interpretation of (necessarily complex conjugate pairs of) nonreal complex dimensions as giving rise to (or detecting) the intrinsic oscillations of a given geometric object is exactly the same as in the one-dimensional case of fractal strings discussed in $\$ 2.4$. Therefore, we refer the interested reader to \$2.4 for the corresponding discussion, which can easily be adapted to higher dimensions and is illustrated by the many examples of complex dimensions and fractal tube formulas provided in $\$ 3.4$ and 3.5 .3 respectively.

We further mention that for clarity, we will focus here on geometric objects which are relative fractal drums or RFDs (and, in particular, bounded sets) in $\mathbb{R}^{N}$. However, since the entire theory of fractal zeta functions and complex dimensions (in particular, of the associated fractal tube formulas) extends (essentially without change) to suitable metric measure spaces (namely, Ahlfors-type spaces and beyond), as is shown in [LapWat, Wat, we could instead work within the much greater generality of RFDs (and, in particular, of bounded sets) in such metric measure spaces. (See Remark 3.25]) The main difference is that the embedding dimension $N$ would have to be replaced by the Ahlfors dimension (or its appropriate analog) of the embedding metric measure space; see footnote 109

Perhaps more importantly, we could also apply to various counting functions the very general (pointwise or distributional) explicit formulas from Lap-vF4, Ch. $5]$ in order to detect the potential geometric, spectral, dynamical, or arithmetic oscillations that are intrinsic to fractal-like (physical or mathematical) objects. These counting functions could be geometric (such as the box-counting function), spectral (such as the eigenvalue or frequency counting function or else, the partition function or trace of the heat semigroup) or dynamical (such as the prime orbit counting function, counting the number of homology classes of primitive periodic orbits of the corresponding dynamical system 119 ).

Moreover, in a similar spirit, we could use other types of fractal-like zeta functions (such as, for example, suitably weighted Ruelle's dynamical zeta functions;

${ }^{119}$ See [Lap-vF4 Ch. 7] for a simple but illuminating example. The author has long thought that the just referred work could be greatly extended to a variety of hyperbolic and other dynamical systems, for example, within the setting of the theory developed by Parry and Pollicott in ParrPol1, ParrPol2, where Ruelle or dynamical zeta functions (Rue1-4, Lag) were used. This potentially significant extension still remains to be achieved. 
see, e.g., Rue1-4, ParrPol1-2, Lag, along with footnote 119) could be used to define "fractality" via the existence of nonreal complex dimensions. Finally, as will be further explained below, even the notion of "complex dimensions" itself can be relaxed, by considering (nonremovable) singularities that are not just poles of the associated fractal zeta functions.

Accordingly, the proposed notion of fractality can be potentially applied to a great variety of settings in mathematics, physics, cosmology, chemistry, biology, medicine, geology, computer science, engineering, economics, finance, and the arts, as well as can be illustrated via many different kinds of fractal-type explicit formulas. In mathematics or physics alone, the corresponding fields involved would include, for instance, harmonic analysis, partial differential equations, geometric measure theory, spectral theory, spectral geometry, probability theory, dynamical systems, combinatorics and graph theory, number theory and arithmetic geometry, algebraic geometry, operator algebras and noncommutative geometry, quantum groups, mathematical physics, along with, on the more physical side, condensed matter physics, astronomy and cosmology, quantum theory and its myriad of applications, quantum gravity, classical and quantum chaos, quantum computing and string theory. For the simplicity of exposition, however, and with one single exception 120 we will limit our discussion to the geometric setting, that of RFDs (and in particular, of bounded sets) in an $N$-dimensional Euclidean space $\mathbb{R}^{N}$, as well as briefly illustrate it by means of the fractal tube formulas and the Minkowski measurability results described in $\S \$ 3.5 .13 .5 .3$

As in 2.5. we say that a geometric object (e.g., a fractal drum $(A, \Omega)$ in $\mathbb{R}^{N}$ or, in particular, a bounded subset $A$ of $\mathbb{R}^{N}$ ) is fractal if it has at least one nonreal complex dimension (and hence, at least one pair of nonreal complex conjugate complex dimensions). For now, "complex dimensions" are interpreted as being the (visible) poles of the associated fractal zeta function $\zeta_{A, \Omega}$ or, equivalently, $\widetilde{\zeta}_{A, \Omega}$ of $(A, \Omega) 121$ However, we will further broaden this notion later on in this subsection; see, especially, 33.6 .2 and 33.6 .3 This definition is formally identical to the one proposed in Lap-vF4, Ch. 12] (and, prior to that, in Lap-vF1-3) 122 but it is worth pointing out that at the time, there was no suitable general definition of fractal zeta functions, and hence also of complex dimensions, that was compatible with the existence of fractal tube formulas for compact subsets of $\mathbb{R}^{N}$, with $N \geq 2$.

More specifically, given $d \in \mathbb{R}$, an $\operatorname{RFD}(A, \Omega)$ (and, in particular, a bounded set $A$ ) in $\mathbb{R}^{N}$ is said to be fractal in dimension $d$ if it has at least one (and hence,

${ }^{120}$ This exception will have to do with the spectra of fractal drums; see 3.6 .4

${ }^{121}$ For simplicity, we assume from now on that $D:=\overline{\operatorname{dim}_{B}}(A, \Omega)<N$, so that the distance and tube zeta functions $\zeta_{A, \Omega}$ and $\widetilde{\zeta}_{A, \Omega}$ have the same visible poles in a given domain $U$ of $\mathbb{C}$ to which one (and hence, both) of these fractal zeta function has a (necessarily unique) meromorphic extension; in particular, $D\left(\zeta_{A, \Omega}\right)=D\left(\widetilde{\zeta}_{A, \Omega}\right)=D$. (See 3.3 .1 and 3.3 .2 ) If one wants to deal with the case when $D=N$, one should then work with $\widetilde{\zeta}_{A, \Omega}$ alone.

${ }^{122}$ There is one small difference; namely, we no longer require the real part of the nonreal complex dimension to be positive (a condition that was included mainly for aesthetic reasons and is fulfilled, for example, by classic self-similar geometries). Indeed, for an $\operatorname{RFD}(A, \Omega)$, even $D=\overline{\operatorname{dim}_{B}}(A, \Omega)$ can be negative (or equal to $-\infty$ ); see the two examples provided between (3.4) and (3.7) in 3.2 
a pair of complex conjugate) complex dimension(s) of real part $d 123$ Therefore, by definition, fractality is equivalent to fractality in some dimension $d \in \mathbb{R}$.

Moreover, $(A, \Omega)$ (or $A$ ) is said to be critically fractal if it is fractal in dimension $D$, the (upper) Minkowski dimension of $(A, \Omega)$ (or of $A$ ); in other words, if and only if it has at least one nonreal complex dimension. Otherwise $(A, \Omega)$ (or $A$ ) is said to be subcritically fractal; in that case, it is therefore fractal in some dimension $d<D$ but not in dimension $D$.

In addition, the $\operatorname{RFD}(A, \Omega)$ is said to be hyperfractal if the associated fractal zeta function $\zeta_{A, \Omega}$ (or equivalently, $\widetilde{\zeta}_{A, \Omega}$, since $D<N$ here) cannot be meromorphically extended to a connected open neighborhood of a suitable curve or contour $S$ in $\mathbb{C}$ extending in both vertical directions (i.e., $S$ is a screen, in the sense of Lap-vF4 or LapRaZu1), and it is said to be critically hyperfractal (or strongly hyperfractal, as in LapRaZu1 ) if $S=\{\operatorname{Re}(s)=D\}$, and maximally hyperfractal if the critical line $\{\operatorname{Re}(s)=D\}$ consists solely of (nonisolated and nonremovable) singularities of $\zeta_{A, \Omega}$ (or equivalently, of $\widetilde{\zeta}_{A, \Omega}$ ) 124 (Naturally, a similar terminology is used in the special case of a bounded subset $A$ of $\mathbb{R}^{N}$.)

Clearly, "maximally hyperfractal" implies "critically hyperfractal", which itself implies "hyperfractal". Furthermore, maximal hyperfractals are such that $\zeta_{A, \Omega}$ (or equivalently, $\widetilde{\zeta}_{A, \Omega}$ ) have nonreal (complex conjugate pairs of) singularities (which are not necessarily poles). Moreover, in some sense, they are among the most complicated fractals.

In LapRaZu1, \$4.6] (and LapRaZu3) are constructed maximally hyperfractal compact sets as well as RFDs in $\mathbb{R}^{N}$, for $N \geq 1$ arbitrary (and, in particular, when $N=1$, maximally hyperfractal fractal strings), with any prescribed (upper) Minkowski dimension $D \in(0, N)$. In fact, the family of examples constructed in loc. cit. consists not only of maximally hyperfractal but also of transcendentally $\infty$-quasiperiodic sets or RFDs. Recall from LapRaZu1, §4.6] that an RFD $(A, \Omega)$ (or a bounded set $A$ ) in $\mathbb{R}^{N}$, of (upper) Minkowski dimension $D$ is said to be transcendentally $\infty$-quasiperiodic if its tube function $V=V(\varepsilon)$ (where $V:=V_{A, \Omega}$ or $\left.V:=V_{A}\right)$ satisfies

$$
V(\varepsilon)=\varepsilon^{N-D}\left(G\left(\log \varepsilon^{-1}\right)+o(1)\right) \quad \text { as } \varepsilon \rightarrow 0^{+},
$$

where the function $G: \mathbb{R} \rightarrow \mathbb{R}$ is transcendentally $\infty$-quasiperiodic; i.e., where $G=$ $G(t)$ is the restriction to the diagonal of a nonconstant function $H: \mathbb{R}^{\infty} \rightarrow \mathbb{R}$ which is separately periodic (of minimal period $T_{j}$ ) in each variable $t_{j}$, for $j=1,2, \cdots$ :

$$
G(t)=H(t, t, t, \cdots), \quad \text { for all } t \in \mathbb{R}
$$

where (for all $\left.\left(t_{1}, t_{2}, \cdots\right) \in \mathbb{R}^{\infty}\right)$

$$
H\left(t_{1}, t_{2}, \cdots, t_{j-1}, t_{j}+T_{j}, t_{j+1}, \cdots\right)=H\left(t_{1}, t_{2}, \cdots, t_{j-1}, t_{j}, t_{j+1}, \cdots\right) .
$$

\footnotetext{
${ }^{123}$ Then, clearly, we must have $D>-\infty$ and $d \leq D \leq N$, where $D:=\overline{\operatorname{dim}}_{B}(A, \Omega)$ or $\left(D:=\overline{\operatorname{dim}}_{B} A\right)$. Also, note that $d$ itself need not be a (nonremovable) singularity, let alone a pole, of $\zeta_{A, \Omega}\left(\right.$ or of $\left.\widetilde{\zeta}_{A, \Omega}\right)$.

${ }_{124}$ Precise definitions of the notion of a singularity (of a complex-valued function on a domain of $\mathbb{C})$ can be found in [LapRaZu1, §1.3.2] and the references therein; see also [LapRaZu1 §4.6.3].
} 
In addition, the use of the adverb "transcendentally" in the above definition means that the resulting sequence of quasiperiods $\left(T_{j}\right)_{j \geq 1}$ is linearly independent over the field of algebraic numbers 125

The aforementioned construction of maximally hyperfractal and transcendentally $\infty$-quasiperiodic RFDs is rather complicated. Its first (and main) step consists in constructing fractal strings with the above properties. In essence, these highly complex fractal strings are obtained by taking the countable disjoint union of a suitable sequence extracted from a two-parameter family of topological Cantor sets (or strings), and then to appropriately apply a key result from transcendental number theory (namely, Baker's theorem [Bak]; see also LapRaZu1, Thm. 3.114]) 126 In some sense, this construction can be viewed as a fractal-geometric interpretation of Baker's theorem.

We close this discussion by providing several examples of critical and subcritical fractals.

First, we note that under suitable hypotheses (namely, the hypotheses of the Minkowski measurability criterion stated in $\$ 3.5 .2$ ), and supposing that the Minkowski dimension $D=\operatorname{dim}_{B}(A, \Omega)$ exists and is simple 127 the $\operatorname{RFD}(A, \Omega)$ in $\mathbb{R}^{N}$ is subcritically fractal if and only if it is Minkowski measurable. Stated another way, and still under the hypotheses of the aforementioned criterion, $(A, \Omega)$ is critically fractal if and only if it is not Minkowski measurable. Naturally, the same statements hold for bounded subsets $A$ of $\mathbb{R}^{N}$.

Moreover, self-similar strings are subcritically fractal if and only if they are nonlattice, and also if and only if they are Minkowski measurable. (See Lap-vF4, $\S 8.4]$ and part $(d)$ of Remark 2.4, 128 Equivalently, self-similar strings are critically fractal if and only if they are lattice, and also if and only if they are not Minkowski measurable (even though they are always Minkowski nondegenerate).

As was mentioned in Example 3.36, exactly the same statements (as just above for self-similar strings) hold for self-similar fractal spray RFDs (in $\mathbb{R}^{N}, N \geq 1$ ) with "nice" generators (e.g., monophase or even pluriphase generators, and in particular, with generators that are nontrivial polytopes), in case (i) of Example 3.36 i.e., when

\footnotetext{
${ }^{125}$ Recall that the field of algebraic numbers can be viewed (up to isomorphism) as the algebraic closure $\overline{\mathbb{Q}}$ of $\mathbb{Q}$, the field of rational numbers. It is obtained by adjoining to $\mathbb{Q}$ the complex roots of all of the monic polynomials with coefficients in $\mathbb{Q}$ (or equivalently, in $\mathbb{Z}$ ). By reasoning by absurdum, one can easily check that it is a countable set.

${ }^{126}$ Recall that Baker's theorem states that given $n \in \mathbb{N}$ with $n \geq 2$, if $m_{1}, \cdots, m_{n}$ are positive algebraic numbers such that $\log m_{1}, \cdots, \log m_{n}$ are linearly independent over the rationals, then $1, \log m_{1}, \cdots, \log m_{n}$ are linearly independent over the field of algebraic numbers (i.e., algebraically independent). In particular, $\log m_{1}, \cdots, \log m_{n}$ are transcendental (i.e., not algebraic) numbers, and so are their pairwise quotients.

${ }^{127}$ If $D$ is multiple (as a pole of $\zeta_{A, \Omega}$ ), then it follows from LapRaZu1 Thm. 5.4.27] briefly discussed towards the end of 33.5 .2 that under the hypotheses of that theorem, the $\operatorname{RFD}(A, \Omega)$ is $h$-Minkowski measurable with respect to the gauge function $h(t):=\left(\log t^{-1}\right)^{m-1}$, for all $t \in(0,1)$, where $m \geq 2$ is the multiplicity of $D$.

${ }^{128}$ We caution the reader that this statement is not a direct consequence of the general Minkowski measurability criterion (in terms of nonreal principal complex dimensions) used above and stated in 3.5 .2 Indeed, the hypotheses of the corresponding theorem are not satisfied by all nonlattice self-similar strings. However, as was briefly mentioned in Example 3.36 of LapRaZu1. $\S 5.4]$, the extension of this statement to self-similar sprays with nice generators is proved by using separately the necessary condition and the sufficient condition for Minkowski measurability obtained in loc. cit.
} 
$D_{G}:=\operatorname{dim}_{B}(\partial G, G)<\sigma_{0}$, the similarity dimension of $(A, \Omega)$, where $(\partial G, G)$ is the generator (or base) of the self-similar spray $\operatorname{RFD}(A, \Omega)$.

In addition, irrespective of whether we are in case $(i),(i i)$ or $(i i i)$ of Example 3.36, it follows from the results of Lap-vF4, Ch. 3] combined with (3.223) and the text following it, that self-similar sprays (with nice generators) are always fractal, and more specifically, that lattice (respectively, nonlattice) sprays are fractal in dimension $d$ for finitely (respectively, countably infinitely) many values of $d \in \mathbb{R}$. Also, such lattice sprays are critically fractal whereas nonlattice sprays are subcritically fractal. Furthermore, still in light of those results, but now also combined with the main theorem in MorSepVi1] (proving and extending a conjecture in Lap-vF5, see also Lap-vF3), it is known that the set of $d$ 's for which a given nonlattice spray is fractal in dimension $d$ is dense in a single compact (nonempty) interval $\left[D_{\ell}, D\right]$ in the generic case, with $D_{\ell}<D$, while we conjecture that an analogous statement is true in the nongeneric case, but now with finitely many (but more than one) compact nonempty intervals instead of a single one 129

3.6.1. The $\frac{1}{2}$-square fractal, the $\frac{1}{3}$-square fractal and the relative Sierpinski $N$-gasket. The above results can be illustrated by the $\frac{1}{2}$-square fractal RFD, the $\frac{1}{3}$-square fractal RFD and (for suitable values of $N$ ) the relative $N$-gasket.

(a) More specifically, for the $\frac{1}{2}$-square fractal RFD $(A, \Omega), D:=\operatorname{dim}_{B}(A, \Omega)=1$ is a complex dimension of multiplicity two; hence, $(A, \Omega)$ is not Minkowski measurable but is also Minkowski degenerate, with Minkowski content $\mathcal{M}=+\infty$. However, $(A, \Omega)$ is $h$-Minkowski measurable with respect to the gauge function $h(t)=$ $\log t^{-1}$ (for all $\left.t \in(0,1)\right)$ and with $h$-Minkowski content $\mathcal{M}((A, \Omega), h)=1 / 4 \log 2$. (See part $(a)$ of 33.4 .5 and part $(a)$ of Example 3.32.) Further, by (3.100)-(3.102),

$$
\begin{gathered}
D:=D_{A, \Omega}=1, \\
\mathcal{D}_{A, \Omega}=\{0,1\} \cup\left(1+i \frac{2 \pi}{\log 2} \mathbb{Z}\right)
\end{gathered}
$$

and

$$
\operatorname{dim}_{P C}(A, \Omega)=1+i \frac{2 \pi}{\log 2} \mathbb{Z} .
$$

Therefore, the $\frac{1}{2}$-square fractal $(A, \Omega)$ is critically fractal, and is only fractal in dimension $D=1$, the Minkowski dimension of $(A, \Omega)$. According to loc. cit., the same statements are true for the $\frac{1}{2}$-square fractal $A$ (instead of for $(A, \Omega)$ ).

(b) Next, let $(A, \Omega)$ be the $\frac{1}{3}$-square fractal, as in part $(b)$ of 3.4 .5 and in part (b) of Example 3.32, Then, in light of (3.109) and the discussion surrounding it, $(A, \Omega)$ is subcritically fractal in dimension $d:=\log _{3} 2$ and is only fractal in that dimension, the Minkowski dimension of the ternary Cantor set. Furthermore, $D:=D_{A, \Omega}=1$ and 1 is simple (as well as the only principal complex dimension

${ }^{129}$ We conjecture that under suitable hypotheses, analogous results hold for the scaling complex dimension of (classic or homogeneous) self-similar sets satisfying the open set condition (as in Hut and, e.g., Fa1 ); in particular, all such self-similar sets are fractal. A key open problem in this context is to first obtain a factorization formula of the type (3.221) (see also (3.165)), possibly up to the addition of a holomorphic function in an appropriate right half-plane $\{\operatorname{Re}(s)>\beta\}$, for some $\beta<D$. (See also LapRaZu1 Pb. 6.2.36 and Rem. 6.2.37].) 
of $(A, \Omega))$. Moreover, according to part (b) of Example 3.32, the $\frac{1}{3}$-square fractal $\operatorname{RFD}(A, \Omega)$ is Minkowski measurable. In light of loc. cit., the exact same results hold for the $\frac{1}{3}$-square fractal $A$ itself.

(c) We now consider the relative $N$-gasket $\left(A_{N}, \Omega_{N}\right)$, studied for any $N \geq 2$ in 33.4.4. Recall that with the notation used above for self-similar sprays, $D_{G}=N-1$ and $\sigma_{0}=\log _{2}(N+1)$, and in light of (3.89) and (3.91), that

$$
\mathcal{D}_{A_{N}, \Omega_{N}}=\{0,1, \cdots, N-1\} \cup\left(\log _{2}(N+1)+i \frac{2 \pi}{\log 2} \mathbb{Z}\right)
$$

and

$$
D:=\operatorname{dim}_{B}\left(A_{N}, \Omega_{N}\right)=\max \left(N-1, \log _{2}(N+1)\right)= \begin{cases}\log _{2} 3, & \text { if } N=2, \\ N-1, & \text { if } N \geq 3\end{cases}
$$

Therefore, as we next explain, we recover the three cases $(i),(i i)$ and (iii) discussed above for general self-similar spray RFDs:

Case $(i)$ when $D_{G}<\sigma_{0}$ corresponds to the $N=2$ case; then, $\left(A_{2}, \Omega_{2}\right)$ is not Minkowski measurable but is Minkowski nondegenerate. Also, it is critically fractal and fractal only in dimension $d=\log _{2} 3$, the dimension of the relative Sierpinski gasket $\left(A_{2}, \Omega_{2}\right)$. In light of the results of 3.2 .1 exactly the same statements hold for the (classic) Sierpinski gasket $A_{2}$ itself.

Case (ii) when $D_{G}=\sigma_{0}$ (i.e., $N-1=\log _{2}(N+1)$ ) corresponds to $N=3$. Then, $\left(A_{3}, \Omega_{3}\right)$ is not Minkowski measurable (because $D=2$ is of multiplicity two) and is also Minkowski degenerate. However, it is $h$-Minkowski measurable with respect to the gauge function $h(t):=\log t^{-1}$ (for all $t \in(0,1)$ ). Furthermore, in light of (3.237) and (3.238), $\left(A_{3}, \Omega_{3}\right)$ is critically fractal (necessarily in dimension $\left.d:=D=\log _{2} 4=2\right)$, and is fractal only in that dimension.

Finally, case (iii) when $D_{G}>\sigma_{0}$ corresponds to every value of $N \geq 4$. In this case, $D=D_{G}=N-1,\left(A_{N}, \Omega_{N}\right)$ is subcritically fractal in dimension $d:=\sigma_{0}=\log _{2}(N+1)$, and is fractal only in that dimension. Also, it is Minkowski measurable but has geometric oscillations of lower order (corresponding to the nonreal complex dimensions of real part $\log _{2}(N+1)$ ). This concludes our discussion of the relative Sierpinski $N$-gasket.

3.6.2. The devil's staircase and fractality. We close this part of the discussion by considering the emblematic example of the Cantor graph RFD $(A, \Omega)$ studied in 3.4.9 and in Example 3.34. This example is closely related to the Cantor graph $A$, also called the "devil's staircase" in $\mathbf{M a n}$. It is important for a variety of reasons:

(a) First, $(A, \Omega)$ is not a self-similar spray RFD. Indeed, the devil's staircase $A$ is not a self-similar set; instead, it is a self-affine set, which makes the corresponding computation significantly more complicated, if not impossible to carry out.

(b) Secondly, the devil's staircase is not fractal according to Mandelbrot's definition of fractality (to be recalled just below), even though everyone with an exercised 
eye (including Benoit Mandelbrot himself) 130 would agree that it must be "fractal" 131 At this point, it may be helpful to the reader to recall that in Man (and in later work), Mandelbrot called "fractal" any (bounded) subset $A$ of Euclidean space $\mathbb{R}^{N}$ such that its topological dimension $\operatorname{dim}_{T} A$ and Hausdorff dimension $\operatorname{dim}_{H} A$ do not coincide 132

$$
\operatorname{dim}_{H} A \neq \operatorname{dim}_{T} A,
$$

or, equivalently, since it is always true that $\operatorname{dim}_{T} A \leq \operatorname{dim}_{H} A$,

$$
\operatorname{dim}_{H} A>\operatorname{dim}_{T} A .
$$

In the present case when $A$ is the devil's staircase, this definition clearly fails (as Mandelbrot was well aware of). Indeed, since the Cantor graph is a rectifiable curve (i.e., a curve of finite length), we have that

$$
\operatorname{dim}_{H} A=\operatorname{dim}_{B} A=\operatorname{dim}_{T} A=1,
$$

according to a well-known result in elementary geometric measure theory (see [Fed1]) and as can also be directly checked here.

(c) Lastly, we mention that the unambiguous and frustrating contradiction between the visual impression one gets when contemplating the devil's staircase and Mandelbrot's definition of fractality (which he only adopted reluctantly, in response to the many queries and criticisms he received after the publication of his earlier French book on fractals) led the present author to wonder how to resolve this paradox and more importantly, how to much better capture the intuition underlying the informal notion of "fractality". Combined with the author's early work on fractal drums Lap1-4 and his joint work on fractal strings and the Riemann zeta function as well as the Riemann hypothesis LapPo1-3, LapMa1-3, this quest eventually led (first, for fractal strings, in Lap-vF1-4 and then, in any dimension $N \geq 1$, in LapRaZu1) to the notion of complex fractal dimensions and to the present notion of fractality expressed in terms of the irreality (or the "unreality") of some of the underlying complex dimensions.

Now, let us return to the Cantor graph $\operatorname{RFD}(A, \Omega)$ and its complex dimensions. In light of the discussion of that example provided in 3.4 .9 and in Example 3.34.

$$
\begin{gathered}
D:=D_{A, \Omega}=D_{A}=1, \\
\{1\} \subseteq \mathcal{D}_{A} \subseteq \mathcal{D}_{A, \Omega}=\{0,1\} \cup\left(\log _{3} 2+i \frac{2 \pi}{\log 3} \mathbb{Z}\right)
\end{gathered}
$$

\footnotetext{
${ }^{130}$ See the very explicit and enlightening comments in Man p. 82] and Man Plate 83, p. 83]; see also the very convincing and beautiful figure in loc. cit. Let us quote from [Man p. 82]: "One would love to call the present curve a fractal, but to achieve this goal, we would have to define fractal less stringently on the basis of notions other than $D$ [the Hausdorff dimension] alone."

${ }^{131}$ The exact same comment can be made verbatim about the Cantor graph RFD provided, in Mandelbrot's original definition, one substitutes the (relative) Minkowski dimension for the Hausdorff dimension.

${ }^{132}$ It is noteworthy that if $A$ is nonempty, $\operatorname{dim}_{T} A$ is always a nonnegative integer. For example, for the ternary Cantor set, it is equal to 0 while for the classic Sierpinski gasket and for the Koch snowflake curve, it is equal to 1.
} 
and hence,

$$
\operatorname{dim}_{P C} A=\operatorname{dim}_{P C}(A, \Omega)=\{1\},
$$

where (in (3.243) ) $D_{C S}=\log _{3} 2$ is the Minkowski dimension of the ternary Cantor set. In addition, it is conjectured (first in Lap-vF2-4, via an approximate computation of the corresponding tube function, and then, with significantly more precise supporting arguments, in LapRaZu1,4) that

$$
\log _{3} 2+i \frac{2 \pi}{\log 3} \mathbb{Z} \subseteq \mathcal{D}_{A}
$$

or even that $\mathcal{D}_{A}=\mathcal{D}_{A, \Omega}$ in (3.243).

The Cantor graph RFD is Minkowski measurable; furthermore, it is fractal in dimension $d:=D_{C S}=\log _{3} 2$ and is fractal only in that dimension. Consequently, it is critically subfractal. The presence of the nonreal complex (and subcritical) dimensions $s_{k}=\log _{3} 2+i(2 \pi / \log 3) k$ (with $\left.k \in \mathbb{Z} \backslash\{0\}\right)$ gives rise to logarithmically (or multiplicatively) periodic oscillations of order $D_{C S}=\log _{3} 2<1133$ (See the fractal tube formula (3.218) along with (3.219).) Therefore, it has a real effect on the geometry. According to the aforementioned conjecture (see (3.245) and the text following it), the devil's staircase (or Cantor graph) $A$ itself should have exactly the same qualitative and quantitative properties as the Cantor graph RFD $(A, \Omega)$. In particular, it is not critically fractal but is critically fractal in dimension $d:=D_{C S}=\log _{3} 2$.

The following open problem is technically very challenging but also conceptually important, in light of the above discussion.

Open Problem 3.39. (Complex dimensions of the devil's staircase.) Prove the conjecture stated in (3.245) or even its stronger form stated just afterward.

As a first but important step towards that conjecture, try to show that there are at most finitely many exceptions to the inclusion appearing in (3.245) or at least, that infinitely many elements of the left-hand side of the inclusion in (3.245) belong to $\mathcal{D}_{A}$.

3.6.3. Extended notion of complex dimensions, scaling laws and Riemann surfaces. We continue this discussion by broadening the notion of complex dimensions and correspondingly, of fractality. As was alluded to earlier, it is natural to call "complex dimensions" of an RFD $(A, \Omega)$ not only the poles but also other types of (nonremovable) singularities of the associated fractal zeta function $\zeta_{A, \Omega}$ (or, equivalently, $\widetilde{\zeta}_{A, \Omega}$, provided $\left.D:=\operatorname{dim}_{B}(A, \Omega)<N\right)$. Typically, the (necessarily closed) set of singularities is obtained as the closure of a countable (or finite) set of "geometric singularities", which we propose to call the kernel or simply, the set of "geometric singularities" of $(A, \Omega) 134$ For example, for each member $(A, \Omega)$, say, of the family of (transcendentally $\infty$-quasiperiodic) maximally hyperfractal RFDs or compact sets constructed in LapRaZu1,3 and discussed earlier, the kernel consists

\footnotetext{
${ }^{133}$ More precisely, they correspond to the term $\varepsilon^{2-D_{C S}} G\left(\log _{3} \varepsilon^{-1}\right)$, which is exactly of order $\varepsilon^{2-D_{C S}}$ since the nonconstant periodic function $G$ in 3.219 is bounded away from 0 and $\infty$.

${ }^{134}$ Accordingly, the complement of the kernel in the set of all (nonremovable) singularities could be referred to as the set of "analytic singularities". In practice, it can be ignored because it is not expected to contribute (in a significant way) to the fractal tube formula for $(A, \Omega)$, although this remains to be proved in general.
} 
of a countably infinite set of geometric singularities (which are not poles of $\zeta_{A, \Omega}$ or of $\left.\widetilde{\zeta}_{A, \Omega}\right)$ and is dense in the whole critical line $\{\operatorname{Re}(s)=D\}$, where $D:=\overline{\operatorname{dim}_{B}}(A, \Omega)$ can be prescribed a priori in the interval $(0, N)$. However, only the geometric singularities will contribute to the associated fractal tube formulas 135

Several other examples are provided in LapRaZu1. In one of those examples, the kernel consists of a countable set of essential singularities and is shown to contribute to the fractal tube formulas on the same footing as mere poles of $\zeta_{A, \Omega}$. The remaining singularities do not contribute to the main term in the fractal tube formula; they may, however, contribute to the error term (the 'noise').

It is then immediate to extend the above notion of fractality by saying that an object (say, an $\operatorname{RFD}(A, \Omega)$ in $\mathbb{R}^{N}$ ) is fractal if it has at least one nonreal (geometric) singularity. The notions of fractality in dimension $d \in \mathbb{R}$, as well as of critical and subcritical fractality, are similarly extended.

In the author's opinion, a deep understanding of the scaling laws in mathematics and physics (among many other fields, including cosmology, computer science, economics, chemistry and biology), as well as of many aspects of dynamics and of fractal, spectral and arithmetic geometry, could be gained by pursuing this venue and extending the theory of complex dimensions by merging it with aspects of the theory of Riemann surfaces 136 and using the notion of $h$-Minkowski content and related notions for a variety of (admissible) gauge functions. The beginning of such a theory is provided in a joint work under completion, LapRaZu10.

As is suggested in Lap10, in the long term, a potentially far-reaching further extension of the theory of complex dimensions to multivariable fractal zeta functions and their analytic varieties of singularities, merged with aspects of the theory of complex manifolds and sheaf theory (see, e.g., Ebe and GunRos ), should be even more fruitful in this context; see also the end of $\$ 4.4 .2$ (and of \$4) below.

We close this subsection by discussing originally unexpected connections between hyperfractality and the spectra of fractal drums.

3.6.4. Maximal hyperfractals and meromorphic extensions of spectral zeta functions of fractal drums. The error estimates obtained in Lap1 for the spectral asymptotics of fractal drums (i.e., drums with fractal boundary) are, in general, best possible. (See the paragraph following (2.41), along with part $(a)$ of Remark 2.12.) They also imply that the (normalized) spectral zeta function $\zeta_{\nu}$ associated with a given fractal drum 137 can be meromorphically extended to the open right half-plane $\{\operatorname{Re}(s)>D\}$, where $D$ is the (upper) Minkowski dimension of the boundary of the fractal drum 138

\footnotetext{
${ }^{135}$ Simpler examples of this type can be obtained by considering the Cartesian product of two different self-similar (and lattice) Cantor sets with incommensurable oscillatory periods.

${ }^{136}$ See, e.g., Ebe], Schl] and beyond, in the spirit of Riemann's original broader intuition of Riemann surface.

${ }^{137}$ For the Dirichlet Laplacian with eigenvalue spectrum $\left(\lambda_{j}\right)_{j=1}^{\infty}$, where the eigenvalues are repeated according to their multiplicities, we let $\zeta_{\nu}(s):=\sum_{j=1}^{\infty} f_{j}^{-s}$, for all $s \in \mathbb{C}$ with $\operatorname{Re}(s)$ sufficiently large, where for each $j \geq 1, f_{j}:=\sqrt{\lambda_{j}}$ is the $j$-th frequency of the fractal drum (written in nonincreasing order and repeated according to multiplicity).

${ }^{138}$ That is, $D:=\operatorname{dim}_{B}(\partial \Omega, \Omega)$ in the notation of relative fractal drums.
} 
In LapRaZu1, §4.3.2] and LapRaZu8, it is shown that the construction of (transcendentally $\infty$-quasiperiodic) maximal hyperfractal RFDs $(\partial \Omega, \Omega)$, where $\Omega$ is a bounded open set in $\mathbb{R}^{N}$ (and hence, has compact boundary $\partial \Omega$ ), carried out in LapRaZu1, §4.6.1] and LapRaZu2-4 (as was briefly discussed earlier in this subsection), implies that the above right half-plane $\{\operatorname{Re}(s)>D\}$ is in general optimal (i.e., as large as possible among all open right half-planes to which $\zeta_{\nu}$ can be meromorphically continued). This is so for any possible value of the (upper) Minkowski dimension of the RFD $(\partial \Omega, \Omega)$; namely, for any $N \geq 1$ and for every $D \in(0, N)$.

This last result establishes an interesting new connection between (maximal) hyperfractality and the vibrations of fractal drums. In particular, for each of the maximally hyperfractal drums or RFDs $(\partial \Omega, \Omega)$ constructed in LapRaZu1,8, the half-plane of meromorphic convergence of their spectral zeta function $\zeta_{\nu}$ coincides with the half-plane of (absolute) convergence of their distance zeta function $\zeta_{\partial \Omega, \Omega}$ (or equivalently, since $D<N$ here, of their tube zeta function $\widetilde{\zeta}_{\partial \Omega, \Omega}$ ).

\section{Epilogue: From Complex Fractal Dimensions to Quantized Number Theory and Fractal Cohomology}

In Lap-vF2-4, Lap-vF7 and Lap7 was proposed a search for a 'fractal cohomology theory' that would be naturally associated with the theory of complex fractal dimensions (at the time, as developed for fractal strings and described in part in 92 , but now also extended as in LapRaZu1 and LapRaZu2-10 to the much broader higher-dimensional setting, as described in \$3), as well as help unify at a deeper level several important aspects of fractal geometry, number theory and arithmetic geometry. (See Lap-vF4, §12.3 and §12.4], along with Lap7, Chs. 4 and 5].)

In particular, from this perspective (see Lap-vF4, §12.4]), an analogy was developed between lattice self-similar geometries and finite-dimensional (algebraic) varieties over finite fields, while nonlattices self-similar geometries could be seen as a limiting case corresponding to infinite dimensional varieties (seemingly over a "field of characteristic one"). Indeed, the zeros and the poles of the scaling zeta function 139 of lattice self-similar strings are periodically distributed along finitely many vertical lines. The same is true of self-similar sprays (as well as, conjecturally, of self-similar sets) with "nice generators". (For the case of self-similar strings, see Example 2.9, and for the more general case of self-similar sprays, see $\$ 3.4 .10,140$

4.1. Analogy between self-similar geometries and varieties over finite fields. Recall that for a (smooth, projective, finite-dimensional, algebraic) variety $V$ over a finite field $\mathbb{F}_{q}$ (where $q=p^{m}$, with $m \in \mathbb{N}$ and the prime number $p$ being the underlying prime characteristic), the corresponding zeta function $\zeta_{V}$ is periodic with complex period $i \mathbf{p}$ (i.e., $\zeta_{V}(s)=\zeta_{V}(s+i \mathbf{p}$ ), for all $s \in \mathbb{C}$ ), where $\mathbf{p}:=2 \pi / \log q=2 \pi / m \log p$. Therefore, as $q=\# \mathbb{F}_{q} \rightarrow \infty$ (e.g., if we successively consider $V$ over the finite field extensions $\mathbb{F}_{q n}$, with $n \in \mathbb{N}$ increasing to $\infty$ ), then the 'oscillatory period' $\mathbf{p}$ tends to 0 .

\footnotetext{
${ }^{139} \mathrm{By}$ definition, the scaling zeta function of a self-similar string $\mathcal{L}$ coincides with the geometric zeta function of $\mathcal{L}$.

${ }^{140}$ We leave aside here the 'integer dimensions' since we use the scaling (rather than the fractal) zeta functions.
} 
Similarly, for a lattice string (or spray), the corresponding scaling zeta function $\zeta_{\mathfrak{s}}$ is periodic with complex period $i \mathbf{p}$ (where $\mathbf{p}:=2 \pi / \log r^{-1}$ is the oscillatory period and $r$ in $(0,1)$ is the multiplicative generator of the underlying scaling ratios), in the same sense as above. Furthermore, in the approximation of a nonlattice string (or spray) by a sequence of lattice strings (or sprays) with increasing oscillatory periods $\mathbf{p}_{n}$ (as described in detail in [Lap-vF4, Ch. 3, esp., $\left.\S \S 3.4-3.5\right]$ and briefly discussed in Example 2.9), so that $\zeta_{\mathfrak{s}}\left(s+i u \mathbf{p}_{n}\right)$ and $\zeta_{\mathfrak{s}}(s)$ are close for $u \in \mathbb{C}$ with $|u|$ not too large), we have $\mathbf{p}_{n} \rightarrow \infty$. In this sense, nonlattice strings (or sprays) satisfy $\mathbf{p}=\infty$ (or $\mathbf{p} \rightarrow \infty)$ and behave as though $r \rightarrow 1^{+}$and hence, as though the "underlying prime characteristic were equal (or tending) to 1", as was mentioned above 141

Moreover, for a (finite-dimensional) variety $V$ over $\mathbb{F}_{q}$ (really, over the algebraic closure $\overline{\mathbb{F}}_{q}$ of $\mathbb{F}_{q}$ ), the total number of vertical lines, along which the zeros and the poles of $\zeta_{V}$ (counted according to their multiplicities) are distributed is equal to $2 d+1$, where $d:=\operatorname{dim} V$ is the dimension of the variety. In addition, the zeros (respectively, poles) correspond to the even (respectively, odd) cohomology spaces 142

The aforementioned (Weil-type or étale) cohomology spaces played a key role in the proof of the Weil conjectures Wei1-3 (and, in particular, of the counterpart of the Riemann hypothesis) for curves over finite fields by A. Weil in loc. cit. and then, for higher-dimensional (but still finite-dimensional) varieties over finite fields, by P. Deligne in Del1-2 143

In the process, a certain map (on the underlying variety $V$ and induced by the self-map $x \mapsto x^{q}$ of $\mathbb{F}_{q}$ ), called the Frobenius morphism, also plays a central role, via the linear endomorphism $F$ it induces on the total cohomology space and called the Frobenius operator 144 The (graded or alternating) 'characteristic polynomial' of $F$ is then shown to coincide with the zeta function of $V$ (in the variable $z=q^{-s}$ ). Symbolically, and with $Z_{V}(z):=\zeta_{V}\left(q^{-s}\right)$, the Weil zeta function of the variety $V$, we have (ignoring multiplication by an unessential nowhere vanishing entire function)

$$
Z_{V}(z)=s-\operatorname{det}(I-z F)
$$

\footnotetext{
${ }^{141}$ In the case of a self-similar spray with multiple generators, the scaling zeta function has both zeros and poles; in fact, it is of the form $\zeta_{\mathfrak{s}}(s)=\sum_{k=1}^{K} g_{k}^{s} /\left(1-\sum_{j=1}^{J} r_{j}^{s}\right)$, where the positive numbers $g_{k}$ and scaling ratios $r_{j}$ are not necessarily assumed to be distinct. By definition, the lattice case then corresponds to the situation when the group generated by the distinct values of the $g_{k}$ 's and $r_{j}$ 's is of rank 1 (with generator denoted by $r$ and assumed to lie in $(0,1)$ ); the associated oscillatory period is then $\mathbf{p}:=2 \pi / \log r^{-1}$.

${ }^{142}$ In other words, the total cohomology space is naturally $\mathbb{Z}_{2}$-graded, with $\dot{0}$ corresponding to the zeros and $i$ corresponding to the poles, for this choice of grading.

${ }^{143}$ For a brief introduction to curves (or, more generally, varieties) over finite fields, as well as to the associated zeta functions and Weil conjectures (including $\mathrm{RH}$ in this context), we point out, for example, Dieu1, Katz, Oort, ParsSh1 and Lap7 App. B], along with the many references therein (or since), including [Art, [Has, Schm], Wei1-3], Gro1-4, Del1-2], Den1-6], Har1-3, CobLap1-2] and Lap10. Also, for relevant notions from algebraic geometry, we refer, e.g., to Hart.

${ }^{144} \mathrm{By}$ using the periodicity of $\zeta_{V}$ (i.e., by making the changing of variable $z:=q^{-s}$ ), one formally obtains a reduced (total) cohomology space, which is a finite-dimensional vector space. In fact, in Wei1-3], Del1-2] or Gro1-4, only finite-dimensional vector spaces (over the underlying field) are considered.
} 
where "s-det" stands for the graded (or super, Del3, Wein) determinant of $F$ over the (reduced) total cohomology space. It was later conjectured by C. Deninger in Den1-6] (see, especially, Den1-3]) that a similar procedure (but now involving, in general, possibly infinite dimensional cohomology spaces) could be used to deal with the Riemann zeta function $\zeta=\zeta(s)$ and other $L$-functions (in characteristic zero, for example, in the case of $\zeta$, over the field $\mathbb{Q}$ ).

Since the early to mid-1990s, the author's intuition has been that there should exist a suitable notion of "fractal cohomology" such that the nonlattice case (for self-similar geometries) would be analogous to the situation expected to hold for $\zeta$ (the latter being a very special case, however, one typical of arithmetic geometries and for which RH would hold). In Lap7, building on Lap-vF1-3 (see also Lap-vF4 §12.4]), it was conjectured that a (generalized) Polya-Hilbert operator [having for spectrum (the reciprocals of) the zeros and poles (i.e., the reciprocal of the divisor) of the underlying fractal or arithmetic zeta function] should exist in this context so that the analog of (4.1) would hold and could be rigorously established. Partially realizing this dream has required significantly building on another semi-heuristic proposal made in Lap-vF3-4] (regarding a so-called "spectral operator"), then the development in HerLap1-5 of quantized number theory (in the "real case"), followed by the development in CobLap1-2 of quantized number theory (in the "complex case") and the construction of a corresponding (generalized) Polya-Hilbert operator, along with the foundations of an associated fractal cohomology, in Lap10.

4.2. Quantized number theory: The real case. We begin by presenting (in a very concise form) aspects of the first version of quantized number theory (in the "real case"), as developed by Hafedh Herichi and the author in HerLap1-5] and in Lap8, based on a rigorous notion of the infinitesimal shift $\partial$ of the real line and of the corresponding spectral operator (as proposed heuristically in Lap-vF3-4; see, especially, [Lap-vF4, §6.3] and [HerLap1, Ch. 4]). In particular, the theory developed in HerLap1-5 explains how to "quantize" the Riemann zeta function $\zeta=\zeta(s)$ in this context in order to view the spectral operator $\mathfrak{a}$ (which sends the geometry of fractal strings onto their spectrum) as follows (see [HerLap1, §7.2]):

$$
\mathfrak{a}=\zeta(\partial),
$$

where $\zeta(\partial)$ is interpreted in the sense of the functional calculus for the unbounded normal operator $\partial=\partial_{c}$ (with $\partial=d / d t$, the differentiation operator) acting on a suitable complex Hilbert space $\mathbb{H}_{c}:=L^{2}\left(\mathbb{R}, e^{-2 c t} d t\right)$, the weighted $L^{2}$-space with respect to the (positive) weight function $w_{c}(t):=e^{-2 c t}$, defined for all $t \in \mathbb{R}$. (See HerLap1, Ch. 5] for the precise definition of $\partial$, including of its domain of definition, as well as for the proof of the normality of $\partial: \partial^{*} \partial=\partial \partial^{*}$ since it is shown in loc. cit. that the adjoint $\partial^{*}$ of $\partial$ is given by $\partial^{*}=2 c-\partial$.)

Here, the "dimensional parameter" $c \in \mathbb{R}$ is fixed, but enables us, in particular, to sweep out the entire critical strip $0<R e(s)<1$ (corresponding to the "critical interval" $0<c<1)$ as well as the half-plane of absolute convergence for $\zeta=\zeta(s)$, namely, the open half-plane $\operatorname{Re}(s)>1$ corresponding to the half-line $c>1$ ).

The (strongly continuous) semigroup of bounded linear operators of $\mathbb{H}_{c}$ generated by $\partial=\partial_{c}$ is given by $\left\{e^{-h \partial}\right\}_{h \geq 0}$ and acts as the semigroup of translations (or 
shifts) of the real line (see HerLap1, §6.3]) 145

$$
\left(e^{-h \partial}\right)(f)(u)=f(u-h), \quad \text { for all } f \in \mathbb{H}_{c},
$$

as well as for almost every $u \in \mathbb{R}$ and for every $h \geq 0$. In fact, formula (4.3) is also valid for $h \leq 0$ and hence, the real infinitesimal shift $\partial=\partial_{c}$ is the infinitesimal generator of the one-parameter group of operators $\left\{e^{-h \partial}\right\}_{h \in \mathbb{R}}$.

We note that the semigroup $\left\{e^{-h \partial}\right\}_{h \geq 0}$ is a contractive (respectively, expansive) semigroup for $c \geq 0$ (respectively, $c \leq 0$ ), while the group $\left\{e^{-h \partial}\right\}_{h \in \mathbb{R}}$ is a group of isometries if and only if $c=0$. Finally, since the adjoint of $\partial$ is given by $\partial^{*}=2 c-\partial$, as is shown in HerLap1 $\$$ \$5.3], the adjoint group $\left\{e^{-h \partial^{*}}\right\}_{h \in \mathbb{R}}$ coincides with $\left\{e^{-2 c h} e^{h \partial}\right\}_{h \in \mathbb{R}}$.

It is shown in [HerLap1, $\S 6.2$ ] that the spectrum, $\sigma(\partial)$, of the real infinitesimal shift $\partial=\partial_{c}$ is given by $L_{c}$, the vertical line going through $c \in \mathbb{R} 1146$

$$
\sigma(\partial)=L_{c}:=\{\operatorname{Re}(s)=c\} .
$$

Hence, $\partial$ is an unbounded (normal) operator, for any value of $c \in \mathbb{R}$. Further, in light of (4.2) and (4.4), one deduces from a suitable version of the spectral mapping theorem for unbounded normal operators given in HerLap1, App. E] that the spectrum, $\sigma(\mathfrak{a})$, of the spectral operator $\mathfrak{a}=\zeta(\partial)$ coincides with the closure of the range of $\zeta=\zeta(s)$ along the vertical line $L_{c}$ (see HerLap1, §9.1]) 147

$$
\sigma(\mathfrak{a})=c \ell\left(L_{c}\right)=c \ell(\{\zeta(s): \operatorname{Re}(s)=c\}) .
$$

It follows from (4.5) that, in light of the Bohr-Courant theorem BohCou, itself now viewed as a mere consequence of the universality of $\zeta$ among all (suitable) analytic functions on the right critical strip $\{1 / 2<\operatorname{Re}(s)<1\}$ (as established in Vor], that

$$
\sigma(\mathfrak{a})=\mathbb{C}, \quad \text { for every } c \in(1 / 2,1) .
$$

In particular, for any $c \in(1 / 2,1)$, the spectral operator is not invertible (in the usual sense of unbounded operators); see HerLap1, §9.2].

Next, we examine what happens when $c>1$ (which, as we recall and in light of the identity (4.5), corresponds to the half-plane of absolute convergence for $\zeta=\zeta(s)=\sum_{n=1}^{\infty} n^{-s}$, namely, the open half-plane $\left.\{\operatorname{Re}(s)>1\}\right)$; see HerLap1. Ch. 7] for the full details.

For $c>1$, the spectral operator $\mathfrak{a}=\zeta(\partial)$ can be represented by a norm convergent quantized (or operator-valued) Dirichlet series and a quantized (also

\footnotetext{
${ }^{145}$ For the general theory of strongly continuous semigroups of bounded linear operators and its applications, from different points of view, we refer, e.g., to Go, HilPh, JoLap, JoLapNie, Kat, [ReSi1-3] and [Ru2, Sc, Yo.

${ }^{146}$ For the spectral theory of unbounded operators in various settings, we refer, e.g., to DunSch, ReSi1, JoLap, Kat, Ru2, Sc, Yo. We note, however, that the references ReSi1] and $[\mathbf{S c}]$ are mostly limited to the spectral theory of unbounded self-adjoint (rather than normal) operators, as are many of the references focusing on the applications to quantum physics.

${ }^{147}$ If $c=1$, the unique (and simple) pole of $\zeta$, one must exclude $s=1$ from the line $L_{c}$ in interpreting 4.5). Alternatively, one can consider the extended spectrum of $\mathfrak{a}$, denoted by $\widetilde{\sigma}(\mathfrak{a})$ and defined by $\widetilde{\sigma}(\mathfrak{a}):=\sigma(\mathfrak{a})$ if $\mathfrak{a}$ is bounded and $\widetilde{\sigma}(\mathfrak{a}):=\sigma(\mathfrak{a}) \cup\{\infty\}$ otherwise, and view the meromorphic function $\zeta$ as a continuous function from $\mathbb{C}$ to the Riemann sphere $\widetilde{\mathbb{C}}:=\mathbb{C} \cup\{\infty\}$. Then, the closure in 4.5] is interpreted in the compact space $\widetilde{\mathbb{C}}$ instead of in the unbounded complex plane $\mathbb{C}$.
} 
norm convergent) Euler product:

$$
\begin{aligned}
\mathfrak{a}=\zeta(\partial) & =\sum_{n=1}^{\infty} n^{-\partial} \\
& =\prod_{p \in \mathcal{P}}\left(1-p^{-\partial}\right)^{-1},
\end{aligned}
$$

where $\mathcal{P}$ denotes the set of prime numbers. It follows from (4.7) that for every $c>1, \mathfrak{a}$ is invertible (in the strong sense of $\mathcal{B}\left(\mathbb{H}_{c}\right)$, the space of bounded linear operators on $\mathbb{H}_{C}$ ), and that its (bounded) inverse $\mathfrak{a}^{-1}$ is given by

$$
\mathfrak{a}^{-1}=\sum_{n=1}^{\infty} \mu(n) n^{-\partial}=\prod_{p \in \mathcal{P}}\left(1-p^{-\partial}\right),
$$

where $\mu=\mu(n)$ is the Möbius function, defined by $\mu(1)=1, \mu(n)=0$ if the integer $n \geq 2$ is not square-free, and $\mu(n)=k$ if $n \geq 2$ is the product of $k$ distinct primes. We stress that for $c>1$, all of the infinite series and infinite products in (4.7) and (4.8) are convergent in the operator norm (i.e., in the Banach algebra $\mathcal{B}\left(\mathbb{H}_{c}\right)$ ).

Moreover, for $0<c<1$ (i.e., "within" the critical strip $0<\operatorname{Re}(s)<1$ ), the spectral operator $\mathfrak{a}=\mathfrak{a}_{c}$ is represented (when applied to a state function $f$ belonging to a suitable dense subspace of $\mathbb{H}_{c}$, which is also an operator core for $\partial=\partial_{c}$ ) by the same (but now weakly convergent) operator-valued Dirichlet series and Euler product as in (4.7). A similar comment applies to $\mathfrak{a}^{-1}$, which is then also a possibly unbounded operator when $0<c<1$.

Therefore, as was conjectured in $\S 6.3 .2$ of Lap-vF3 and of Lap-vF4, but now in a very precise sense, the spectral operator $\mathfrak{a}=\zeta(\partial)$, which can be viewed as the quantized Riemann zeta function, has an operator-valued Euler product representation (as well as a convergent Dirichlet series, which was not conjectured to exist in Lap-vF3, Lap-vF4 ) that is convergent (in a suitable sense) even in the critical strip $0<\operatorname{Re}(s)<1$ (i.e., even when the dimensional parameter $c$ lies in the critical interval $(0,1))$; see HerLap1, §7.5].

One of the key results of HerLap1 is to provide various characterizations of the "quasi-invertibility" of the spectral operator $\mathfrak{a}=\mathfrak{a}_{c} 148$ More specifically, the Riemann hypothesis $(\mathrm{RH})$ is shown to be equivalent to the fact that for $c \in(0,1)$, the spectral operator $\mathfrak{a}=\mathfrak{a}_{c}$ is quasi-invertible if and only if $c \neq 1 / 2$, thereby providing an exact operator-theoretic counterpart of the reformulation of $\mathrm{RH}$ obtained in LapMa2 (and briefly discussed in $\$ 2.6 .2$ ) in terms of inverse spectral problems for fractal strings. (See HerLap1, Ch. 8, esp., §8.3].)

Another, seemingly very different, reformulation of the Riemann hypothesis, obtained by the author in Lap8 (and also discussed in HerLap1, §9.4]) is the following statement: The Riemann hypothesis holds true if and only if the spectral operator $\mathfrak{a}=\mathfrak{a}_{c}$ is invertible (in the usual sense of unbounded operators) for every

\footnotetext{
${ }^{148}$ The possibly unbounded, normal operator $\mathfrak{a}=\zeta(\partial)$ is said to be quasi-invertible if for every $T>0$, the truncated spectral operator $\mathfrak{a}^{(T)}:=\zeta\left(\partial^{(T)}\right)$ is invertible, where $\partial^{(T)}:=\varphi^{(T)}(\partial)$ (defined via the functional calculus for unbounded normal operators) is the truncated infinitesimal shift and the function $\varphi^{(T)}$ is chosen so that $\sigma\left(\mathfrak{a}^{(T)}\right)=[c-i T, c+i T]$; see HerLap1 §6.4].
} 
$c \in(0,1 / 2) 149$ This result is referred to in $\mathbf{L a p 8}$ as an asymmetric criterion for $R H$.

Many other results are obtained in HerLap1-5] and Lap8, concerning, in particular, a quantized analog of the functional equation for $\zeta=\zeta(s)$ (or for its completion $\xi=\xi(s)$, see [HerLap1, $\S 7.6$ ] where the global spectral operator $\mathcal{A}=\mathcal{A}_{c}:=\xi\left(\partial_{c}\right)$ is studied) and of the universality of the Riemann zeta function KarVo, Lau, Steu (see HerLap1, Ch. 10, esp., §10.2]), as well as about the form of the inverse of the spectral operator, when it exists (see Lap8 and HerLap1 §9.4]), and the nature of the mathematical phase transitions at $c=1 / 2$ and at $c=1$ concerning the shape of the spectrum, the quasi-invertibility, the invertibility and the boundedness of $\mathfrak{a}=\mathfrak{a}_{c}$ (see HerLap1, §9.3]).

We note that the mathematical phase transitions occurring in HerLap1 at $c=1$ are analogous to (but different from) the one studied from an operatoralgebraic point of view by J.-B. Bost and A. Connes in BosCon1-2 and Con1, $\S \mathrm{V} . \mathrm{II}]$ (because the latter also corresponds to the pole at $s=1$ of $\zeta=\zeta(s)$ ), whereas the phase transitions occurring in HerLap1 and in [Lap8 in the midfractal case when $c=1 / 2$ are of a very different nature. They were expected to occur in the author's early conjecture (and open problem) formulated in Lap3 Quest. 2.6, p. $14]$ about the existence of a notion of complex fractal dimensions that would enable us to interpret the Riemann hypothesis as a phase transition in the midfractal case, in the sense of Wilson's work Wils on critical phenomena in condensed matter physics and quantum field theory.

For a full exposition of these results, we refer the interested reader to the book HerLap1. We note that the above results could likely be extended to a large class of meromorphic functions (instead of just the Riemann zeta function), and especially of the arithmetic $L$-functions ([Sarn, [Murt1-3, Lap7, App. C]) for which the counterpart of the Riemann hypothesis or of the universality theorem is expected to hold. In particular, conjecturally, the results concerning RH should have an appropriate counterpart for all the elements of the Selberg class (Sel1, Sarn, Murt1-3] and [Lap7, App. E]).

4.3. Quantized number theory: The complex case. We continue this epilogue by providing an extremely brief overview of the results of Tim Cobler and the author on "quantized number theory" (in the "complex case") obtained in CobLap1-2 and pursued in the book in preparation Lap10, in which the real infinitesimal shift $\partial=d / d t$ acting on $\mathbb{H}_{c}=L^{2}\left(\mathbb{R}, e^{-2 c t} d t\right.$ ) (with $c \in \mathbb{R}$ and discussed in 4.2 is replaced by the complex infinitesimal shift $\partial=d / d z$, now acting on a suitable weighted Bergman space $\mathcal{H}$ of entire functions (as introduced originally and for completely different purposes by A. Atzmon and B. Brive in AtzBri]). Namely, $\mathcal{H}$ consists of the entire functions belonging to the complex weighted Hilbert space $L^{2}\left(\mathbb{C}, e^{-|z|^{\alpha}} d z\right)$, for some fixed, but otherwise unimportant, parameter $\alpha \in(0,1) 150$

\footnotetext{
${ }^{149}$ By using a result in GarSteu, this is shown to be equivalent to the non-universality of $\zeta=\zeta(s)$ in the left critical strip $\{0<R e(s)<1 / 2\}$. On the other hand, in light of (4.5), the universality of the Riemann zeta function $\zeta=\zeta(s)$ in the right critical strip $\{1 / 2<\operatorname{Re}(s)<1\}$ (due to S. M. Voronin in Vor] and extended by B. Bagchi and A. Reich in Bag and Rei1-2]) implies that $\mathfrak{a}=\mathfrak{a}_{c}$ is never invertible for any $c \in(1 / 2,1)$.

${ }^{150}$ For the theory of (not necessarily weighted) Bergman spaces, see, e.g., DureSchu and HedKonZhu.
} 
For these values of $\alpha$, the operator $\partial$ is bounded (but not normal) and its spectrum, $\sigma(\partial)$, is given by a single point, namely, the origin 151

$$
\sigma(\partial)=\{0\} .
$$

From this, it follows that in the notation introduced below (namely, with $\partial_{\tau}:=$ $\partial+\tau)$, we have that $\sigma\left(\partial_{\tau}\right)=\{\tau\}$, for every $\tau \in \mathbb{C}$. We note that 0 is an eigenvalue of $\partial$ (in fact, its only eigenvalue) and that the associated eigenfunction is the (possibly suitably normalized) constant function 1 , the 'vacuum state'. With 0 replaced by $\tau \in \mathbb{C}$, the same statement holds for the shifted differentiation operator $\partial_{\tau}$.

The complex infinitesimal shift $\partial$ is the infinitesimal generator of the group of translations (or shifts) on the complex plane, $\left\{e^{-z \partial}\right\}_{z \in \mathbb{C}}$, acting on every $\psi \in \mathcal{H}$ as follows (for almost every $u \in \mathbb{C}$ and every $z \in \mathbb{C}$ ):

$$
\left(e^{-z \partial}\right)(\psi)(u)=\psi(u-z) .
$$

In CobLap1 are rigorously constructed "generalized Polya-Hilbert operators" (GPOs, in short), in a sense close to that of [Lap7], and infinite dimensional regularized determinants of the restrictions to their eigenspaces which enable one, in particular, to recover (under appropriate hypotheses) the corresponding meromorphic functions (or zeta functions) as suitable (graded or alternating) "characteristic polynomials" of the restrictions of the GPOs to their total eigenspaces, in the spirit of 4.1 (but well beyond).

More specifically, by working with the family of translates $\left\{\partial_{\tau}\right\}_{\tau \in \mathbb{C}}$ of the complex infinitesimal shift $\partial=d / d z$ (i.e., $\partial_{\tau}:=\partial+\tau$, for every $\tau \in \mathbb{C}$ ) and the associated Bergman spaces, one can then construct (via orthogonal direct sums) a kind of 'universal Polya-Hilbert operator' (GPO), $\mathbb{D}$, acting on a typically countably infinite direct sum $\mathcal{H}$ of Bergman spaces, whose spectrum (when it is discrete) consists only of (isolated) eigenvalues with finite multiplicities and coincides with any prescribed discrete subset of $\mathbb{C}$, for example, a (multi)set of complex dimensions or (the reciprocal of) the divisor [viewed as a $\mathbb{Z}_{2}$-graded (multi)set of zeros and poles] of a suitable meromorphic function, such as (an appropriate version of) the global Riemann zeta function.

We can then write the given meromorphic function $g=g(s)$ (assumed to be a quotient of two entire functions of finite orders) as a (typically infinite dimensional) regularized $\mathbb{Z}_{2}$-graded (or supersymmetric) determinant:

$$
g(s)=s-\operatorname{det}(I-s \mathcal{F}),
$$

where $\mathcal{F}=\mathcal{F}_{g}$ is the restriction of the GPO $\mathbb{D}$ to its total eigenspace; so that

$$
\sigma(\mathbb{D})=\sigma(\mathcal{F})=\mathfrak{D}(g)^{-1},
$$

the reciprocal of the divisor of $g$, in the sense of Definition 3.18, and the (graded) spectra of $\mathbb{D}$ and $\mathcal{F}$ are discrete and consist only of eigenvalues, which are the reciprocals of the zeros and the poles of $g=g(s)$ repeated according to their multiplicities.

The operator $\mathcal{F}=\mathcal{F}_{g}$ occurring in (4.11) and (4.12) and defined as the restriction of the GPO $\mathbb{D}=\mathbb{D}_{g}$ to its total eigenspace is called (in Lap10 ) the generalized Frobenius operator (GFO, in short) associated with $g$. Indeed, in the special case

${ }^{151}$ If we were to allow the value $\alpha=1$, then in (4.9), $\sigma(\partial)$, the spectrum of $\partial$, would be the closed unit disk (with center the origin) in $\mathbb{C}$ instead of being reduced to $\{0\}$. 
of varieties over finite fields $\mathbb{F}_{q}$ discussed in 4.1 it is clearly a version (in the $s$ variable) of the usual Frobenius operator $F$ (defined by means of the $z$-variable, with $z:=q^{-s}$ ) induced by the Frobenius morphism on the variety and acting on the underlying total cohomology space (which also coincides with the total eigenspace of $F$ ).

We stress that here and thereafter, $\mathbb{D}=\mathbb{D}_{g}$ is the GPO associated with the discrete (and $\mathbb{Z}_{2}$-graded) multiset $\mathfrak{D}(g)^{-1}$ consisting of 0 (if 0 is either a zero or a pole of $g$ ) and of the reciprocals of the (nonzero) zeros and poles of $g$. Under the aforementioned conditions on $g$ (which are relaxed in CobLap2), this choice of the GPO $\mathbb{D}_{g}$ guarantees the compactness (and the normality) of the GFO $\mathcal{F}_{g}$ and even, that $\mathcal{F}_{g}$ belongs to some Schatten class (i.e., the sequence of characteristic eigenvalues of $\mathcal{F}_{g}$ belongs to $\ell^{n}=\ell^{n}(\mathbb{C})$, for some integer $n \geq 1$ ); so that the regularized determinant in (4.11) is well defined (and is of order $n$ ).

The regularized superdeterminant (or Berezinian, in the terminology of supersymmetric quantum field theory; see, e.g., Del3, Wein) arising in (4.11) above can be written as a quotient of regularized determinants which are natural generalizations of the well-known (infinite dimensional) Fredholm determinants of trace class compact operators Fred. For a detailed exposition and for the genesis of the theory of these generalized Fredholm determinants, we refer to Sim1-3; see also CobLap1-2 and Lap10. We simply mention that it relies in part on the work in Fred], Plem, Poin5, Smit, Lids, GohKre and Sim1-3.

In the special case of the zeta function of a variety $V$ over a finite field $\mathbb{F}_{q}$ and after having made the change of variable $z:=q^{-s}$, we obtain a rational function $Z_{V}=Z_{V}(z)$ which can be written as an alternating product of (finite-dimensional) determinants, as in (4.1) in the setting of the Weil conjectures.

Furthermore, in the other very important special case when $g$ is an appropriate version of the completed (or global) Riemann zeta function, for example, $g(s)=$ $\xi(s):=\pi^{-s / 2} \Gamma(s / 2) \zeta(s)$ or $g(s)=\Xi(s):=(s-1) \xi(s)$, the corresponding regularized determinant is then truly infinite dimensional since $\zeta=\zeta(s)$ (and hence also $\xi=$ $\xi(s)$ as well as $\Xi=\Xi(s)$ ) has infinitely many (critical) zeros. Moreover, the regularized determinant corresponding to the zeros is not a mere Fredholm operator Fred but involves a renormalization of second order (that is, in the hierarchy of such regularized determinants indexed by integers $n \geq 1$, as in [Sim2], it is of level $n=2)$.

These results provide, in particular, a partial but completely rigorous and quite general mathematical realization of what was sought for by a number of authors, including A. Weil Wei1-6, A Grothendieck Gro1-4, P. Deligne Del1-2, and especially, in the present context, C. Deninger [Den1-6]. Naturally, we should point out that they do not provide a full realization of those authors and their successors' rich set of ideas and conjectures, especially concerning the existence of suitable cohomology theories (Wei1-3, Gro1-4, [Den1-6] and, e.g., Tha1-2, along with the relevant references therein) and of an appropriate positivity condition (Wei4-6, Har1-3, Con2), and therefore cannot (for now) be used to try to prove the Riemann hypothesis, although they may constitute a significant first step, particularly once (and if) one can obtain an appropriate geometric and topological (i.e., cohomological) interpretation (which may, however, be very difficult and take a very long time to achieve; see 4.4 . 
We also mention that the search for suitable Polya-Hilbert operators has been the object of a number of works, both in physics (see, e.g., [Berr3-4] and BerrKea]) and in mathematics (see, e.g., Den1-5, Con2 and [Lap7, Chs. 4 \& 5]). The abstract (functional analytic) framework for GPOs provided in CobLap1-2 and extended in Lap10 is, in some ways, too general for certain purposes (for example, for proving $\mathrm{RH}$, at least in our current state of knowledge) but presents the advantage of being completely categorical and widely applicable, well beyond the settings of fractal geometry and number theory which originally motivated it.

The proof of the identity (4.11) given in CobLap1 makes use of Hadamard's factorization theorem for entire functions of finite order ([Had1-2], Conw $)$.

As is shown in Lap10, the above formalism (and, in particular, the identity (4.11)) can also be applied to all the elements of the Selberg class ([Sel2, Sarn, Murt1-3 and [Lap7, App. E]) and hence, at last conjecturally, to all of the arithmetic zeta function or $L$-functions (more specifically, the automorphic $L$-functions) for which the extended Riemann hypothesis (ERH) is expected to hold (see, e.g., [Sarn, Murt1-3, ParsSh1-2] and Lap7, App. C]). In this case, one also needs to use regularized determinants of order two, as for the various versions of the completed Riemann zeta function discussed above.

4.4. Towards a fractal cohomology. Here, we briefly discuss the emerging theory of 'fractal cohomology', as expounded upon in Lap10. This theory builds on the work from CobLap1 (and from CobLap2 described in $\$ 4.3$ itself inspired in part by the theory from HerLap1 described in 4.2 , as well as on the many references provided in $\$ 4.1$ including [Wei1-3, Gro1, Den3] and Lap-vF2-4.

The idea underlying the notion of a fractal cohomology is that to every complex dimension (now understood in the extended sense of a zero or a pole of the given fractal zeta function or arithmetic zeta function or else, more generally, of a suitable meromorphic function $g=g(s)) 152$ one can associate a finite-dimensional complex Hilbert space $H^{ \pm}(\omega)$, with the plus (respectively, minus) sign corresponding to $\omega$ being a zero (respectively, a pole) of dimension (over $\mathbb{C}$ ) equal to the multiplicity of $\omega$.

More generally, to (the reciprocal $\mathfrak{D}^{-1}(g)$ of) the divisor $\mathfrak{D}(g)$ of the meromorphic function $g$, we associate a $\mathbb{Z}_{2}$-graded (or supersymmetric) complex (and separable) Hilbert space 153

$$
H=H^{+} \oplus_{s} H^{-},
$$

where

$$
H^{+}:=\underset{\omega \in \mathcal{Z}^{+}}{\oplus} H^{+}(\omega) \quad \text { and } \quad H^{-}:=\underset{\omega \in \mathcal{Z}^{-}}{\oplus} H^{-}(\omega)
$$

with $\mathcal{Z}^{+}$(respectively, $\mathcal{Z}^{-}$) denoting the set of the reciprocals of the distinct zeros (respectively) poles of $g$ (with the convention according to which one does not have

\footnotetext{
${ }^{152}$ Note that the zeros and poles of the meromorphic function $g=g(s)$, can be viewed as the (necessarily simple) poles of (minus) the logarithmic derivative of $g$, the function $-g^{\prime} / g$, with the associated residues being equal to the orders of the zeros or of the poles (and with corresponding signs identifying whether the poles of $-g^{\prime} / g$ comes from a zero or a pole of $g$ ).

${ }^{153}$ Here and thereafter, for notational simplicity, we use the same symbol $\oplus_{s}$ to indicate the $\mathbb{Z}_{2}$-graded (or supersymmetric) direct sum of Hilbert spaces or of operators acting on them, as well as of (multi)sets, depending on the context.
} 
to take the reciprocal of 0 , if this value occurs in either $\mathcal{Z}^{+}$or $\mathcal{Z}^{-}$). The finitedimensional (complex) Hilbert space $H^{ \pm}(\omega)$ has dimension equal to the multiplicity of the corresponding pole or zero $\omega$ of $g=g(s)$.

As was explained in $4.3, H$ is the eigenspace of the generalized Polya-Hilbert space $(\mathrm{GPO}) \mathbb{D}=\mathbb{D}_{g}=\mathbb{D}_{\mathcal{Z}}$, with $\mathcal{Z}:=\mathcal{Z}^{+} \oplus_{s} \mathcal{Z}^{-}$and $\mathbb{D}=\mathbb{D}^{+} \oplus_{s} \mathbb{D}^{-}$. Furthermore, $\mathcal{F}=\mathcal{F}^{+} \oplus_{s} \mathcal{F}^{-}$, defined as the restriction of the GPO $\mathbb{D}=\mathbb{D}^{+} \oplus_{s} \mathbb{D}^{-}$to its (total) graded eigenspace $H=H^{+} \oplus_{s} H^{-}$(as given by (4.13) and (4.14)). The (possibly unbounded) operator $\mathcal{F}$ is called the generalized Frobenius operator (GFO) associated with $g$ (or with $\mathcal{Z}$ ). Under suitable assumptions on $g$ (specified in CobLap1-2 and in Lap10 154 the GFO $\mathcal{F}$ enables us to recover the meromorphic function $g$ via the determinant formula (4.11) showing that $g=g(s)$ is the "characteristic polynomial" of $\mathcal{F}$ (viewed as a supersymmetric regularized determinant).

REMARK 4.1. By construction, the multiplicity of $\omega \in \mathcal{Z}^{+}$(respectively, $\omega \in$ $\mathcal{Z}^{-}$), with $\omega \neq 0$, as an eigenvalue of the GPO $\mathbb{D}^{+}$(respectively, $\mathbb{D}^{-}$), or equivalently, of the GFO $\mathcal{F}^{+}$(respectively, $\mathcal{F}^{-}$), coincides with the multiplicity of the corresponding zero (respectively, pole) $\omega^{-1}$ of the meromorphic function $g=g(s)$. Hence, the finite-dimensional complex Hilbert space $H(\omega)$ has dimension over $\mathbb{C}$ equal to the multiplicity of the corresponding zero (respectively, pole) $\omega^{-1}$ of $g=g(s) 155$ Consequently, since finite-dimensional Hilbert spaces of the same dimension are isomorphic, the (total) cohomology space $H$ in (4.13) could be equivalently defined by letting $\omega$ run through the divisor $\mathfrak{D}(g)$ of $g$ (rather than through its reciprocal $\mathfrak{D}^{-1}(g)$, as indicated in (4.14) $)$ : symbolically,

$$
H=\underset{\omega \in \mathfrak{D}(g)}{\oplus} H(\omega) .
$$

Accordingly, in (4.14), the "even" (respectively, "odd") cohomology space $H^{+}$(respectively, $H^{-}$) can be defined by replacing $\mathcal{Z}^{+}$(respectively, $\mathcal{Z}^{-}$) by the set of distinct poles (respectively, of distinct zeros) of $g=g(s)$ : symbolically,

$$
H^{+}=\underset{\omega \in\{\text { zeros of } g\}}{\oplus H^{+}(\omega)} \quad \text { and } \quad H^{-}=\underset{\omega \in\{\text { poles of } g\}}{\oplus H^{-}} .
$$

When $\mathcal{Z}$ is infinite (that is, when $g$ has at least infinitely many zeros or poles), then the total cohomology space $H=H_{g}=H_{\mathcal{Z}}$ is infinite dimensional, and vice versa. Furthermore, since $\mathcal{Z}$ is at most countable, the cohomology space $H$ is a separable (complex) Hilbert space. This is the case, for example, when $g=g(s)$ is one of the completed Riemann zeta functions, $\xi=\xi(s)$ or $\Xi=\Xi(s)$. Therefore, the (total) cohomology space $H_{\xi}$ or $H_{\Xi}$ is intrinsically infinite dimensional and cannot be reduced to a finite-dimensional one.

On the other hand, in the case of the zeta function attached to a variety $V$ over a finite field $\mathbb{F}_{q}$ (as in 4.1), the periodicity of the zeta function combined with the change of variable $z:=q^{-s}$ yields a rational function $Z_{V}=Z_{V}(z)$ to which is associated a finite-dimensional cohomology space (corresponding to the zeros and the poles of the rational function, counted according to their multiplicities). Hence,

\footnotetext{
${ }^{154}$ More specifically, in light of CobLap1, one assumes that the meromorphic function $g=f / h$ is the ratio of two entire functions $f$ and $h$ of finite orders (which is sufficient for all of the potential applications to number theory, as well as for many of the potential applications to fractal geometry), while according to CobLap2, one should be able to remove the hypothesis that the entire functions $f$ and $h$ are of finite orders.

${ }^{155}$ Of course, the same is true if $\omega=0$ provided we replace $\omega^{-1}$ by $\omega$, in the latter statement.
} 
in this case, the total cohomology space is a priori infinite dimensional but can also naturally be reduced to a finite-dimensional one.

We thus obtain a cohomology theory which seems to satisfy several (if not all) of the main properties expected by Grothendieck (in Gro1-4) and by Deninger (in Den1-6]), for example. (See also, e.g., Tha1-2 for an exposition of some of those ideas.) Let us further explain this statement, but without going into the details. The key is that this "fractal cohomology theory" satisfies the counterpart (in the present context) of the Künneth formula (for the product of two algebraic varieties or of two differentiable manifolds; see, e.g., Dieu1,2 and MacL). This is, in fact, a key motivation for the author's conjecture (Conjecture 3.16) made in $\$ 3.4$ about the set of complex dimensions (really, the divisor of the associated fractal zeta function) of the Cartesian product of two bounded sets and, more generally, of two RFDs (in $\mathbb{R}^{N}$ ). If correct, this conjecture would help provide a geometric interpretation of the Künneth formula of fractal cohomology theory in terms of Cartesian products (at least in the geometric setting of bounded sets and RFDs in $\left.\mathbb{R}^{N}\right)$.

A suitable version of Poincaré duality (or of an appropriate extension thereof) in the context of fractal cohomology still remains to be found; see, e.g., Poin1-4, Dieu1,2 and MacL for the classic notions of Poincaré duality. A natural extension of that notion could clearly be formulated, however, in terms of the fractal cohomology spaces associated with $g(s)$ and with $g(1-s)$.

It is noteworthy that Grothendieck's beautiful dream and elusive notion of a "motive" is in fact intimately connected with the search for a "universal cohomology theory" satisfying several axioms, including especially, the analog of Künneth's formula and of Poincaré duality. (See, e.g., Kah, Tha1 and Tha2]. 156 The emerging fractal cohomology theory proposed in Lap10 (and building, in particular, on Lap-vF4], Lap7 and CobLap1,2]) is evolving in that spirit. It associates (in a functorial way) to a suitable meromorphic function $g$ (or to its divisor $\mathfrak{D}(g)$ ) a cohomology space $H_{g}$, which is the eigenspace of the generalized Frobenius operator $(\mathrm{GFO}) \mathcal{F}=\mathcal{F}_{g}$. In addition, the identity (4.11) which enables one (under appropriate hypotheses) to recover the meromorphic function from the action of $\mathcal{F}$ on $H$ can be viewed as naturally providing an associated inverse functor in this context.

4.4.1. Grading of the fractal cohomology by the real parts. It is natural to wonder why, unlike in standard algebraic or differential toplogy (see, e.g., Dieu2 and MacL), the fractal cohomology spaces are no longer (in our context) indexed by integers. In fact, a natural grading of the total fractal cohomology space $H=H^{+} \oplus_{s} H^{-}$is provided by the real parts (of the reciprocals) of the zeros and poles of the meromorphic function $g$ Hence, in general, $H$ is graded by real numbers and not just by (nonnegative) integers 158

More specifically, let $\mathcal{Z}=\mathcal{Z}^{+} \oplus_{s} \mathcal{Z}^{-}$, where $\mathcal{Z}^{+}$and $\mathcal{Z}^{-}$denote, respectively, the reciprocals of the distinct zeros and poles of $g$ (with the same convention concerning 0 as usual). Furthermore, let $R^{ \pm}$denote the (at most countable) set of real parts

\footnotetext{
${ }^{156}$ See also Cart for an interesting discussion of the evolution of the notion of a 'geometric space'.

${ }^{157}$ If 0 is either a zero or a pole of $g$, then $\mathrm{H}^{+}$or $\mathrm{H}^{-}$is also graded by zero.

${ }^{158}$ In light of Remark 4.1 we could replace throughout this discussion the real parts of the reciprocals of the zeros and of the poles by the zeros and the poles themselves; see also Remark 4.2
} 
of the elements of $\mathcal{Z}^{ \pm}$. Moreover, for $\alpha \in R^{ \pm}$, let

$$
\mathcal{Z}_{\alpha}^{ \pm}:=\left\{\omega \in \mathcal{Z}^{ \pm}: \operatorname{Re}(\omega)=\alpha\right\}
$$

and

$$
H_{\alpha}^{ \pm}:=\underset{\omega \in \mathcal{Z}_{\alpha}^{ \pm}}{\oplus} H(\omega)
$$

where, as earlier, $H(\omega)$ denotes the eigenspace corresponding to the eigenvalue $\omega$ of $\mathcal{F}_{g}$ (and also of $\mathbb{D}_{g}$ ); so that the dimension (over $\mathbb{C}$ ) of the finite-dimensional Hilbert space $H^{ \pm}(\omega)$ is equal to the multiplicity of $\omega$ as an eigenvalue of $\mathcal{F}_{g}$ (or, equivalently, if $\omega \neq 0$, as the reciprocal of a zero or a pole of $g$; if $\omega=0$, then we can omit the word "reciprocal" here).

Then, we can now provide the following precise formulation of the statement according to which the (total) fractal cohomology space is graded by the real parts, of the elements of $\mathfrak{D}(g)^{-1}$ :

$$
H^{ \pm}=\underset{\alpha \in R^{ \pm}}{\oplus} H_{\alpha}^{ \pm}
$$

with $R^{ \pm}$defined just before (4.17) and $H_{\alpha}^{ \pm}$defined by (4.18) for all $\alpha \in R^{ \pm}$.

REMARK 4.2. For notational simplicity, we have worked above with the grading provided by the real parts of the elements of $\mathcal{Z}^{+}$and $\mathcal{Z}^{-}$. However, in light of Remark 4.1. we could just as well replace $\mathcal{Z}^{+}$and $\mathcal{Z}^{-}$by the sets of distinct zeros and poles of $g=g(s)$, respectively. And similarly, for the definition of $R^{ \pm}, \mathcal{Z}_{\alpha}^{ \pm}, H_{\alpha}^{ \pm}$, $H$ and $H^{ \pm}$in (or around) (4.13), (4.14) and (4.17)-(4.19). Consequently, in that setting, the fractal cohomology spaces $H, H^{+}$and $H^{-}$would actually be indexed by the real parts of the elements of the divisor $\mathfrak{D}(g)$, the zero set and the pole set of the meromorphic function $g=g(s)$, respectively. Accordingly, the corresponding grading index sets $R^{+}$and $R^{-}$would instead consist of the real parts of the distinct zeros and of the distinct poles of $g=g(s)$, respectively.

We note that $H^{ \pm}$is graded by the (possibly infinite, BruDKPW] matroid

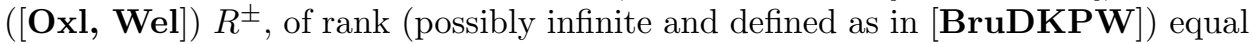
to the number of vertical lines in $\mathcal{Z}^{ \pm}$(or, equivalently, to the cardinality of $R^{ \pm}$, viewed either as the set of distinct zeros/poles of $g=g(s))$.

For example, for a $d$-dimensional variety $V$ over a finite field $\mathbb{F}_{q}, R^{-}$(respectively, $R^{+}$) consists of the integers $j$ (respectively, the half-integers $j / 2$ ), where $j \in\{0,1, \cdots, d\}$. This is the case relative to the original $s$-variable. On the other hand, if $z:=q^{-s}$, then the corresponding sets $R^{-}$and $R^{+}$are $\left\{q^{-j}: j=0,1, \cdots, d\right\}$ and $\left\{q^{-j / 2}: j=0,1, \cdots, d\right\}$, respectively. Thus, in light of the analog of the Riemann hypothesis proved by Deligne in Del1-2 in the general case when $d \geq 1$ (and by Weil in [Wei1-3] when $d=1$ ), the determinant formula (4.11) yields (still in the $z$-variable)

$$
Z_{V}(z)=\frac{Q_{1}(z) \cdots Q_{2 d-1}(z)}{Q_{0}(z) \cdots Q_{2 d}(z)}
$$

where for $k \in\{0, \cdots, d\}$, the polynomial $Q_{2 k}$ is the characteristic polynomial of the linear operator $\mathbf{F}_{2 k}:=\mathcal{F}_{q-k}^{-}$(the restriction of the GFO $\mathcal{F}$ to $\mathbf{H}_{2 k}:=H_{q-k}^{-}$) and for $k \in\{1, \cdots, d\}$, the polynomial $Q_{2 k-1}$ is the characteristic polynomial of the linear operator $\mathbf{F}_{2 k-1}:=\mathcal{F}_{q^{-k / 2}}^{+}$(the restriction of the GFO $\mathcal{F}$ to $\mathbf{H}_{2 k-1}:=H_{q^{-k / 2}}^{+}$). With this notation, (4.19) becomes

$$
H^{+}=\underset{k=1}{\oplus} \mathbf{H}_{2 k-1} \quad \text { and } \quad H^{-}=\underset{k=0}{\oplus} \mathbf{H}_{2 k},
$$


while the total cohomology space $H$ is given by

$$
H=H^{+} \oplus_{s} H^{-}=\underset{j=0}{\stackrel{2 d}{\oplus} \mathbf{H}_{j} .}
$$

In the case of the completed Riemann zeta function $\xi(s)$, and assuming the truth of the classic Riemann hypothesis, we have that $R^{+}:=\{1 / 2\}$ and $R^{-}:=\{0,1\}$, while $\mathcal{Z}^{-}=\{0,1\}$ and

$$
\mathcal{Z}^{+}:=\{\text {reciprocals of the critical zeros of } \zeta(s)\} .
$$

Hence, with the notation 159

$$
\mathbf{H}_{0}:=H_{0}^{-}, \quad \mathbf{H}_{2}:=H_{1}^{-}, \quad \text { and } \quad \mathbf{H}_{1}:=H_{1 / 2}^{+},
$$

while $\mathcal{F}_{j}$ is defined as the restriction of the generalized Frobenius operator $\mathcal{F}$ to $\mathbf{H}_{j}$, for $j \in\{0,1,2\}$. Then, (4.11) becomes (neglecting an unimportant factor) 160

$$
\xi(s)=\frac{\operatorname{det}\left(I-s \mathcal{F}_{1}\right)}{\operatorname{det}\left(I-s \mathcal{F}_{0}\right) \operatorname{det}\left(I-s \mathcal{F}_{2}\right)},
$$

with $\operatorname{det}\left(I-s \mathcal{F}_{0}\right)=s$ and $\operatorname{det}\left(I-s \mathcal{F}_{2}\right)=s-1$ corresponding to the pole of $\xi=\xi(s)$ at $s=0$ and at $s=1$, respectively.

Note that with the above notation (which is inspired by the one used for varieties over finite fields as well as by what is expected for Weil-type cohomologies in the present context as well as for more general arithmetic $L$-functions) 161 we have (in light of (4.24) )

$$
H^{+}=\mathbf{H}_{1} \quad \text { and } \quad H^{-}=\mathbf{H}_{0} \oplus \mathbf{H}_{2} .
$$

The classic Riemann hypothesis (for $\zeta$ or, equivalently, for $\xi$ ) is equivalent to the fact that the total even cohomology space of $\xi=\xi(s)$ (namely, $H^{+}=H_{\xi}^{+}=\mathbf{H}_{1}$ ) is 'monofractal'; i.e., with the notation of Remark 4.2. $\mathrm{RH}$ is equivalent to the fact that $R^{+}$consists of a single point (of necessity, then, $R^{+}=\{1 / 2\}$ ) 162

Entirely analogous statements can be made about the zeta functions of number fields ParsSh1-2] and of more general arithmetic $L$-functions, such as automorphic $L$-functions or conjecturally equivalently (see, e.g., Lap7, App. C and App. E]), the members of the Selberg class.

In contrast, the total odd cohomology space $H^{-}=H_{\zeta_{\mathcal{L}}}^{-}$(still relative to the underlying $\mathbb{Z}_{2}$-grading) of a generic nonlattice self-similar string $\mathcal{L}$ is 'multifractal' in a very strong sense; namely, $R^{-}$, the set of real parts of the complex dimensions of $\mathcal{L}$ (in the usual sense of $₫ 2$ and $\Upsilon_{3}$ ) is a countably infinite set which is dense in a compact interval $\left[D_{\ell}, D\right]$, where $-\infty<D_{\ell}<D$ and $D=D_{\mathcal{L}}$ is the Minkowski

\footnotetext{
${ }^{159}$ We are now using the grading of the total cohomology space by the real parts of the zeros and the poles of $\xi=\xi(s)$, as in Remark 4.2

${ }^{160}$ This factor is a nonvanishing entire function involving the number $\pi$ and Euler's constant $\gamma$; see CobLap1 and Lap10 for its specific value.

${ }^{161}$ See, e.g., Gro1-3], Dieu1, Den3, Con2], Lap7, esp., Ch. 4 \& App. B], Kah, Tha1-2 and the relevant references therein.

${ }^{162}$ Observe that by the functional equation for $\xi$ (i.e., $\xi(s)=\xi(1-s)$, for all $s \in \mathbb{C}$ ) and since $\xi$ has zeros on the critical line $\{\operatorname{Re}(s)=1 / 2\}$, we must have $R^{+}=\{1 / 2\}$ since otherwise, $R^{+}$ would contain at least three points, $1 / 2, \rho$ and $1 / 2-\rho$, for some $\rho \in(0,1 / 2)$. Also, conversely, if $R^{+}$had more than one point, then $\mathrm{RH}$ would obviously be violated.
} 
dimension of $\mathcal{L} 163$

4.4.2. Open problems and perspectives: Geometric interpretation and beyond. We close this section (and paper) by mentioning a few open problems and longterm research directions related to the nascent fractal cohomology theory and its possible geometric and topological interpretations:

(i) Geometric interpretation. It would be very useful and interesting to obtain a good geometric interpretation of the theory. It is natural to wonder what is the 'curve' (or 'variety') underlying fractal cohomology. By analogy with the case of curves (or, more generally, varieties) over finite fields, one may guess that the underlying supersymmetric 'fractal curve' is the divisor $\mathfrak{D}(g)$ of the given meromorphic function $g$ (or perhaps, its inverse, $\mathfrak{D}(g)^{-1}$, as defined earlier) 164 Ignoring the distinction between $\mathfrak{D}(g)$ and its inverse, let us denote symbolically this 'curve' by $C=C_{g}$. Then, abusing geometric language, one can think of the original Hilbert space $\mathcal{H}_{g}$, the eigenspace of the GPO $\mathbb{D}_{g}$ (namely, the fractal cohomology space $H_{g}$ ) and even the GPO $\mathbb{D}_{g}$ and the GFO $\mathcal{F}_{g}$, as 'bundles' or 'sheaves' over $C$.

If we let the function $g$ vary in a suitable 'moduli space' of meromorphic functions (or zeta functions) 165 we then obtain geometric, analytic and algebraic structures (such as $C_{g}, \mathcal{H}_{g}, \partial_{g}, \mathbb{D}_{g}, \mathcal{F}_{g}$ and $H_{g}$ ) which are naturally defined on that moduli space.

Pursuing the analogy with curves (or, more generally, varieties) over finite fields, one may wonder if the curve $C$ should not be augmented and replaced by a new (and larger) 'curve' $\widetilde{C}$ whose (generic) points would coincide with the fixed points of the generalized Frobenius operator $(\mathrm{GFO}) \mathcal{F}$ and of its iterates $\mathcal{F}^{n}$ (with $n \in \mathbb{N}$ arbitrary). In the present discussion, we allow the solutions (i.e., the fixed points of $\mathcal{F}^{n}$, for some $n \in \mathbb{N}$ ) to be distributional solutions (in a suitably defined weighted space of tempered distributions) and not just the Hilbert space solutions 166 Then, a simple differential equation computation (see Lap10 ) shows that $\widetilde{C}=\widetilde{C}_{g}$ consists of the points of $C=C_{g}$ augmented by a copy of the group $\mu(\infty)$ of all (complex) roots of unity attached to (and acting on) each point of $C 167$ In other words, $\widetilde{C}$ can be viewed as a 'principal bundle' over $C$, with structure group $\mu(\infty)$. Alternatively, $\widetilde{C}$ can be viewed as a 'sheaf' over $C$.

Amazingly and likely not coincidentally, the group $\mu(\infty)$ (and variants thereof) also plays a key role in the theory of motives over the elusive field of one element,

${ }^{163}$ We assume here implicitly that there are no (drastic) cancellations between the zeros and the poles of $\zeta_{\mathcal{L}}$. If we work instead with $H^{-}=H_{\partial \Omega, \Omega}^{-}$, where the $\operatorname{RFD}(\partial \Omega, \Omega)$ is a geometric realization of $\mathcal{L}$, then $R \backslash\{0\}$ is of the above form.

${ }^{164}$ For a nonlattice self-similar string $\mathcal{L}$ (in the case of a single gap), according to the results obtained in Lap-vF4, Ch. 3, esp., §3.4], the (multi)set of complex dimensions $\mathfrak{D}(g)=\mathcal{D}\left(\zeta_{\mathcal{L}}\right)$ obeys a quasiperiodic pattern and is conjectured to form a 'generalized quasicrystal' (in a sense to be yet fully specified); see Lap-vF4 Problem 3.22]. More broadly, in Lap7] esp., Ch. 5 and App. F], this type of generalized quasicrystal plays a key conceptual role.

${ }^{165}$ Compare with the moduli spaces of fractal membranes (and of the associated zeta functions or spectral 'partition functions') that play a central role in [Lap7 Ch. 5].

${ }^{166}$ If we only allowed Hilbert space solutions, then $\widetilde{C}$ would simply coincide with $C$.

${ }^{167}$ Hence, $\mu(\infty)=\cup_{n \geq 1} \mu(n)$, where for each $n \in \mathbb{N}, \mu(n)$ is the group of (complex) $n$-th roots of unity; clearly, here, $\mu(n)$ does not denote the value of the Möbius function at $n$. 
$\mathbb{F}_{1}$ (which originated in Y. Manin's seminal article Mani]); see, especially, $\mathbf{K a h}$, [Tha1], Tha2 and the relevant references therein 168

In the aforementioned references, working over $\mathbb{F}_{1}$ typically leads to adding new points to the underlying curve or variety (or motif) that were formally invisible. The same is true in the present situation of 'fractal motives' and the associated fractal cohomology. Indeed, going from $C$ to $\widetilde{C}$ amounts to adding previously invisible points. Analytically, as was alluded to above, this amounts to going outside the original Hilbert space $\mathcal{H}_{g}$ and finding all of the fixed points of the iterates $\mathcal{F}_{g}^{n}$ (for any $n \geq 1$ ) that lie in a suitable weighted space of tempered distributions containing $\mathcal{H}_{g}$; see Lap10.

In this context, for every given $n \in \mathbb{N}$, the fixed points of $\mathcal{F}^{n}$, correspond to the 'points' of the curve $\widetilde{C}$ which lie in the $n$-th field extension of $\mathbb{F}_{1}$. Heuristically, they can be thought of as a 'principal bundle' over $C$ with structure group $\mu(n)$, the group of $n$-th complex roots of unity. (Note the close analogy with the case of curves (or varieties) over finite fields discussed in 84.1 .)

Therefore, at the most fundamental level, we should aim at interpreting the present theory of fractal cohomology (as developed in CobLap1-2 and Lap10) as a universal cohomology theory of 'fractal motives' over $\mathbb{F}_{1}$, the elusive field of one element. Finding a coherent and completely rigorous geometric and arithmetic interpretation of this type is clearly a long-term open problem.

Remark 4.3. The 'curve' $C=C_{g}$ itself, or its augmentation (by invisible points, the distributional fixed points of $\mathcal{F}^{n}$, for any $\left.n \in \mathbb{N}\right) \widetilde{C}=\widetilde{C}_{g}$, can be viewed as the 'sheaf of complex dimensions' (here, visible zeros and poles of $g$ ) associated with 'the' analytic continuation of $g$ (i.e., with 'the' meromorphic continuation of $g$ on any given connected open set $U$ of $\mathbb{C}$ on which the latter exists). Interestingly, the notion of a sheaf was introduced in the 1940s by J. Leray to solve certain problems in cohomology theory and then used to precisely deal with the a priori ill-defined notion of analytic continuation of a function of several complex variables, in connection with the Cousin problem. (See, e.g., GunRos, Ebe and [Dieu2.) The previously mentioned extensions of the notion of complex dimensions (as nonremovable singularities of suitable complex analytic functions on Riemann surfaces, or in higher dimensions, on complex analytic varieties or even, on analytic spaces) and of the associated theory (see $\$ 2.5$ and $\$ 3.6$, as well as part (iii) of the present subsection) would, in the long term, provide a natural and significantly broader framework within which to consider the (yet to be precisely defined) sheaves $C$ and $\widetilde{C}$ in this extended context.

We next very briefly mention two other key research directions in this area:

(ii) Fractal homology. As is well known, standard cohomology theories in topology and in differential geometry, for example) are often the dual theories of suitable homology theories. (See, e.g., Dieu2 and MacL.) We wish to ask here what could be an appropriate 'fractal homology theory' in the present context. More

\footnotetext{
${ }^{168}$ See also the recent work Har4 in which $\mu(\infty)$ does not seem to play a role but whose proposed geometric and algebraic language and formalism for number theory and 'varieties' over $\mathbb{F}_{1}$ is quite appealing and should, in the long term, be connected with aspects of the present theory.
} 
specifically, in the geometric context of bounded subsets of $\mathbb{R}^{N}$ (and, more generally, of RFDs in $\mathbb{R}^{N}$ ), can one construct a suitable fractal homology theory whose dual theory would be the corresponding fractal cohomology theory (associated with the divisors of fractal zeta functions, as described above) 169 Is there an appropriate generalization of Poincaré duality and of Künneth's formula in this context? What about the more general (but at present, less geometric) context of general (and suitable) meromorphic functions?

(iii) Singularities of functions of several complex variables. Finally, we mention that as was alluded to in Remark 4.3, it would also be interesting to extend the theory of complex fractal dimensions and the associated fractal cohomology theory to (suitable) meromorphic functions of several complex variables naturally defined on complex manifolds (or, more generally on complex analytic spaces), as well as to develop an associated theory of (generalized) sheaves and sheaf cohomology (see, e.g., GunRos and Ebe for the classic theory).

Just as in the case of a single complex variable, we need not restrict ourselves to meromorphic functions defined on domains of $\mathbb{C}^{r}$, with $r \geq 1$. One is therefore naturally led to consider (nonremovable) 'singularities' of functions that go beyond the mere poles, as is already apparent in the present geometric theory of complex fractal dimensions; see, e.g., \$2.5 and \$3.6 above, along with LapRaZu10 for a first model in the case when $r=1$ and thus when suitable Riemann surfaces would be associated with the given singularities.

For more information on fractal cohomology theory and the many associated open problems, we refer the interested reader to (the latter part of) the author's forthcoming book, Lap10.

\section{Acknowledgements}

This may be a fitting place to express my deep gratitude to Erin P. J. Pearse, John A. Rock and Tony Samuel, the organizers of the International Conference and Summer School (supported by the National Science Foundation) on Fractal Geometry and Complex Dimensions held on the occasion of my sixtieth birthday in the beautiful setting of the campus of the California Polytechnic State University at San Luis Obispo, as well as to the editors of this volume of Contemporary Mathematics (the proceedings volume of the above SLO meeting), the aforementioned organizers along with Robert G. Niemeyer, for their great patience in waiting (with some anxiety, I presume) for my contribution and for their helpful suggestions for shortening the original text (which has now become the foundation for the author's forthcoming book, Lap10).

I wish to thank all of my wonderful collaborators over the past twenty five to thirty years on many aspects of the theory of complex fractal dimensions and its various extensions or related topics, including (in rough chronological order) Jacqueline Fleckinger ( FlLap and other papers), Carl Pomerance LapPo1-3, Helmut Maier LapMa1-2, Jun Kigami KiLap1-2, Michael M. H. Pang LapPa, Cheryl A. Griffith, John W. Neuberger and Robert J. Renka [LapNRG], Christina Q. He HeLap, Machiel van Frankenhuijsen [Lap-vF1-7], Ben M. Hambly HamLap,

${ }^{169}$ At least for certain self-similar geometries, there seems to be glimpses of such a theory, in which the underlying complex dimensions play a natural role. 
Erin P. J. Pearse LapPe1-3, Hung (Tim) Lu ([LapLu1-3, [LapLu-vF1-2]), John A. Rock ( LapRo, LapRoZu, LapRaRo $)$, Erik Christensen and Cristina Ivan ChrIvLap, Jacques Lévy Vehel LapLevyRo, Steffen Winter LapPeWi1-2, Nishu Lal LalLap1-2, Hafedh Herichi HerLap1-5, Rolando de Santiago and Scott A. Roby deSLapRRo, Robyn L. Miller and/or Robert Niemeyer (in papers on fractal billiards), Jonathan J. Sarhad LapSar, Goran Radunović and Darko Žubrinić LapRaZu1-10, Kate E. Ellis and Michael Mackenzie ElLapMcRo, Tim Cobler CobLap1-2 and Ryszard Nest LapNes.

I also wish to thank my many diverse and talented Ph.D. students (soon twenty of them, this year, in 2018) and postdocs, a number of whom have been mentioned among my collaborators just above.

Furthermore, I am most grateful to the two very conscientious and thorough referees of this paper for their very helpful suggestions, questions and comments.

Moreover, I am very indebted to my personal Administrative Assistant, Ms. Yu-Tzu Tsai, without whose invaluable help this paper would never have been completed (almost) on time.

Finally, I would like to gratefully acknowledge the long-term support of the U.S. National Science Foundation (NSF) over the past thirty years, since the beginning of the theory of complex dimensions and throughout much of its prehistory, under the research grants DMS-8703138, DMS-8904389, DMS-9207098, DMS-9623002, DMS-0070497, DMS-0707524 and DMS-1107750.

\section{Glossary}

$a_{k} \sim b_{k}$ as $k \rightarrow \infty$, asymptotically equivalent sequences.............13

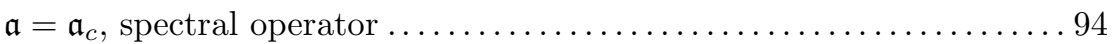

$\mathfrak{a}^{(T)}=\mathfrak{a}_{c}^{(T)}$, truncated spectral operator ................... 96

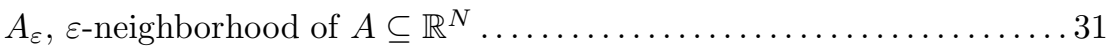

$\mathcal{A}=\mathcal{A}_{c}$, global spectral operator ........................ 97

$\mathcal{B}\left(\mathbb{H}_{c}\right)$, Banach algebra of bounded linear operators on the Hilbert space

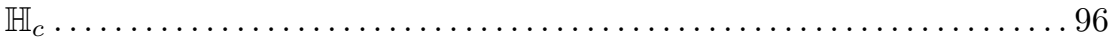

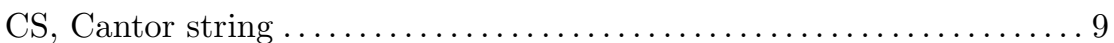

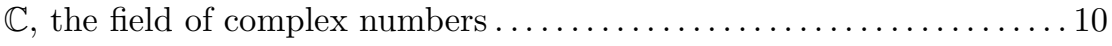

$\widetilde{\mathbb{C}}:=\mathbb{C} \cup\{\infty\}$, the Riemann sphere ....................... 95

$c \ell(A)$ or $\bar{A}$, closure of a subset $A$ of $\mathbb{C}$ or of $\mathbb{R}^{N} \ldots \ldots \ldots \ldots \ldots . \ldots 35,95$

$\underline{D}, \bar{D}$, lower, upper Minkowski dimension (of a $\operatorname{RFD}(A, \Omega)$ in $\left.\mathbb{R}^{N}\right) \ldots 31$

$D$, Minkowski dimension (of a fractal string $\mathcal{L}$ or of $A \subseteq \mathbb{R}^{N}$, or else of a

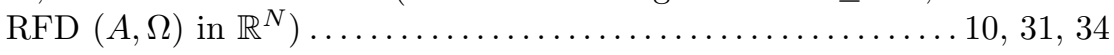

$\mathcal{D}=\mathcal{D}_{\mathcal{L}}$, the set of complex dimensions of a fractal string $\mathcal{L}$ (or of its boundary) $\ldots \ldots \ldots \ldots \ldots \ldots \ldots \ldots \ldots \ldots \ldots \ldots \ldots \ldots \ldots \ldots \ldots \ldots \ldots \ldots \ldots \ldots, 11$

$\operatorname{dim}_{\mathbb{C}} \mathcal{L}=\mathcal{D}_{\mathcal{L}}(\mathbb{C})$, the set of all complex dimensions (in $\mathbb{C}$ ) of the fractal

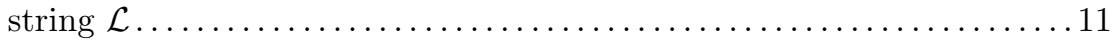

$\operatorname{dim}_{P C} \mathcal{L}=\mathcal{D}_{P C}(\mathcal{L})$, the set of principal complex dimensions of the fractal

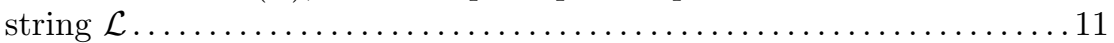


$\operatorname{dim}_{\mathbb{C}}(A, \Omega)=\mathcal{D}_{A, \Omega}(\mathbb{C})$, the set of all complex dimensions (in $\mathbb{C}$ ) of the

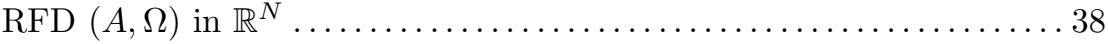
$\operatorname{dim}_{P C}(A, \Omega)=\mathcal{D}_{P C}(A, \Omega)$, the set of principal complex dimensions of

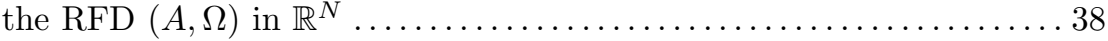

$\mathcal{D}\left(\zeta_{\mathcal{L}}\right)=\mathcal{D}\left(\zeta_{\mathcal{L}} ; U\right)$, the set of (visible) poles (in $U$ ) of the geometric zeta

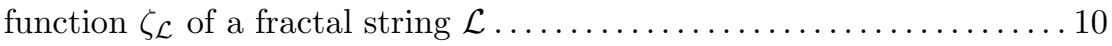

$\mathcal{D}_{\mathcal{L}}=\mathcal{D}_{\mathcal{L}}(U)$, the set of (visible) complex dimensions of a fractal string

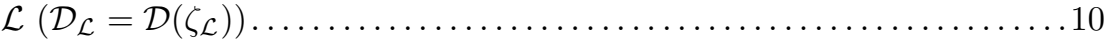

$\mathcal{D}\left(\zeta_{A, \Omega}\right)=\mathcal{D}\left(\zeta_{A, \Omega} ; U\right)$ or $\mathcal{D}\left(\widetilde{\zeta}_{A, \Omega} ; U\right)$, the set of (visible) poles (in $U$ ) of the distance or tube zeta function $\zeta_{A, \Omega}$ or $\widetilde{\zeta}_{A, \Omega}$ of an $\operatorname{RFD}(A, \Omega)$ in $\mathbb{R}^{N}$

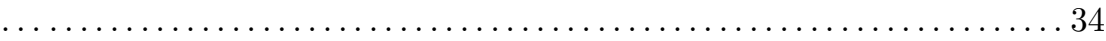
$\mathcal{D}\left(\zeta_{A}\right)=\mathcal{D}\left(\zeta_{A} ; U\right)$ or $\mathcal{D}\left(\widetilde{\zeta}_{A}\right)=\mathcal{D}\left(\widetilde{\zeta}_{A} ; U\right)$, the set of (visible) poles (in $U$ ) of the distance or tube zeta function $\zeta_{A}$ or $\widetilde{\zeta}_{A}$ of a bounded set $A$ in $\mathbb{R}^{N}$

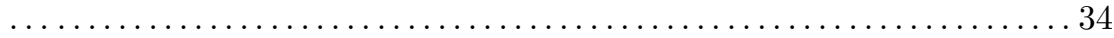
$\mathcal{D}=\mathcal{D}_{A}$ or $\mathcal{D}=\mathcal{D}_{A, \Omega}$, the set of (visible) complex dimensions of a bounded set $A$ or of an RFD in $\mathbb{R}^{N}\left(\mathcal{D}_{A}=\mathcal{D}\left(\zeta_{A}\right)=\mathcal{D}\left(\widetilde{\zeta}_{A}\right)\right.$ and $\mathcal{D}_{A, \Omega}=$

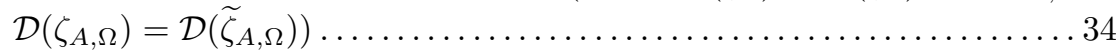
$\mathcal{D}_{\mathfrak{s}}$, the set of scaling complex dimensions (of a self-similar spray or set)

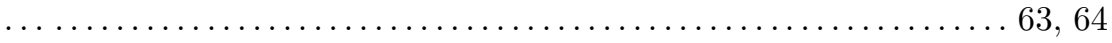
$\mathbb{D}_{\mathcal{Z}}=\mathbb{D}_{h}$, generalized Polya-Hilbert operator (GPO or GPH) associated with the divisor $\mathcal{Z}$ of a meromorphic function $h \ldots \ldots \ldots \ldots \ldots \ldots 1$ $\mathfrak{D}(f)$, divisor of a meromorphic function $f \ldots \ldots \ldots \ldots \ldots \ldots \ldots, 60$ $d(x, A)=\operatorname{dist}(x, A)=\inf \{|x-a|: a \in A\}$, Euclidean distance from $x$ to

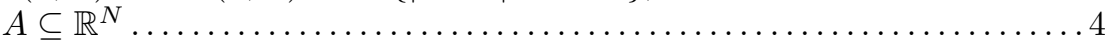

$\partial=\partial_{c}$, infinitesimal shift of the real line $\left(\partial=d / d t\right.$, acting on $\left.\mathbb{H}_{c}\right) \ldots 94$ $\partial=d / d z$, infinitesimal shift of the complex plane (acting on a suitable weighted Bergman space) $\ldots \ldots \ldots \ldots \ldots \ldots \ldots \ldots \ldots \ldots \ldots \ldots 9 . \ldots \ldots$ $\partial^{(T)}=\partial_{c}^{(T)}$, truncated infinitesimal shift (of the real line) $\ldots \ldots \ldots . .96$ $\partial \Omega$, boundary of a subset $\Omega$ of $\mathbb{R}^{N}$ (or of a fractal string) ..........33 $|E|=|E|_{N}, N$-dimensional Lebesgue measure of $E \subseteq \mathbb{R}^{N}, 10(N=1), .31$

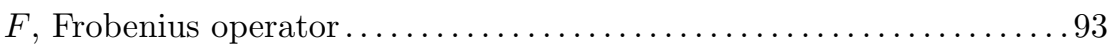
$\mathcal{F}_{\mathcal{Z}}=\mathcal{F}_{h}$, generalized Frobenius operator, associated with the divisor $\mathcal{Z}$ of a meromorphic function $h \ldots \ldots \ldots \ldots \ldots \ldots \ldots \ldots \ldots \ldots \ldots . \ldots . \ldots . \ldots 9$

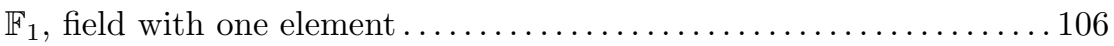

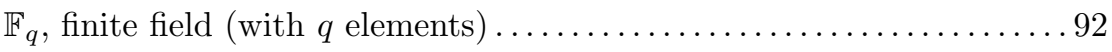

GFO, generalized Frobenius operator ....................... 99

GPO (or GPH), generalized Polya-Hilbert operator .......... 99, 101

$\Gamma(t):=\int_{0}^{+\infty} x^{t-1} e^{-x} d x$, the gamma function .............. 57, 99 $H_{\mathcal{Z}}=H_{h}$, total eigenspace of the GPO $\mathbb{D}_{\mathcal{Z}}$ associated with the divisor $\mathcal{Z}$ of a meromorphic function $h \ldots \ldots \ldots \ldots \ldots \ldots \ldots \ldots \ldots \ldots 10.105$ 
$\mathcal{H}_{\mathcal{Z}}=\mathcal{H}_{h}$, weighted Bergman space associated with the divisor $\mathcal{Z}$ of a meromorphic function $h \ldots \ldots \ldots \ldots \ldots \ldots \ldots \ldots \ldots \ldots \ldots \ldots . \ldots 9,105$ $\mathbb{H}_{c}:=L^{2}\left(\mathbb{R}, e^{-2 c t} d t\right)$, weighted Hilbert space $\ldots \ldots \ldots \ldots \ldots \ldots \ldots . \ldots 4$

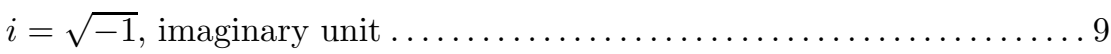
$(\mathrm{ISP})_{D}$, inverse spectral problem for the fractal strings of Minkowski di-

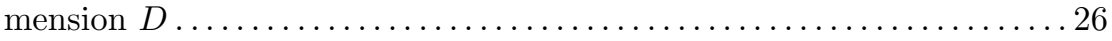
$\xi=\xi(s)$, global (or completed) Riemann zeta function............. 99 $\log _{a} x$, the logarithm of $x>0$ with base $a>0 ; y=\log _{a} x \Leftrightarrow x=a^{y} \ldots 9$ $\log x:=\log _{e} x$, the natural $\operatorname{logarithm}$ of $x ; y=\log x \Leftrightarrow x=e^{y} \ldots \ldots \ldots 9$ $\mathcal{L}=\left\{\ell_{j}\right\}_{j=1}^{\infty}$, a fractal string with lengths $\ell_{j} \ldots \ldots \ldots \ldots \ldots \ldots \ldots$

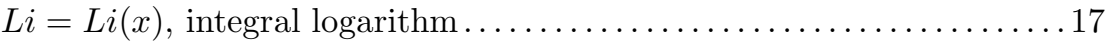
$\mathcal{M}, \mathcal{M}_{*}$ and $\mathcal{M}^{*}$, Minkowski content, lower and upper Minkowski content (of $\mathcal{L}$ or of $A \subseteq \mathbb{R}^{N}$ or else of a $\operatorname{RFD}(A, \Omega)$ in $\mathbb{R}^{N}$ ) $\ldots \ldots \ldots 12,13,32$ Moran's equation (complexified) ..........................64 $\mu(n)$, Möbius function ................................ 96 $N_{\mathcal{L}}$, geometric counting function of a fractal string $\mathcal{L} \ldots \ldots \ldots \ldots 14,15$ $N_{\nu}$, spectral (or frequency) counting function of a fractal string or drum ......................................... 14, 15, 24 $\Omega_{\varepsilon}, \varepsilon$-neighborhood of a fractal string (or of a fractal drum) $\ldots \ldots 10,31$ $\mathcal{P}$, the set of prime numbers............................. 16

$\Pi_{\mathcal{P}}$, prime number counting function $\ldots \ldots \ldots \ldots \ldots \ldots \ldots \ldots \ldots \ldots \ldots \ldots \ldots$

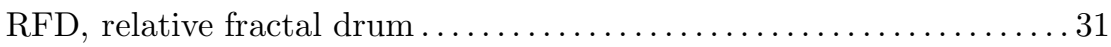

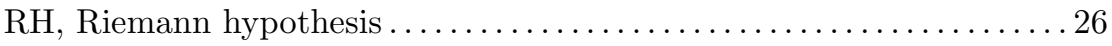

$(\mathrm{RH})_{D}$ or $\mathrm{RH}_{D}$, partial Riemann hypothesis (in dimension $\left.D\right) \ldots \ldots .27$ $\operatorname{res}(f, \omega)$, residue of the meromorphic function $f$ at $\omega \in \mathbb{C} \ldots \ldots .5,12$

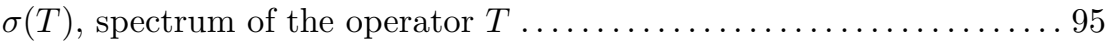
$\widetilde{\sigma}(T)$, extended spectrum of the operator $T \ldots \ldots \ldots \ldots \ldots \ldots \ldots \ldots \ldots$ $V(\varepsilon)=V_{\mathcal{L}}(\varepsilon)$ (or $V_{A, \Omega}(\varepsilon)$ or $V_{A}(\varepsilon)$ ), volume of the $\varepsilon$-neighborhood of a fractal string $\mathcal{L}$ (or of a $\operatorname{RFD}(A, \Omega)$ or of $A \subseteq \mathbb{R}^{N}$ ) $\ldots \ldots \ldots \ldots 11,31$

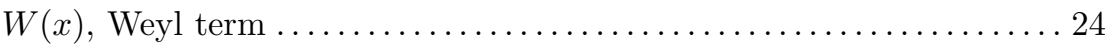

$\Xi(s)$, completed Riemann zeta function (second version) ............102

$\zeta(s)=\sum_{j=1}^{\infty} j^{-s}$, Riemann zeta function ................... 15

$\zeta_{\mathcal{L}}(s)=\sum_{j=1}^{\infty}\left(\ell_{j}\right)^{s}$, geometric zeta function of a fractal string $\mathcal{L}=\left\{\ell_{j}\right\}_{j=1}^{\infty}$

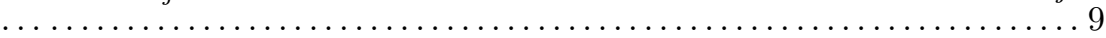

$\zeta_{\nu}=\zeta_{\nu, \mathcal{L}}$, spectral zeta function of a fractal string $\mathcal{L} \ldots \ldots \ldots \ldots \ldots 15$ $\zeta_{A, \Omega}$ or $\zeta_{A}$, distance zeta function (of a $\operatorname{RFD}(A, \Omega)$ or of a bounded set

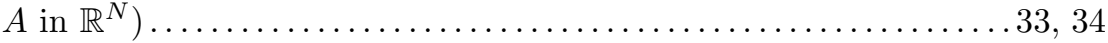
$\widetilde{\zeta}_{A, \Omega}$ or $\widetilde{\zeta}_{A}$, tube zeta function (of a $\operatorname{RFD}(A, \Omega)$ or of a bounded set $A$ in

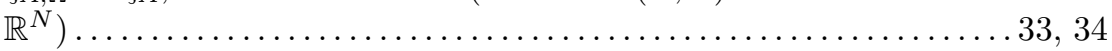




\section{References}

[AcoDur-LopGar] G. Acosta, R. G. Durán and F. López García, Korn inequality and divergence operator: Counterexamples and optimality of weighted estimates, Proc. Amer. Math. Soc. 141 (2013), 217-232.

[Art] E. Artin, Quadratische Körper im gebiet der höheren Kongruenzen, I and II. Math. Zeitschrift 19 (1924), 153-206 and 207-246.

[AtzBri] A. Atzmon and B. Brive, Surjectivity and invariant subspaces of differential operators on weighted Bergman spaces of entire functions, in: Contemporary Mathematics (A. Borichev, H. Hedenmalm and K. Zhu, eds.), vol. 404, Amer. Math. Soc., Providence, R. I., 2006, pp. $27-39$.

[Bag] B. Bagchi, The Statistical Behaviour and Universality Properties of the Riemann ZetaFunction and Other Allied Dirichlet Series, Ph.D. Thesis, Indian Statistical Institute, Calcutta, India, 1981.

[Bak] A. Baker, Transcendental Number Theory, Cambridge Univ. Press, Cambridge, 1975.

[Barl] M. T. Barlow, Diffusions on fractals, in: Lectures on Probability Theory and Statistics (P. Bernard, ed.), Ecole d'Eté de Probabilités de Saint-Flour XXV-1995, Lecture Notes in Mathematics, vol. 1690, Springer-Verlag, 1998, pp. 1-121.

[BarnDemk] M. F. Barnsley and S. Demko, Iterated function systems and the global construction of fractals, Proc. Roy. Soc. London Ser. A 399 (1985), 243-275.

[Be] A. F. Beardon, Iteration of Rational Functions, Springer-Verlag, Berlin, 1991.

[BedKS] T. Bedford, M. Keane and C. Series (eds.), Ergodic Theory, Symbolic Dynamics and Hyperbolic Spaces, Oxford Univ. Press, Oxford, 1991.

[BergGos] M. Berger and B. Gostiaux, Differential Geometry: Manifolds, Curves and Surfaces, English translation, Springer-Verlag, Berlin, 1988.

[Berr1] M. V. Berry, Distribution of modes in fractal resonators, in: Structural Stability in Physics (W. Güttinger and H. Eikemeier, eds.), Springer-Verlag, Berlin, 1979, pp. 51-53.

[Berr2] M. V. Berry, Some geometric aspects of wave motion: Wavefront dislocations, diffraction catastrophes, diffractals, in: Geometry of the Laplace Operator, Proc. Sympos. Pure Math., vol. 36, Amer. Math. Soc., Providence, R. I., 1980, pp. 13-38.

[Berr3] M. V. Berry, Riemann's zeta function: A model for quantum chaos, in: Lecture Notes in Physics, vol. 263, Springer-Verlag, Berlin, 1986, pp. 1-17.

[Berr4] M. V. Berry, The Bakerian lecture, 1987: Quantum chaology, Proc. Roy. Soc. London Ser. A 413 (1987), 183-198.

[BerrKea] M. V. Berry and J. P. Keating, The Riemann zeros and eigenvalue asymptotics, SIAM Rev. 41 (1999), 236-266.

[BesTa] A. S. Besicovitch and S. J. Taylor, On the complementary intervals of a linear closed set of zero Lesbegue measure, J. London Math. Soc. 29 (1954), 449-459.

[BirSol] M. Sh. Birman and M. Z. Solomyak, Spectral asymptotics of nonsmooth elliptic operators, I, Trans. Moscow Math. Soc. 27 (1972), 3-52; II, ibid. 28 (1973), 3-34.

[Boh] H. Bohr, Almost Periodic Functions, Chelsea, New York, 1951.

[BohCou] H. Bohr and R. Courant, Neue Anwendungen der Theorie der diophantischen Approximationen auf die Riemannsche Zetafunktion, J. Reine Angew. Math. 144 (1914), 249-274.

[BosCon1] J.-B. Bost and A. Connes, Produit eulérien et facteurs de type III, C. R. Acad. Sci. Paris Sér. I Math. 315 (1992), 279-284.

[BosCon2] J.-B. Bost and A. Connes, Hecke algebras, type III factors and phase transitions with spontaneous symmetry breaking in number theory, Selecta Math. (N.S.) 1 (1995), 411-457.

[Bou] G. Bouligand, Ensembles impropres et nombre dimensionnel, Bull. Sci. Math. (2) 52 (1928), 320-344 and 361-376.

[Br] H. Brezis, Functional Analysis, Sobolev Spaces and Partial Differential Equations, Universitext, Springer, New York, 2011. (English translation and revised and enlarged edition of H. Brezis, Analyse Fonctionnelle: Théorie et applications, Masson, Paris, 1983.)

[BroCar] J. Brossard and R. Carmona, Can one hear the dimension of a fractal?, Commun. Math. Phys. 104 (1986), 103-122.

[BruDKPW] H. Bruhn, R. Diestel, M. Kriesell, R. Pendavingh and P. Wollan, Axioms for infinite matroids, Adv. in Math. 239 (2013), 18-46.

[CaLapPe-vF] D. Carfi, M. L. Lapidus, E. P. J. Pearse and M. van Frankenhuijsen, Fractal Geometry and Dynamical Systems in Pure and Applied Mathematics, I \& II, Contemporary 
Mathematics, vols. 600 and 601, Amer. Math. Soc., Providence, R. I., 2013. (I: Fractals in Pure Mathematics, vol. 600. II: Fractals in Applied Mathematics, vol. 601.)

[Cae] A. M. Caetano, On the search for the asymptotic behaviour of the eigenvalues of the Dirichlet Laplacian for bounded irregular domains, Internat. J. Appl. Sci. Comput. 2 (1995), 261-287.

[CarlJonYoc] L. Carleson, P. W. Jones and J.-C. Yoccoz, Julia and John, Bol. Soc. Bresil Math. 25 (1994), 1-30.

[Cart] P. Cartier, A mad day's work: from Grothendieck to Connes and Kontsevich. The evolution of concepts of space and symmetry (English translation of the French original), Bull. Amer. Math. Soc. (N.S.) 38 (2001), 389-408.

[ChrIvLap] E. Christensen, C. Ivan and M. L. Lapidus, Dirac operators and spectral triples for some fractal sets built on curves, Adv. in Math. No. 1, 217 (2008), 42-78. (Also: e-print, arXiv:math.MG/0610222v2, 2007.)

[ChuOsgPomm] M. Chuaqui, B. Osgood and Ch. Pommerenke, John domains, quasidisks, and the Nehari class, J. reine angew. Math. 471 (1996), 77-114.

[CipGIS] F. Cipriani, D. Guido, T. Isola and J.-L. Sauvageot, Spectral triples for the Sierpinski gasket, J. Funct. Anal. No. 8, 266 (2014), 4809-4869.

[CobLap1] T. Cobler and M. L. Lapidus, Towards a fractal cohomology: Spectra of PolyaHilbert operators, regularized determinants and Riemann zeros, in: Exploring the Riemann Zeta Function: 190 Years from Riemann's Birth (H. Montgomery, A. Nikeghbali and M. Rassias, eds.), Springer, Basel, Berlin and New York, 2017, pp. 35-65. (Also: e-print, arXiv:1705.06222v4 [math.NT], 2017.)

[CobLap2] T. Cobler and M. L. Lapidus, Zeta functions and Weierstrass' factorization theorem via regularized determinants and infinitesimal shifts on weighted Bergman space (tentative title), in preparation, 2018

[Coh] D. L. Cohn, Measure Theory, Birkhäuser, Boston, 1980.

[Con1] A. Connes, Noncommutative Geometry, Academic Press, New York, 1994.

[Con2] A. Connes, Trace formula in noncommutative geometry and the zeros of the Riemann zeta function, Selecta Mathematica, New Series 5 (1999), 29-106.

[Conw] J. B. Conway, Functions of One Complex Variable, second edition, Graduate Texts in Mathematics, vol. 11, Springer-Verlag, New York, 1995.

[CouHil] R. Courant and D. Hilbert, Methods of Mathematical Physics, vol. I, English translation, Interscience, New York, 1953.

[CranMH] A. Crannell, S. May and L. Hilbert, Shifts of finite type and Fibonacci harps, Appl. Math. Lett. No. 2, 20 (2007), 138-141.

[DaMcCS] G. Dafni, R. J. McCann and A. Stancu (eds.), Analysis and Geometry of Metric Measure Spaces, Lecture Notes of the 50th Séminaire de Mathématiques Supérieures (SMS), (Montréal, 2011), CRM Proceedings \& Lecture Notes, vol. 56, Centre de Recherches Mathématiques (CRM), Montreal and Amer. Math. Soc., Providence, R. I., 2013.

[Dav] E. B. Davies, Two-dimensional Riemannian manifolds with fractal boundaries, J. London Math. Soc. (2) 49 (1994), 343-356.

[Del1] P. Deligne, La conjecture de Weil, I, Inst. Hautes Études Sci. Publ. Math. 43 (1974), 273-307.

[Del2] P. Deligne, La conjecture de Weil, II, Inst. Hautes Études Sci. Publ. Math. 52 (1980), 137-252.

[Del3] P. Deligne, et al. (eds.), Quantum Fields and Strings: A Course for Mathematicians, vols. 1 \& 2, Amer. Math. Soc., Providence, R. I., 1999.

[DemDenKö̈] B. Demir, A. Deniz, S. Koçak and A. E. Üreyen, Tube formulas for graph-directed fractals, Fractals, No. 3, 18 (2010), 349-361.

[DemKoÖÜ] B. Demir, Ş. Koçak, Y. Özdemir and A. E. Üreyen, Tube formulas for selfsimilar fractals with non-Steiner-like generators, in: Proc. Gökova Geometry-Topology Conf. (2012), Internat. Press, Somerville, MA, 2013, pp. 123-145. (Also: e-print, arXiv:0911.4966 [math.MG], 2009.)

[Den1] C. Deninger, Local $L$-factors of motives and regularized determinants, Invent. Math. 107 (1992), 135-150.

[Den2] C. Deninger, Lefschetz trace formulas and explicit formulas in analytic number theory, $J$. Reine Angew. Math. 441 (1993), 1-15. 
[Den3] C. Deninger, Evidence for a cohomological approach to analytic number theory, in: Proc. First European Congress of Mathematics (A. Joseph, et al., eds.), vol. I, Paris, July 1992, Birkhäuser-Verlag, Basel, 1994, pp. 491-510.

[Den4] C. Deninger, L-functions of mixed motives, Proc. Symposia Pure Math., vol. 55, Part 1, Providence, R. I., 1994, pp. 517-525.

[Den5] C. Deninger, Motivic L-functions and regularized determinants, Proc. Sympos. Pure Math., vol. 55, Part 1, Providence, R. I., 1994, pp. 707-743.

[Den6] C. Deninger, Some analogies between number theory and dynamical systems on foliated spaces, in: Proc. Internat. Congress Math., Berlin, 1998 (G. Fischer and U. Rehmann, eds.), vol. I, pp. 163-186. Documenta Math. J. DMV (Extra Volume ICM 98), 1998.

[DenKoÖ̈] A. Deniz, Ş. Koçak, Y. Özdemir and A. E. Üreyen, Tube volumes via functional equations, J. Geom. 105 (2014), 1-10.

[DenKoÖRÜ] A. Deniz, Ş. Koçak, Y. Özdemir, A. Ratiu and A. E. Üreyen, On the Minkowski measurability of self-similar fractals in $\mathbb{R}^{d}$, Turkish J. Math. 37 (2013), 830-846.

[DenSchr] C. Deninger and M. Schröter, A distributional theoretic proof of Guinand's functional equation for Cramér's $V$-function and generalizations, J. London Math. Soc. 52 (1995), 4860.

[dV1] C.-J. de la Vallée Poussin, Recherches analytiques sur la théorie des nombres; Première partie: La fonction $\zeta(s)$ de Riemann et les nombres premiers en général, Ann. Soc. Sci. Bruxelles Sér. I 20 (1896), 183-256.

[dV2] C.-J. de la Vallée Poussin, Sur la fonction $\zeta(s)$ de Riemann et le nombre des nombres premiers inférieurs à une limite donnée, Mém. Couronnés et Autres Mém. Publ. Acad. Roy. Sci., des Lettres, Beaux-Arts Belg. 59 (1899-1900).

[DerGrVo] G. Derfel, P. Grabner and F. Vogl, The zeta function of the Laplacian on certain fractals, Trans. Amer. Math. Soc. 360 (2008), 881-897.

[deSLapRRo] R. de Santiago, M. L. Lapidus, S. A. Roby and J. A. Rock, Multifractal analysis via scaling zeta functions and recursive structure of lattice strings, in: Fractal Geometry and Dynamical Systems in Pure and Applied Mathematics I: Fractals in Pure Mathematics (D. Carfi, M. L. Lapidus, E. P. J. Pearse and M. van Frankenhuijsen, eds.), Contemporary Mathematics, vol. 600, Amer. Math. Soc., Providence, R. I., 2013, pp. 205-238. (dx.doi.org/10.1090/conm/600/11930.) (Also, e-print, arXiv:1207.6680v3 [math-ph], 2013; IHES preprint, IHES/M/12/19, 2013.)

[DieRuzSchu] L. Diening, M. Ružička and K. Schumacher, A decomposition technique for John domains, Ann. Acad. Sci. Fenn. Math. 35 (2010), 87-114.

[Dieu1] J. Dieudonné, On the history of the Weil conjectures, Math. Intelligencer 10 (1975), 7-21.

[Dieu2] J. Dieudonné, A History of Algebraic and Differential Topology: 1900-1960, Birkhäuser, Boston, 1989.

[DubSep] E. Dubon and J. M. Sepulcre, On the complex dimensions of nonlattice fractal strings in connection with Dirichlet polynomials, J. Experimental Math. No. 1, 23 (2014), 13-24.

[DunSch] N. Dunford and J. T. Schwartz, Linear Operators, Parts I-III, Wiley Classics Library, John Wiley \& Sons, Hoboken, 1971 and 1988. (Part I: General Theory. Part II: Spectral Theory. Part III: Spectral Operators.)

[Dur-LopGar] R. G. Durán and F. López García, Korn inequality and divergence problems on domains with an external cusp, Ann. Acad. Sci. Fenn. Math. No. 2, 35 (2010), 421-438.

[DureSchu] P. Duren and A. Schuster, Bergman Spaces, Mathematical Surveys and Monographs, vol. 100, Amer. Math. Soc., Providence, R. I., 2004.

[Ebe] W. Ebeling, Functions of Several Complex Variables and Their Singularities, Graduate Studies in Mathematics, vol. 83, Amer. Math. Soc., Providence, R. I., 2007.

[EdmEv] D. E. Edmunds and W. D. Evans, Fractals, trees and the Neumann Laplacian, Math. Annalen 296 (1993), 493-527.

[Edw] H. M. Edwards, Riemann's Zeta Function, Academic Press, New York, 1974. (Reprinted by Dover Publ., Mineola, 2001.)

[ElLapMcRo] K. E. Ellis, M. L. Lapidus, M. C. Mackenzie and J. A. Rock, Partition zeta functions, multifractal spectra, and tapestries of complex dimensions, in: Benoit Mandelbrot: A Life in Many Dimensions, The Mandelbrot Memorial Volume (M. Frame and N. Cohen, eds.), World Scientific, Singapore, 2015, pp. 267-312. (Also: e-print, arXiv:1007.1467v2 [math-ph], 2011; IHES preprint, IHES/M/12/15, 2012.) 
[Es1] D. Essouabri, Singularités des séries de Dirichlet associées à des polynômes de plusieurs variables et applications en théorie analytique des nombres, Ann. Inst. Fourier (Grenoble) 47 (1996), 429-484.

[Es2] D. Essouabri, Zeta functions associated to Pascal's triangle mod p, Japan J. Math. (New Series) 31 (2005), 157-174.

[EsLi1] D. Essouabri and B. Lichtin, Zeta functions of discrete self-similar sets, Adv. in Math. 232 (2013), 142-187.

[EsLi2] D. Essouabri and B. Lichtin, $k$-point configurations of discrete self-similar sets, in: Fractal Geometry and Dynamical Systems in Pure and Applied Mathematics I:Fractals in Pure Mathematics (D. Carfi, M. L. Lapidus and M. van Frankenhuijsen, eds.), Contemporary Mathematics, vol. 600, Amer. Math. Soc, Providence, R. I., 2013, pp. 21-50.

[Fa1] K. J. Falconer, Fractal Geometry: Mathematical Foundations and Applications, third edition, John Wiley \& Sons, Chichester, 2014. (First and second editions: 1990 and 2003.)

[Fa2] K. J. Falconer, On the Minkowski measurability of fractals, Proc. Amer. Math. Soc. 123 (1995), 1115-1124.

[Fed1] H. Federer, Curvature measures, Trans. Amer. Math. Soc. 93 (1959), 418-491.

[Fed2] H. Federer, Geometric Measure Theory, Springer-Verlag, New York, 1969.

[Fel] W. Feller, An Introduction to Probability Theory and Its Applications, vol. II, John Wiley \& Sons, New York, 1966.

[FlLap] J. Fleckinger and M. L. Lapidus, Remainder estimates for the asymptotics of elliptic eigenvalue problems with indefinite weights, Archiv. Rat. Mech. Anal. No. 4, 98 (1987), 329-356.

[FlVa] J. Fleckinger and D. Vassiliev, An example of a two-term asymptotics for the "counting function" of a fractal drum, Trans. Amer. Math. Soc. 337 (1993), 99-116.

[Fo] G. B. Folland, Real Analysis: Modern Techniques and Their Applications, second edition, John Wiley \& Sons, Boston, 1999.

[Fr] M. Frantz, Lacunarity, Minkowski content, and self-similar sets in $\mathbb{R}$, in: Fractal Geometry and Applications: A Jubilee of Benoit Mandelbrot (M. L. Lapidus and M. van Frankenhuijsen, eds.), Proc. Sympos. Pure Math. vol. 72, Part 1, Amer. Math. Soc., Providence, R. I., 2004, pp. $77-91$.

[FreiKom] U. Freiberg and S. Kombrink, Minkowski content and local Minkowski content for a class of self-conformal sets, Geom. Dedicata 159 (2012), 307-325.

[Fred] J. Fredholm, Sur une classe d'équations fonctionnelles, Acta Math. 27 (1903), 365-390.

[FukShi] M. Fukushima and T. Shima, On a spectral analysis for the Sierpinski gasket, Potential Analysis 1 (1992), 1-35.

[GarSteu] R. Garunkštis and J. Steuding, On the roots of the equation $\zeta(s)=\alpha$, Abh. Math. Seminar Univ. Hamburg 84 (2014), 1-15. (Also: e-print, arXiv:1011.5339v2 [math.NT], 2014.)

[Gat] D. Gatzouras, Lacunarity of self-similar and stochastically self-similar sets, Trans. Amer. Math. Soc. 352 (2000), 1953-1983.

[GelSh] I. M. Gelfand and G. E. Shilov, Generalized Functions, vols. I-III, Academic Press, new edition, 1986.

[Ger] J. Gerling, Untersuchungen zur Theorie von Weyl-Berry-Lapidus, Graduate thesis (diplomarbeit), Dept. of Physics, Universität Osnabrück, Germany, 1992.

[GerScm1] J. Gerling and H.-J. Schmidt, Self-similar drums and generalized Weierstrass functions, Physica A 191 (1992), 536-539.

[GerScm2] J. Gerling and H.-J. Schmidt, Three-term asymptotics of the spectrum of self-similar fractal drums, J. Math. Sci. Univ. Tokyo 6 (1999), 101-126.

[Gi] P. B. Gilkey, Invariance Theory, the Heat Equation, and the Atiyah-Singer Index Theorem, second edition, Publish or Perish, Wilmington, 1984. (New revised and enlarged edition in Studies in Advanced Mathematics, CRC Press, Boca Raton, 1995.)

[Go] J. A. Goldstein, Semigroups of Linear Operators and Applications, Oxford Science Publications, Oxford Mathematical Monographs, Oxford Univ. Press, Oxford and New York, 1985.

[GohKre] I. C. Goh'berg and M. G. Krein, Introduction to the Theory of Linear Nonselfadjoint Operators, Translations of Mathematical Monographs, Amer. Math. Soc., Providence, R. I., 1969. 
[Gra] A. Gray, Tubes, second and revised edition (of the 1990 edn.), Progress in Math., vol. 221, Birkhäuser, Boston, 2004.

[Gro1] A. Grothendieck, The cohomology theory of abstract algebraic varieties, in: Proc. Internat. Congress Math. (Edinburgh, 1958), 1960, pp. 103-118.

[Gro2] A. Grothendieck, Formule de Lefschetz et rationalité des fonctions 1, Séminaire N. Bourbaki, Exp. 279, 1964-1966, pp. 41-55.

[Gro3] A. Grothendieck, Standard conjectures on algebraic cycles, in: Algebraic Geometry (Internat. Colloq., Tata Inst. Fund. Res., Bombay, 1968), 1969, pp. 193-199.

[Gro4] A. Grothendieck, Eléments de Géométrie Algébrique, Inst. Hautes Études Sci. Publ. Math., vols. 4, 11, 17, 20, 24, 28 and 32. (With the collaboration of J. Dieudonné.) [Reprinted in 1999 by the Institut des Hautes Études Scientifiques (IHES), Bures-sur-Yvette, France.]

[GunRos] C. R. Gunning and H. Rossi, Analytic Functions of Several Complex Variables, AMS Chelsea Publishing, Amer. Math. Soc., Providence, R. I., 2015. (Reprinted from the 1965 edition.)

[Had1] J. Hadamard, Étude sur les propriétés des fonctions entières et en particulier d'une fonction considérée par Riemann, J. Math. Pures Appl. (4) 9 (1893), 171-215. (Reprinted in Had3. pp. 103-147].)

[Had2] J. Hadamard, Sur la distribution des zéros de la fonction $\zeta(s)$ et ses conséquences arithmétiques, Bull. Soc. Math. France 24 (1896), 199-220. (Reprinted in Had3 pp. 189210].)

[Had3] J. Hadamard, Oeuvres de Jacques Hadamard, Tome I, Editions du Centre National de la Recherche Scientifique, Paris, 1968.

[HajKosTu1] P. Hajlasz, P. Koskela and H. Tuominen, Sobolev embeddings, extensions and measure density condition, J. Funct. Anal. No. 5, 254 (2008), 1217-1234.

[HajKosTu2] P. Hajlasz, P. Koskela and H. Tuominen, Measure density and extendability of functions, Rev. Mat. Iberomaricana No. 2, 24 (2008), 645-669.

[Ham1] B. M. Hambly, Brownian motion on a random recursive Sierpinski gasket, Ann. Probab. 25 (1997), 1059-1102.

[Ham2] B. M. Hambly, On the asymptotics of the eigenvalue counting function for random recursive Sierpinski gaskets, Probab. Theory Related Fields 117 (2000), 221-247.

[HamLap] B. M. Hambly and M. L. Lapidus, Random fractal strings: their zeta functions, complex dimensions and spectral asymptotics, Trans. Amer. Math. Soc. No. 1, 358 (2006), 285-314.

[Har1] S. Haran, Riesz potentials and explicit sums in arithmetic, Invent. Math. 101 (1990), 696-703.

[Har2] S. Haran, The Mysteries of the Real Prime, London Mathematical Society Monographs, New Series, vol. 25, Oxford Univ. Press, Oxford, 2001.

[Har3] S. Haran, On Riemann's zeta function, in: Dynamical, Spectral, and Arithmetic Zeta Functions (M. L. Lapidus and M. van Frankenhuysen), Contemporary Mathematics, vol. 290, Amer. Math. Soc., Providence, R. I., 2001, pp. 93-112.

[Har4] S. Haran, New Foundations for Geometry: Two Non-Additive Languages for Arithmetical Geometry, Memoirs Amer. Math. Soc. No. 1166, 246 (2017), 1-202.

[Hart] R. Hartshorne, Algebraic Geometry, Graduate Texts in Mathematics, Springer-Verlag, New York, 1977.

[HarvPol] R. Harvey and J. Polking, Removable singularities of solutions of linear partial differential equations, Acta Math. 125 (1970), 39-56.

[Has] H. Hasse, Abstrakte Begründung der komplexen Multiplikation und Riemannsche Vermutung in Funktionenkörpern, Anh. Math. Sem. Hamburg 10 (1934), 325-348.

[Hat] M. Hata, On some properties of set-dynamical systems, Proc. Japan Academy Ser. A 61 (1985), 99-102.

[HeLap] C. Q. He and M. L. Lapidus, Generalized Minkowski Content, Spectrum of Fractal Drums, Fractal Strings and the Riemann Zeta-Function, Memoirs Amer. Math. Soc. No. 608, 127 (1997), 1-97.

[HedKonZhu] H. Hedenmalm, B. Korenblum and K. Zhu, Theory of Bergman Spaces, Graduate Texts in Mathematics, vol. 199, Springer, New York, 2000.

[HerLap1] H. Herichi and M. L. Lapidus, Quantized Number Theory, Fractal Strings and the Riemann Hypothesis: From Spectral Operators to Phase Transitions and Universality, research monograph, World Scientific, Singapore and London, 2018, in press, approx. 380 pages. ISBN: 978-981-3230-79-8. 
[HerLap2] H. Herichi and M. L. Lapidus, Riemann zeros and phase transitions via the spectral operator on fractal strings, J. Phys. A: Math. Theor. 45 (2012), 374005, 23pp. (Special issue in honor of Prof. Stewart Dowker's 75th birthday.) (Also: e-print, arXiv:1203.4828v2 [math-ph], 2012; IHES preprint, IHES/M/12/09, 2012.)

[HerLap3] H. Herichi and M. L. Lapidus, Fractal complex dimensions, Riemann hypothesis and invertibility of the spectral operator, in: Fractal Geometry and Dynamical Systems in Pure and Applied Mathematics I: Fractals in Pure Mathematics (D. Carfi, M. L. Lapidus, E. P. J. Pearse and M. van Frankenhuijsen, eds.), Contemporary Mathematics, vol. 600, Amer. Math. Soc., Providence, R. I., 2013, pp. 51-89. (dx.doi.org/10.1090/conm/600/11948.) (Also: e-print, arXiv:1210.0882v3 [math.FA], 2013; IHES preprint, IHES/M/12/25, 2012.)

[HerLap4] H. Herichi and M. L. Lapidus, Truncated infinitesimal shifts, spectral operators and quantized universality of the Riemann zeta function, Annales de la Faculté des Sciences de Toulouse, No. 3, 23 (2014), 621-664. (Special issue in honor of Christophe Soulé.) (Also: e-print, arXiv:1305.3933v1 [math.NT], 2013; IHES preprint, IHES/M/13/12, 2013.)

[HerLap5] H. Herichi and M. L. Lapidus, Quantized Riemann zeta function: Its operator-valued Dirichlet series, Euler product and analytic continuation, in preparation, 2017.

[HilPh] E. Hille and R. S. Phillips, Functional Analysis and Semi-groups, Amer. Math. Soc. Colloq. Publ., vol. XXXI, revised edition, Amer. Math. Soc., Providence, R. I., 1957.

[Ho1] L. Hörmander, The spectral function of an elliptic operator, Acta Math. 121 (1968), 193218.

[Ho2] L. Hörmander, The Analysis of Linear Partial Differential Operators, vol. I, Distribution Theory and Fourier Analysis, second edition (of the 1983 edn.), Springer-Verlag, Berlin, 1990.

[Ho3] L. Hörmander, The Analysis of Linear Partial Differential Operators, vols. II-IV, SpringerVerlag, Berlin, 1983 \& 1985.

[HugLasWeil] D. Hug, G. Last and W. Weil, A local Steiner-type formula for general closed sets and applications, Math. Zeitschrift 246 (2004), 237-272.

[Hut] J. Hutchinson, Fractals and self-similarity, Indiana Univ. J. Math. 30 (1981), 713-747.

[Ing] A. E. Ingham, The Distribution of Prime Numbers, second edition (reprinted from the 1932 edn.), Cambridge Univ. Press, Cambridge, 1992.

[Ivi] A. Ivic, The Riemann Zeta-Function: The Theory of the Riemann Zeta-Function with Applications, John Wiley \& Sons, New York, 1985.

[Ivr1] V. Ja. Ivrii, Second term of the spectral asymptotic expansion of the Laplace-Beltrami operator on manifolds with boundary, Functional Anal. Appl. 14 (1980), 98-106.

[Ivr2] V. Ja. Ivrii, Precise Spectral Asymptotics for Ellpitic Operators Acting in Fiberings over Manifolds with Boundary, Lecture Notes in Math., vol. 1100, Springer-Verlag, New York, 1984.

[Ivr3] V. Ja. Ivrii, Microlocal Analysis and Precise Spectral Asymtotics, Springer-Verlag, Berlin, 1998.

[JoLap] G. W. Johnson and M. L. Lapidus, The Feynman Integral and Feynman's Operational Calculus, Oxford Science Publications, Oxford Mathematical Monographs, Oxford Univ. Press, Oxford and New York, 2000. (Corrected printing and paperback edition, 2002.)

[JoLapNie] G. W. Johnson, M. L. Lapidus and L. Nielsen, Feynman's Operational Calculus and Beyond: Noncommutativity and Time-Ordering, Oxford Science Publications, Oxford Mathematical Monographs, Oxford Univ. Press, Oxford and New York, 2015.

[John] F. John, Rotation and strain, Commun. Pure Appl. Math. 14 (1961), 391-414.

[Jon] P. W. Jones, Quasiconformal mappings and extendability of functions in Sobolev spaces, Acta. Math. 147 (1981), 1-61.

[Kac] M. Kac, Can one hear the shape of a drum?, Amer. Math. Monthly (Slaught Memorial Papers, No. 11) (4) $\mathbf{7 3}$ (1966), 1-23.

[Kah] B. Kahn, Motifs, in: Leçons de mathématiques d'aujourd'hui, vol. 3 (É. Charpentier and N. Nikolski, eds.), Cassini, Paris, 2007 (in French). [Motives, in: Lessons of Today's Mathematics.]

[KarVo] A. A. Karatsuba and S. M. Voronin, The Riemann Zeta-Function, De Gruyter, Expositions in Mathematics, Walter de Gruyter, Berlin, 1992.

[Kat] T. Kato, Perturbation Theory for Linear Operators, Springer-Verlag, New York, 1995.

[Katz] N. Katz, An overview of Deligne's proof of the Riemann hypothesis for varieties over finite fields, in: Proc. Symposia Pure Math., vol. 28, Amer. Math. Soc., Providence, R. I., 1976, pp. 275-305. 
[Katzn] Y. Katznelson, An Introduction to Harmonic Analysis, third edition, Cambridge Mathematical Library, Cambridge Univ. Press, Cambridge, 2009.

[KeKom] M. Kesseböhmer and S. Kombrink, Fractal curvature measures and Minkowski content for self-conformal subsets of the real line, Adv. in Math. 230 (2012), 2474-2512.

[Ki] J. Kigami, Analysis on Fractals, Cambridge Univ. Press, Cambridge, 2001.

[KiLap1] J. Kigami and M. L. Lapidus, Weyl's problem for the spectral distribution of Laplacians on p.c.f self-similar fractals, Commun. Math. Phys. 158 (1993), 93-125.

[KiLap2] J. Kigami and M. L. Lapidus, Self-similarity of volume measures for Laplacians on p.c.f self-similar fractals, Commun. Math. Phys. 217 (2001), 165-180.

[KlRot] D. A. Klain and G.-C. Rota, Introduction to Geometric Probability, Accademia Nazionale dei Lincei, Cambridge Univ. Press, Cambridge, 1999.

[KoRati] S. Koçak and A. V. Ratiu, Inner tube formulas for polytopes, Proc. Amer. Math. Soc. No. 3, 140 (2012), 999-1010. (Also: e-print, arXiv:1008.2040v1 [math.MG], 2010.)

[Kom] S. Kombrink, A survey on Minkowski measurability of self-similar sets and self-conformal fractals in $\mathbb{R}^{d}$, survey article, in: Fractal Geometry and Dynamical Systems in Pure and Applied Mathematics I: Fractals in Pure Mathematics (D. Carfi, M. L. Lapidus, E. P. J. Pearse and M. van Frankenhuijsen, eds.), Contemporary Mathematics, vol. 600, Amer. Math. Soc., Providence, R. I., 2013, pp. 135-159. (dx.doi.org/10.1090/conm/600/11931.)

[KomPeWi] S. Kombrink, E. P. J. Pearse and S. Winter, Lattice-type self-similar sets with pluriphase generators fail to be Minkowski measurable, Math. Zeitschrift, No. 3, 283 (2016), 1049-1070. (Also: e-print, arXiv:1501.03764v1 [math.PR], 2015.)

[Kor] J. Korevaar, Tauberian Theory: A Century of Developments, Springer-Verlag, Heidelberg, 2004.

[Lag] J. C. Lagarias, Number theory zeta functions and dynamical zeta functions, in: Spectral Problems in Geometry and Arithmetic (T. Branson, ed.), Contemporary Mathematics, vol. 237, Amer. Math. Soc., Providence, R. I., 1999, pp. 45-86.

[LalLap1] N. Lal and M. L. Lapidus, Hyperfunctions and spectral zeta functions of Laplacians on self-similar fractals, J. Phys. A: Math. Theor. 45 (2012), 365205, 14pp. (Also: e-print, arXiv:1202.4126v2 [math-ph], 2012; IHES preprint, IHES/M/12/14, 2012.)

[LalLap2] N. Lal and M. L. Lapidus, The decimation method for Laplacians on fractals: Spectra and complex dynamics, in: Fractal Geometry and Dynamical Systems in Pure and Applied Mathematics II: Fractals in Applied Mathematics (D. Carfi, M. L. Lapidus, E. P. J. Pearse and M. van Frankenhuijsen, eds.), Contemporary Mathematics, vol. 601, Amer. Math. Soc., Providence, R. I., 2013, pp. 227-249. (dx.doi.org/10.1090/conm/601/11959.) (Also: e-print, arXiv: 1302.4007v2 [math-ph], 2014; IHES preprint, IHES/M/12/31, 2012.)

[Lall1] S. P. Lalley, Packing and covering functions of some self-similar fractals, Indiana Univ. Math. J. 37 (1988), 699-709.

[Lall2] S. P. Lalley, Renewal theorems in symbolic dynamics, with applications to geodesic flows, noneuclidean tessellations and their fractal limits, Acta Math. 163 (1989), 1-55.

[Lall3] S. P. Lalley, Probabilistic counting methods in certain counting problems of ergodic theory, in BedKS pp. 223-258].

[Lap1] M. L. Lapidus, Fractal drum, inverse spectral problems for elliptic operators and a partial resolution of the Weyl-Berry conjecture, Trans. Amer. Math. Soc. 325 (1991), 465-529.

[Lap2] M. L. Lapidus, Spectral and fractal geometry: From the Weyl-Berry conjecture for the vibrations of fractal drums to the Riemann zeta-function, in: Differential Equations and Mathematical Physics (C. Bennewitz, ed.), Proc. Fourth UAB Internat. Conf. (Birmingham, March 1990), Academic Press, New York, 1992, pp. 151-182.

[Lap3] M. L. Lapidus,Vibrations of fractal drums, the Riemann hypothesis, waves in fractal media, and the Weyl-Berry conjecture, in: Ordinary and Partial Differential Equations (B. D. Sleeman and R. J. Jarvis, eds.), vol. IV, Proc. Twelfth Internat. Conf. (Dundee, Scotland, UK, June 1992), Pitman Research Notes in Math. Series, vol. 289, Longman Scientific and Technical, London, 1993, pp. 126-209.

[Lap4] M. L. Lapidus, Fractals and vibrations: Can you hear the shape of a fractal drum?, Fractals, No. 4, 3 (1995), 725-736. (Special issue in honor of Benoit B. Mandelbrot's 70th birthday.)

[Lap5] M. L. Lapidus, Analysis on fractals, Laplacians on self-similar sets, noncommutative geometry and spectral dimensions, Topological Methods in Nonlinear Analysis 4 (1994), 137195. (Special issue dedicated to Jean Leray.) 
[Lap6] M. L. Lapidus, Towards a noncommutative fractal geometry? Laplacians and volume measures on fractals, in: Harmonic Analysis and Nonlinear Differential Equations (A Volume in Honor of Victor L. Shapiro), Contemporary Mathematics, vol. 208, Amer. Math. Soc., Providence, R. I., 1997, pp. 211-252.

[Lap7] M. L. Lapidus, In Search of the Riemann Zeros: Strings, Fractal Membranes and Noncommutative Spacetimes, research monograph, Amer. Math. Soc., Providence, R. I., 2008.

[Lap8] M. L. Lapidus, Towards quantized number theory: Spectral operators and an asymmetric criterion for the Riemann hypothesis, Philos. Trans. Royal Soc. Ser. A, No. 2047, 373 (2015), 24pp. DOI: 10.1098/rsta.2014.0240. (Special issue titled "Geometric Concepts in the Foundations of Physics".) (Also: e-print, arXiv:1501.05362v2 [math-ph], 2015; IHES preprint, IHES/M/15/12, 2015.))

[Lap9] M. L. Lapidus, The sound of fractals strings and the Riemann hypothesis, in: Analytic Number Theory: In Honor of Helmut Maier's 60th Birthday (C. B. Pomerance and T. Rassias, eds.), Springer Internat. Publ. Switzerland, Cham, 2015, pp. 201-252. (Also: e-print, arXiv:1505.01548v1 [math-ph], 2015; IHES preprint, IHES/M/15/11, 2015.)

[Lap10] M. L. Lapidus, From Complex Fractal Dimensions and Quantized Number Theory To Fractal Cohomology: A Tale of Oscillations, Unreality and Fractality, book in preparation, 2018, approx. 400 pages.

[LapLevyRo] M. L. Lapidus, J. Lévy Vehel and J. A. Rock, Fractal strings and multifractal zeta functions, Lett. Math. Phys. No. 1, 88 (2009), 101-129. (Also: e-print, arXiv:math-ph/0610015v3, 2009; Springer Open Access: DOI: 10.1007/s11005-009-0302-y.)

[LapLu1] M. L. Lapidus and H. Lu, Nonarchimedean Cantor set and string, J. Fixed Point Theory and Appl. 3 (2008), 181-190. (Special issue dedicated to the Jubilee of Vladimir I. Arnold, vol. I.)

[LapLu2] M. L. Lapidus and H. Lu, Self-similar $p$-adic fractal strings and their complex dimensions, $p$-adic Numbers, Ultrametric Analysis and Applications (Springer \& Russian Academy of Sciences, Moscow), No. 2, 1 (2009), 167-180. (Also: IHES preprint, IHES/M/08/42, 2008.)

[LapLu3] M. L. Lapidus and H. Lu, The geometry of $p$-adic fractal strings: A comparative survey, in: Advances in Non-Archimedean Analysis, Proc. 11th Internat. Conference on $p$-Adic Functional Analysis (Clermont-Ferrand, France, July 2010), J. Araujo, B. Diarra and A. Escassut, eds., Contemporary Mathematics, vol. 551, Amer. Math. Soc., Providence, R. I., 2011, pp. 163-206. (Also: e-print, arXiv:1105.2966v1 [math.MG], 2011.)

[LapLu-vF1] M. L. Lapidus, H. Lu and M. van Frankenhuijsen, Minkowski measurability and exact fractal tube formulas for $p$-adic self-similar strings, in: Fractal Geometry and Dynamical Systems in Pure and Applied Mathematics I: Fractals in Pure Mathematics (D. Carfi, M. L. Lapidus, E. P. J. Pearse and M. van Frankenhuijsen, eds.), Contemporary Mathematics, vol. 600, Amer. Math. Soc., Providence, R. I., 2013, pp. 161-184. (dx.doi.org/10.1090/conm/600/11949.) (Also: e-print, arXiv:1209.6440v1 [math.MG], 2012; IHES preprint, IHES/M/12/23, 2012.)

[LapLu-vF2] M. L. Lapidus, H. Lu and M. van Frankenhuijsen, Minkowski dimension and explicit tube formulas for $p$-adic fractal strings, Fractal and Fractional, 2018. (Also: e-print, arXiv: 1603.09409v2 [math-ph], 2018.)

[LapMa1] M. L. Lapidus and H. Maier, Hypothèse de Riemann, cordes fractales vibrantes et conjecture de Weyl-Berry modifiée, C. R. Acad. Sci. Paris Sér. I Math. 313 (1991), 19-24.

[LapMa2] M. L. Lapidus and H. Maier, The Riemann hypothesis and inverse spectral problems for fractal strings, J. London Math. Soc. (2) 52 (1995), 15-34.

[LapNRG] M. L. Lapidus, J. W. Neuberger, R. J. Renka and C. A. Griffith, Snowflake harmonics and computer graphics: Numerical computation of spectra on fractal domains, Internat. J. Bifurcation \& Chaos 6 (1996), 1185-1210.

[LapNes] M. L. Lapidus and R. Nest, Fractal membranes as the second quantization of fractal strings, in preparation, 2018.

[LapPa] M. L. Lapidus and M. M. H. Pang, Eigenfunctions of the Koch snowflake drum, Commun. Math. Phys. 172 (1995), 359-376.

[LapPe1] M. L. Lapidus and E. P. J. Pearse, A tube formula for the Koch snowflake curve, with applications to complex dimensions, J. London Math. Soc. No. 2.74 (2006), 397-414. (Also: e-print, arXiv:math-ph/0412029v2, 2005.)

[LapPe2] M. L. Lapidus and E. P. J. Pearse, Tube formulas for self-similar fractals, in: Analysis on Graphs and its Applications (P. Exner, et al., eds.), Proc. Sympos. Pure Math., vol. 77, Amer. 
Math. Soc., Providence, R. I., 2008, pp. 211-230. (Also: e-print, arXiv:math.DS/0711.0173, 2007; IHES preprint, IHES/M/08/28, 2008.)

[LapPe3] M. L. Lapidus and E. P. J Pearse, Tube Formulas and complex dimensions of selfsimilar tilings, Acta Applicandae Mathematicae No. 1, 112 (2010), 91-137. (Also: e-print, arXiv:math.DS/0605527v5, 2010; Springer Open Access: DOI: 10.1007/S10440-010-9562-x.)

[LapPeWi1] M. L. Lapidus, E. P. J Pearse and S. Winter, Pointwise tube formulas for fractal sprays and self-similar tilings with arbitrary generators, Adv. in Math. 227 (2011), 13491398. (Also: e-print, arXiv:1006.3807v3 [math.MG], 2011.)

[LapPeWi2] M. L. Lapidus, E. P. J. Pearse and S. Winter, Minkowski measurability results for self-similar tilings and fractals with monophase generators, in: Fractal Geometry and Dynamical Systems in Pure and Applied Mathematics I: Fractals in Pure Mathematics (D. Carfi, M. L. Lapidus, E. P. J. Pearse and M. van Frankenhuijsen, eds.), Contemporary Mathematics, vol. 600, Amer. Math. Soc., Providence, R. I., 2013, pp. 185-203. (dx.doi.org/10.1090/conm/600/11951.) (Also: e-print, arXiv:1104.1641v3 [math.MG], 2012; IHES preprint, IHES/M/12/33, 2012.)

[LapPo1] M. L. Lapidus and C. Pomerance, Fonction zêta de Riemann et conjecture de WeylBerry pour les tambours fractals, C. R. Acad. Sci. Paris Sér. I Math. 310 (1990), 343-348.

[LapPo2] M. L. Lapidus and C. Pomerance, The Riemann zeta-function and the one-dimensional Weyl-Berry conjecture for fractal drums, Proc. London Math. Soc. (3) No. 1, 66 (1993), 41-69.

[LapPo3] M. L. Lapidus and C. Pomerance, Counterexamples to the modified Weyl-Berry conjecture on fractal drums, Math. Proc. Cambridge Philos. Soc. 119 (1996), 167-178.

[LapRaRo] M. L. Lapidus, G. Radunović and J. A. Rock, An Invitation to Fractal Geometry: Dimension Theory, Zeta Functions and Applications I \& II, books in preparation, 2018. (Two-volume set for publication in the book series Student Mathematical Library, Amer. Math. Soc., Providence, R. I.)

[LapRaZu1] M. L. Lapidus, G. Radunović and D. Žubrinić, Fractal Zeta Functions and Fractal Drums: Higher-Dimensional Theory of Complex Dimensions, Springer Monographs in Mathematics, Springer, New York, 2017, 685 pages. (ISBN: 978-3-319-44706-3.)

[LapRaZu2] M. L. Lapidus, G. Radunović and D. Žubrinić, Distance and tube zeta functions of fractals and arbitrary compact sets, Adv. in Math. 307 (2017), 1215-1267. (dx.doi.org/10.1016/j.aim2016.11.034). (Also: e-print, arXiv:1506.03525v3 [math-ph], 2016; IHES preprint, M/15/15, 2015.)

[LapRaZu3] M. L. Lapidus, G. Radunović and D. Žubrinić, Complex dimensions of fractals and meromorphic extensions of fractal zeta functions, J. Math. Anal. Appl. No. 1, 453 (2017), 458-484. (doi.org/10.1016/j.jmaa.2017.03.059.) (Also: e-print, arXiv:1508.04784v3 [math-ph], 2016.)

[LapRaZu4] M. L. Lapidus, G. Radunović and D. Žubrinić, Zeta functions and complex dimensions of relative fractal drums: Theory, examples and applications, Dissertationes Mathematicae 526 (2017), 1-105. DOI: 10.4064/dm757-4-2017. (Also: e-print, arXiv:1603.00946v3 [math-ph], 2016.)

[LapRaZu5] M. L. Lapidus, G. Radunović and D. Žubrinić, Fractal tube formulas and a Minkowski measurability criterion for compact subsets of Euclidean spaces, Discrete and Continuous Dynamical Systems Ser. S No. 1, 12 (2019), 105-117. DOI: 10.3934/dcdss.2019007. (Also: e-print, arXiv:1411.5733v5 [math-ph], 2017; IHES preprint, IHES/M/15/17, 2015.)

[LapRaZu6] M. L. Lapidus, G. Radunović and D. Žubrinić, Fractal tube formulas for compact sets and relative fractal drums: Oscillations, complex dimensions and fractality, Journal of Fractal Geometry 5 (2018), 1-119. DOI: 10.4171/JFG/57. (Also: e-print, arXiv:1604.08014v5 [math-ph], 2018.)

[LapRaZu7] M. L. Lapidus, G. Radunović and D. Žubrinić, Minkowski measurability criteria for compact sets and relative fractal drums in Euclidean spaces, submitted for publication in the Proceedings of the 6th Cornell Conference on Analysis, Probability and Mathematical Physics on Fractals (Ithaca, June 2017), World Scientific, Singapore and London, 2019. (Also: e-print, arXiv:1609.04498v2 [math-ph], 2018.)

[LapRaZu8] M. L. Lapidus, G. Radunović and D. Žubrinić, Fractal zeta functions and complex dimensions of relative fractal drums, survey article, J. Fixed Point Theory Appl. No. 2, 15 (2014), 321-378. Festschrift issue in honor of Haim Brezis' 70th birthday. (DOI: 
10.1007/s11784-014-0207-y.) (Also: e-print, arXiv:1407.8094v3 [math-ph], 2014; IHES preprint, IHES/M/14, 2015.)

[LapRaZu9] M. L. Lapidus, G. Radunović and D. Žubrinić, Fractal zeta functions and complex dimensions: A general higher-dimensional theory, survey article, in: Fractal Geometry and Stochastics V (C. Bandt, K. Falconer and M. Zähle, eds.), Proc. Fifth Internat. Conf. (Tabarz, Germany, March 2014), Progress in Probability, vol. 70, Birkhäuser/Springer Internat., Basel, Boston and Berlin, 2015, pp. 229-257; DOI: 10.1007/978-3-319-18660-3_13. (Based on a plenary lecture given by the first author at that conference.) (Also: e-print, arXiv: 1502.00878v3 [math.CV], 2015; IHES preprint, IHES/M/15/16, 2015.)

[LapRaZu10] M. L. Lapidus, G. Radunović and D. Žubrinić, Fractal zeta functions and logarithmic gauge Minkowski measurability (tentative title), in preparation, 2018.

[LapRo] M. L. Lapidus and J. A. Rock, Towards zeta functions and complex dimensions of multifractals, Complex Variables and Elliptic Equations No. 6, 54 (2009), 545-560. (Also: e-print, arXiv: math-ph/0810.0789, 2008.)

[LapRoZu] M. L. Lapidus, J. A. Rock and D. Žubrinić, Box-counting fractal strings, zeta functions, and equivalent forms of Minkowski dimension, in: Fractal Geometry and Dynamical Systems in Pure and Applied Mathematics I: Fractals in Pure Mathematics (D. Carfi, M. L. Lapidus, E. P. J. Pearse and M. van Frankenhuijsen, eds.), Contemporary Mathematics, vol. 600, Amer. Math. Soc., Providence, R. I., 2013, pp. 239-271. (Also: e-print, arXiv:1207.6681v3 [math-ph], 2013; IHES preprint, IHES/M/12/22, 2012.)

[LapSar] M. L. Lapidus and J. J. Sarhad, Dirac operators and geodesic metric on the harmonic Sierpinski gasket and other fractal sets, Journal of Noncommutative Geometry No. 4, 8 (2014), 947-985. (DOI: 10.4171/JNCG/174.) (Also: e-print, arXiv:1212.0878v3 [math.MG], 2015; IHES preprint, IHES/M/12/32, 2012.)

[Lap-vF1] M. L. Lapidus and M. van Frankenhuijsen, Complex dimensions of fractal strings and oscillatory phenomena in fractal geometry and arithmetic, in: Spectral Problems in Geometry and Arithmetic (T. Branson, ed.), Contemporary Mathematics, vol. 237, Amer. Math. Soc., Providence, R. I., 1999, pp. 87-105.

[Lap-vF2] M. L. Lapidus and M. van Frankenhuijsen, Fractal Geometry and Number Theory: Complex Dimensions of Fractal Strings and Zeros of Zeta Functions, Birkhäuser, Boston, 2000 .

[Lap-vF3] M. L. Lapidus and M. van Frankenhuijsen, Fractal Geometry, Complex Dimensions and Zeta Functions: Geometry and Spectra of Fractal Strings, Springer Monographs in Mathematics, Springer, New York, 2006.

[Lap-vF4] M. L. Lapidus and M. van Frankenhuijsen, Fractal Geometry, Complex Dimensions and Zeta Functions: Geometry and Spectra of Fractal Strings, second revised and enlarged edition (of the 2006 edn., Lap-vF3), Springer Monographs in Mathematics, Springer, New York, 2013.

[Lap-vF5] M. L. Lapidus and M. van Frankenhuijsen, Complex dimensions of self-similar fractal strings and Diophantine approximation, J. Experimental Math., No. 1, 12 (2003), 4169. (Also: MSRI Preprint No. 2001-040, Mathematical Sciences Research Institute (MSRI), Berkeley, November 2001.)

[Lap-vF6] M. L. Lapidus and M. van Frankenhuijsen (eds.), Fractal Geometry and Applications: A Jubilee of Benoit Mandelbrot, Proc. Symp. Pure Math., vol. 72, Parts 1 \& 2, Amer. Math Soc., Providence, R. I., 2004. (Part 1: Analysis, Number Theory, and Dynamical Systems. Part 2: Multifractals, Probability and Statistical Mechanics, Applications.)

[Lap-vF7] M. L. Lapidus and M. van Frankenhuijsen, Fractality, self-similarity and complex dimensions, in Lap-vF6. Part 1, pp. 349-372].

[LapWat] M. L. Lapidus and S. Watson, Ahlfors metric measure spaces, fractal zeta functions and complex dimensions (tentative title), in preparation, 2017.

[Lau] A. Laurincikas, Limit Theorems for the Riemann Zeta-Function, Kluwer Academic Publishers, Dordrecht, 1996.

[LeviVa] M. Levitin and D. Vassiliev, Spectral asymptotics, renewal theorem, and the Berry conjecture for a class of fractals, Proc. London Math. Soc. (3) 72 (1996), 188-214.

[LevyMen] J. Lévy-Véhel and F. Mendivil, Multifractal and higher-dimensional zeta functions, Nonlinearity, No. 1, 24 (2011), 259-276.

[LiRadz] X. Li and M. Radziwill, The Riemann zeta function on vertical arithmetic progressions, Internat. Math. Research Notices 2 (2015), 325-354. 
[Lids] V. B. Lidskii, Non-Selfadjoint operators with a trace, Dokl. Akad. Nauk. SSSR 125 (1959), 485-487.

[LopGar1] F. López García, A decomposition technique for integrable functions with applications to the divergence problem, J. Math. Anal. Appl. 418 (2014), 79-99.

[LopGar2] F. López García, Weighted Korn inequality on John domains, preprint, 2015.

[MacL] S. Mac Lane, Homology, Classics in Mathematics, Springer, New York, 1995. (Original edition: 1963.)

[Man] B. B. Mandelbrot, The Fractal Geometry of Nature, revised and enlarged edition (of the 1977 French edn.), W. H. Freeman, New York, 1983.

[Mani] Y. Manin, Lectures on zeta functions and motives (according to Deninger and Kurokawa), in: Columbia University Number Theory Seminar (New York, 1992). Astérisque 228 (1995), pp. 121-163.

[ManFra] B. B. Mandelbrot and M. Frame, A primer of negative test dimensions and degrees of emptiness of latent sets, Fractals No. 1, 17 (2009), 1-14.

[MartVuo] O. Martio and M. Vuorinen, Whitney cubes, $p$-capacity, and Minkowski content, Exposition Math. No. 1, 5 (1987), 17-40.

[Mat] P. Mattila, Geometry of Sets and Measures in Euclidean Spaces: Fractals and Rectifiability, Cambridge Univ. Press, Cambridge, 1995.

[Maz] V. G. Maz'ja, Sobolev Spaces, Springer-Verlag, Berlin, 1985.

[McMul] C. T. McMullen, Kleinian groups and John domains, Topology No. 3, 7 (1998), 485-496.

[Mel1] R. B. Melrose, Weyl's conjecture for manifolds with concave boundary, in: Geometry of the Laplace Operator, Proc. Sympos. Pure Math, vol. 36, Amer. Math. Soc., Providence, R. I., 1980, pp. 254-274.

[Mel2] R. B. Melrose, The trace of the wave group, Contemporary Mathematics, vol. 27, Amer. Math. Soc., Providence, R. I., 1984, pp. 127-167.

[Met1] G. Métivier, Etude asymtotique des valeurs propres et de la fonction spectrale de problèmes aux limites, Thèse de Doctorat d'Etat, Mathématiques, Université de Nice, France, 1976.

[Met2] G. Métivier, Valeurs propres de problèmes aux limites elliptiques irréguliers, Bull. Soc. Math. France Mém. 51-52 (1977), 125-219.

[Mink] H. Minkowski, Theorie der konvexen Körper, insbesondere Begründung ihres Oberflächenbegriffs, in: Gesammelte Abhandlungen von Hermann Minkowski (part II, Chapter XXV), Chelsea, New York, 1967, pp. 131-229. (Originally reprinted in: Gesamm. Abh., vol. II, Leipzig, 1911.)

[MolVai] S. Molchanov and B. Vainberg, On spectral asymptotics for domains with fractal boundaries, Commun. Math. Phys. 183 (1997), 85-117.

[MorSep] G. Mora and J.M. Sepulcre, Privileged regions in critical strips of non-lattice Dirichlet polynomials, Complex Anal. Oper. Theory No. 4, 7 (2013), 1417-1426.

[MorSepVi1] G. Mora, J. M. Sepulcre and T. Vidal, On the existence of exponential polynomials with prefixed gaps, Bull. London Math. Soc. No. 6, 45 (2013), 1148-1162.

[MorSepVi2] G. Mora, J. M. Sepulcre and T. Vidal, On the existence of fractal strings whose set of dimensions of fractality is not perfect, Rev. R. Acad. Cienc. Exactas Fís. Nat. Ser. A Math. RACSAM No. 1, 109 (2015), 11-14.

[Mora] P. A. P. Moran, Additive functions of intervals and Hausdorff measure, Math. Proc. Cambridge Philos. Soc. 42 (1946) 15-23.

[Murt1] M. Ram Murty, A motivated introduction to the Langlands program, in: Advances in Number Theory (F. Gouvêa and N. Yui, eds.), Oxford Science Publications, Oxford Univ. Press, Oxford, 1993, pp. 37-66.

[Murt2] M. Ram Murty, Selberg's conjectures and Artin's L-functions, Bull. Amer. Math. Soc. (N. S.) 31 (1994), 1-14.

[Murt3] M. Ram Murty, Selberg's conjectures and Artin L-functions, II, in: Current Trends in Mathematics and Physics (S. D. Adhikari, ed.), Narosa, New Delhi, 1995, pp. 154-168.

[O11] L. Olsen, Multifractal tubes: Multifractal zeta functions, multifractal Steiner tube formulas and explicit formulas, in: Fractal Geometry and Dynamical Systems in Pure and Applied Mathematics I: Fractals in Pure Mathematics (D. Carfi, M. L. Lapidus and M. van Frankenhuijsen, eds.), Contemporary Mathematics, vol. 600, Amer. Math. Soc., Providence, R. I., 2013, pp. 291-326. (dx.doi.org/10.1090/conm/600/11920.)

[O12] L. Olsen, Multifractal tubes, in: Further Developments in Fractals and Related Fields, Trends in Mathematics, Birkhäuser/Springer, New York, 2013, pp. 161-191. 
[Oort] F. Oort, The Weil conjectures. Nw. Archief v. Wiskunde 5 Ser. 15, No. 3, (2014) 211-219.

[Ove] M. Overholt, A Course in Analytic Number Theory, Graduate Studies in Mathematics, vol. 160, Amer. Math. Soc., Providence, R. I., 2014.

[Oxl] J. G. Oxley, Matroid Theory, Oxford Graduate Texts in Mathematics, Oxford Univ. Press, Oxford, 2006.

[ParrPol1] W. Parry and M. Pollicott, An analogue of the prime number theorem and closed orbits of Axiom A flows, Annals of Math. 118 (1983), 573-591.

[ParrPol2] W. Parry and M. Pollicott, Zeta Functions and the Periodic Orbit Structure of Hyperbolic Dynamics, Astérisque, vols. 187-188, Soc. Math. France, Paris, 1990.

[ParsSh1] A. N. Parshin and I. R. Shafarevich (eds.), Number Theory, vol. I, Introduction to Number Theory, Encyclopedia of Mathematical Sciences, vol. 49, Springer-Verlag, Berlin, 1995. (Written by Yu. I. Manin and A. A. Panchishkin.)

[ParsSh2] A. N. Parshin and I. R. Shafarevich (eds.), Number Theory, vol. II. Algebraic Number Fields, Encyclopedia of Mathematical Sciences, vol. 62, Springer-Verlag, Berlin, 1992. (Written by H. Koch.)

[Pat] S. J. Patterson, An Introduction to the Theory of the Riemann Zeta-Function, Cambridge Univ. Press, Cambridge, 1988.

[Pe] E. P. J. Pearse, Canonical self-affine tilings by iterated function systems, Indiana Univ. Math. J. No. 6, 56 (2007), 3151-3169. (Also: e-print, arXiv:math.MG/0606111, 2006.)

[PeWi] E. P. J. Pearse and S. Winter, Geometry of canonical self-similar tilings, Rocky Mountain J. Math. No. 4, 42 (2012), 1327-1357. (Also: e-print: arXiv:0811.2187, 2009.)

$[\mathrm{Ph}]$ Pham The Lai, Meilleures estimations asymptotiques des restes de la fonction spectrale et des valeurs propres relatifs au laplacian, Math. Scand. 48 (1981), 5-38.

[Pit] H. R. Pitt, Tauberian Theorems, Oxford Univ. Press, London, 1958.

[PitWie] H. R. Pitt and N. Wiener, A generalization of Ikehara's theorem, J. Math. and Phys. M.I.T. 17 (1939), 247-258.

[Plem] J. Plemelj, Zur theorie Fredholmschen funktionalgleichung, Monatsh. Math. Phys. 15 (1904), 93-128.

[Poin1] H. Poincaré, Analysis situs, Journal de l'École Polytechnique (2) 1 (1895), 1-123.

[Poin2] H. Poincaré, Complément à L'Analysis Situs, Rendiconti del Circolo Matermatico di Palermo 13 (1899), 285-343.

[Poin3] H. Poincaré, Second complément à l'Analysis Situs, Proc. London Math. Soc. 32 (1900), 277-308.

[Poin4] H. Poincaré, Papers on Topology: Analysis Situs and Its Five Supplements, available online. (English translation by John Stillwell.)

[Poin5] H. Poincaré, Remarques diverses sur l'équation de Fredholm, Acta Math. 33 (1909), 57-86.

[Pomm] Ch. Pommerenke, Boundary Behavior of Conformal Maps, Springer, New York, 1992.

[Pos] A. G. Postnikov, Tauberian Theory and its Applications, Proc. Steklov Institute of Mathematics, vol. 144, 1979 (English translation, issue 2, 1980), Amer. Math. Soc., Providence, R. I., 1980.

[Put1] C. R. Putnam, On the non-periodicity of the zeros of the Riemann zeta-function, Amer. J. Math. 76 (1954), 97-99.

[Put2] C. R. Putnam, Remarks on periodic sequences and the Riemann zeta-function, Amer. J. Math. 76 (1954), 828-830.

[Ra1] G. Radunović, Fractal Analysis of Unbounded Sets in Euclidean Spaces and Lapidus Zeta Functions, Ph. D. Thesis, University of Zagreb, Zagreb, Croatia, 2015.

[Ra2] G. Radunović, Fractality and Lapidus zeta functions at infinity, Mathematical Communications 21 (2016), 141-162. (Also, e-print, arXiv:1510.06449v2 [math-ph], 2016.)

[Ram] R. Rammal, Spectrum of harmonic excitations on fractals, J. de Physique 45 (1984), 191206.

[RamTo] R. Rammal and G. Toulouse, Random walks on fractal structures and percolation cluster, J. Physique Lettres 44 (1983), L13-L22.

[RatWi] J. Rataj and S. Winter, Characterization of Minkowski measurability in terms of surface area, J. Math Anal. Appl. 400 (2013), 120-132.

[ReSi1] M. Reed and B. Simon, Methods of Modern Mathematical Physics, vol. I, Functional Analysis, revised and enlarged edition (of the 1975 edn.), Academic Press, New York, 1980.

[ReSi2] M. Reed and B. Simon, Methods of Modern Mathematical Physics, vol. II, Fourier Analysis, Self-Adjointness, Academic Press, New York, 1975. 
[ReSi3] M. Reed and B. Simon, Methods of Modern Mathematical Physics, vol. IV, Analysis of Operators, Academic Press, New York, 1979.

[Rei1] A. Reich, Universelle Werterverteilung von Eulerprodukten, Nachr. Akad. Wiss. Göttingen Math.-Phys. KI. II (1977), no. 1, 1-17.

[Rei2] A. Reich, Wertevereteilung von Zetafunktionen, Arch. Math. 34 (1980), 440-451.

[Res] M. Resman, Invariance of the normalized Minkowski content with respect to the ambient space, Chaos, Solitons \& Fractals 57 (2013), 123-128. (Also: e-print, arXiv:1207.3279v1, 2012.)

[Rie] B. Riemann, Ueber die Anzahl der Primzahlen unter einer gegebenen Grösse, Monatsb. der Berliner Akad. 1858/60, pp. 671-680. (English translation in [Edw Appendix, pp. 229-305].)

[Ru1] W. Rudin, Real and Complex Analysis, third edition, McGraw-Hill, New York, 1987.

[Ru2] W. Rudin, Functional Analysis, second edition (of the 1973 edn.), McGraw-Hill, New York, 1991.

[Rue1] D. Ruelle, Generalized zeta-functions for Axiom A basic sets, Bull. Amer. Math. Soc. 82 (1976), 153-156.

[Rue2] D. Ruelle, Zeta functions for expanding maps and Anosov flows, Invent. Math. 34 (1978), 231-242.

[Rue3] D. Ruelle, Thermodynamic Formalism, Addison-Wesley, Reading, 1978.

[Rue4] D. Ruelle, Dynamical Zeta Functions for Piecewise Monotone Maps of the Interval, CRM Monographs Ser. (Centre de Recherches Mathématiques, Université de Montréal), vol. 4, Amer. Math. Soc., Providence, R. I., 1994.

[Sab1] C. Sabot, Integrated density of states of self-similar Sturm-Liouville operators and holomorphic dynamics in higher dimension, Ann. Inst. H. Poincaré Probab. Statist. 37 (2001), $275-311$.

[Sab2] C. Sabot, Spectral properties of self-similar lattices and iteration of rational maps, Mémoires Soc. Math. France (New Series) No. 92 (2003), 1-104.

[Sab3] C. Sabot, Spectral analysis of a self-similar Sturm-Liouville operator, Indiana Univ. Math. J. 54 (2005), 645-668.

[Sarn] P. Sarnak, L-functions, in: Proc. Internat. Congress Math., Berlin, 1988 (G. Fischer and U. Rehmann, eds.), vol. I, pp. 453-465. Documenta Math. J. DMV (Extra Volume ICM 98), 1998.

[Sc] M. Schechter, Operator Methods in Quantum Mechanics, Dover Publications, 2003.

[Schl] W. Schlag, A Course in Complex Analysis and Riemann Surfaces, Graduate Studies in Mathematics, vol. 154, Amer. Math. Soc., Providence, R. I., 2014.

[Schm] F. K. Schmidt, Analytische Zahlentheorie in Körpern der Charakteristik p. Math. Zeitschrift 33 (1931), 1-32.

[Schn] R. Schneider, Convex Bodies: The Brunn-Minkowski Theory, Encyclopedia of Mathematics and its Applications, vol. 44, Cambridge Univ. Press, Cambridge, 2003. (Reprinted from the 1993 edition.)

[Schw] L. Schwartz, Théorie des Distributions, revised and enlarged edition (of the 1951 edn.), Hermann, Paris, 1996.

[See1] R. T. Seeley, Complex powers of elliptic operators, in: Proc. Sympos. Pure Math, vol. 10, Amer. Math. Soc., Providence, R. I., 1967, pp. 288-307.

[See2] R. T. Seeley, A sharp asymptotic remainder estimate for the eigenvalues of the Laplacian in a domain of $\mathbb{R}^{3}$, Adv. in Math. 29 (1978), 244-269.

[See3] R. T. Seeley, An estimate near the boundary for the spectral counting function of the Laplace operator, Amer. J. Math. 102 (1980), 869-902.

[Sel1] A. Selberg, Old and new conjectures and results about a class of Dirichlet series, in: Proc. Amalfi Conf. in Analytic Number Theory, Sept. 1989 (E. Bombieri, et al., eds.), Università di Salerno, 1992, pp. 367-385. (Reprinted in [Sel2 pp. 47-63].)

[Sel2] A. Selberg, Collected Papers, vol. II, Springer-Verlag, New York, 1991.

[Ser] J.-P. Serre, A Course in Arithmetic, English translation, Springer-Verlag, Berlin, 1973.

[Shi] T. Shima, On eigenvalue problems for Laplacians on p.c.f. self-similar sets, Japan J. Indust. Appl. Math. 13 (1996), 1-23.

[Shis] M. Shishikura, The Hausdorff dimension of the boundary of the Mandelbrot set and Julia sets, Ann. of Math. 147 (1998), 225-267.

[Sim1] B. Simon, Notes on infinite determinants of Hilbert space operators, Adv. in Math. 24 (1977), 244-273. 
[Sim2] B. Simon, Trace Ideals and Their Applications, second edition, Mathematical Surveys and Monographs, vol. 120, Amer. Math. Soc., Providence, R. I., 2005.

[Sim3] B. Simon, Operator Theory: A Comprehensive Course in Analysis, Part 4, Amer. Math. Soc., Providence, R. I., 2015.

[Smit] F. Smithies, The Fredholm theory of integral equations, Duke Math. J. 8 (1941), 107-130.

[Sta] L. L. Stachó, On the volume function of parallel sets, Acta Sci. Math. (Szeged), 38 (1976), $365-374$

[Stein] J. Steiner, Über parallele Flächen, Monatsb. preuss. Akad. Wiss., Berlin, 1840, pp. 114118. (Reprinted in: Gesamm. Werke, vol. II, pp. 173-176.)

[Steu] J. Steuding, Value-Distribution and L-Functions, Lecture Notes in Mathematics, vol. 1877, Springer, Berlin, 2007.

[Str] R. S. Strichartz, Differential Equations on Fractals: A Tutorial, Princeton Univ. Press, Princeton, 2006.

[Tep1] A. Teplyaev, Spectral zeta function of symmetric fractals, in: Fractal Geometry and Stochastics III, Progress in Probability, vol. 57, Birkhäuser-Verlag, Basel, 2004, pp. 245262.

[Tep2] A. Teplyaev, Spectral zeta functions of fractals and the complex dynamics of polynomials, Trans. Amer. Math. Soc. 359 (2007), 4339-4358. (Also: e-print, arXiv:math.SP/0505546, 2005.)

[Tha1] K. Thas (ed.), Absolute Arithmetic and $\mathbb{F}_{1}$-Geometry, European Math. Soc., Zürich, 2016.

[Tha2] K. Thas, The combinatorial motivic structure of $\mathbb{F}_{1}$-schemes, in Tha1 pp. 83-159].

[Tit] E. C. Titchmarsh, The Theory of the Riemann Zeta-Function, second edition (revised by D. R. Heath-Brown), Oxford Science Publications, Oxford Mathematical Monographs, Oxford Univ. Press, Oxford, 1986.

[Tri1] C. Tricot, Mesures et Dimensions, Thèse de Doctorat d'Etat Es Sciences Mathématiques, Université Paris-Sud, Orsay, France, 1983.

[Tri2] C. Tricot, Dimensions aux bords d'un ouvert, Ann. Sci. Math. Québec 11 (1987), 205-235.

[Tri3] C. Tricot, Curves and Fractal Dimension, Springer-Verlag, Berlin, 1995.

[Tri4] C. Tricot, General Hausdorff functions, and the notion of one-sided measure and dimension, Ark. Mat. 48 (2010), 149-176.

[vB-Gi] M. van den Berg and P. B. Gilkey, A comparison estimate for the heat equation with an application to the heat content of the s-adic von Koch snowflake, Bull. London Math. Soc. 30 (1998), 404-412.

[Vel-San] A. Vélez-Santiago, Global regularity for a class of quasi-linear local and nonlocal elliptic equations on extension domains, J. Funct. Anal. No. 1, 269 (2015), 1-46.

[vF1] M. van Frankenhuijsen, Arithmetic progressions of zeros of the Riemann zeta function, $J$. Number Theory No. 2, 115 (2005), 360-370.

[vF2] M. van Frankenhuijsen, Riemann zeros in arithmetic progression, in: Fractal Geometry and Dynamical Systems in Pure and Applied Mathematics I:Fractals in Pure Mathematics (D. Carfi, M. L. Lapidus, E. P. J. Pearse and M. van Frankenhuijsen, eds.), Contemporary Mathematics, vol. 600, Amer. Math. Soc., Providence, R. I., 2013, pp. 365-380.

[vM1] H. von Mangoldt, Auszug aus einer Arbeit unter dem Titel: Zu Riemann's Abhandlung 'Über die Anzahl der Primzahlen unter einer gegebenen Grösse', Sitzungsberichte preuss. Akad. Wiss., Berlin, 1894, pp. 883-896.

[vM2] H. von Mangoldt, Zu Riemann's Abhandlung 'Über die Anzahl der Primzahlen unter einer gegebenen Grösse', J. Reine Angew. Math. 114 (1895), 255-305.

[Vor] S. M. Voronin, Theorem on the 'universality' of the Riemann zeta-function, Izv. Akad. Nauk. SSSR, Ser. Matem. 39 (1975), 475-486 (Russian); Math. USSR Izv. 9 (1975), 443-445.

[Wat] S. Watson, Fractal Zeta Functions: Ahlfors Spaces and Beyond, Ph.D. Dissertation, University of California, Riverside, U.S.A., June 2017.

[Wei1] A. Weil, On the Riemann hypothesis in function-fields, Proc. Nat. Acad. Sci. U.S.A. 27 (1941), 345-347. (Reprinted in [Wei6 vol. I, pp. 277-279].)

[Wei2] A. Weil, Sur les courbes algébriques et les variétés qui s'en déduisent, Pub. Inst. Math. Strasbourg VII (1948), pp. 1-85. (Reprinted in: Courbes algébriques et variétés abéliennes, Hermann, Paris, 1971.)

[Wei3] A. Weil, Number of solutions of equations in finite fields, Bull. Amer. Math. Soc. 55 (1949), 497-508. (Reprinted in Wei6 vol. I, pp. 399-410].) 
[Wei4] A. Weil, Fonction zêta et distributions, Séminaire Bourbaki, 18ième année, 1965/66, no. 312, Juin 1966, pp. 1-9. (Reprinted in Wei6 vol. III, pp. 158-163].)

[Wei5] A. Weil, Sur les formules explicites de la théorie des nombres, Izv. Mat. Nauk (Ser. Mat.) 36 (1972), 3-18; English translation in: Math. USSR, Izv. 6 (1973), 1-17. (Reprinted in Wei6 vol. III, pp. 249-264].)

[Wei6] A. Weil, André Weil: Oeuvres Scientifiques (Collected Papers), vols. I, II and III, second edition (with corrected printing), Springer-Verlag, Berlin and New York, 1980.

[Wein] S. Weinberg, The Quantum Theory of Fields, vol. III, Supersymmetry, Cambridge Univ. Press, Cambridge, 2000.

[Wel] D. J. A. Welsh, Matroid Theory, Academic Press, 1976. (Reprinted by Dover Publ., Mineola, 2010.)

[Wey1] H. Weyl, Über die Abhängigkeit der Eigenschwingungen einer Membran von deren Begrenzung, J. Reine Angew. Math. 141 (1912), 1-11. (Reprinted in Wey4 vol. I, pp. 431-441].)

[Wey2] H. Weyl, Das asymptotische Verteilungsgesetz der Eigenwerte linearer partieller Differentialgleichungen, Math. Ann. 71 (1912), 441-479. (Reprinted in Wey4 vol. I, pp. 393-430].)

[Wey3] H. Weyl, On the volume of tubes, Amer. J. Math. 61 (1939), 461-472. (Reprinted in Wey4 vol. III, pp. 658-669].)

[Wey4] H. Weyl, Hermann Weyl: Gesammelte Abhandlungen (Collected Works), vols. I and III, Springer-Verlag, Berlin and New York, 1968.

[Wi] S. Winter, Curvature measures and fractals, Dissertationes Math. (Rozprawy Mat.) 453 (2008), 1-66.

[WiZä] S. Winter and M. Zähle, Fractal curvature measures of self-similar sets, Adv. Geom. 13 (2013), 229-244.

[Wils] K. G. Wilson, Renormalization group and critical phenomena, I \& II, Phys. Rev. B4 (1971), 3174-3183 \& 3184-3205.

[Yo] K. Yosida, Functional Analysis, Graduate Text in Mathematics, fifth edition, SpringerVerlag, Berlin and New York, 1978.

[Zä1] M. Zähle, Integral and current representation of Federer's curvature measures, Arch. Math. 46 (1986), 557-567.

[Zä2] M. Zähle, Curvatures and currents for unions of sets with positive reach, Geom. Dedicata 23 (1987), 155-171.

[Zä3] M. Zähle, Lipschitz-Killing curvatures of self-similar random fractals, Trans. Amer. Math. Soc. No. 5, 363 (2011), 2663-2684. (DOI: 10.1090/S0002-9947-2010-05198-0.)

[Zä4] M. Zähle, Curvature measures of fractal sets, survey article, in: Fractal Geometry and Dynamical Systems in Pure and Applied Mathematics I: Fractals in Pure Mathematics (D. Carfi, M. L. Lapidus and M. van Frankenhuijsen, eds.), Contemporary Mathematics, vol. 600, Amer. Math. Soc., Providence, R. I., 2013, pp. 381-399. (dx.doi.org/10.1090/conm/600/11953.)

[Zu1] D. Žubrinić, Minkowski content and singular integrals, Chaos, Solitons 85 Fractals No. 1, 17 (2003), 169-177.

[Zu2] D. Žubrinić, Analysis of Minkowski contents of fractal sets and applications, Real Anal. Exchange No. 2, 31 (2005/2006), 315-354.

Department of Mathematics, University of California, Riverside, CA 92521, USA

E-mail address: lapidus@math.ucr.edu 